\title{
TUMOR-DERIVED EXTRACELLULAR VESICLES FOR CANCER DISEASE MANAGEMENT
}

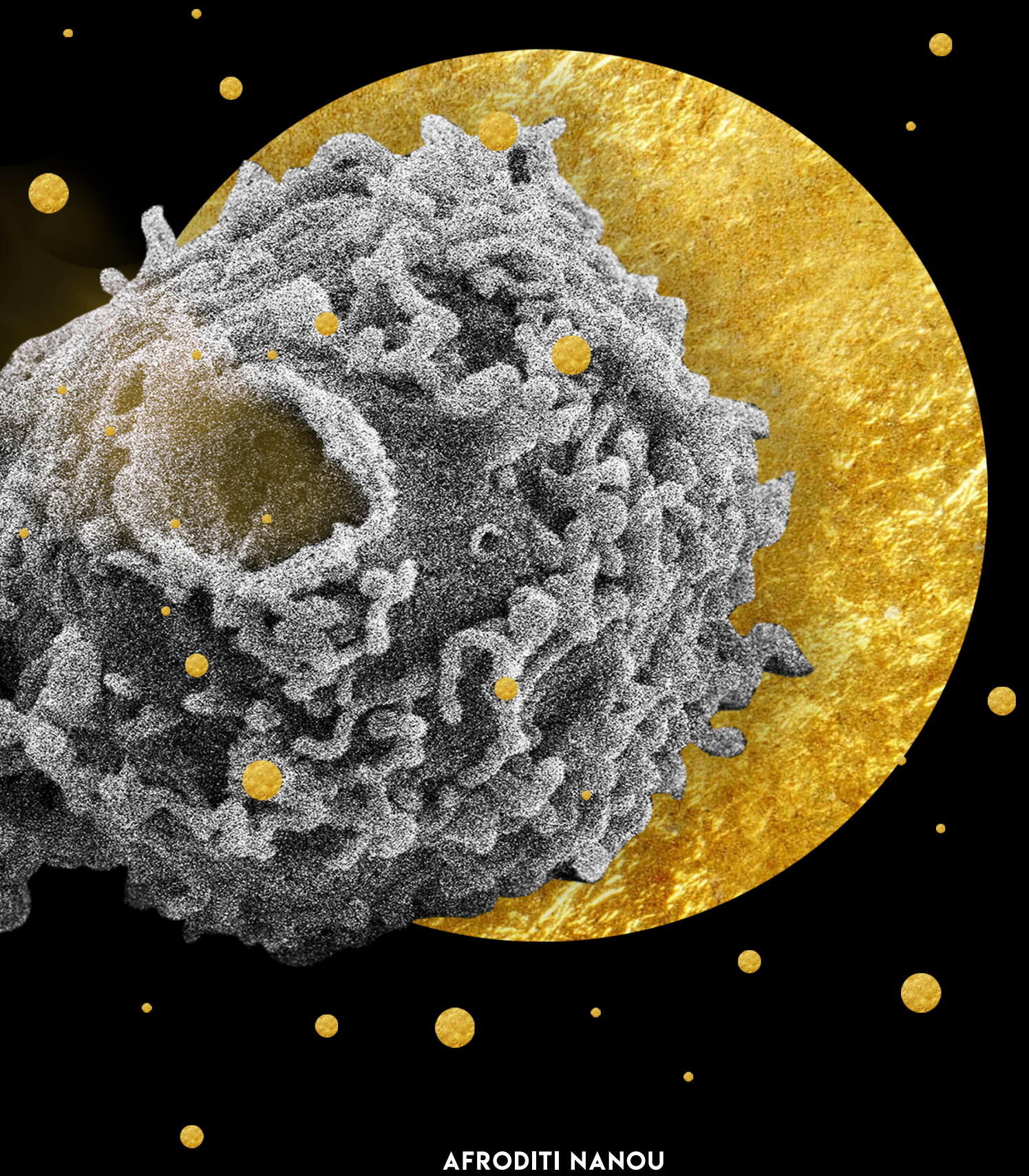




\section{TUMOR-DERIVED EXTRACELLULAR VESICLES FOR CANCER DISEASE MANAGEMENT}





\title{
TUMOR-DERIVED EXTRACELLULAR VESICLES FOR CANCER DISEASE MANAGEMENT
}

\author{
DISSERTATION
}

\author{
to obtain \\ the degree of doctor at the University of Twente, \\ on the authority of the rector magnificus, \\ Prof. dr. T.T.M. Palstra, \\ on account of the decision of the Doctorate Board, \\ to be publicly defended \\ on Thursday the 7th of May 2020 at 16.45 \\ by \\ Afroditi Nanou \\ born on the 16th of July 1987 \\ in Athens, Greece
}


This dissertation has been approved by:

Prof. dr. L.W.M.M. Terstappen, MD

Promotor

Cover design: $x M x$ Graphic Studio, www.xmxgraphics.com

Printed by: Proefschrift All in One (AIO), www.proefschrift-aio.nl

ISBN: 978-90-365-4985-1

DOI: $10.3990 / 1.9789036549851$

URL: https://doi.org/10.3990/1.9789036549851

(c) 2020 Afroditi Nanou, The Netherlands. All rights reserved. No parts of this thesis may be reproduced, stored in a retrieval system or transmitted in any form or by any means without permission of the author. Alle rechten voorbehouden. Niets uit deze uitgave mag worden vermenigvuldigd, in enige vorm of op enige wijze, zonder voorafgaande schriftelijke toestemming van de auteur. 


\section{Graduation Committee}

Chairman/Secretary:

Prof. dr. J.L. Herek

Supervisor:

Prof. dr. L.W.M.M. Terstappen, MD

Committee Members:

Prof. dr. J.S. De Bono, MD

Prof. dr. S. Sleijfer, MD

Prof. dr. H.J.M. Groen, MD

Prof. dr. ir. S. Le Gac

Prof. dr. J. Prakash
University of Twente

University of Twente

Institute of Cancer Research, UK

Royal Marsden, UK

Erasmus MC Cancer Institute

University Medical Center Groningen

University of Twente

University of Twente

\section{UNIVERSITY OF TWENTE. \\ Cancer - ID}

The research described in this thesis was conducted at the Medical Cell BioPhysics department of the University of Twente, Enschede, the Netherlands. This work was financially supported by the Netherlands Organisation for Scientific Research - Domain Applied and Engineering Sciences (NWO - TTW), under the Perspectief research program Cancer-ID, grant \#14190. 



\section{Table of contents}

Chapter 1

Chapter 2

Chapter 3

Chapter 4

Chapter 5

Chapter 6

Chapter 7

Chapter 8

Chapter 9

Chapter 10

Summary

List of publications

Acknowledgements

Curriculum Vitae
General introduction 9

Circulating Tumor Cells, tumor-derived Extracellular

Vesicles and plasma cytokeratins in castrationresistant prostate cancer patients

Tumor-derived extracellular vesicles in blood of metastatic cancer patients associate with overall survival

Leukocyte-derived Extracellular Vesicles in blood with and without EpCAM enrichment

Scanning Electron Microscopy of Circulating Tumor Cells and tumor-derived Extracellular Vesicles

HER2 expression on tumor-derived Extracellular Vesicles and Circulating Tumor Cells in metastatic breast cancer

Monitoring responses to therapy with EpCAM+ CTCs and large tdEVs

Endothelium- derived extracellular vesicles associate with poor prognosis in metastatic colorectal cancer Fusion of functional liposomes with cells: a versatile method for the supramolecular functionalization of cell membranes in vitro

Outlook 



\section{Chapter 1}

\section{General introduction}

Cancer remains one of the leading causes of morbidity and mortality worldwide ${ }^{1}$ with 17 million new cases and 9.6 million deaths ascribed to it, just in $2018^{2}$. Regardless these rather discouraging numbers, substantial advances in cancer treatment ${ }^{3}$, such as the introduction of immunotherapy ${ }^{4,5}$, have rendered some cancer cases to chronic diseases prolonging patients' survival and reducing the cancer death rate by $27 \%$ within the last 25 years (from 1991 to 2016 ) $^{1}$

The most important classification of cancer is malignant and benign with only the former one having the capability to invade and form metastases. Additional classification is based on the tissue from which cancer arises; carcinomas from epithelial tissues, sarcomas from connective and muscle tissue; gliomas from brain cells; leukaemias and lymphomas from hematopoietic cells. Carcinomas constitute the largest neoplasia class representing $80 \%$ of all cancers, including those of breast, colon, liver, lung and prostate and they are also the focus of the present thesis.

\subsection{Carcinogenesis}

Carcinogenesis is a complex process involving several steps ${ }^{6}$, namely the transformation of normal epithelial cells to immortalized ones, their development to hyperplasia, dysplasia, carcinoma in situ, invasive carcinoma (as shown in Figure $1.1^{7}$ ) and eventually the formation of secondary tumors.

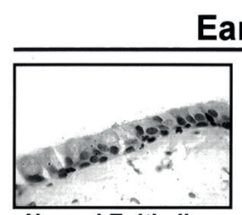

Normal Epithelium
Early

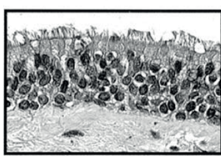

Hyperplasia

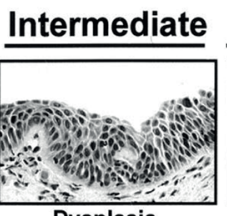

Dysplasia
Late

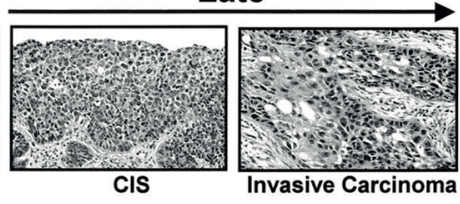

Figure 1.1. Morphological changes of epithelial tissue (in this case lung) during carcinogenesis. Modified from Hirsch et $\mathrm{al}^{7}$.

The transformation of cells to immortalized ones is the result of accumulated alterations at the genome or/and epigenome of the cell (eg activation of oncogenes and deactivation of tumor suppressor genes) and less frequently (less than $20 \%$ of the cases) is induced by oncoviruses. The genetic and epigenetic alterations are mainly 


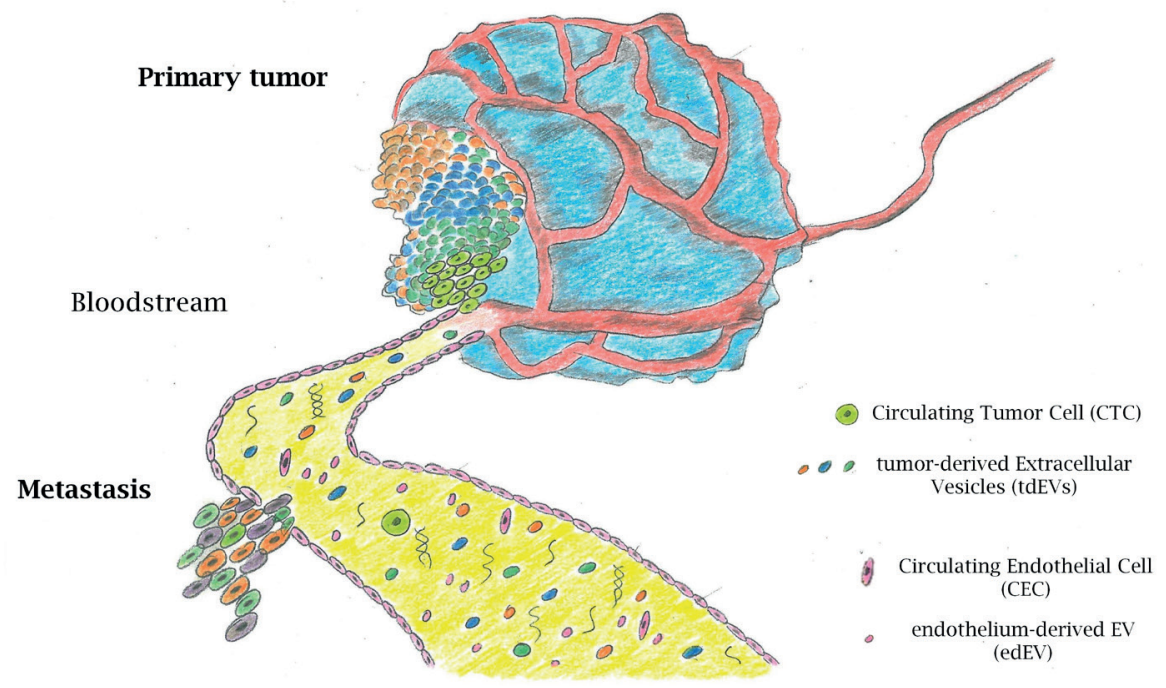

Figure 1.2. Illustration (by Eleni Hilari Nanou) of invasive carcinoma and formation of a secondary tumor (metastasis).

driven by threatening factors for cell viability and can assure prolonged survival and growth of cells that would normally undergo apoptosis or necrosis. In that sense, carcinogenesis could be considered as a desperate survival effort of cells that leads to their overgrowth at the expense of other cell populations disturbing cell balance and subsequently, tissue and organ functions.

The molecular events occurring in the transformed cancerous cells have been described extensively by Hanahan and Weinberg and are known as the "hallmarks of cancer"8,9. Apart from their uncontrollable cell proliferation and insensitivity to antigrowth signals, cancer cells promote angiogenesis. In addition, they have genome instability resulting in temporal and spatial heterogeneity, they are able to avoid immune surveillance, they have reprogrammed metabolic energetics and they acquire enhanced invasive capability forming local and distant metastases. This last hallmark is the main mortality cause counting for $90 \%$ of the lethal rates observed in cancer patients, even if metastasis itself is, paradoxically, very inefficient with less than $0.02 \%$ of disseminated cancer cells actually managing to form macroscopic tumors ${ }^{10,11}$

\subsection{Metastasis}

Metastasis is a multi-step process, whereby cancer cells detach -passively or activelyfrom the primary tumor, invade the blood circulation (intravasate), travel within the 


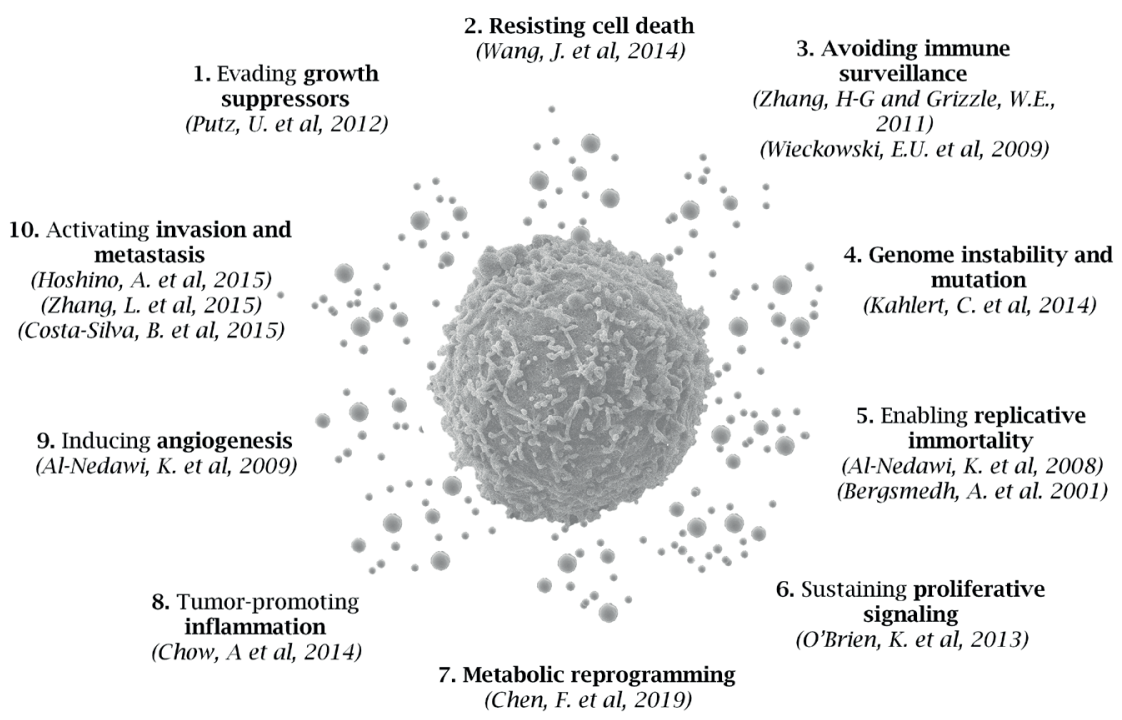

Figure 1.3. Promotion of tumor growth and invasion pleiotropically ${ }^{16-29}$ by (td)EVs via all different hallmarks of cancer.

bloodstream, exit the blood (extravasate) and colonize to a new site (formation of secondary tumor) ${ }^{12,13}$. These migrating cells, the so-called circulating tumor cells (CTCs), were firstly observed by Thomas Ashworth in $1869^{14}$ and are considered the initiators of metastasis ${ }^{15}$ (Figure 1.2).

Apart from the released CTCs, increasing body of evidence support that the tumor secretome (cytokines, hormones, growth factors, other proteins, nucleic acids and extracellular vesicles) contribute in converting a distant site to a "fertile" premetastatic niche ("soil") for the CTCs ("seeds") engaging various cell populations with diverse functions. More specifically, tumor-derived extracellular vesicles (with a size range between $30 \mathrm{~nm}$ and $10 \mu \mathrm{m}$ ) have emerged to an important player in intercellular communication, since they bear active molecular cargo (proteins, DNAs, mRNAs, microRNAs) encapsulated within their bi-phospholipid membrane with similar biochemical composition as the membrane of their cells of origin. Such a formation ensures the prolonged circulation of tdEVs in the blood circulation and the protection of their molecular cargo from degradation. Functional studies have demonstrated the pleiotropic roles of (td)EVs in enhancing each of the different aspects/hallmarks of cancer ${ }^{16-29}$, as summarized in Figure 1.3. Eventually, a whole network of cells is tightly interconnected and synchronized in response to signals in facilitating tumor progression and metastasis in a similar way as different musicians execute their unique transcript leading to a perfect orchestration. 


\subsection{Cancer diagnosis and treatment}

After the detection of a suspicious mass, a representative sample is collected for further examination (biopsy). In case pathologic evaluation reveals the presence of cancer, various tests can be performed to determine the extent of the disease and establish a treatment plan. For some cancers, neoadjuvant therapy is administrated to reduce the tumor burden and increase the likelihood of successful surgical removal of the tumor. After staging of the cancer, adjuvant therapy can be administrated for the cases with relatively high risk of recurrence. Accurate assessment of whether or not the cancer has spread beyond the primary tumor is of utmost importance. At the time of the doctor's assessment, only few patients will be diagnosed with metastatic disease. The appearance of metastatic disease in the rest of the patients can range from weeks to years implying that current determination of the stage of the disease is not adequate. Most staging methods are based on assessing a risk profile rather than detecting the minimal residual disease after primary intervention. The second approach is needed to discriminate patients with and without residual cancer and provide or prevent further treatment accordingly. Current imaging techniques do not have sufficient resolution and specificity to detect small lesions/micrometastases in the body. One avenue would be to detect the cancer cells in the blood before the formation of metastases ${ }^{30}$.

Once metastasis is diagnosed, a large arsenal of drugs is readily available for administration. Although many of the new drugs are effective, drug tolerance and resistance emerges in subsets of patients during the course of therapy. Identification of patients that will benefit from a drug as well as early recognition of patients with emerging drug resistance will greatly advance the effective management of metastatic cancer disease. Availability of the cancer cells of the patients during the course of treatment is needed to determine the optimal treatment plan. Taking biopsies from all metastatic sites to get a "complete snapshot" of the disease is however not feasible and increasing efforts are being made to obtain such information through a "real-time liquid biopsy".

\subsection{Liquid biopsies}

Many studies have investigated the potential of a liquid biopsy (mostly peripheral blood) to guide personalized treatments. The rationale behind is very simple: the formation of metastases rise from tumor-secreted "seeds" (CTCs or/and tdEVs) that reach the distant site by the lymphatic and blood circulatory system and lead to the formation of tumor cell colonies, as previously described (Figure 1.2). Therefore, the collection of a blood sample will contain tumor material that is more clinically relevant and informative than the solid biopsy of the primary tumor, as the circulating tumor material constitutes the primary player in the initiation of metastases. 


\subsubsection{Circulating Tumor Cells (CTCs)}

The isolation and profiling of circulating tumor cells (CTCs) from peripheral blood of cancer patients has been the most intensely investigated liquid biopsy. Although various techniques have been developed with different underlying working principles, only one has been FDA-cleared, namely the CellSearch system that immunomagnetically enriches CTCs from blood based on the expression of the Epithelial Cell Adhesion Molecule (EpCAM) on their membrane ${ }^{31,32 .}$

Importantly, the findings of several studies support the outperformance of Circulating Tumor Cells (CTCs) over computed tomography (CT) scans and prostate specific antigen (PSA) tests in terms of prognostic power as well as treatment response $\mathrm{e}^{33,34}$. The clinical utility of CTCs to predict benefit of patients to treatments has been (IMMC-38 $8^{34}$, SWOG S0500 ${ }^{35}$, COU-AA-30136, SWOG SO42137) or is being (STIC CTC study (ClinicalTrials.gov Identifier: NCT01710605) (38 $^{38}$ CTC-STOP (ClinicalTrials.gov Identifier: NCT03327662)) investigated in clinical trials. From a technical standpoint, the main challenge in the study of CTCs is their low frequency with patients with relatively good prognosis having no detectable CTCs in a tube of blood. Extrapolation of the CTC frequency to larger blood volumes showed that by analysis of 1 liter of blood, CTCs could be detected in all metastatic patients, and thus used as probes for optimal treatment monitoring ${ }^{39}$. The challenge of analyzing larger blood volumes can be addressed through the use of diagnostic leukaphaeresis (DLA) ${ }^{40,41}$.

\subsection{2 tumor-derived Extracellular Vesicles (tdEVs)}

Alternative or/and supplementary to CTCs is the "capture" and "interrogation" of tdEVs that have several biological functions in promoting cancer progression and metastasis, as briefly described previously (Figure 1.3). The proteomic and biochemical composition of the membrane and cargo of tdEVs reflects the cells of their origin. Furthermore, tdEVs are present in much higher frequencies than CTCs; hence, they hold promising potential to better reflect the tumor and provide us with a more complete image of its phenotypic and genetic heterogeneity. These tdEVs have a wide size range $(30 \mathrm{~nm}-10 \mu \mathrm{m})$ and can be classified into exosomes if they have endoplasmatic origin, microvesicles if they bud off the plasma cell membrane and apoptotic bodies if they are by-products of cell apoptosis, as graphically illustrated in Figure 1.4.

\subsection{NWO perspectief program Cancer-ID}

F.Coumans et al. firstly demonstrated that large $(>1 \mu \mathrm{m}) \mathrm{EpCAM}+, \mathrm{CK}+, \mathrm{CD} 45$ - particles with or without nucleus, that had been co-isolated with CTCs by the CellSearch assay predict the clinical outcome in metastatic prostate cancer patients ${ }^{42}$. His findings paved the pathway to the Dutch "Cancer-ID" program, a multi-disciplinary collaboration among 8 universities and 21 companies to investigate the potential of 
existing techniques as well as newly developed ones to discriminate EVs from non-EV particles and reveal the main differences between EVs from model cancer cell lines and EVs of healthy cells of origin. The gained knowledge would be eventually exploited for the isolation, detection and characterization of the whole size spectrum of tdEVs from biofluids of cancer patients.

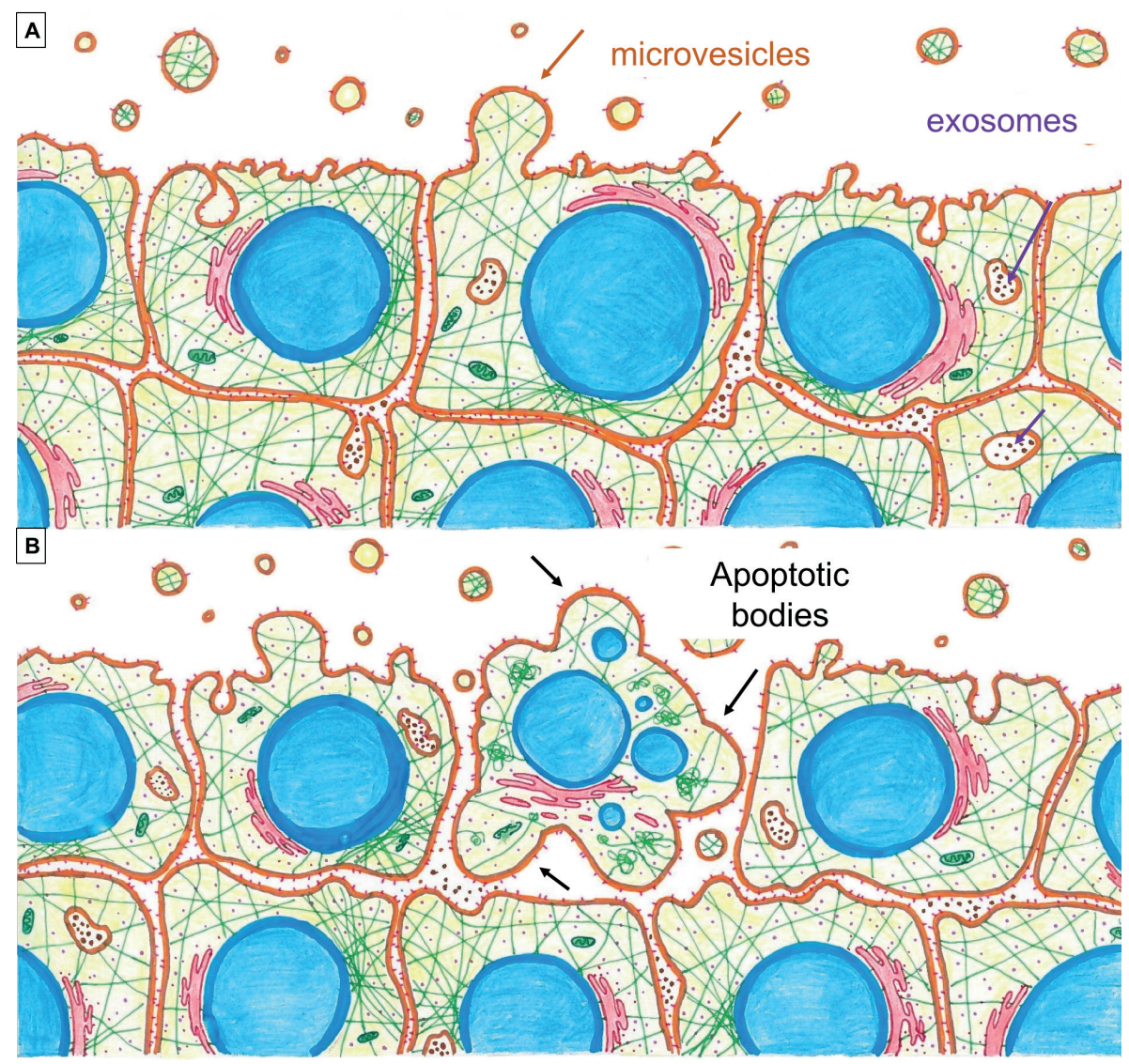

Figure 1.4. Schematic (by M. van Benthem) showing the active and passive release of Extracellular Vesicles (EVs) by epithelial cells over time $(A \rightarrow B)$. Cytoskeleton (cytokeratin 18) is indicated by a network of green lines, nucleus in blue, cytoplasm in yellow and plasma membrane in brown/orange. (A) Active secretion of EVs by healthy cells; (B) Nuclear fragmentation, membrane blebbing and caspase-cleaved cytokeratin 18 of a cell undergoing apoptosis.

Throughout the project, two main challenges regarding the detection of EVs in blood became apparent. To begin with, EV detection is hampered because EVs are outnumbered by the presence of non-EV particles in blood, like soluble proteins and lipoprotein particles at the low end of the EV size and density range, and platelets at the high end of the EV size and density range, as summarized in Figure $1.5^{43-45}$. Moreover, 
the concentration of larger lipoproteins, such as chylomicrons, depends on food intake, thereby emphasizing the need to discriminate EVs from other particles. To illustrate this challenge, we determined that $1 \mathrm{~mL}$ of human blood of metastatic castrationresistant prostate cancer patients contains about 10 large $(>1 \mu \mathrm{m})$ tdEVs ${ }^{46,47}$ and we extrapolated this to encompass the small tdEVs to arrive at an estimated $10^{4} \mathrm{tdEVs}$ per 1 $\mathrm{ml}$. Furthermore, the blood contains up to ${ }^{10^{16}}$ lipoproteins, up to $10^{9}$ platelets, and up to $10^{11}$ other EVs ${ }^{43,44,48-50}$, as shown in Figure 1.5. The second challenge is the heterogeneity of EVs in many aspects, including morphology ${ }^{51}$, $\operatorname{size}^{51-53}$, membrane composition ${ }^{47,53-57}$, and refractive index ${ }^{58,59}$, which complicates $\mathrm{EV}$ isolation, detection, and enumeration. Most groups worldwide have focused on the smaller-sized EV populations and their biological functions as they are considered to be actively secreted by their cells of origin in contrast to the larger EVs. However, the Cancer-ID project was interested in all classes of tdEVs and the evaluation of the sensitivity and specificity of several techniques to isolate and characterize them, as they could provide insights on the status of the tumor and be used as a real-time liquid biopsy. Whereas small tdEVs $(<1 \mu \mathrm{m})$ are likely to outnumber their larger counterparts, thereby offering better statistical significance, their identification and quantification is more challenging.

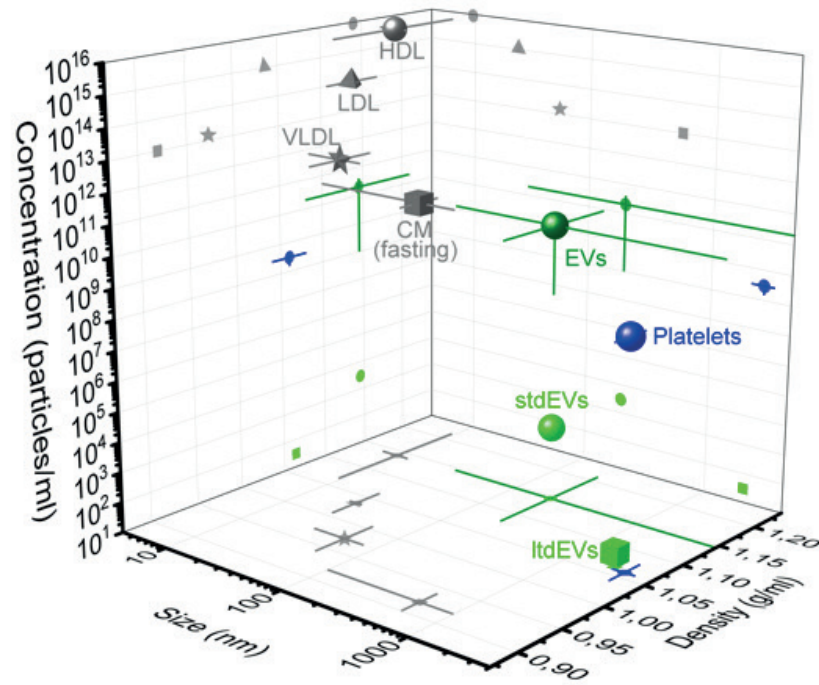

Figure 1.5. Concentration, size and density of plasma particles. 3D representation of concentration, size and density of extracellular vesicles (dark green circle), platelets (blue) and the High-Density Lipoproteins (HDL, grey circle), Low-density Lipoproteins (LDL, grey triangle), Very Low-Density Lipoproteins (VLDL, grey star) and Chylomicrons (CM, grey square) during fasting in blood. The average and standard deviation (lines) of the three parameters are indicated in the figure. Values are derived from literature $43,44,46,47,49,50$. The frequency of the large tumor derived extracellular vesicles (large tdEVs, light green circle) determined in the Cancer-ID program ${ }^{60}$ and the small tdEVs (small tdEVs, light green square) estimated after extrapolation using the frequency of large tdEVs. Adapted from Rikkert et $\mathrm{al}^{61}$. 


\subsection{Thesis outline}

In the present thesis, the clinical relevance of large EpCAM+ tumor-derived Extracellular Vesicles (tdEVs) was investigated. Clinical studies consisting of patient cohorts of different cancer types (metastatic breast, prostate, colorectal and non-small cell lung cancer) had been conducted to study the prognostic potential of Circulating Tumor Cells (CTCs) using the only FDA-cleared isolation technique for CTCs, the CellSearch system. Since the technique isolates CTCs based on their EPCAM expression on their surface (which is not expected to be found on blood cells), also large tdEVs with the same biomarker on their surface were co-isolated. The immunofluorescence images of all these studies were digitally stored. The availability of the open-source ACCEPT software that was developed in the frames of the PhD of L.L. Zeune ${ }^{62}$ allowed the enumeration of these tdEVs in an automated manner and the investigation of their prognostic utility and potential superiority to CTCs due to their presence in higher frequencies (Chapters 2 and 3).

To get some first insights whereas these tdEVs are a by-product of the Cellsearch procedure or they pre-exist in the blood samples, we checked the frequencies of leukocyte-derived EVs in blood samples of healthy individuals with and without EpCAM enrichment and found that their relative frequencies to leukocytes were very similar in both cases supporting our hypothesis that tdEVs pre-exist in blood samples (Chapter 4).

A protocol to allow Scanning Electron Microscopy of the isolated CTCs and tdEVs was developed and it was realized that not all objects isolated are detectable using the current staining included in the CTC kit used at the CellSearch system (Chapter 5).

The inclusion of an antibody recognizing the human epidermal growth factor receptor 2 (HER2), which is a therapeutic target in breast cancer revealed the presence of CKCTCs and tdEVs that were not reported so far when using the CellSearch system. Importantly, the majority of patients had all different subclasses of CTCs and tdEVs present and tdEVs could better reflect the HER2 status of the tumor due to their increased abundance compared to CTCs. That finding is very important in clinical practice since it could allow clinicians to decide more objectively on the treatment of their patients and follow the tumor phenotype in real-time by a simple blood test without the need of additional invasive biopsies (Chapter 6).

The use of tdEVs as a surrogate biomarker of treatment responses, similarly to previously reported CTCs, was investigated (Chapter 7). Our hypothesis was that the increased numbers of tdEVs in the blood circulation could lead to more confident conclusions regarding their true changes compared to CTCs. That would allow clinicians to better decide for continuation with the same line of treatment (in case of response) or a switch to a different treatment (in case of progression). 
The investigation of a different origin of extracellular vesicles, the so-called endothelium-derived extracellular vesicles (edEVs) showed that edEVs are indicators of prognosis in metastatic colorectal cancer patients (Chapter 8). Further research would reveal whereas they could potentially be used as markers to assess the response of patients to anti-angiogenic treatments. Importantly, their use could be expanded as diagnostic markers of individuals having an increased risk of cardiovascular diseases.

Last but not least, we were planning to use biotinylated liposomes to biotinylate the surface of EVs in plasma and isolate them using streptavidin columns. It soon became evident that there were 2 main limitations in regards to the biotinylation of EVs: 1 . The biotinylation was proportional to the surface of cells; hence, in case of EVs, it yielded much lower scores and 2. our existing flow cytometer (FACSaria II) was only able to evaluate the biotinylation of the larger EV population. However, we realized that the results were very useful to understand the uptake mechanism of liposomes by cells. The fusion of cells with liposomes implies the load of liposome cargo to the cells that can be extremely useful in case of efficient drug administration. Different liposome systems were compared and the results are reported in Chapter 9. 


\section{References}

1. Siegel RL, Miller KD, Jemal A. Cancer statistics, 2019. CA Cancer J Clin 2019; 69(1): 7-34; doi 10.3322/caac.21551.

2. UK CR. Worldwide cancer statistics. https://www.cancerresearchuk.org/health-professional/ cancer-statistics/worldwide-cancer - heading-Zero. Accessed January, 2020.

3. Pal SK, Miller MJ, Agarwal N, Chang SM, Chavez-MacGregor M, Cohen E et al. Clinical Cancer Advances 2019: Annual Report on Progress Against Cancer From the American Society of Clinical Oncology. Journal of clinical oncology : official journal of the American Society of Clinical Oncology 2019; 37(10): 834-849.

4. Pardoll DM. The blockade of immune checkpoints in cancer immunotherapy. Nature Reviews Cancer 2012; 12(4): 252-264.

5. Kantoff PW, Higano CS, Shore ND, Berger ER, Small EJ, Penson DF et al. Sipuleucel-T immunotherapy for castration-resistant prostate cancer. New Engl J Med 2010; 363(5): 411-422.

6. Briegel KJ. Embryonic transcription factors in human breast cancer. IUBMB life 2006; 58(3): 123-132.

7. Hirsch FR, Franklin WA, Gazdar AF, Bunn PA, Jr. Early detection of lung cancer: clinical perspectives of recent advances in biology and radiology. Clinical cancer research : an official journal of the American Association for Cancer Research 2001; 7(1): 5-22.

8. Hanahan D, Weinberg RA. The hallmarks of cancer. Cell 2000; 100(1): 57-70.

9. Hanahan D, Weinberg RA. Hallmarks of cancer: the next generation. Cell 2011; 144(5): 646-674.

10. Luzzi KJ, MacDonald IC, Schmidt EE, Kerkvliet N, Morris VL, Chambers AF et al. Multistep nature of metastatic inefficiency: dormancy of solitary cells after successful extravasation and limited survival of early micrometastases. The American journal of pathology 1998; 153(3): 865-873.

11. Christofori G. New signals from the invasive front. Nature 2006; 441(7092): 444-450.

12. Naba A, Clauser KR, Lamar JM, Carr SA, Hynes RO. Extracellular matrix signatures of human mammary carcinoma identify novel metastasis promoters. eLife 2014;3.

13. Guo W, Giancotti FG. Integrin signalling during tumour progression. Nat Rev Mol Cell Biol (10.1038/nrm1490) 2004; 5(10): 816-826.

14. Ashworth T. A case of cancer in which cells similar to those in the tumours were seen in the blood after death. Aust Med J 1869; 14: 146.

15. Chaffer CL, Weinberg RA. A perspective on cancer cell metastasis. Science 2011; 331(6024): 1559-1564; doi 10.1126/science.1203543.

16. Zhang H-G, Grizzle WE. Exosomes and cancer: a newly described pathway of immune suppression. Clinical Cancer Research 2011; 17(5): 959-964.

17. Wieckowski EU, Visus C, Szajnik M, Szczepanski MJ, Storkus WJ, Whiteside TL. Tumorderived microvesicles promote regulatory $\mathrm{T}$ cell expansion and induce apoptosis in tumorreactive activated CD8+ T lymphocytes. The Journal of Immunology 2009; 183(6):3720-3730.

18. Chen F, Chen J, Yang L, Liu J, Zhang X, Zhang Y et al. Extracellular vesicle-packaged HIF$1 \alpha$-stabilizing lncRNA from tumour-associated macrophages regulates aerobic glycolysis of breast cancer cells. Nature cell biology 2019; 21(4): 498-510. 
19. Chow A, ZhouW, Liu L, Fong MY, ChamperJ, Van Haute Detal. Macrophage immunomodulation by breast cancer-derived exosomes requires Toll-like receptor 2-mediated activation of NFxB. Sci Rep-Uk 2014; 4(1): 1-11.

20. Kahlert C, Melo SA, Protopopov A, Tang J, Seth S, Koch M et al. Identification of doublestranded genomic DNA spanning all chromosomes with mutated KRAS and p53 DNA in the serum exosomes of patients with pancreatic cancer. J Biol Chem 2014; 289(7): 3869-3875.

21. Al-Nedawi K, Meehan B, Micallef J, Lhotak V, May L, Guha A et al. Intercellular transfer of the oncogenic receptor EGFRvIII by microvesicles derived from tumour cells. Nature cell biology 2008; 10(5): 619-624.

22. Bergsmedh A, Szeles A, Henriksson M, Bratt A, Folkman MJ, Spetz A-L et al. Horizontal transfer of oncogenes by uptake of apoptotic bodies. Proceedings of the National Academy of Sciences 2001; 98(11): 6407-6411.

23. Al-Nedawi K, Meehan B, Kerbel RS, Allison AC, Rak J. Endothelial expression of autocrine VEGF upon the uptake of tumor-derived microvesicles containing oncogenic EGFR. Proceedings of the National Academy of Sciences 2009; 106(10): 3794-3799.

24. Wang J, Hendrix A, Hernot S, Lemaire M, De Bruyne E, Van Valckenborgh E et al. Bone marrow stromal cell-derived exosomes as communicators in drug resistance in multiple myeloma cells. Blood, The Journal of the American Society of Hematology 2014; 124(4): 555-566.

25. O'Brien K, Rani S, Corcoran C, Wallace R, Hughes L, Friel AM et al. Exosomes from triplenegative breast cancer cells can transfer phenotypic traits representing their cells of origin to secondary cells. European journal of cancer 2013; 49(8): 1845-1859.

26. Putz U, Howitt J, Doan A, Goh C-P, Low L-H, Silke J et al. The tumor suppressor PTEN is exported in exosomes and has phosphatase activity in recipient cells. Sci Signal 2012; 5(243): ra70-ra70.

27. Hoshino A, Costa-Silva B, Shen T-L, Rodrigues G, Hashimoto A, Mark MT et al. Tumour exosome integrins determine organotropic metastasis. Nature 2015; 527(7578): 329-335.

28. Zhang L, Zhang S, Yao J, Lowery FJ, Zhang Q, Huang W-C et al. Microenvironment-induced PTEN loss by exosomal microRNA primes brain metastasis outgrowth. Nature 2015; 527(7576): 100-104.

29. Costa-Silva B, Aiello NM, Ocean AJ, Singh S, Zhang H, Thakur BKetal. Pancreatic cancer exosomes initiate pre-metastatic niche formation in the liver. Nature cell biology 2015; 17(6): 816-826.

30. Coumans FA, Siesling S, Terstappen LW. Detection of cancer before distant metastasis. BMC Cancer 2013; 13: 283; doi 10.1186/1471-2407-13-283.

31. Coumans F, Terstappen L. Detection and Characterization of Circulating Tumor Cells by the CellSearch Approach. Methods in molecular biology (Clifton, NJ) 2015; 1347: 263-278.

32. Miller MC, Doyle GV, Terstappen LWMM. Significance of Circulating Tumor Cells Detected by the CellSearch System in Patients with Metastatic Breast Colorectal and Prostate Cancer. Journal of oncology 2010; 2010: 617421.

33. Budd GT, Cristofanilli M, Ellis MJ, Stopeck A, Borden E, Miller MC et al. Circulating tumor cells versus imaging - predicting overall survival in metastatic breast cancer. Clinical Cancer Research 2006; 12(21): 6403-6409. 
34. de Bono JS, Scher HI, Montgomery RB, Parker C, Miller MC, Tissing H et al. Circulating tumor cells predict survival benefit from treatment in metastatic castration-resistant prostate cancer. Clin Cancer Res 2008; 14(19): 6302-6309; doi 10.1158/1078-0432.CCR-08-0872.

35. Smerage JB, Barlow WE, Hortobagyi GN, Winer EP, Leyland-Jones B, Srkalovic G et al. Circulating tumor cells and response to chemotherapy in metastatic breast cancer: SWOG S0500. J Clin Oncol 2014; 32(31):3483-3489; doi 10.1200/JCO.2014.56.2561.

36. Scher HI, Heller G, Molina A, Attard G, Danila DC, Jia X et al. Circulating tumor cell biomarker panel as an individual-level surrogate for survival in metastatic castration-resistant prostate cancer. Journal of clinical oncology : official journal of the American Society of Clinical Oncology 2015; 33(12): 1348-1355.

37. Goldkorn A, Ely B, Quinn DI, Tangen CM, Fink LM, Xu T et al. Circulating tumor cell counts are prognostic of overall survival in SWOG SO421: a phase III trial of docetaxel with or without atrasentan for metastatic castration-resistant prostate cancer. Journal of clinical oncology : official journal of the American Society of Clinical Oncology 2014; 32(11): 1136-1142.

38. Bidard F-C, Jacot W, Dureau S, Brain E, Bachelot T, Bourgeois H et al. Abstract CTi4o: Circulating tumor cells as a tool to guide first line therapy in metastatic breast cancer: subgroup analyses of the STIC CTC Phase III utility trial. In: AACR; 2019.

39. Coumans FA, Ligthart ST, Uhr JW, Terstappen LW. Challenges in the enumeration and phenotyping of CTC. Clin Cancer Res 2012; 18(20): 5711-5718; doi 10.1158/1078-0432.CCR-12-1585.

40. Andree KC, Mentink A, Zeune LL, Terstappen LW, Stoecklein NH, Neves RP et al. Toward a real liquid biopsy in metastatic breast and prostate cancer: Diagnostic LeukApheresis increases CTC yields in a European prospective multicenter study (CTCTrap). International journal of cancer 2018; 143(10): 2584-2591.

41. Fischer JC, Niederacher D, Topp SA, Honisch E, Schumacher S, Schmitz N et al. Diagnostic leukapheresis enables reliable detection of circulating tumor cells of nonmetastatic cancer patients. Proc Natl Acad Sci U S A 2013; 110(41): 16580-16585; doi 10.1073/pnas.1313594110.

42. Coumans FAW, Doggen CJM, Attard G, de Bono JS, Terstappen LWMM. All circulating EpCAM $+C K+C D 45$-objects predict overall survival in castration-resistant prostate cancer. Ann Oncol 2010; 21(9): 1851-1857; doi 10.1093/annonc/mdq030.

43. Simonsen JB. What are we looking at? Extracellular vesicles, lipoproteins, or both? Circ Res 2017; 121(8): 920-922; e-pub ahead of print 2017/10/01; doi 10.1161/circresaha.117.311767.

44. Kuchinskiene Z, Carlson LA. Composition, concentration, and size of low density lipoproteins and of subfractions of very low density lipoproteins from serum of normal men and women. J Lipid Res 1982; 23(5): 762-769; e-pub ahead of print 1982/07/01.

45. Corash L, Costa JL, Shafer B, Donlon JA, Murphy D. Heterogeneity of human whole blood platelet subpopulations. III. Density-dependent differences in subcellular constituents. Blood 1984; 64(1): 185-193; e-pub ahead of print 1984/07/01.

46. Nanou A MM, Zeune LL, de Wit S, Punt CJA, Groen HJM, Hayes DF, de Bono JS, Terstappen LWMM. Tumour-derived extracellular vesicles in blood of metastatic cancer patients associate with overall survival. British Journal of Cancer (Article) 2020; doi 10.1038/s41416-019-0726-9. 
47. Nanou A, Coumans FAW, van Dalum G, Zeune LL, Dolling D, Onstenk W et al. Circulating tumor cells, tumor-derived extracellular vesicles and plasma cytokeratins in castrationresistant prostate cancer patients. Oncotarget 2018; 9(27): 19283-19293; e-pub ahead of print 2018/05/04; doi 10.18632/oncotarget.25019.

48. Ricklefs FL, Maire CL, Reimer R, Duhrsen L, Kolbe K, Holz M et al. Imaging flow cytometry facilitates multiparametric characterization of extracellular vesicles in malignant brain tumours. J Extracell Vesicles 2019; 8(1): 1588555; e-pub ahead of print 2019/04/06; doi 10.1080/20013078.2019.1588555.

49. Coumans F, van Dalum G, Terstappen L. CTC Technologies and Tools. Cytometry A 2018; 93(12): 1197-1201; doi 10.1002/cyto.a.23684.

50. Johnsen KB, Gudbergsson JM, Andresen TL, Simonsen JB. What is the blood concentration of extracellular vesicles? Implications for the use of extracellular vesicles as blood-borne biomarkers of cancer. Biochim Biophys Acta Rev Cancer 2019; 1871(1): 109-116; e-pub ahead of print 2018/12/12; doi 10.1016/j.bbcan.2018.11.006.

51. Rikkert LG, Nieuwland R, Terstappen L, Coumans FAW. Quality of extracellular vesicle images by transmission electron microscopy is operator and protocol dependent. J Extracell Vesicles 2019; 8(1): 1555419; e-pub ahead of print 2019/01/18; doi 10.1080/20013078.2018.1555419.

52. de Rond L, Coumans FAW, Nieuwland R, van Leeuwen TG, van der Pol E. Deriving extracellular vesicle size from scatter intensities measured by flow cytometry. Curr Protoc Cytom 2018; 86(1): e43; e-pub ahead of print 2018/09/01; doi 10.1002/cpcy.43.

53. Beekman P, Enciso-Martinez A, Rho HS, Pujari SP, Lenferink A, Zuilhof $\mathrm{H}$ et al. Immunocapture of extracellular vesicles for individual multi-modal characterization using AFM, SEM and Raman spectroscopy. Lab Chip 2019; 19(15): 2526-2536; e-pub ahead of print 2019/07/12; doi 10.1039/c9lco0o81j.

54. Enciso-Martinez A, van der Pol E, Lenferink ATM, Terstappen LWMM, Van Leeuwen TG, Otto C. Synchronized Rayleigh and Raman scattering for the characterization of single optically trapped extracellular vesicles. Nanomedicine: Nanotechnology, Biology and Medicine 2019: 102109; doi https://doi.org/10.1016/j.nano.2019.102109.

55. Mathew D, Beekman P, Lemay SG, Zuilhof H, Le Gac S, van der Wiel WG. Electrochemical detection of tumor-derived extracellular vesicles on nano-interdigitated electrodes. Nano Lett 2019; e-pub ahead of print 2019/09/20; doi 10.1021/acs.nanolett.9bo2741.

56. Gool EL, Stojanovic I, Schasfoort RBM, Sturk A, van Leeuwen TG, Nieuwland R et al. Surface Plasmon Resonance is an Analytically Sensitive Method for Antigen Profiling of Extracellular Vesicles. Clin Chem 2017; 63(10): 1633-1641; e-pub ahead of print 2017/08/09; doi 10.1373/ clinchem.2016.271049.

57. de Rond L, van der Pol E, Hau CM, Varga Z, Sturk A, van Leeuwen TG et al. Comparison of Generic Fluorescent Markers for Detection of Extracellular Vesicles by Flow Cytometry. Clin Chem 2018; 64(4): 680-689; e-pub ahead of print 2018/02/18; doi 10.1373/clinchem.2017.278978.

58. de Rond L, Libregts S, Rikkert LG, Hau CM, van der Pol E, Nieuwland Ret al. Refractive index to evaluate staining specificity of extracellular vesicles by flow cytometry. J Extracell Vesicles 2019; 8(1): 1643671; e-pub ahead of print 2019/09/07; doi 10.1080/20013078.2019.1643671. 
59. van der Pol E, de Rond L, Coumans FAW, Gool EL, Boing AN, Sturk A et al. Absolute sizing and label-free identification of extracellular vesicles by flow cytometry. Nanomedicine 2018; 14(3): 801-810; e-pub ahead of print 2018/01/09; doi 10.1016/j.nano.2017.12.012.

60. Nanou A, Miller MC, Zeune LL, de Wit S, Punt CJA, Groen HJM et al. Tumour-derived extracellular vesicles in blood of metastatic cancer patients associate with overall survival. $\mathrm{Br}$ J Cancer 2020; doi 10.1038/s41416-019-0726-9.

61. L G. Rikkert PB, J Caro, F A. Coumans, A Enciso Martinez, G Jenster, S Le Gac, W Lee, Ton G. Van Leeuwen, G B. Loozen, A Nanou, R Nieuwland, H L. Offerhaus, C Otto, D M. Pegtel, M C. Piontek, E Van Der Pol, L De Rond, W H. Roos, R B. Schasfoort, Marca Wauben, H Zuilhof and Leon W. Terstappen. Cancer-ID: towards identification of cancer by tumor-derived extracellular vesicles in blood. Front Oncol 2020; doi 10.3389/fonc.2020.00608.

62. Zeune L. Automated CTC Classification, Enumeration and Pheno Typing: Where Math meets Biology [PhD dissertation]. Enschede: Medical Cell BioPhysics, University of Twente; 2019. 


\title{
Chapter 2
}

\section{Circulating Tumor Cells, tumor-derived Extracellular Vesicles and plasma cytokeratins in castration- resistant prostate cancer patients}

\author{
Afroditi Nanou, Frank A.W. Coumans, Guus van Dalum, Leonie L. Zeune, David \\ Dolling, Wendy Onstenk, Mateus Crespo, Mariane Sousa Fontes, Pasquale Rescigno, \\ Gemma Fowler, Penny Flohr, Christoph Brune, Stefan Sleijfer, Johann S. De Bono and \\ Leon W.M.M. Terstappen
}

Oncotarget $2018,9(27), 19283$

\begin{abstract}
The presence of Circulating Tumor Cells (CTCs) in Castration-Resistant Prostate Cancer (CRPC) patients is associated with poor prognosis. In this study, we evaluated the association of clinical outcome in 129 CRPC patients with CTCs, tumor-derived Extracellular Vesicles (tdEVs) and plasma levels of total (CK18) and caspase-cleaved cytokeratin 18 (ccCK18). CTCs and tdEVs were isolated with the CellSearch system and automatically enumerated. Cut-off values dichotomizing patients into favorable and unfavorable groups were set on a retrospective data set of 84 patients and validated on a prospective data set of 45 patients. Plasma levels of CK18 and ccCK18 were assessed by ELISAs. CTCs, tdEVs and both cytokeratin plasma levels were significantly increased in CRPC patients compared to healthy donors (HDs). All biomarkers except for $\mathrm{CcCK}_{18}$ were prognostic showing a decreased median overall survival for the unfavorable groups of 9.2 vs $21.1,8.1$ vs 23.0 and 10.0 vs 21.5 months respectively. In multivariable Cox regression analysis, tdEVs remained significant. Automated CTC and tdEV enumeration allows fast and reliable scoring eliminating inter- and intraoperator variability. tdEVs provide similar prognostic information to CTC cell counts.
\end{abstract}




\subsection{Introduction}

The presence of Circulating Tumor Cells (CTCs) in Castration-Resistant Prostate Cancer (CRPC) as detected by the CellSearch system is associated with poor outcome compared to patients without detectable CTCs ${ }^{1-6}$. Previously, we showed that the presence of small and large tumor microparticles with or without nucleus, positive for Epithelial Cell Adhesion Molecule (EpCAM) and Cytokeratin (CK) and negative for the leukocyte marker CD45 are also associated with poor outcome in CRPC patients ${ }^{7}$. These tumor microparticles can also be measured using the CellSearch system but do not meet the strigent criteria for CTCs. In the present study, we investigate the clinical relevance of both EpCAM+CK+CD45-tumor microparticles without a nucleus in blood, defined here as tumor-derived Extracellular Vesicles (tdEVs) and soluble cytokeratins in plasma of CRCP patients. Our interest in cytokeratin plasma levels arises from the fact that one of the prerequisites for a cell and an EV isolated by the CellSearch system in blood to be defined as Circulating Tumor Cell (CTC) and tdEV respectively is their cytokeratin expression because of their epithelial origin. Therefore, we additionaly investigated whether soluble cytokeratins present in plasma may be associated with clinical outcome and applied as a surrogate biomarker. Measurements of plasma levels of cytokeratin 18 (CK18) and caspase-cleaved cytokeratin 18 (ccCK18) were performed using M65 and M30 ELISAs respectively ${ }^{8}$. CTCs and tdEVs were imaged with the CellSearch system ${ }^{9}$ and automatically enumerated with ACCEPT software to avoid interoperator variability ${ }^{10}$. Association of CTCs, tdEVs, CK18 and ccCK18 in advanced CRPC patients with clinical outcome was assessed by Kaplan-Meier plots of Overall Survival (OS), uni-, and multi- variable Cox regression analyses. In this study, two data sets were used: a retrospective data set of 84 patients to determine the cut-off values of CTCs and tdEVs for favorable and unfavorable prognosis and a prospective data set of 45 patients to validate the selected cut-off values. The plasma samples of the two data sets were collected in a different way; hence, different cut-off values for CK18 and ccCK18 were used for each one of them.

\subsection{Materials and Methods}

\subsubsection{Patients and healthy donors}

Blood from 16 anonymous HDs was obtained after written informed consent. These samples were used to determine the baseline values of CTCs, tdEVs, CK18 and ccCK18. All patients had histologically confirmed metastatic prostate cancer progressing despite castrate levels of testosterone and had provided written informed consent to trial protocols approved by the institutional review boards at each participating center. Two patient cohorts enrolled in different studies and during different time periods were included in the present study. More specifically, the retrospective data 
set consisted of 84 CRPC patients who were starting a new line of therapy and were enrolled in the IMMC-38 study ${ }^{1}$ out of the 231 evaluable patients of IMMC 38 study, because stored EDTA plasma of only these patients was available for further (cc)CK18 assessment. The digitally stored CellSearch images were also available.

The prospective data set consisted of 45 CRPC patients who were starting a new line of therapy at the Royal Marsden Hospital, and from whom stored plasma samples in CellSave and digitally stored CellSearch images were available.

\subsubsection{Sample collection and preparation}

For the retrospective data set, $7.5 \mathrm{~mL}$ of blood was collected in CellSave blood collection tubes (Menarini, Huntingdon Valley, PA, USA) and 5-10 mL of blood in an EDTA tube. For CTC and tdEV assessment, Cellsave tubes were processed with the CellSearch Autoprep within 96 hours from the time of blood draw. For CK18 and ccCK18 assessment, 5-10 mL EDTA blood was centrifuged for 10 minutes at $1710 \mathrm{~g}$ without brake within 24 hours from the time of blood draw and plasma was collected and stored at $-80^{\circ} \mathrm{C}$ until further use. The plasma samples of the retrospective data set were assessed for $\mathrm{CK} 18$ and $\mathrm{ccCK} 18$ within 5 years from their collection.

For both the prospective data set as well as the healthy donors, $7.5 \mathrm{~mL}$ of blood was collected in CellSave tubes, and was processed within 96 hours after collection. More specifically, the blood samples were centrifuged for 10 minutes at $800 \mathrm{~g}$ without brake and $0.5-2.0 \mathrm{~mL}$ of plasma was collected without disturbing the buffy coat, and stored at $-80^{\circ} \mathrm{C}$ directly after collection until further use. The remaining blood sample was processed with the CellSearch system. The plasma samples of the HDs and the prospective data set were assessed for $\mathrm{CK} 18$ and ccCK18 levels within 1 year from their collection.

\subsubsection{Isolation and detection of CTCs and tdEVs}

The CellSearch system (Menarini, Huntingdon Valley, PA, USA) was used to isolate and detect CTCs and tdEVs. The system consists of the CellTracks Autoprep ${ }^{\circledR}$ and the CellTracks Analyzer $\mathrm{II}^{\circledR}$. The CellTracks Autoprep ${ }^{\circledR}$ immunomagnetically enriches EpCAM+ objects from blood and stains the enriched objects with the nuclear dye DAPI, phycoerythrin conjugated antibodies against cytokeratin 8,18 and 19 (CK-PE) and allophycocyanin conjugated antibody against the leukocyte specific marker CD45 (CD45-APC). The enriched labeled objects are contained in a cartridge, which is placed in a CellTracks Magnest. The CellTracks Analyzer $I I^{\circledR}$ captures digital images with four different fluorescent channels using a 10×/0.45 NA objective and a charge-coupled device camera with $6.7 \times 6.7 \mu \mathrm{m}$ sized pixels. For each cartridge, 144-180 4-layer tiff images of DAPI, FITC, CK-PE, CD45-APC are saved. 


\subsubsection{Enumeration of CTCs and tdEVs}

The CTCs and tdEVs, were enumerated using the open-source ACCEPT software (http://github.com/LeonieZ/ACCEPT) developed in the frames of CTCTrap (www. utwente.nl/en/tnw/ctctrap/) and CANCER-ID EU (www.cancer-id.eu) programs. Briefly, the digitally stored CellSearch fluorescence images are processed by ACCEPT to identify objects using multiscale segmentation ${ }^{10}$. Objects can be categorized using configurable criteria, and objects within a category are shown in a gallery of images as well as in scatter plots using the various parameters measured from the objects. An example of the scatterplots and images of CTCs is shown in Figure 2.1. The scatterplots and images of tdEVs are shown in Supplemental Figure S2.1. Both gates used for CTC and tdEV enumeration are mentioned in the legends of Figure 2.1 and Supplemental Figure S2.1 respectively.

\subsubsection{Measurement of $\mathrm{CK} 18$ and $\mathrm{ccCK} 18$ concentrations}

CK18 present in epithelial cells is cleaved by caspases during apoptosis. Thus, the amount of caspase cleaved CK18 is related to apoptosis, while the total amount of CK18 is related to the sum of CK18 due to apoptosis, necrosis and present in viable cells. Two commercially available ELISA kits, namely M65 and M30-Apoptosense (VLVBio, Nacka, Sweden) were used to measure the levels of soluble ccCK18, and CK18 respectively in plasma samples of healthy donors and CRPC patients. The aforementioned ELISA assays have been already used for clinical assays ${ }^{11-15}$. The M65 assay uses two different monoclonal antibodies to recognize intact and caspase-cleaved $\mathrm{CK} 18$. The $\mathrm{M} 30$ assay uses a monoclonal antibody to recognize the neo-epitope M30, which is exposed after caspase-cleavage of CK18. Both assays have a 96-well plate format and include 7 standards of defined antigen concentrations and both a low and a high concentration quality control (QC). The assays were performed according to manufacturer's instructions. Briefly, technical duplicates of $25 \mu \mathrm{l}$ were added to wells coated with a mouse monoclonal capture antibody. Next, $75 \mu \mathrm{l}$ of Horse Radish Peroxidase HRP-conjugated detection antibody solution was added. After a 2 hour incubation at room temperature (RT) with constant shaking, five sequential washing steps were performed to remove unbound antibody.

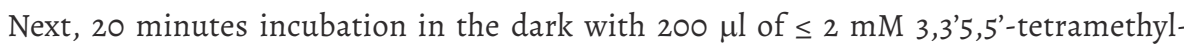
benzidine (TMB) solution resulted in color development proportional to the antigen concentration. The reaction was stopped by the addition of $50 \mu \mathrm{l}$ of $\mathrm{IM}$ sulfuric acid and the $450 \mathrm{~nm}$ absorbance was measured within 5-30 minutes using a microplate reader. All values were corrected for the blank (background) absorbance. After calibration with the standards of defined antigen concentrations, the average of the technical duplicates was converted to concentration in $\mathrm{U} / \mathrm{L}$.

\subsubsection{Statistical Analysis}

Statistical analysis was performed in SPSS 23.0 (SPSS Inc., Chicago, IL, USA) and Stata v15.I (StataCorp, College Station, Texas, USA). To determine whether CTCs, tdEVs, CK18 and ccCK18 can be related to each other through a monotonic function, 
we performed a two-tailed Spearman's Rho test. To assess if there were significant differences between groups (healthy donors vs CRPC patients and retrospective vs prospective data sets) in the examined continuous variables (namely CTCs, tdEVs, CK18 and $\mathrm{ccCK}$ 18), we performed the nonparametric Mann-Whitney U test. To determine the cut-off values to dichotomize the retrospective data set into favorable and unfavorable groups we used Receiver Operating Characteristic (ROC) curves treating survival time as the reference value dichotomized by the median survival time. We set dichotomization cut-off values for CTCs, tdEVs, CK18, and ccCK18 on the value that led to equal sensitivity and specificity (minimum |sensitivity-specificity|). Overall Survival (OS) was defined as the elapsed time in months between blood draw and death. The patients who were lost to follow-up were censored. Median OS was determined by Kaplan-Meier survival curves, and Kaplan-Meier survival curves were compared using the log-rank test. Cox regression models, with each dataset included as a shared frailty parameter, were used to determine univariable and multivariable hazards ratios (HR) for OS with $95 \%$ confidence intervals (CI) for each dichotomized variable.

A final multivariable Cox model with each dataset included as a shared frailty parameter was fit including all characteristics as continuous variables. CK18 and ccCK18 were included with an interaction term for each dataset to account for the different methods in plasma collection. CTC, tdEV, ALP and LDH were log transformed to achieve better model fit. Due to correlation between variables the final model was selected using forward stepwise elimination $\left(\mathrm{p}_{\mathrm{in}}=0.05\right.$ and $\left.\mathrm{p}_{\text {out }}=0.10\right)$. The value of tdEV in the model was assessed by calculating Uno et al's C-Index ${ }^{16}$. Bootstrapping $(\mathrm{n}=1000)$ was used to calculate the $95 \%$ confidence interval and the difference, delta, between c-indeces of each of the models.

\subsection{Results}

\subsubsection{Patient characteristics}

Blood draws of the CRPC patients consisting the retrospective data set (IMMC 38 study) were performed between October 2004 and February 2006. Their average age was 70 years (range $49-87$ years). Blood draws of the CRPC patients consisting the prospective data set were performed between March 2015 and August 2015. Their average age was 68 years (range: 49 - 83 years). The values of serum markers (PSA, $\mathrm{LDH}, \mathrm{ALP}, \mathrm{Alb}, \mathrm{Hb}$ ), age, Gleason score and ECOG performance status for all patients of both data sets are shown in Supplemental Table S2.1. The site of metastases, prior radiation, surgery and treatment of both data sets are summarized in Supplemental Table S2.2. The prospective data set seem to have more advanced disease compared to the retrospective data set, since significantly more patients underwent chemotherapy (91.1\% versus $35.7 \%$ ). Furthermore, $82.2 \%$ and $46.7 \%$ of the patients of the prospective data set were already resistant to abiraterone and enzalutamide respectively, 
indicating more progressed disease compared to the retrospective dataset, where some patients were still undergoing hormone therapy.

\subsubsection{Number of CTCs and tdEVs in $7.5 \mathrm{~mL}$ of blood}

CTCs and tdEVs were enumerated from the stored digital images using ACCEPT software. We compared manually defined CTCs by a human operator using standard Cellsearch criteria for recognition $\left(\mathrm{CK}+, \mathrm{CD}_{45}-\right.$, DAPI,$\left.+>4 \mu \mathrm{m}\right)$ versus the automated CTCs defined by a preconfigured quantitative ACCEPT gate (Figure 2.1) for 129 CRPC patients and 16 healthy controls, see Supplemental Figure S2.2. Linear regression resulted in "automated CTCs" $=0.82$ "manual CTCs" + $0.49\left(\mathrm{R}^{2}=0.882\right)$.
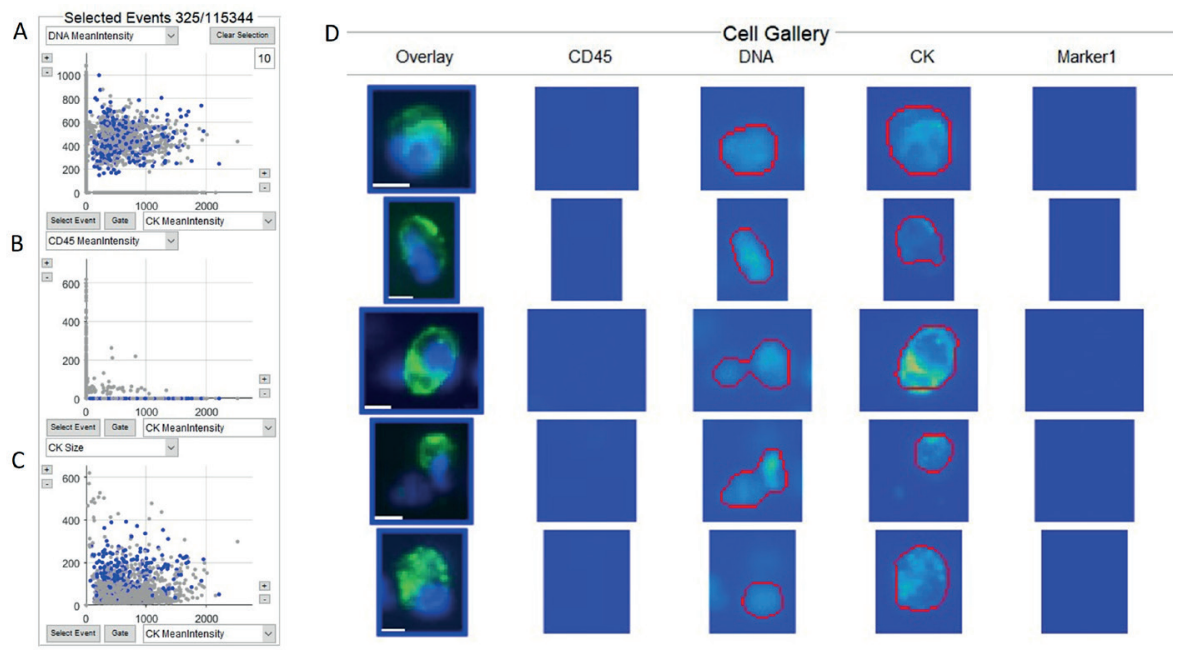

Figure 2.1. ACCEPT display of CTCs identified in a CRPC patient. Three scatterplots (panels A, B, C), where the objects that fall within the definition of the CTC gate (Mean Intensity CD $45 \leq 5$, Mean Intensity DNA $>45$, Mean Intensity $\mathrm{CK}>60$, Mean Intensity Marker $1 \leq 5$, Mean Intensity Marker 2 $\leq 5,16 \leq$ Size $C K \leq 400$, DNA overlay $C K>0.2$ ), are shown as blue dots and those that fall outside of the gate are shown as grey dots. The total number of objects $(115,344)$ identified and the number of objects within the gate (325) are shown on top of Panel A. Panel D shows the thumbnail images of five objects that fall within the gate. The red lines in the thumbnail identifies the area in the image in which ACCEPT identifies contours of the object in each channel. Thumbnails that do not show red contours indicate that no object in this channel could be detected. In the overlay thumbnails, DNA is represented in blue, cytokeratin (CK) in green and CD45 in red. As no CD45 is detected in these images, no red is shown in the overlay. Scale bar indicates $6.4 \mu \mathrm{m}$. 


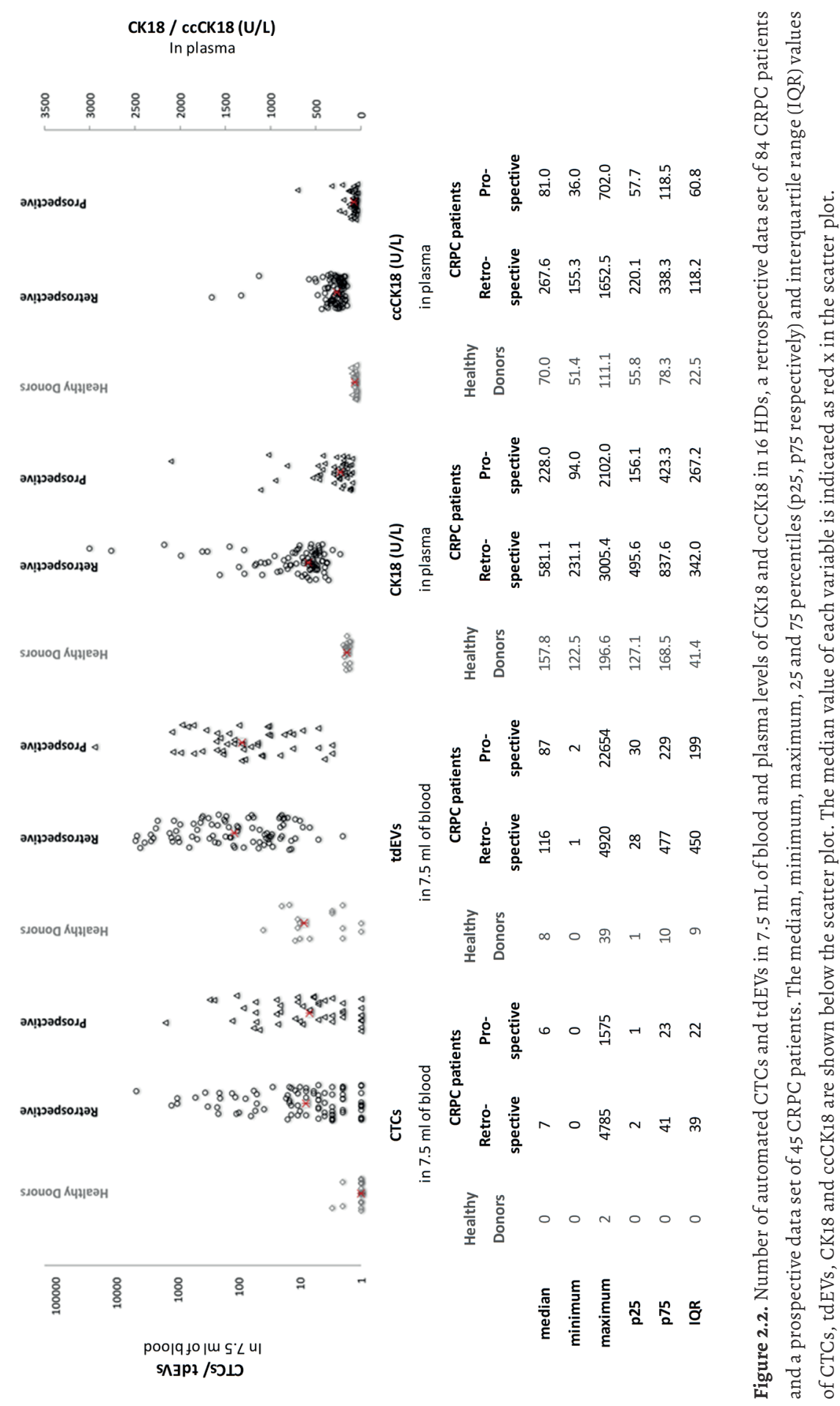


The median, min, max, 25 and 75 percentiles (p25, p75 respectively) and InterQuartile Range (IQR) values of CTCs and tdEVs detected in the 16 healthy controls, the retrospective data set of 84 and the prospective data set of 45 CRPC patients are depicted in Figure 2.2. The number of CTCs and tdEVs were significantly higher in the CRPC patients as compared to HDs (both $\mathrm{p}<0.001$, Mann-Whitney U test). There were no significant differences for CTCs nor for tdEVs between the two data sets $(p=0.81$ for CTCs and $\mathrm{p}=0.32$ for tdEVs, Mann Whitney U test).

Spearman's rho correlation test showed that CTCs and tdEVs of the full data set of 129 CRPC patients were correlated (Spearman's $\mathrm{R}=0.856, \mathrm{p}<0.01$ ). The scatterplot of that data is shown in Supplemental Figure S2.2.

\subsubsection{CK18 and ccCK18 concentrations in plasma samples}

The determined $\mathrm{CK} 18$ and $\mathrm{ccCK} 18$ concentrations of $16 \mathrm{HDs}, 84$ retrospective and 45 prospective CRPC patients are illustrated in Figure 2.2. The concentrations of CK18 and $\mathrm{CCCK}_{18}$ were significantly higher in the CRPC patients compared to the healthy controls ( $\mathrm{p}<0.001$, Mann-Whitney U test). Unexpectedly, the concentrations of CK18 and $\mathrm{ccCK}_{1} 8$ were also significantly higher in retrospective data set as compared to the prospective one ( $\mathrm{p}<0.001$, Mann-Whitney U test). Further investigation (Supplemental Figure S2.4) revealed that the main contributor was the use of EDTA plasma in IMMC38 study versus the CellSave plasma samples in the prospective study.

Given the different values obtained in the two data sets, correlations between CTCs, tdEVs, CK18, ccCK18 and the serum markers of the patients were evaluated within each data set and not in the full data set, Supplemental Table S2.3. Using Spearman's Rho test, CTCs and tdEVs were strongly correlated.

\subsubsection{Determination of cut-off values for CTCs, tdEVs, CK18 and ccCK18}

ROC curves were generated on the retrospective data set to determine the cutoff values for CTCs, tdEVs, CK18, and $\mathrm{ccCK}_{18}$ dichotomization into favorable and unfavorable patient groups for graphical representation. We set dichotomization cutoff values for CTCs, tdEVs, CK18, and $\mathrm{ccCK}_{18}$ on the value that led to equal sensitivity and specificity (value of biomarker for which absolute(sensitivity-specificity) was minimum), Supplemental Figure S2.3. The cut-off values were 5 for CTCs, 105 for tdEVs, $576 \mathrm{U} / \mathrm{L}$ for CK18 and $265 \mathrm{U} / \mathrm{L}$ for ccCK18. tdEVs performed the best in terms of sensitivity and specificity to predict OS of CRPC patients (having the largest AUC and the highest significance), followed by CTCs and CK18.

Because of the large differences of $\mathrm{CK} 18$ and $\mathrm{ccCK} 18$ concentrations between the two data sets, we also determined dichotomization cut-off values on the prospective data set, these were $232 \mathrm{U} / \mathrm{L}$ for CK18 (was $576 \mathrm{U} / \mathrm{L}$ ) and $81 \mathrm{U} / \mathrm{L}$ for CcCK18 (was $265 \mathrm{U} / \mathrm{L}$ ). 

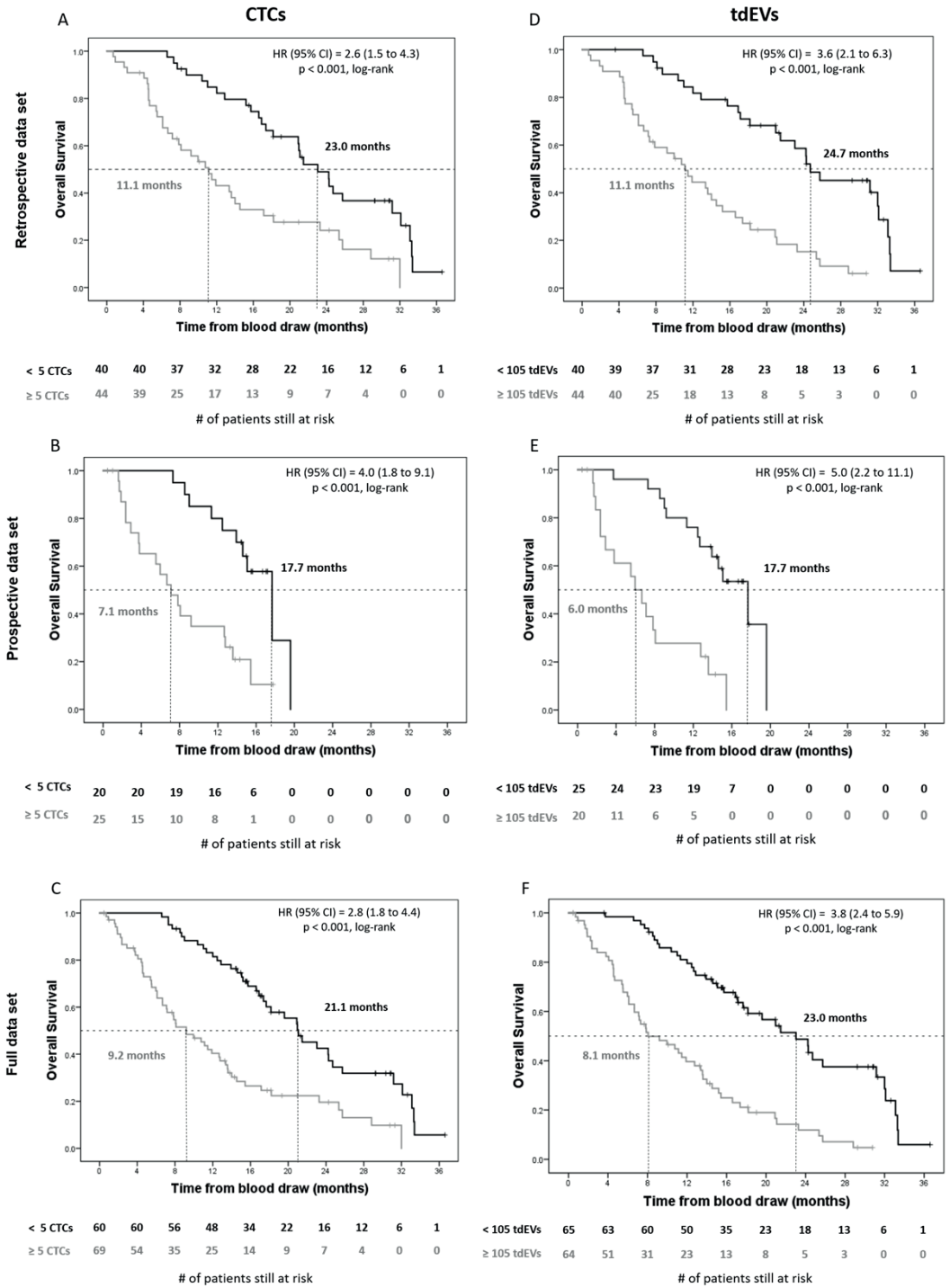

Figure 2.3. Kaplan-Meier plots of overall survival of retrospective, prospective and full data set of CRPC patients for automated CTCs and tdEVs. Kaplan-Meier plots of overall survival of CRPC patients before initiation of therapy for automated CTCs (Panels A-C) and tdEVs (Panels D-F). The retrospective $(n=84)$, prospective $(n=45)$, and full $(n=129)$ data sets are shown in panels $A / D, B / E$, and $\mathrm{C} / \mathrm{F}$ respectively. Patients were dichotomized into unfavorable (grey lines) and favorable groups (black lines) on a cut-off value of 5 for CTCs and 105 for tdEVs in $7.5 \mathrm{~mL}$ of blood. Vertical tick marks indicate censored patients. The number of patients at risk in each group is shown under the horizontal axis. The median overall survival for each group, the Cox hazard ratio (HR), and significance (log-rank p) are indicated in each panel. 

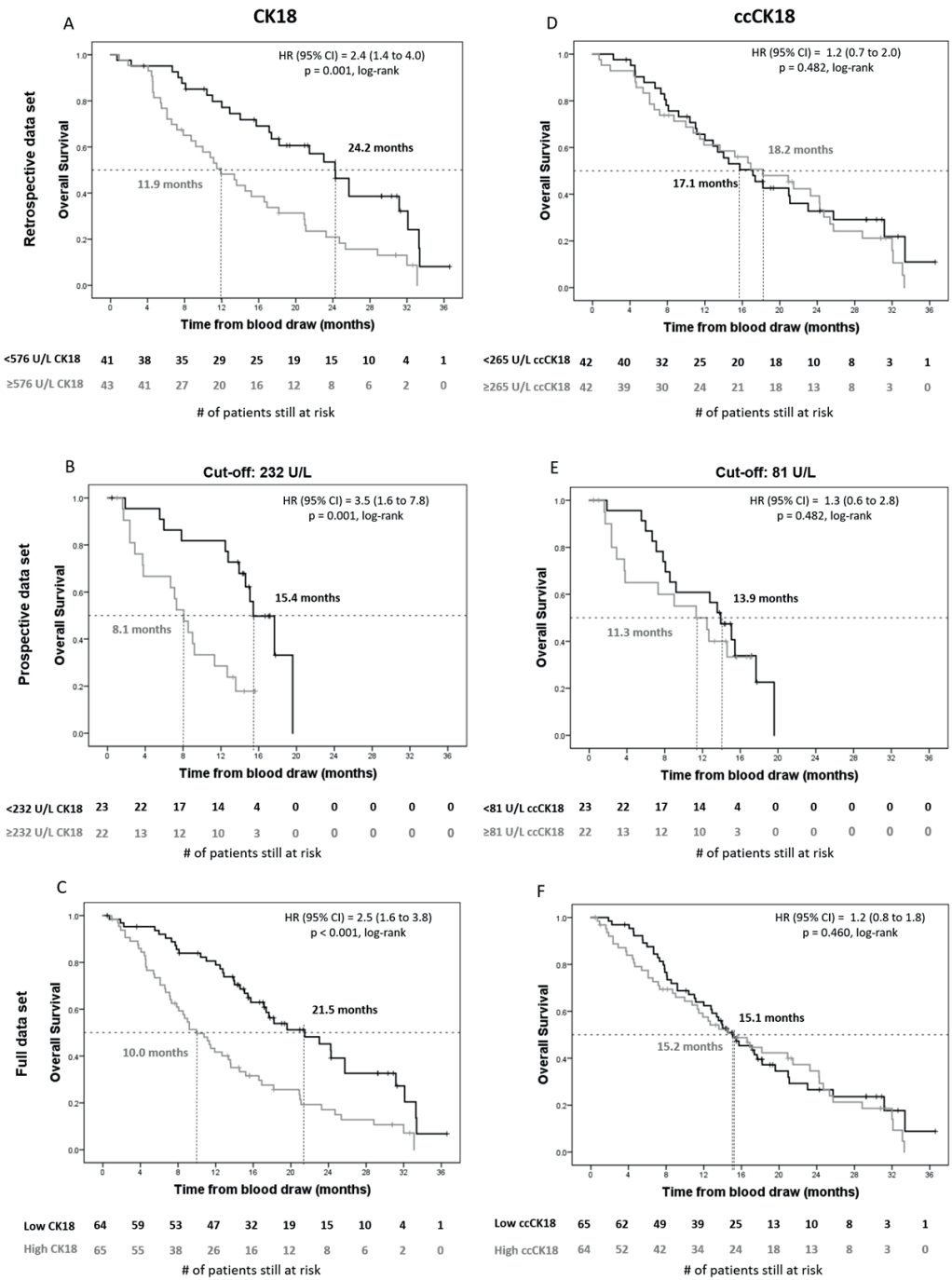

Figure 2.4. Kaplan-Meier plots of overall survival of retrospective, prospective and full data set of CRPC patients for plasma levels of CK18 and cCCK18. Kaplan-Meier plots of overall survival of CRPC patients before initiation of therapy for total plasma cytokeratin 18 (CK18, Panels A-C) and caspasecleaved plasma cytokeratin 18 (ccCK18, Panels D-F) in retrospective ( $\mathrm{n}=84$, Panels A, D), prospective $(n=45$, Panels B, E) and full ( $n=129$, Panels C, F) data sets. Patients were dichotomized into favorable (black line) and unfavorable (grey line) on a cut-off value of $576 \mathrm{U} / \mathrm{L}$ CK18 and $265 \mathrm{U} / \mathrm{L}$ ccCK18 for panels A, D. Patients were dichotomized on a cut-off value of $232 \mathrm{U} / \mathrm{L}$ CK18 and $81 \mathrm{U} / \mathrm{L}$ ccCK18 for panels B,E. Dichotomization on the full data set (Panels C,F) was done using the different cut-off values for each data set. Vertical tick marks indicate censored patients. The number of patients at risk in each group is shown under the horizontal axis. The median overall survival for each group, the Cox hazard ratio (HR), and significance (log-rank p) are indicated in each panel. 


\subsubsection{CTCs, tdEVs, CK18, and ccCK18 versus overall survival of CRPC patients}

Kaplan-Meier plots for patients with favorable and unfavorable CTC and tdEV counts are shown in Figure 2.3. Median OS of patients in the unfavorable CTC group was 9.2 months, versus 21.1 months in the favorable CTC group ( $\mathrm{p}<0.001)$. Similarly, for tdEVs, median OS of patients in the unfavorable tdEV group was 8.1 versus 23.0 months in the favorable tdEV group ( $\mathrm{p}<0.001)$.

Kaplan-Meier plots for $\mathrm{CK} 18$ and ccCK18 are shown in Figure 2.4. The retrospective data set is shown in panels $A / D$. The prospective data set is shown in panels $B / E$, and the full data set is shown in panels $\mathrm{C} / \mathrm{F}$. The two data sets were dichotomized using different cut-off values, because the plasma samples were collected in a different way. However, in both data sets $\mathrm{CK} 18$ was prognostic of OS with the HRs as well as the relative number of patients in favorable and unfavorable groups very similar between the two data sets. For the retrospective data set, the median OS of patients with unfavorable CK18 was 11.9 months, compared to 24.2 months in the favorable group $(p=0.001$ ) with a HR of 2.2. Similarly, for the prospective data set, the patients in the unfavorable CK18 group had significantly lower survival (8.1 versus 15.4 months in the favorable group, $p=0.001$ ) with a HR of 3.5. For ccCK18 no significant difference $(p=0.48)$ in OS could be observed between the two groups regardless the cut-off value used. The summarized HRs of all variables are summarized in Supplemental Table S2.4.

\subsubsection{Multivariable Cox proportional hazards regression analyses}

Table 2.1 shows the final multivariable Cox model selected which included variables CTCs, tdEVs, CK18, prostate specific antigen (PSA), alkaline phosphatase (ALP), lactate dehydrogenase $(\mathrm{LDH})$, albumin $(\mathrm{Alb})$, hemoglobin $(\mathrm{Hb})$ and age. Of the known prognostic variables, $\mathrm{LDH}$, albumin and hemoglobin were all selected in the final model. There was no evidence that either CK18 or ccCK18 were predictive of overall survival after $\mathrm{LDH}$ was included in the multivariable model. CTCs were highly correlated with tdEV (Pearson's rho=0.79 for the transformed variables) and were not included in the final multivariable model after tdEV was the first variable selected. At 24 months, Uno's C-Index was significantly higher in the multivariable model with tdEV (C-Index $=0.77$ ) compared to the multivariable model which included $\mathrm{LDH}$, albumin and hemoglobin (C-Index $=0.73$; Difference $=0.04 ; 95 \% \mathrm{CI}=0.01$ to 0.06 ; $\mathrm{p}=0.006$ ) suggested that $\mathrm{tdEV}$ improved prognostic prediction. There was no evidence of a difference in the prognostic abilities of the model which included CTCs, LDH, albumin and hemoglobin (C-Index=0.77) compared to the model which included tdEVs (Difference=-0.002; 95\% CI: -0.02 to $0.01 ; \mathrm{p}=1.00$ ). 
Table 2.1. Multivariable Cox proportional Hazards Regression analysis for full data set of CRPC patients ( $\mathrm{N}=118$, 11 cases were dropped due to missing values).

\begin{tabular}{lllll}
\hline Variables in equation & HR & $95 \%$ & CI & p-value \\
\hline Albumin $(\mathrm{g} / \mathrm{dl})$ & 0.38 & 0.21 & 0.67 & 0.001 \\
Hemoglobin $(\mathrm{g} / \mathrm{l})$ & 0.81 & 0.69 & 0.94 & 0.006 \\
$\operatorname{tdEV}(\ln (0.0001+\operatorname{count} / 1000))$ & 1.30 & 1.11 & 1.51 & 0.001 \\
$\mathrm{LDH}(\ln (0.001+\mathrm{U} / \mathrm{L} / 100))$ & 1.84 & 1.09 & 3.12 & 0.023 \\
\hline
\end{tabular}

\subsection{Discussion}

Rapid advances in drug development and treatment of cancer patients increase the necessity for new biomarkers to assess their prognosis and response to therapy accurately and in a timely fashion. In the management of CRPC patients, adequate response to therapy is challenging as the traditional Response Evaluation Criteria in Solid Tumors ${ }^{17}$ frequently cannot be applied. The presence or absence of CTCs has emerged as a powerful biomarker to assess prognosis and therapy response ${ }^{1-5}$. Although the CTC numbers measured are extremely low, several ring studies have been conducted and demonstrated the robustness of the test, though a certain level of operator bias cannot be completely eliminated ${ }^{18-21}$. In the original studies conducted with the CellSearch system, patient groups were divided in those with less or more than 5 CTCs per $7.5 \mathrm{~mL}$ of blood and shown to have different clinical outcomes ${ }^{1-5}$. However, recently it was demonstrated that the actual number of CTCs -and not just a count above or below a selected cut-off value- is of importance in order to assess response to therapy ${ }^{4,5,21}$. That fact makes the need for accuracy of the actual CTC count more imperative.

Towards that direction, we previously reported the use of image analysis algorithms to eliminate the operator bias and automatically identify CTCs in the Cellsearch generated images ${ }^{22}$. Efforts to improve these algorithms have continued and have led to the image analysis program ACCEPT. ACCEPT enables a quantitative definition of objects, such as CTCs and tdEVs, derived from the images using specific parameters. In the present study, an excellent correlation $\left(\mathrm{R}^{2}=0.88\right)$ was found (Supplemental Figure S2.2) between the manual (obtained by the operator) and the automated CTC counts (obtained by ACCEPT).

To define the cut-off values dichotomizing patients into groups of higher and lower risk by ACCEPT automated CTC and tdEV counts, a retrospective data set of 84 CRPC patients from the original IMMC38 study was used. ROC analysis in that data set (Supplemental Figure S2.3) showed a cut-off value of 5 CTCs in $7.5 \mathrm{~mL}$ of whole blood that stratified patients into favorable and unfavorable groups (Figure 2.3). The CTC 
cut-off was validated in a prospective data set of 45 newly enrolled CRPC patients. In the full data set, patients of the unfavorable group (with $\geq 5$ CTCs) had significantly shorter median OS compared to patients of the favorable group $(\mathrm{p}<0.001)$ with a HR of 2.8 (95\% CI: 1.8 to 4.4$)$.

In the CellSearch image analysis algorithms, objects expressing both Cytokeratin as well as DAPI are presented to the operator, whereas the majority of tdEVs is missed, as the latter ones do not have DAPI signal. Using the automated ACCEPT identification of tdEVs however, the labor-intensive manual review of the original stack of 144-180 of fluorescence images for each patient sample ${ }^{7}$ can be replaced by a process with perfect repeatability. In our study, there was a strong correlation between automated CTC and tdEV counts (Supplemental Figure S2.2) and their presence in higher amounts was strongly correlated with poor clinical outcome (Figure 2.3).

Since tdEVs were isolated with the CellSearch system, they were enriched from the blood fraction centrifuged at $800 \mathrm{~g}$ based on their EpCAM expression and were detected by the expression of Cytokeratins. However, the tdEV number in the plasma should be much higher because the vast majority of these EVs have a diameter below $4 \mu \mathrm{m}^{23}$. Moreover, the CellSearch Analyzer was not designed for the detection of small particles, and the fraction of tdEVs below the CellSearch detection limit is unknown. Further investigation is recommended for the isolation and detection of tdEVs from plasma of patients and their correlation with clinical outcome.

To assess the clinical relevence of soluble Cytokeratins in plasma, CK18 and ccCK18 were determined by M65 and M30 ELISAs respectively. Several studies have shown the significantly elevated (cc)CK18 levels in serum/plasma of different cancer patients compared to the respective values of healthy donors ${ }^{12,14,24}$. Moreover, CK18 and ccCK18 have been used in several studies to evaluate the induced cell death modes and antitumor activity of different drug treatments $8,11,13,25,26$. Interestingly, in a previous study, both ccCK18 and CK18 levels in plasma were predictive of the clinical outcome of small cell lung cancer patients ${ }^{15}$. In another study on both small and non-small cell lung cancer patients, the serum levels of $\mathrm{ccCK}_{18}$ were prognostic for OS ${ }^{27}$. A third study demonstrated significantly increased levels of $\mathrm{ccCK}_{18} 8$ in the sera of breast cancer patients compared to HDs but without any association of CCCK18 with the OS of these patients ${ }^{24}$. In our study in CRPC patients, we found that both CK18 and ccCK18 are elevated in patients compared to HDs, but only CK18 is prognostic for OS (Figure 2.4). It is worth mentioning that the ELISA assays we applied for CK18 and ccCK18 do not involve a detergent and thus measure only the soluble proteins and do not detect CK18 or ccCK18 inside tdEVs. Adding a detergent to the plasma may solubilize the tdEVs, and potentially result in a prognostic ccCK18 because a portion of cytokeratins in CTCs and tdEVs is caspase-cleaved as shown by M30 expression ${ }^{28}$. 
In summary, our findings suggest that ACCEPT software allows fast enumeration of well-defined objects as CTCs and tdEVs eliminating interoperator bias. The enumeration of tdEVs in CRPC patients can provide prognostic information equivalent to CTCs in CRPC disease. tdEVs were typically detected at a 20-times higher frequencies in $7.5 \mathrm{ml}$ of blood compared to CTCs so tdEVs may offer increased utility. The association between OS and CK18 in CRPC is not as strong as CTCs and tdEVs based on the respective HRs. The multivariable analysis of the full data set of CRPC patients including CTCs, tdEVs, CK18 and CCCK18 and traditional biomarkers such as age, PSA, ALP, LDH, Alb and $\mathrm{Hb}$ resulted in a final model with $\mathrm{LDH}$, albumin, hemoglobin and tdEVs. tdEVs improved significantly the prognostic prediction of the patients. Neither CK18 nor ccCK18 were predictive of OS after LDH inclusion. CTCs were highly correlated with tdEVs so were not present in the final multivariable model but provided equivalent prognostic information. To predict whether a certain treatment will be effective, assessment of the treatment target will need to be assessed. Preferably one would examine tumor cells representing the various metastatic sites, but as that is practically not feasible, CTCs can provide this information provided that one can isolate them in sufficient quantity ${ }^{29,30}$. Due to the fact that most patients have single-digit CTC counts, the higher number of tdEVs could render them to a promising surrogate biomarker for the assessment of changes of tumor load (through their rises and declines) in response to therapy over time and even in personalized therapeutics by proceeding with the downstream analysis of their protein and RNA cargo.

\section{Acknowledgments}

The authors acknowledge the Developmental BioEngineering department of the University of Twente for providing access to their microplate absorbance reader and the Experimental Centre for Technical Medicine (ECTM) of the University of Twente for providing the healthy donor samples. 


\section{References}

1. de Bono JS, Scher HI, Montgomery RB, Parker C, Miller MC, Tissing H et al. Circulating tumor cells predict survival benefit from treatment in metastatic castration-resistant prostate cancer. Clin Cancer Res 2008; 14(19): 6302-6309; doi 10.1158/1078-0432.CCR-08-0872.

2. Scher HI, Heller G, Molina A, Attard G, Danila DC, Jia X et al. Circulating tumor cell biomarker panel as an individual-level surrogate for survival in metastatic castration-resistant prostate cancer. J Clin Oncol 2015; 33(12): 1348-1355; doi 10.1200/JCO.2014.55.3487.

3. Goldkorn A, Ely B, Quinn DI, Tangen CM, Fink LM, Xu T et al. Circulating tumor cell counts are prognostic of overall survival in SWOG SO421: a phase III trial of docetaxel with or without atrasentan for metastatic castration-resistant prostate cancer. J Clin Oncol 2014; 32(11): 11361142; e-pub ahead of print 2014/03/10; doi 10.1200/JCO.2013.51.7417.

4. Lorente D, Olmos D, Mateo J, Bianchini D, Seed G, Fleisher M et al. Decline in Circulating Tumor Cell Count and Treatment Outcome in Advanced Prostate Cancer. Eur Urol 2016; 70(6): 985-992; e-pub ahead of print 2016/06/09; doi 10.1016/j.eururo.2016.05.023.

5. Lorente D, Ravi P, Mehra N, Pezaro C, Omlin A, Gilman A et al. Interrogating Metastatic Prostate Cancer Treatment Switch Decisions: A Multi-institutional Survey. Eur Urol Focus 2016; e-pub ahead of print 2016/10/10; doi 10.1016/j.euf.2016.09.005.

6. Miyamoto DT, Sequist LV, Lee RJ. Circulating tumour cells-monitoring treatment response in prostate cancer. Nat Rev Clin Oncol 2014; 11(7): 401-412; e-pub ahead of print 2014/05/13; doi 10.1038/nrclinonc.2014.82.

7. Coumans FA, Doggen CJ, Attard G, de Bono JS, Terstappen LW. All circulating EpCAM+CK+CD45- objects predict overall survival in castration-resistant prostate cancer. Ann Oncol 2010;21(9): 1851-1857; e-pub ahead of print 2010/02/10; doi 10.1093/annonc/mdq030.

8. Kramer G, Erdal H, Mertens HJ, Nap M, Mauermann J, Steiner G et al. Differentiation between cell death modes using measurements of different soluble forms of extracellular cytokeratin 18. Cancer Res 2004; 64(5): 1751-1756.

9. Allard WJ, Matera J, Miller MC, Repollet M, Connelly MC, Rao C et al. Tumor cells circulate in the peripheral blood of all major carcinomas but not in healthy subjects or patients with nonmalignant diseases. Clin Cancer Res 2004; 10(20): 6897-6904; e-pub ahead of print 2004/10/27; doi 10/20/6897 [pii]10.1158/1078-0432.CCR-04-0378.

10. Zeune L, van Dalum G, Terstappen L, van Gils S, Brune C. Multiscale Segmentation via Bregman Distances and Nonlinear Spectral Analysis. Siam Journal on Imaging Sciences (Article) 2017; 10(1): 111-146; doi 10.1137/16M1074503.

11. de Haas EC, di Pietro A, Simpson KL, Meijer C, Suurmeijer AJ, Lancashire LJ et al. Clinical evaluation of M30 and M65 ELISA cell death assays as circulating biomarkers in a drugsensitive tumor, testicular cancer. Neoplasia 2008; 10(10): 1041-1048.

12. Tas F, Karabulut S, Yildiz I, Duranyildiz D. Clinical significance of serum M30 and M65 levels in patients with breast cancer. Biomed Pharmacother 2014; 68(8): 1135-1140; e-pub ahead of print 2014/10/31; doi 10.1016/j.biopha.2014.10.021. 
13. Cummings J, Hodgkinson C, Odedra R, Sini P, Heaton SP, Mundt KE et al. Preclinical evaluation of M30 and M65 ELISAs as biomarkers of drug induced tumor cell death and antitumor activity. Mol Cancer Ther 2008; 7(3): 455-463; doi 10.1158/1535-7163.MCT-07-2136.

14. Ozturk B, Coskun U, Sancak B, Yaman E, Buyukberber S, Benekli M. Elevated serum levels of M30 and M65 in patients with locally advanced head and neck tumors. Int Immunopharmacol 2009; 9(5): 645-648; e-pub ahead of print 2009/02/26; doi 10.1016/j.intimp.2009.02.004.

15. Hou JM, Greystoke A, Lancashire L, Cummings J, Ward T, Board R et al. Evaluation of circulating tumor cells and serological cell death biomarkers in small cell lung cancer patients undergoing chemotherapy. Am J Pathol 2009; 175(2): 808-816; e-pub ahead of print 2009/07/23; doi 10.2353/ajpath.2009.090078.

16. Uno H, Cai T, Pencina M, D'Agostino R, Wei L. On the C-statistics for evaluating overall adequacy of risk prediction procedures with censored survival data. Statistics in Medicine (Article) 2011; 30(10): 1105-1117; doi 10.1002/sim.4154.

17. Eisenhauer EA, Therasse P, Bogaerts J, Schwartz LH, Sargent D, Ford R et al. New response evaluation criteria in solid tumours: revised RECIST guideline (version 1.1). Eur J Cancer 2009; 45(2): 228-247; doi 10.1016/j.ejca.2008.10.026.

18. Ignatiadis M, Riethdorf S, Bidard FC, Vaucher I, Khazour M, Rothé F etal. International study on inter-reader variability for circulating tumor cells in breast cancer. Breast Cancer Res 2014; 16(2): R43; e-pub ahead of print 2014/04/23; doi 10.1186/bcr3647.

19. Riethdorf S, Fritsche H, Müller V, Rau T, Schindlbeck C, Rack B et al. Detection of circulating tumor cells in peripheral blood of patients with metastatic breast cancer: a validation study of the CellSearch system. Clin Cancer Res 2007; 13(3): 920-928; doi 10.1158/1078-0432.CCR-06-1695.

20. Kraan J, Sleijfer S, Strijbos MH, Ignatiadis M, Peeters D, Pierga JY et al. External quality assurance of circulating tumor cell enumeration using the CellSearch $\left.{ }^{\oplus}\right)$ system: a feasibility study. Cytometry B Clin Cytom 2011; 80(2): 112-118; e-pub ahead of print 2010/11/10; doi 10.1002/ cyto.b.20573.

21. Coumans FA, Ligthart ST, Terstappen LW. Interpretation of changes in circulating tumor cell counts. Transl Oncol 2012; 5(6): 486-491; e-pub ahead of print 2012/12/01.

22. Ligthart ST, Coumans FA, Attard G, Cassidy AM, de Bono JS, Terstappen LW. Unbiased and automated identification of a circulating tumour cell definition that associates with overall survival. PLoS One 2011; 6(11): e27419; e-pub ahead of print 2011/11/07; doi 10.1371/journal. pone.0027419.

23. van der Pol E, Coumans FA, Grootemaat AE, Gardiner C, Sargent IL, Harrison P et al. Particle size distribution of exosomes and microvesicles determined by transmission electron microscopy, flow cytometry, nanoparticle tracking analysis, and resistive pulse sensing. J Thromb Haemost 2014; 12(7): 1182-1192; doi 10.1111/jth.12602.

24. Ueno T, Toi M, Bivén K, Bando H, Ogawa T, Linder S. Measurement of an apoptotic product in the sera of breast cancer patients. Eur J Cancer 2003; 39(6): 769-774.

25. Pichon MF, Labroquère $M$, Rezaï K, Lokiec $F$. Variations of soluble fas and cytokeratin 18-Asp 396 neo-epitope in different cancers during chemotherapy. Anticancer Res 2006; 26(3B): 2387-2392. 
26. Kramer G, Schwarz S, Hagg M, Havelka A, Linder S. Docetaxel induces apoptosis in hormone refractory prostate carcinomas during multiple treatment cycles. British Journal of Cancer (Article) 2006; 94(11): 1592-1598; doi 10.1038/sj.bjc.6603129.

27. Ulukaya E, Yilmaztepe A, Akgoz S, Linder S, Karadag M. The levels of caspase-cleaved cytokeratin 18 are elevated in serum from patients with lung cancer and helpful to predict the survival. Lung Cancer 2007; 56(3): 399-404; e-pub ahead of print 2007/02/20; doi 10.1016/j. lungcan.2007.01.015.

28. Larson CJ, Moreno JG, Pienta KJ, Gross S, Repollet M, O'hara SM et al. Apoptosis of circulating tumor cells in prostate cancer patients. Cytometry A 2004; 62(1): 46-53; doi 10.1002/ cyto.a.20073.

29. Coumans FA, Ligthart ST, Uhr JW, Terstappen LW. Challenges in the enumeration and phenotyping of CTC. Clin Cancer Res 2012; 18(20): 5711-5718; e-pub ahead of print 2012/09/25; doi 10.1158/1078-0432.CCR-12-1585.

30. Stoecklein NH, Fischer JC, Niederacher D, Terstappen LW. Challenges for CTC-based liquid biopsies: low CTC frequency and diagnostic leukapheresis as a potential solution. Expert Rev Mol Diagn 2016; 16(2): 147-164; doi 10.1586/14737159.2016.1123095. 


\section{Supplementary data}

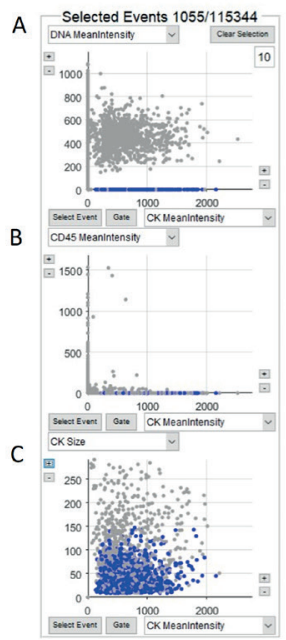

D

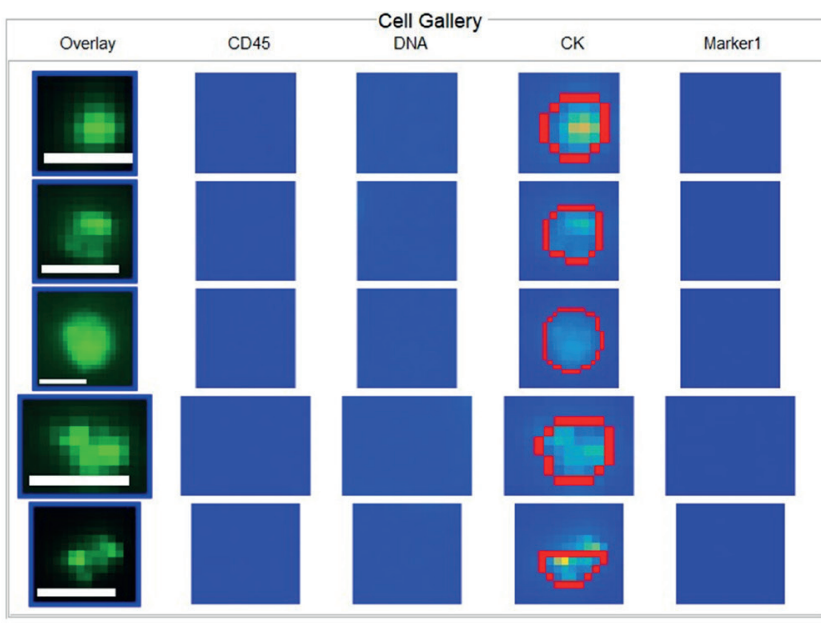

Supplemental Figure S2.1. ACCEPT display of tdEVs identified in a CRPC patient. Same display as used in Figure 2.1 with the difference that the gate for tdEV definition (Mean Intensity $C D 45 \leq 5$, Mean Intensity DNA $\leq 5$, Mean Intensity Marker $1 \leq 5$, Mean Intensity Marker $2 \leq 5$, Mean Intensity CK $>60$, Max Intensity CK > 90, Size CK $\leq 150$, Perimeter CK > , Eccentricity CK $\leq 0.8$, Perimeter to Area $\mathrm{CK} \leq 1$ ) was used on this CRPC patient. The total number of 115,344 objects is the same but the number of objects within the gate is now 1,055 as shown on top of Panel A. Panel D shows the thumbnail images of five objects that fall within the tdEV gate. Scale bar indicates $6.4 \mu \mathrm{m}$. 
A

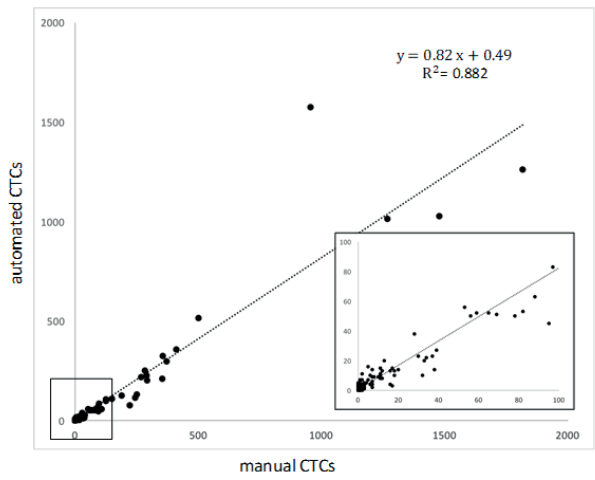

C

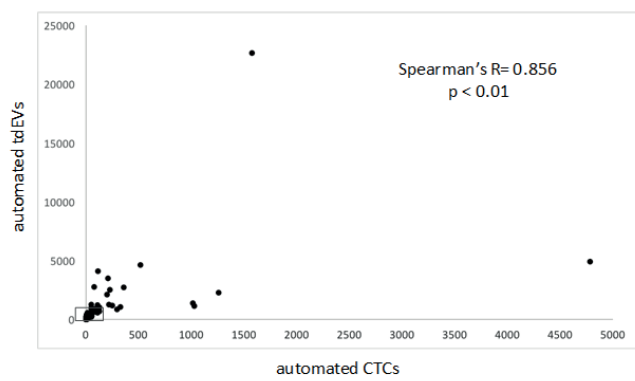

B

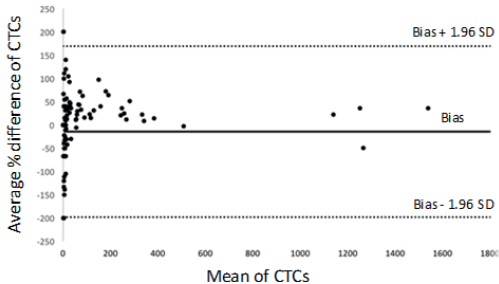

D

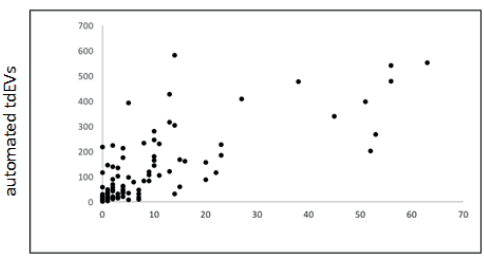

automated CTCs

Supplemental Figure S2.2. Correlation of manual with automated CTCs and automated CTCs with tdEVs using scatterplot and Bland-Altman plot. Scatterplot (panel A) and Bland-Altman Plot (panel B) of manual CTCs (detected by operator definition) and automated CTCs (by ACCEPT enumeration). The scatterplot of the enclosed by square data points of panel $\mathrm{A}$ is shown in higher magnification. Scatterplot (panel C) of automated CTCs and tdEVs of 129 CRPC patients. The amount of CTCs is significantly correlated with the amount of tdEVs (Spearman's rho test $\mathrm{R}=0.856, \mathrm{p}<0.01$ ). The scatterplot of the enclosed by square data points is shown in panel $\mathrm{D}$. 


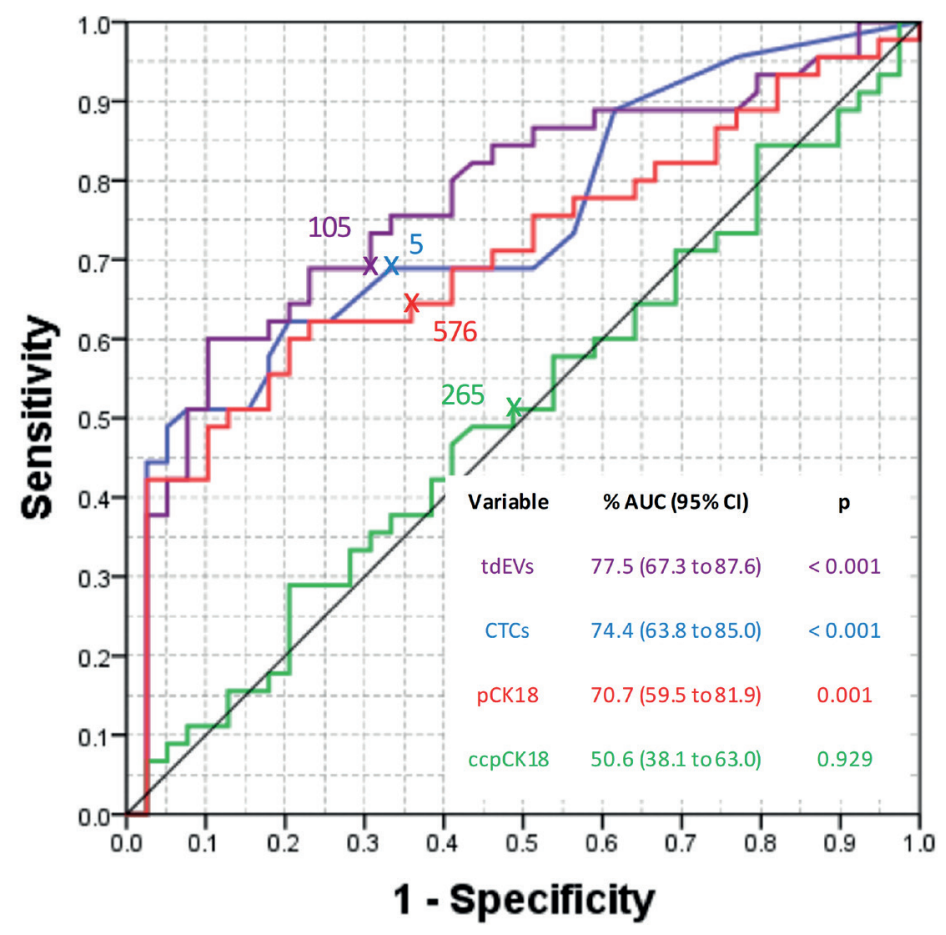

Diagonal segments are produced by ties.

Supplemental Figure S2.3. ROC curves to determine the dichotomization cut-off values for CTCs, tdEVs, CK18 and ccCK18 in the retrospective data set. The $\mathrm{x}$ and the respective number indicates the value where the sensitivity is equal to the specificity for each biomarker. (value of biomarker for which |sensitivity-specificity| is minimum). 

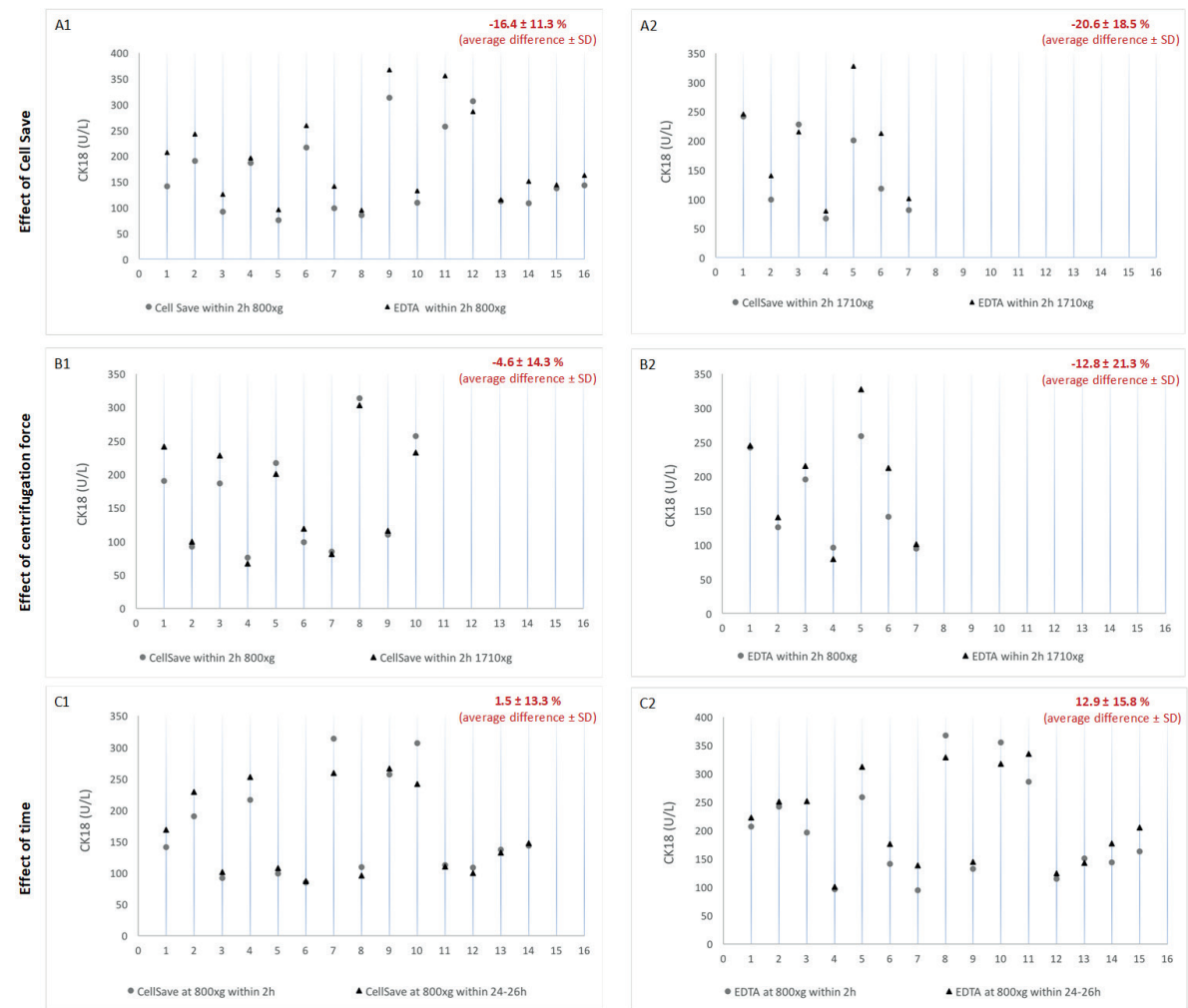

Supplemental Figure S2.4. The concentration of plasma CK18 measured by M65 and ccCK18 measured by $\mathrm{M} 30$ was significantly lower in the prospective data set compared to the retrospective data set. Parameters that could explain this difference were the blood collection tube EDTA versus Cellsave (Panels A1 and A2), the applied centrifugation force of 800xg versus 1710xg (Panels B1 and B2) for the plasma collection and the time between blood draw and plasma collection (Panels $\mathrm{C}_{1}$ and $\mathrm{C} 2$ ). Blood samples of 16 healthy donors were collected in both EDTA and CellSave tubes and the three different parameters were tested on the resulting measured CK18. The largest influence was contributed to the blood collection tube. 
Supplemental Table S2.1. Values of prognostic serum markers (PSA, LDH, ALP, Alb, Hb), age, Gleason score and ECOG performance status of included patients in both retro- and pro-spective data sets. Descriptive statistics (median, mean, SD, min and max) are included for each data set.

Retrospective data set

\begin{tabular}{|c|c|c|c|c|c|c|c|c|}
\hline Patient \# & Age & $\begin{array}{l}\text { Gleason } \\
\text { score }\end{array}$ & ECOG ps & $\begin{array}{l}\text { PSA } \\
\text { (ng/mL) }\end{array}$ & $\begin{array}{l}\text { LDH } \\
(\mathrm{U} / \mathrm{mL})\end{array}$ & $\begin{array}{l}\text { ALP } \\
(\mathrm{U} / \mathrm{mL})\end{array}$ & $\begin{array}{l}\text { Alb } \\
(\mathrm{g} / \mathrm{dL})\end{array}$ & $\begin{array}{l}\mathrm{Hb} \\
(\mathrm{g} / \mathrm{dL})\end{array}$ \\
\hline Ri & 67 & 9 & 0 & 62 & 146 & 228 & & 14.4 \\
\hline R2 & 56 & 7 & 0 & 17.7 & 175 & 90 & & 12.8 \\
\hline R3 & 69 & 6 & 0 & 3796 & 232 & 82 & 4.4 & 10.4 \\
\hline R4 & 62 & 7 & & 62.9 & 467 & 179 & 4 & 10.6 \\
\hline R5 & 60 & 9 & 1 & 29.7 & 215 & 78 & & 12.4 \\
\hline R6 & 58 & 7 & 2 & 1443 & 361 & 409 & 4 & 9.7 \\
\hline R7 & 79 & & 2 & 320 & 939 & 252 & 3.4 & 8.6 \\
\hline R8 & 74 & 9 & 1 & 175 & 384 & 917 & 3.9 & 12.4 \\
\hline R9 & 65 & 8 & 2 & 527 & 324 & 230 & 3.6 & 8.2 \\
\hline Rio & 77 & 8 & 1 & 247 & 243 & 103 & 3.7 & 11.6 \\
\hline R11 & 70 & 8 & 1 & 122 & 285 & 99 & 4.4 & 11.8 \\
\hline R12 & 82 & 5 & 1 & 1634 & 237 & 130 & 3.9 & 9.5 \\
\hline $\mathrm{R} 13$ & 66 & 8 & 0 & 33.3 & 248 & 96 & 4.4 & 13.7 \\
\hline R14 & 81 & 8 & 1 & 129 & 198 & 89 & 4 & 12.7 \\
\hline R15 & 72 & 7 & 2 & 88 & 614 & 83 & 4.4 & 11.9 \\
\hline R16 & 72 & 9 & 1 & 80 & 214 & 129 & 4 & 11.5 \\
\hline R17 & 61 & 8 & 1 & 64 & 225 & 145 & 4.3 & 13.6 \\
\hline R18 & 70 & 7 & 0 & 219 & 411 & 387 & 3.5 & 9.8 \\
\hline R19 & 70 & 7 & 0 & 756 & 224 & 104 & 3.6 & 11.3 \\
\hline R2O & 76 & 7 & 0 & 60 & 220 & 97 & 3.4 & 11.5 \\
\hline R21 & 58 & 7 & 1 & 18.5 & 182 & 126 & 3.8 & 12.3 \\
\hline R22 & 59 & 8 & 0 & 29.8 & 644 & 68 & 3.3 & 11.9 \\
\hline R23 & 58 & 7 & 0 & 76 & 258 & 125 & 3.8 & 13.7 \\
\hline R24 & 78 & 4 & 0 & 268 & 272 & 94 & 3.5 & 15 \\
\hline R25 & 74 & 9 & 1 & 135 & 1117 & 696 & 3.9 & 11.6 \\
\hline R26 & 64 & 9 & 0 & 73 & 193 & 102 & 3.9 & 13.6 \\
\hline R27 & 64 & 7 & 0 & 64 & 298 & 135 & 4.5 & 13.4 \\
\hline R28 & 63 & 9 & 0 & 24.7 & 229 & 63 & 4.1 & 13 \\
\hline R29 & 87 & 7 & 1 & 241 & 305 & 219 & 4.2 & 11.1 \\
\hline R30 & 81 & 8 & 0 & 61 & 188 & 450 & 3.8 & 11.1 \\
\hline R31 & 70 & 7 & 1 & 965 & 659 & 321 & 4 & 9.2 \\
\hline
\end{tabular}




\begin{tabular}{|c|c|c|c|c|c|c|c|c|}
\hline Patient \# & Age & $\begin{array}{l}\text { Gleason } \\
\text { score }\end{array}$ & ECOG ps & $\begin{array}{l}\text { PSA } \\
\text { (ng/mL) }\end{array}$ & $\begin{array}{l}\text { LDH } \\
(\mathrm{U} / \mathrm{mL})\end{array}$ & $\begin{array}{l}\text { ALP } \\
(\mathrm{U} / \mathrm{mL})\end{array}$ & $\begin{array}{l}\text { Alb } \\
(\mathrm{g} / \mathrm{dL})\end{array}$ & $\begin{array}{l}\mathrm{Hb} \\
(\mathrm{g} / \mathrm{dL})\end{array}$ \\
\hline R32 & 76 & 9 & 1 & 47 & 115 & 127 & 4.1 & 13.2 \\
\hline R33 & 72 & 7 & 0 & 481 & 235 & 337 & 3.9 & 14 \\
\hline $\mathrm{R} 34$ & 53 & 6 & 2 & 107 & 348 & 136 & 3.2 & 13.2 \\
\hline R35 & 67 & 7 & 0 & 39.3 & 258 & 223 & 3.4 & 13.4 \\
\hline $\mathrm{R} 36$ & 77 & 9 & 1 & 36 & 157 & 144 & 4 & 13.2 \\
\hline $\mathrm{R} 37$ & 86 & 2 & 1 & 26.5 & 200 & 67 & 3.5 & 12 \\
\hline $\mathrm{R} 38$ & 75 & 7 & 0 & 18.2 & 177 & 92 & 4.2 & 13.2 \\
\hline R39 & 82 & 6 & 1 & 49 & 264 & 170 & 3.5 & 12 \\
\hline $\mathrm{R} 40$ & 85 & 8 & 2 & 127 & 660 & 180 & 3.5 & 10.3 \\
\hline $\mathrm{R} 41$ & 65 & 10 & 0 & 580 & 533 & 51 & 3.9 & 11.5 \\
\hline $\mathrm{R} 42$ & 79 & 8 & 1 & 82 & 270 & 169 & 3.8 & 13.7 \\
\hline $\mathrm{R} 43$ & 81 & 6 & 0 & 90 & & & 3.5 & 11.1 \\
\hline $\mathrm{R} 44$ & 63 & 7 & 0 & 28.7 & 159 & 47 & 3.8 & 13 \\
\hline R45 & 81 & 5 & 1 & 285 & 114 & 205 & 4.1 & 12.1 \\
\hline $\mathrm{R} 46$ & 72 & 2 & 0 & 107 & 158 & 65 & 4.1 & 12.7 \\
\hline $\mathrm{R} 47$ & 75 & 9 & 0 & 786 & 188 & 86 & 3.5 & 11.7 \\
\hline $\mathrm{R} 48$ & 56 & 9 & 0 & 36.1 & 169 & 81 & 3.6 & 11 \\
\hline R49 & 68 & 9 & 1 & 114 & 206 & 186 & 3.7 & 12 \\
\hline R50 & 74 & 8 & 1 & 178 & 222 & 99 & 4.3 & 14.3 \\
\hline $\mathrm{R}_{51}$ & 79 & 7 & 1 & 1118 & 272 & 165 & & 12.3 \\
\hline $\mathrm{R}_{52}$ & 87 & 7 & 1 & 3630 & 311 & 227 & 3.6 & 13.6 \\
\hline R53 & 63 & 9 & 0 & 37.8 & 247 & 385 & 3.6 & 12.2 \\
\hline R54 & 68 & 8 & 1 & 227 & 182 & 585 & & 9.5 \\
\hline R55 & 85 & 7 & 1 & 604 & 1040 & 352 & 2.8 & 8.7 \\
\hline R56 & 80 & 7 & 0 & 17.4 & 208 & 79 & 3.8 & 11.3 \\
\hline R57 & 76 & 9 & 1 & 69 & 146 & 87 & 3.3 & 13.5 \\
\hline R58 & 70 & & 0 & 58 & 181 & 170 & 4 & 11 \\
\hline R59 & 71 & 7 & 0 & 23.6 & 237 & 124 & 4.4 & \\
\hline R6o & 63 & 8 & 0 & 334 & 206 & 67 & 4.5 & 12.7 \\
\hline R61 & 81 & 8 & 0 & 11.3 & 234 & 85 & 4.7 & 15.6 \\
\hline R62 & 80 & 8 & 0 & 491 & 204 & 63 & 4.5 & 14.8 \\
\hline R63 & 77 & 6 & 1 & 86 & 205 & 85 & 4.3 & 13.1 \\
\hline R64 & 68 & 6 & 1 & 49 & 255 & 120 & 3.9 & 13.1 \\
\hline R65 & 60 & 7 & 0 & 210 & 168 & 182 & 3.7 & 13.6 \\
\hline R66 & 86 & & 0 & 10.5 & 260 & 118 & 4.4 & 14.7 \\
\hline R67 & 75 & & 0 & 186 & 138 & 250 & 3.9 & 14 \\
\hline
\end{tabular}




\begin{tabular}{|c|c|c|c|c|c|c|c|c|}
\hline Patient \# & Age & $\begin{array}{l}\text { Gleason } \\
\text { score }\end{array}$ & ECOG ps & $\begin{array}{l}\text { PSA } \\
\text { (ng/mL) }\end{array}$ & $\begin{array}{l}\text { LDH } \\
(\mathrm{U} / \mathrm{mL})\end{array}$ & $\begin{array}{l}\text { ALP } \\
(\mathrm{U} / \mathrm{mL})\end{array}$ & $\begin{array}{l}\text { Alb } \\
(\mathrm{g} / \mathrm{dL})\end{array}$ & $\begin{array}{l}\mathrm{Hb} \\
(\mathrm{g} / \mathrm{dL})\end{array}$ \\
\hline R68 & 75 & 8 & 0 & 19.2 & 284 & 164 & 4.2 & 9.4 \\
\hline R69 & 69 & & 0 & 435 & 263 & 103 & 3.8 & 13 \\
\hline R70 & 69 & 6 & 0 & 90 & 219 & 46 & 4.2 & 13.4 \\
\hline R71 & 60 & 9 & 1 & 29.2 & 347 & 45 & 4.5 & 11.3 \\
\hline R72 & 58 & 8 & 1 & 5842 & 436 & 495 & 3.5 & 12.1 \\
\hline R73 & 61 & 6 & 0 & 107 & 212 & 106 & 4.4 & 13.4 \\
\hline R74 & 60 & 9 & 0 & 10.1 & 203 & 103 & 4.4 & 13.1 \\
\hline R75 & 65 & 8 & 1 & 551 & 222 & 287 & 3.7 & 9.5 \\
\hline R76 & 49 & 8 & 1 & 27.3 & 210 & 118 & 3.7 & 11.9 \\
\hline R77 & 65 & 7 & 1 & 23.9 & 185 & 82 & 39 & 13.5 \\
\hline R78 & 58 & 7 & 0 & 73 & 167 & 144 & 4.2 & 14.2 \\
\hline R79 & 57 & 6 & 1 & 512 & 173 & 310 & 4 & 13.54 \\
\hline $\mathrm{R} 80$ & 75 & & & 475 & 244 & 240 & 3.2 & 11.5 \\
\hline R81 & 62 & 8 & 0 & 29.8 & 157 & 78 & 4.8 & 14.8 \\
\hline R82 & 56 & 7 & 1 & 683 & 2092 & 1801 & 3.1 & 10.7 \\
\hline R83 & 61 & 9 & 2 & 1162 & 234 & 181 & 4.3 & 11.4 \\
\hline R84 & 64 & 9 & 1 & 20.9 & 310 & 223 & 3.5 & 13.6 \\
\hline Mean & 69.8 & 7.4 & 0.6 & 384.4 & 308.3 & 198.3 & 4.3 & 12.2 \\
\hline SD & 9.1 & 1.5 & 0.6 & 868.7 & 271.5 & 231.5 & 4 & 1.6 \\
\hline Median & 70 & 7.5 & 1 & 89 & 232 & 127 & 3.9 & 12.3 \\
\hline Min & 49 & 2 & 0 & 10.1 & 114 & 45 & 2.8 & 8.2 \\
\hline Max & 87 & 10 & 2 & 5842 & 2092 & 1801 & 39 & 15.6 \\
\hline
\end{tabular}

Prospective data set

\begin{tabular}{lllllllll}
\hline Patient \# & Age & $\begin{array}{l}\text { Gleason } \\
\text { score }\end{array}$ & ECOG ps & $\begin{array}{l}\text { PSA (ng/ } \\
\mathrm{mL})\end{array}$ & $\begin{array}{l}\text { LDH }(\mathrm{U} / \\
\mathrm{mL})\end{array}$ & $\begin{array}{l}\text { ALP } \\
(\mathrm{U} / \mathrm{mL})\end{array}$ & $\begin{array}{l}\text { Alb } \\
(\mathrm{g} / \mathrm{dL})\end{array}$ & $\begin{array}{l}\text { Hb } \\
(\mathrm{g} / \mathrm{dL})\end{array}$ \\
\hline P1 & 75 & 6 & 1 & 16 & 256 & 82 & 3.3 & 13 \\
P2 & 68 & 7 & 1 & 220 & 356 & 93 & 3.9 & 10.9 \\
P3 & 75 & 6 & 1 & 28 & 140 & 56 & 3.4 & 12.5 \\
P4 & 83 & 8 & 1 & 280 & 146 & 75 & 3.1 & 11.1 \\
P5 & 60 & 9 & 2 & 56 & 199 & 243 & 3.3 & 10.2 \\
P6 & 69 & 8 & 1 & 623 & 160 & 93 & 3.5 & 10.4 \\
P7 & 62 & 7 & 0 & 111 & 202 & 72 & 3.8 & 12.1 \\
P8 & 71 & 9 & 1 & 23.4 & 207 & 93 & 3.9 & 12.4 \\
P9 & 60 & 7 & 1 & 204 & 553 & 213 & 3.3 & 10.2 \\
P10 & 70 & 7 & 1 & 11 & 156 & 64 & 3.3 & 11 \\
\hline
\end{tabular}




\begin{tabular}{|c|c|c|c|c|c|c|c|c|}
\hline Patient \# & Age & $\begin{array}{l}\text { Gleason } \\
\text { score }\end{array}$ & ECOG ps & $\begin{array}{l}\text { PSA (ng/ } \\
\mathrm{mL})\end{array}$ & $\begin{array}{l}\mathrm{LDH}(\mathrm{U} / \\
\mathrm{mL})\end{array}$ & $\begin{array}{l}\text { ALP } \\
(\mathrm{U} / \mathrm{mL})\end{array}$ & $\begin{array}{l}\text { Alb } \\
\text { (g/dL) }\end{array}$ & $\begin{array}{l}\mathrm{Hb} \\
(\mathrm{g} / \mathrm{dL})\end{array}$ \\
\hline$P_{11}$ & 61 & 7 & 2 & 154 & 314 & 2130 & 3.3 & 10.7 \\
\hline $\mathrm{P}_{12}$ & 55 & 8 & 1 & 26 & 322 & 787 & 2.9 & 10.9 \\
\hline $\mathrm{P}_{13}$ & 71 & 9 & 2 & 152 & 209 & 115 & 3.1 & 10 \\
\hline $\mathrm{P}_{14}$ & 75 & 7 & 1 & 26 & 150 & 77 & 3.7 & 11.5 \\
\hline $\mathrm{P}_{15}$ & 76 & 6 & 1 & 5.9 & 514 & 103 & 3.3 & 11 \\
\hline P16 & 74 & 7 & 0 & 100 & 237 & 46 & 4.1 & 13.2 \\
\hline $\mathrm{P}_{17}$ & 68 & 9 & 1 & 6 & 155 & 64 & 3.6 & 9.5 \\
\hline $\mathrm{P}_{18}$ & 71 & 10 & 1 & 101 & 343 & 99 & 3.5 & 12.1 \\
\hline$P_{19}$ & 72 & 9 & 1 & 15.4 & 215 & 162 & 2.9 & 10.1 \\
\hline P2O & 65 & 9 & 0 & 120 & 185 & 162 & 4.1 & 13.6 \\
\hline $\mathrm{P}_{21}$ & 75 & 7 & 0 & 17 & 219 & 75 & 4.1 & 15.8 \\
\hline P22 & 62 & 9 & 0 & 129 & 174 & 47 & 4.4 & 13.9 \\
\hline $\mathrm{P}_{23}$ & 73 & 10 & 1 & 70 & 176 & 123 & 3.2 & 9.6 \\
\hline P24 & 77 & 7 & 1 & 34 & 203 & 186 & 3.8 & 12.6 \\
\hline P25 & 63 & 6 & 1 & 73 & 148 & 52 & 3.7 & 8.6 \\
\hline P26 & 79 & & 2 & 180 & 1786 & 1282 & 2.3 & 9 \\
\hline P27 & 67 & 9 & 1 & 70 & 391 & 146 & 3.7 & 11.1 \\
\hline P28 & 68 & & 1 & 2510 & 140 & 166 & 3.3 & 12.7 \\
\hline P29 & 76 & 9 & 1 & & 429 & 717 & 3.2 & 10.4 \\
\hline $\mathrm{P}_{30}$ & 53 & & 1 & 8.8 & 201 & 73 & 3.6 & 9.9 \\
\hline$P_{31}$ & 71 & 9 & 1 & 95 & 371 & 67 & 3.2 & 12.1 \\
\hline$P_{32}$ & 75 & & 1 & & 234 & 236 & 3.6 & 11.5 \\
\hline$P_{33}$ & 56 & 9 & 2 & 161 & 313 & 61 & 3.2 & 11.3 \\
\hline P34 & 69 & 9 & 2 & 130 & 508 & 1425 & 4 & 9.4 \\
\hline$P_{35}$ & 62 & & 1 & 32 & 257 & 305 & 4.3 & 12.3 \\
\hline$P_{36}$ & 71 & 7 & 1 & 162 & 520 & 88 & 3.2 & 11.2 \\
\hline$P_{37}$ & 56 & 7 & 0 & 86 & 173 & 59 & 3.3 & 11.4 \\
\hline$P_{38}$ & 68 & 9 & 1 & 115 & 197 & 104 & 3.4 & 12.5 \\
\hline P39 & 71 & 10 & 1 & 5 & 188 & 79 & 3.3 & 8.6 \\
\hline $\mathrm{P}_{40}$ & 65 & & 1 & & 568 & 4027 & 3.7 & 11.5 \\
\hline $\mathrm{P}_{41}$ & 64 & 7 & 1 & 22 & 268 & 82 & 3.7 & 12.3 \\
\hline $\mathrm{P}_{42}$ & 61 & 9 & & 750 & 170 & 54 & 3.9 & 12.5 \\
\hline $\mathrm{P}_{43}$ & 67 & 9 & 1 & 338 & 395 & 81 & 3.5 & 11.5 \\
\hline $\mathrm{P}_{44}$ & 54 & 8 & 0 & 214 & 169 & 272 & 3.8 & 13.7 \\
\hline $\mathrm{P}_{45}$ & 49 & & 1 & 133 & 238 & 86 & 4 & 8.1 \\
\hline Mean & 67.4 & 8 & 1 & 181.3 & 299 & 327.2 & 3.5 & 11.3 \\
\hline
\end{tabular}




\begin{tabular}{lllllllll}
\hline Patient \# & Age & $\begin{array}{l}\text { Gleason } \\
\text { score }\end{array}$ & ECOG ps & $\begin{array}{l}\text { PSA (ng/ } \\
\mathbf{m L})\end{array}$ & $\begin{array}{l}\text { LDH }(\mathrm{U} / \\
\mathbf{m L})\end{array}$ & $\begin{array}{l}\text { ALP } \\
(\mathbf{U} / \mathrm{mL})\end{array}$ & $\begin{array}{l}\text { Alb } \\
(\mathrm{g} / \mathrm{dL})\end{array}$ & $\begin{array}{l}\text { Hb } \\
(\mathrm{g} / \mathrm{dL})\end{array}$ \\
\hline SD & 7.6 & 1.2 & 0.5 & 397.6 & 257.3 & 697.3 & 0.4 & 1.6 \\
Median & 68 & 8 & 1 & 97.5 & 215 & 93 & 3.5 & 11.3 \\
Min & 49 & 6 & 0 & 5 & 140 & 46 & 2.3 & 8.1 \\
Max & 83 & 10 & 2 & 2510 & 1786 & 4027 & 4.4 & 15.8 \\
\hline
\end{tabular}

SD: Standard Deviation, min: minimum, max: maximum, PSA: Prostate Specific Antigen, LDH: Lactate DeHydrogenase, ALP: ALkaline Phosphatase, Hb: Hemoglobin, Alb: Albumin, ECOG ps: Eastern Cooperative Oncology Group performance status

Supplemental Table S2.2. Site of metastases and prior treatments of patients of each data set. Percentages of patients with bone, visceral metastases and prior radiation, surgery and chemotherapy are included at the end of each data set

Retrospective data set

\begin{tabular}{|c|c|c|c|c|c|c|}
\hline Patient \# & $\begin{array}{l}\text { Site of } \\
\text { metastases }\end{array}$ & $\begin{array}{l}\text { Prior } \\
\text { radiation }\end{array}$ & $\begin{array}{l}\text { Prior } \\
\text { surgery }\end{array}$ & $\begin{array}{l}\text { Prior } \\
\text { chemotherapy }\end{array}$ & Abiraterone & Enzalutamide \\
\hline $\mathrm{R} 1$ & bone & no & yes & no & no & no \\
\hline $\mathrm{R} 2$ & bone and visceral & yes & yes & no & no & no \\
\hline R3 & bone and visceral & yes & no & yes & no & no \\
\hline $\mathrm{R} 4$ & bone and visceral & yes & yes & no & no & no \\
\hline R5 & visceral & yes & yes & no & no & no \\
\hline R6 & bone and visceral & yes & yes & no & no & no \\
\hline R7 & bone and visceral & yes & no & yes & no & no \\
\hline $\mathrm{R} 8$ & bone & yes & yes & yes & no & no \\
\hline R9 & bone and visceral & yes & yes & yes & no & no \\
\hline Rio & bone & no & yes & yes & no & no \\
\hline R11 & visceral & yes & yes & no & no & no \\
\hline $\mathrm{R} 12$ & bone & yes & yes & no & no & no \\
\hline $\mathrm{R} 13$ & bone & yes & yes & no & no & no \\
\hline R14 & bone and visceral & yes & no & no & no & no \\
\hline $\mathrm{R} 15$ & bone and visceral & yes & yes & no & no & no \\
\hline R16 & bone & no & no & yes & no & no \\
\hline $\mathrm{R} 17$ & bone & yes & yes & yes & no & no \\
\hline R18 & bone and visceral & yes & yes & yes & no & no \\
\hline R19 & bone & no & no & yes & no & no \\
\hline $\mathrm{R} 2 \mathrm{O}$ & bone & yes & yes & yes & no & no \\
\hline $\mathrm{R} 21$ & bone and visceral & yes & yes & no & no & no \\
\hline
\end{tabular}




\begin{tabular}{|c|c|c|c|c|c|c|}
\hline Patient \# & $\begin{array}{l}\text { Site of } \\
\text { metastases }\end{array}$ & $\begin{array}{l}\text { Prior } \\
\text { radiation }\end{array}$ & $\begin{array}{l}\text { Prior } \\
\text { surgery }\end{array}$ & $\begin{array}{l}\text { Prior } \\
\text { chemotherapy }\end{array}$ & Abiraterone & Enzalutamide \\
\hline $\mathrm{R} 22$ & bone and visceral & yes & yes & yes & no & no \\
\hline R23 & bone & yes & yes & yes & no & no \\
\hline $\mathrm{R} 24$ & bone and visceral & yes & yes & yes & no & no \\
\hline $\mathrm{R} 25$ & bone & yes & yes & yes & no & no \\
\hline $\mathrm{R} 26$ & bone and visceral & no & yes & yes & no & no \\
\hline $\mathrm{R} 27$ & bone & no & yes & no & no & no \\
\hline $\mathrm{R} 28$ & no & no & no & no & no & no \\
\hline R29 & bone & no & yes & no & no & no \\
\hline R30 & bone & no & no & no & no & no \\
\hline R31 & bone & no & no & no & no & no \\
\hline R32 & bone & yes & yes & no & no & no \\
\hline R33 & bone & no & yes & no & no & no \\
\hline R34 & bone & yes & yes & yes & no & no \\
\hline R35 & bone and visceral & yes & no & yes & no & no \\
\hline R36 & bone & no & no & no & no & no \\
\hline R37 & no & yes & yes & no & no & no \\
\hline R38 & bone & no & yes & no & no & no \\
\hline R39 & bone & yes & yes & yes & no & no \\
\hline $\mathrm{R} 40$ & bone and visceral & yes & yes & yes & no & no \\
\hline R41 & bone & no & no & yes & no & no \\
\hline $\mathrm{R} 42$ & $? /$ visceral & yes & no & no & no & no \\
\hline $\mathrm{R} 43$ & bone & no & yes & no & no & no \\
\hline R44 & bone & no & yes & yes & no & no \\
\hline $\mathrm{R} 45$ & bone & yes & yes & no & no & no \\
\hline $\mathrm{R} 46$ & bone & yes & yes & yes & no & no \\
\hline R47 & bone and visceral & yes & no & no & no & no \\
\hline $\mathrm{R} 48$ & visceral & yes & yes & no & no & no \\
\hline R49 & bone & no & no & no & no & no \\
\hline R50 & bone & yes & no & yes & no & no \\
\hline $\mathrm{R}_{51}$ & bone & no & yes & no & no & no \\
\hline R52 & bone & no & yes & no & no & no \\
\hline R53 & bone & yes & yes & no & no & no \\
\hline R54 & bone & yes & yes & no & no & no \\
\hline R55 & bone & yes & no & no & no & no \\
\hline R56 & bone & no & yes & no & no & no \\
\hline R57 & bone and visceral & no & yes & yes & no & no \\
\hline
\end{tabular}




\begin{tabular}{|c|c|c|c|c|c|c|}
\hline Patient \# & $\begin{array}{l}\text { Site of } \\
\text { metastases }\end{array}$ & $\begin{array}{l}\text { Prior } \\
\text { radiation }\end{array}$ & $\begin{array}{l}\text { Prior } \\
\text { surgery }\end{array}$ & $\begin{array}{l}\text { Prior } \\
\text { chemotherapy }\end{array}$ & Abiraterone & Enzalutamide \\
\hline $\mathrm{R} 58$ & bone & no & yes & yes & no & no \\
\hline $\mathrm{R}_{59}$ & bone & yes & no & no & no & no \\
\hline $\mathrm{R} 60$ & bone & yes & yes & yes & no & no \\
\hline R61 & no & no & no & no & no & no \\
\hline R62 & bone & yes & yes & no & no & no \\
\hline R63 & bone & yes & yes & no & no & no \\
\hline R64 & bone & yes & no & no & no & no \\
\hline R65 & bone and visceral & no & no & no & no & no \\
\hline R66 & bone & no & yes & no & no & no \\
\hline R67 & bone & no & yes & no & no & no \\
\hline R68 & bone & yes & yes & no & no & no \\
\hline R69 & bone and visceral & no & yes & no & no & no \\
\hline R70 & bone & yes & yes & no & no & no \\
\hline R71 & visceral & yes & yes & yes & no & no \\
\hline $\mathrm{R} 72$ & bone and visceral & no & no & no & no & no \\
\hline R73 & bone & no & yes & no & no & no \\
\hline R74 & bone and visceral & no & no & yes & no & no \\
\hline R75 & bone and visceral & yes & no & no & no & no \\
\hline R76 & bone and visceral & yes & no & yes & no & no \\
\hline R77 & ?/no visceral & yes & yes & no & no & no \\
\hline R78 & bone & yes & yes & no & no & no \\
\hline R79 & bone & no & yes & no & no & no \\
\hline R80 & no & yes & yes & no & no & no \\
\hline $\mathrm{R} 81$ & bone and visceral & no & yes & no & no & no \\
\hline $\mathrm{R} 82$ & bone & no & yes & no & no & no \\
\hline $\mathrm{R} 83$ & bone & yes & no & yes & no & no \\
\hline $\mathrm{R} 84$ & bone & yes & yes & no & no & no \\
\hline \multirow[t]{4}{*}{ Summary } & $88.1 \%$ bone & $60.7 \%$ yes & $70.2 \%$ yes & $35.70 \%$ & ०\% yes & ०\% yes \\
\hline & metastases & & & & & \\
\hline & $34.5 \%$ visceral & & & & & \\
\hline & metastases & & & & & \\
\hline
\end{tabular}




\begin{tabular}{|c|c|c|c|c|c|c|}
\hline \multicolumn{7}{|c|}{ Prospective data set } \\
\hline Patient \# & Site of metastases & $\begin{array}{l}\text { Prior } \\
\text { radiation }\end{array}$ & $\begin{array}{l}\text { Prior } \\
\text { surgery }\end{array}$ & $\begin{array}{l}\text { Prior } \\
\text { chemotherapy }\end{array}$ & Abiraterone & Enzalutamide \\
\hline P1 & bone & yes & no & yes & yes & yes \\
\hline P2 & bone & no & no & yes & yes & yes \\
\hline $\mathrm{P}_{3}$ & bone and visceral & yes & no & yes & yes & yes \\
\hline $\mathrm{P}_{4}$ & bone & no & no & yes & yes & no \\
\hline $\mathrm{P}_{5}$ & bone & no & no & yes & yes & no \\
\hline P6 & bone & yes & no & yes & no & yes \\
\hline P7 & no & yes & no & no & yes & no \\
\hline P8 & bone & yes & no & yes & yes & no \\
\hline P9 & bone and visceral & no & no & yes & yes & no \\
\hline P10 & bone & yes & no & yes & yes & no \\
\hline P11 & bone & no & no & yes & yes & no \\
\hline $\mathrm{P}_{12}$ & bone & yes & no & yes & yes & no \\
\hline $\mathrm{P}_{13}$ & bone & yes & no & yes & yes & no \\
\hline $\mathrm{P}_{14}$ & bone & yes & no & yes & yes & no \\
\hline P15 & bone & yes & no & yes & yes & yes \\
\hline P16 & bone & yes & no & no & yes & no \\
\hline $\mathrm{P}_{17}$ & bone & yes & no & yes & yes & no \\
\hline P18 & bone & yes & no & yes & no & yes \\
\hline P19 & bone & no & no & yes & yes & no \\
\hline P2O & bone & no & no & yes & yes & yes \\
\hline P21 & bone & yes & no & yes & yes & no \\
\hline P22 & no & no & no & yes & yes & no \\
\hline $\mathrm{P}_{23}$ & bone & no & no & yes & yes & no \\
\hline $\mathrm{P}_{24}$ & bone & no & no & yes & yes & yes \\
\hline $\mathrm{P}_{25}$ & bone & yes & no & yes & yes & no \\
\hline P26 & bone and visceral & no & no & yes & yes & no \\
\hline $\mathrm{P} 27$ & bone and visceral & yes & no & yes & no & yes \\
\hline P28 & bone & no & no & yes & yes & yes \\
\hline P29 & bone and visceral & no & no & yes & yes & yes \\
\hline $\mathrm{P}_{30}$ & bone and visceral & no & no & yes & no & yes \\
\hline$P_{31}$ & bone & no & no & yes & yes & yes \\
\hline $\mathrm{P}_{32}$ & bone & yes & yes & yes & no & yes \\
\hline P33 & visceral & no & no & yes & yes & no \\
\hline P34 & bone & no & yes & yes & yes & no \\
\hline P35 & bone & no & no & yes & yes & yes \\
\hline
\end{tabular}




\begin{tabular}{|c|c|c|c|c|c|c|}
\hline Patient \# & Site of metastases & $\begin{array}{l}\text { Prior } \\
\text { radiation }\end{array}$ & $\begin{array}{l}\text { Prior } \\
\text { surgery }\end{array}$ & $\begin{array}{l}\text { Prior } \\
\text { chemotherapy }\end{array}$ & Abiraterone & Enzalutamide \\
\hline P36 & bone & yes & no & yes & yes & yes \\
\hline P37 & visceral & yes & no & yes & no & yes \\
\hline P38 & bone and visceral & no & no & yes & yes & no \\
\hline P39 & bone and visceral & yes & no & yes & yes & yes \\
\hline $\mathrm{P} 40$ & bone & no & yes & $?$ & $?$ & $?$ \\
\hline $\mathrm{P}_{41}$ & visceral & yes & no & no & no & yes \\
\hline $\mathrm{P} 42$ & bone & yes & no & yes & yes & yes \\
\hline $\mathrm{P} 43$ & bone & yes & no & yes & yes & no \\
\hline P44 & bone & yes & no & yes & yes & no \\
\hline $\mathrm{P} 45$ & bone & no & no & yes & yes & yes \\
\hline \multirow[t]{2}{*}{ Summary } & $\begin{array}{l}88.9 \% \text { bone } \\
\text { metastases }\end{array}$ & $53.3 \%$ yes & $6.7 \%$ yes & $91.1 \%$ yes & $82.2 \%$ yes & $46.7 \%$ yes \\
\hline & $\begin{array}{l}24.4 \% \text { visceral } \\
\text { metastases }\end{array}$ & & & & & \\
\hline
\end{tabular}




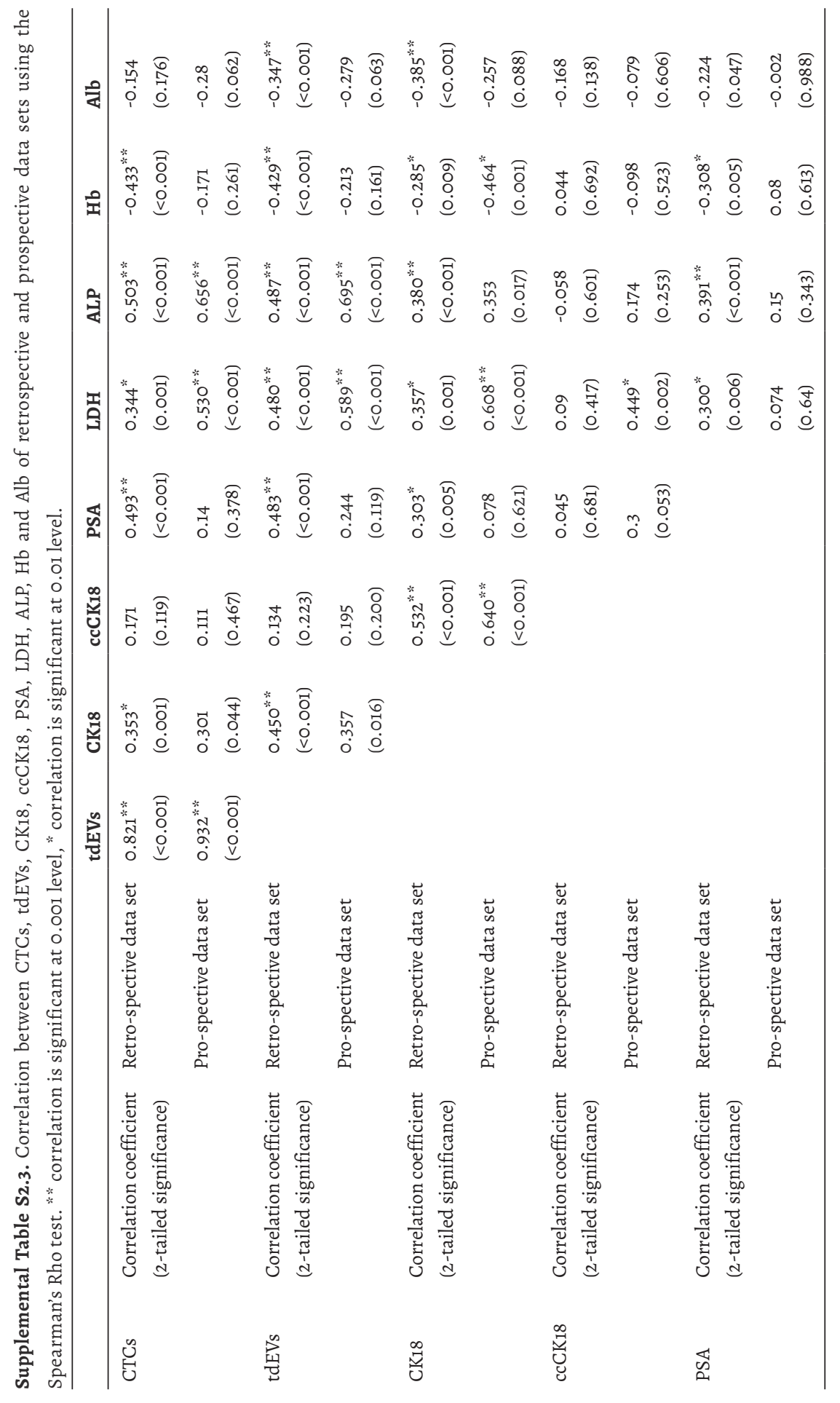




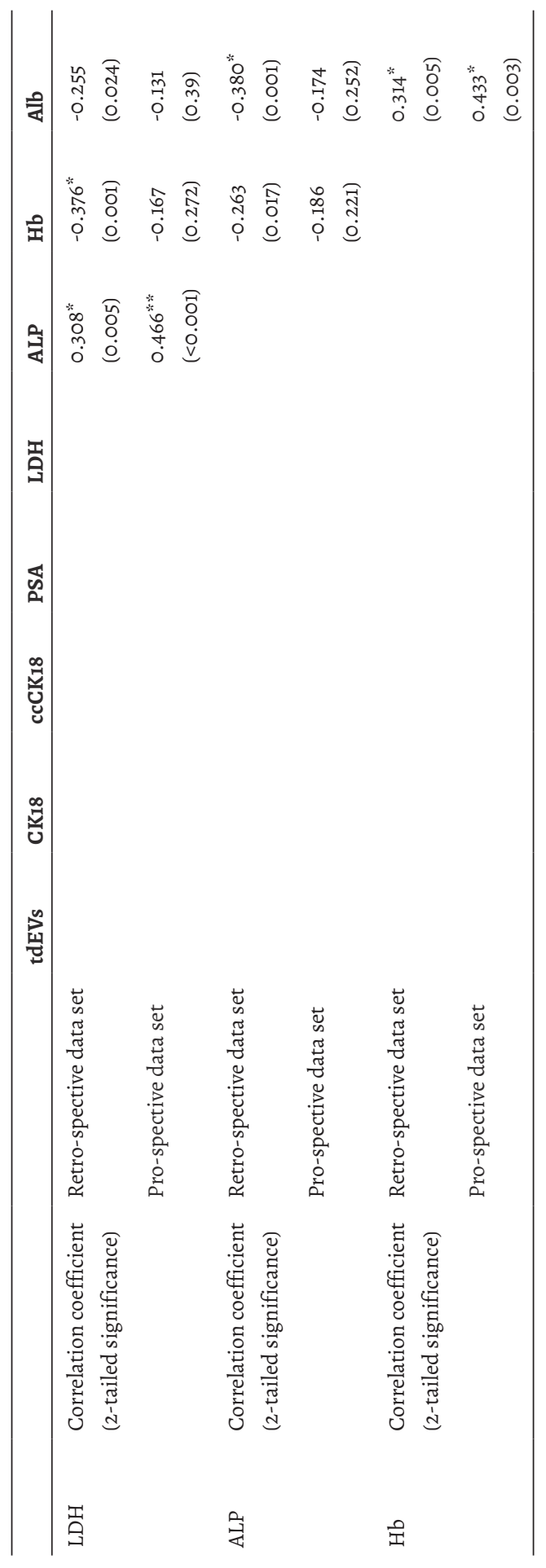


Supplemental Table S2.4.Univariable Cox proportional Hazards regression analysis of age, PSA, ALP, $\mathrm{LDH}, \mathrm{Hb}, \mathrm{Alb}, \mathrm{CK} 18, \mathrm{ccCK} 18$, tdEVs and CTCs for retrospective, prospective and full data sets (Enter method)

\begin{tabular}{|c|c|c|c|c|c|c|}
\hline \multirow[t]{2}{*}{ Variable } & \multirow[t]{2}{*}{ Data set } & \multirow[t]{2}{*}{ Cut-off } & \multirow[t]{2}{*}{ HR } & \multicolumn{2}{|c|}{$95 \% \mathrm{CI}$ for HR } & \multirow[t]{2}{*}{$\mathrm{p}$} \\
\hline & & & & lower & upper & \\
\hline \multirow[t]{3}{*}{ tdEVs } & retrospective & 105 & 3.6 & 2.1 & 6.3 & $<0.001$ \\
\hline & prospective & 105 & 5.0 & 2.2 & 11.1 & $<0.001$ \\
\hline & full & 105 & 3.8 & 2.4 & 5.9 & $<0.001$ \\
\hline \multirow[t]{3}{*}{ CTCs } & retrospective & 5 & 2.6 & 1.5 & 4.3 & $<0.001$ \\
\hline & prospective & 5 & 4.0 & 1.8 & 9.1 & $<0.001$ \\
\hline & full & 5 & 2.8 & 1.8 & 4.4 & $<0.001$ \\
\hline \multirow[t]{3}{*}{ Hemoglobin (Hb) } & retrospective & 12.55 & 0.3 & 0.2 & 0.6 & $<0.001$ \\
\hline & prospective & 12.55 & 0.8 & 0.3 & 2.0 & 0.566 \\
\hline & full & 12.55 & 0.4 & 0.2 & 0.6 & $<0.001$ \\
\hline \multirow[t]{3}{*}{$\mathrm{CK} 18$} & retrospective & 576 & 2.4 & 1.4 & 4.0 & 0.001 \\
\hline & prospective & 232 & 3.5 & 1.6 & 7.8 & 0.001 \\
\hline & full & both & 2.5 & 1.6 & 3.8 & $<0.001$ \\
\hline \multirow[t]{3}{*}{ Prostate specific antigen (PSA) } & retrospective & 87 & 2.3 & 1.4 & 3.8 & 0.002 \\
\hline & prospective & 87 & 1.7 & 0.8 & 3.7 & 0.192 \\
\hline & full & 87 & 2.0 & 1.3 & 3.0 & 0.002 \\
\hline \multirow[t]{3}{*}{ Alkaline phosphatase (ALP) } & retrospective & 126 & 2.2 & 1.3 & 3.6 & 0.004 \\
\hline & prospective & 126 & 2.7 & 1.2 & 6.0 & 0.017 \\
\hline & full & 126 & 2.1 & 1.4 & 3.3 & 0.001 \\
\hline \multirow[t]{3}{*}{ Albumin (Alb) } & retrospective & 3.95 & 0.5 & 0.3 & 0.9 & 0.009 \\
\hline & prospective & 3.95 & 0.8 & 0.3 & 2.122 & 0.658 \\
\hline & full & 3.95 & 0.6 & 0.3 & 0.9 & 0.010 \\
\hline \multirow[t]{3}{*}{ Lactate dehydrogenase (LDH) } & retrospective & 230 & 1.9 & 1.1 & 3.1 & 0.015 \\
\hline & prospective & 230 & 2.7 & 1.2 & 5.8 & 0.014 \\
\hline & full & 230 & 2.0 & 1.3 & 3.0 & 0.002 \\
\hline \multirow[t]{3}{*}{ Age (per 10 years) } & retrospective & & 1.2 & 0.9 & 1.6 & 0.407 \\
\hline & prospective & & 1.0 & 0.6 & 1.6 & 0.889 \\
\hline & full & & 1.1 & 0.9 & 1.4 & 0.484 \\
\hline \multirow[t]{3}{*}{ ccCK18 } & retrospective & 265 & 1.2 & 0.7 & 2.0 & 0.483 \\
\hline & prospective & 81 & 1.3 & 0.6 & 2.8 & 0.484 \\
\hline & full & both & 1.2 & 0.8 & 1.8 & 0.460 \\
\hline
\end{tabular}





\title{
Chapter 3
}

\section{Tumor-derived extracellular vesicles in blood of metastatic cancer patients associate with overall survival}

\author{
Afroditi Nanou, M. Craig Miller, Leonie L. Zeune, Sanne de Wit, Cornelis J.A. Punt,
} Harry J.M. Groen, Daniel F. Hayes, Johann S. de Bono, Leon W.M.M. Terstappen

British Journal of Cancer 2020

\begin{abstract}
Circulating tumor cells (CTCs) in blood associate with Overall Survival (OS) of cancer patients, but they are detected in extremely low numbers. Large tumor-derived extracellular vesicles (tdEVs) in castration-resistant prostate cancer (CRPC) patients are present at around 20 times higher frequencies than CTCs and have equivalent prognostic power. In this study, we explored the presence of tdEVs in other cancers and their association with OS. The open-source ACCEPT software was used to automatically enumerate tdEVs in digitally stored Cellsearch ${ }^{\circledR}$ images obtained from previously reported CTC studies evaluating OS in 190 CRPC, 450 metastatic colorectal cancer (mCRC), 179 metastatic breast cancer (MBC) and 137 non-small cell lung cancer (NSCLC) patients before the initiation of a new treatment. Presence of unfavorable CTCs and tdEVs is predictive of OS, with respective Hazard Ratios (HRs) of 2.4 and 2.2 in CRPC; 2.7 and 2.2 in MBC; 2.3 and 1.9 in mCRC; and 2.0 and 2.4 in NSCLC. tdEVs have equivalent prognostic value as CTCs in the investigated metastatic cancers. CRPC, mCRC, and MBC (but not NSCLC) patients with favourable CTC counts can be further prognostically stratified using tdEVs. Our data suggests that tdEVs could be used in clinical decision making.
\end{abstract}




\subsection{Introduction}

Characterization of a patient's tumor is frequently assessed on the primary tumor. However, it is well recognized that the phenotype of a tumor is highly heterogeneous; hence, a biopsy in a selective area is very restrictive in representing the whole tumor ${ }^{1,2}$. Moreover, the lesions in metastatic sites further complicate the tumor pattern as they can have substantially different features than the primary tumor ${ }^{3-5}$. Additionally, tumors evolve over time and treatments while developing drug resistance mechanisms ${ }^{6}$. These observations emerge the importance of a biopsy that could provide clinicians with real-time data to facilitate treatment-decision making. In theory, subsequent collections of solid biopsies from multiple sites could be a solution; however, in practice, not all sites are accessible by surgery and such a procedure is highly invasive leading to patient discomfort and health complications. On the other hand, liquid biopsies require a minimally invasive biofluid sampling; consequently, they can be performed in short time intervals providing clinicians with a real-time snapshot of the disease ${ }^{7}$. Importantly, the detected tumor material in biofluids, namely circulating tumor cells (CTCs), tumor-derived extracellular vesicles (tdEVs) and circulating tumor DNA (ctDNA), is clinically relevant and can better reflect the characteristics of the metastatic sites ${ }^{8}$.

Specifically, the field of tdEVs has gained a lot of attention during the last few years, mainly because of their increased prevalence and promise as potential biomarkers to aid in the disease management of cancer patients ${ }^{9-15}$. The reported size range of tdEVs varies between $30-10,000 \mathrm{~nm}$, with the diameter of exosomes being 30 to $300 \mathrm{~nm}$, of microvesicles $<1,000 \mathrm{~nm}$ and of large oncosomes between 1,000 and 10,000 $\mathrm{nm}^{16-18}$. All different tdEV subclasses have been reported to play various roles directly related to the disease progression and metastatic processes ${ }^{19-25}$. However, the isolation, enumeration, differentiation and molecular profiling of pure tdEVs from the blood of cancer patients is challenging because of all the contaminants present, including proteins, protein aggregates, free nucleic acids (RNA and DNA), platelets and EVs of different cell origins ${ }^{26,27}$. Hence, enrichment and/or depletion techniques are necessary for the specific isolation of tdEVs and their downstream characterization.

Previously, our group reported the presence of EpCAM+, CK+, DNA-, CD45- "tumor microparticles" and "CTC fragments" that were considered by-products of cancer apoptosis ${ }^{28,29}$. These particles have relatively large sizes $(>1 \mu \mathrm{m})$ and can be isolated together with CTCs from $7.5 \mathrm{~mL}$ of peripheral blood after immunomagnetic selection targeting the epithelial cell adhesion molecule (EpCAM) expressed on their surface membrane. We used the term tumor-derived EVs (tdEVs) to describe these objects. The isolation and labeling of the EpCAM enriched sample is performed using the FDA-cleared CellSearch ${ }^{\circledR}$ system. For the automated enumeration of tdEVs, the open- 
source ACCEPT software (http://github.com/LeonieZ/ACCEPT) was used. ACCEPT segments all detected objects in the fluorescence images and measures 10 parameters in each fluorescence channel regarding their morphology and fluorescence intensity. By defining and applying linear gates, different classes of objects (leukocytes, CTCs, tdEVs) found in the fluorescence CellSearch images can be enumerated in an automated manner. Following that approach, we previously reported that these large tdEVs are present in the blood of castration-resistant prostate cancer (CRPC) patients in approximately 20 times higher frequencies compared to circulating tumor cells (CTCs) and have a significant association with poor prognosis ${ }^{14}$. Here, we explore whether the presence of EpCAM+, CK+, DNA-, CD45-tdEVs in metastatic breast cancer $(\mathrm{MBC})$, metastatic colorectal cancer (mCRC), and non-small cell lung cancer (NSCLC) patients is associated with overall survival (OS) and we determine whether or not tdEVs can further improve prognostication of cancer patients.

\subsection{Materials and Methods}

\subsubsection{Patient samples}

190 CRPC (IMMC38 clinical trial, NCTO0133900) ${ }^{30}, 450$ mCRC (CAIRO II clinical trial, NCT00208546) ${ }^{31,32}$, 179 MBC (IMMCo1 clinical trial) ${ }^{33}$, and 137 NSCLC patient samples ${ }^{34-36}$, along with 93 healthy control samples (IMMCo6 clinical trial, NCToo133913) ${ }^{37}$, were included. The included samples corresponded to patients before the initiation of a new treatment. Patient characteristics are provided in Table 3.1. All individuals provided written informed consent prior to participation in the trial protocols approved by institutional review boards at the participating centers of the studies ${ }^{31-38}$.

Table 3.1. Patient characteristics

\begin{tabular}{lllll}
\hline $\begin{array}{l}\text { Metastatic cancer patients } \\
\text { Number of patients }\end{array}$ & $\begin{array}{l}\text { CRPC } \\
\mathbf{1 9 0}\end{array}$ & $\begin{array}{l}\text { MBC } \\
\mathbf{1 7 9}\end{array}$ & $\begin{array}{l}\text { MCRC } \\
\mathbf{4 5 0}\end{array}$ & $\begin{array}{l}\text { NSCLC } \\
\mathbf{1 3 7}\end{array}$ \\
\hline Age (years) & & & \\
\hline Median (range) & $70(49-92)$ & $59(27-86)$ & $63(27-83)$ & $65(29-83)$ \\
\hline Gender & & & $271(60 \%)$ & $74(54 \%)$ \\
Male & $190(100 \%)$ & $0(0 \%)$ & $179(40 \%)$ & $63(46 \%)$ \\
Female & $0(0 \%)$ & $179(100 \%)$ & $0(0 \%)$ \\
unknown & $0(0 \%)$ & $0(0 \%)$ & $0(0 \%)$ & $78(57 \%)$ \\
\hline ECOG Performance Status & & & $286(64 \%)$ & $49(36 \%)$ \\
\hline & $87(46 \%)$ & $83(46 \%)$ & $153(34 \%)$ & $5(4 \%)$ \\
1 & $80(42 \%)$ & $73(41 \%)$ & $2(0 \%)$ & \\
2 & $17(9 \%)$ & $17(10 \%)$ & & $5(4 \%)$ \\
3 & $0(0 \%)$ & $1(1 \%)$ & & \\
4 & $0(0 \%)$ & $0(0 \%)$ & $5(2 \%)$ & \\
\hline unknown & $6(3 \%)$ & $5(3 \%)$ & & \\
\hline
\end{tabular}




\begin{tabular}{|c|c|c|c|c|}
\hline \multicolumn{5}{|l|}{ Line of therapy } \\
\hline 1 & $132(70 \%)$ & $75(42 \%)$ & $450(100 \%)$ & $36(26 \%)$ \\
\hline 2 & $29(15 \%)$ & $27(15 \%)$ & & $21(15 \%)$ \\
\hline $3+$ & $29(15 \%)$ & $75(42 \%)$ & & $80(59 \%)$ \\
\hline unknown & & $2(1 \%)$ & & \\
\hline \multicolumn{5}{|c|}{ Therapy type started after blood draw } \\
\hline Chemo- & $11(6 \%)$ & $75(42 \%)$ & & $32(23 \%)$ \\
\hline Hormone & & $45(25 \%)$ & & \\
\hline Molecular & & $8(4 \%)$ & & $20(15 \%)$ \\
\hline Immuno- & & & & $80(58 \%)$ \\
\hline Other & & $1(1 \%)$ & & \\
\hline Chemo-/Hormone & $126(66 \%)$ & $11(6 \%)$ & & \\
\hline Chemo-/Molecular & & $23(13 \%)$ & $450(100 \%)$ & \\
\hline Chemo-/Other & $7(4 \%)$ & $1(1 \%)$ & & \\
\hline Hormone/Molecular & & $8(4 \%)$ & & \\
\hline Chemo-/Molecular/Hormone & & $2(1 \%)$ & & \\
\hline Chemo-/Hormone/Other & $45(24 \%)$ & & & \\
\hline Unknown & $1(1 \%)$ & $5(3 \%)$ & & $5(4 \%)$ \\
\hline \multicolumn{5}{|c|}{ Follow-up time (in months) Median (min-max) } \\
\hline Alive & $\begin{array}{l}30.4 \\
(1.9-39.0)\end{array}$ & $\begin{array}{l}20.6 \\
(1.3-48.8)\end{array}$ & $\begin{array}{l}20.2 \\
(0.0-34.0)\end{array}$ & $\begin{array}{l}8.8 \\
(0.7-30.1)\end{array}$ \\
\hline Dead & $\begin{array}{l}11.6 \\
(0.7-39.3) \\
\end{array}$ & $\begin{array}{l}9.9 \\
(0.4-31.7) \\
\end{array}$ & $\begin{array}{l}12.2 \\
(0.4-32.3)\end{array}$ & $\begin{array}{l}4.6 \\
(0.7-25.3) \\
\end{array}$ \\
\hline \multicolumn{5}{|l|}{ Status at last follow-up } \\
\hline Alive & $53(28 \%)$ & $77(43 \%)$ & $194(43 \%)$ & $68(50 \%)$ \\
\hline Dead & $137(72 \%)$ & $102(57 \%)$ & $256(57 \%)$ & $69(50 \%)$ \\
\hline
\end{tabular}

\subsubsection{Isolation and identification of CTCs and tdEVs}

Digitally stored CellSearch ${ }^{\circledR}$ (Menarini Silicon Biosystems, Huntingdon Valley PA, USA) image files from the abovementioned CTC studies were re-analysed. Briefly, CTCs and tdEVs were immunomagnetically isolated from $7.5 \mathrm{~mL}$ of peripheral blood collected in Cell Save tubes using the CellSearch system. The EpCAM enriched cells were stained with the nucleic acid dye DAPI and the staining reagent of the CTC kit including mouse monoclonal antibodies against CD45 (clone HI30) conjugated to APC and mouse monoclonal antibodies against Cytokeratins 8, 18 and 19 (CK) (clones $\mathrm{C} 11$ and $\mathrm{A} 53-\mathrm{B} / \mathrm{A2}$ ) conjugated to $\mathrm{PE}$. In case of CRPC, MBC and $\mathrm{MCRC}$, no extra markers were used in the FITC and PerCP channels. In case of NSCLC, the EPCAM enriched cells had been additionally labeled with mouse monoclonal antibodies against CD16 (clone 3G8) conjugated to PerCP (Marker 2) and in some of the cases with the wheat germ agglutinin conjugated to Alexa 488 (Marker 1) or with mouse 
monoclonal antibodies against CK 1-8, 10, 14, 15, 16, 19, 20 (clones LP5K, Ks20.10, and $\mathrm{AE} 1 / \mathrm{AE} 4$ ) conjugated to FITC (Marker 1) to address research points of previously reported studies. The subsets of patient samples with additional Marker 1 or/and 2 labeling can be found in Supplementary Table S3.1. The immunofluorescently stained suspension was placed in a cartridge contained within a Magnest ${ }^{\circledR}$ as previously described ${ }^{37}$. The image acquisition was performed on the Cellspotter ${ }^{\mathrm{TM}}$ Analyzer for the older IMMCo1 study (MBC) and the healthy donors included in the IMMC06 study. The image acquisition of the IMMC38 (CRPC), CAIRO II (MCRC) and NSCLC studies was performed on the CellTracks ${ }^{\circledR}$ Analyzer II $^{\mathrm{TM}}$. Both systems are semi-automated fluorescence microscopes equipped with computer controlled X, Y, Z stages, a NA 0.45 10x objective, a Mercury Arc lamp, a 12-bit CCD camera and filter cubes for DAPI, PE, APC and FITC. Typically, 175 images per channel are taken to cover the entire surface of the cartridge ${ }^{39}$.

\subsubsection{CTC counts and automated enumeration of tdEVs with ACCEPT}

To obtain accurate counts of CTCs and tdEVs, the manual CTC counts were extracted from the CellTracks Analyzer II. For tdEV enumeration, the digitally stored fluorescence image files were re-analyzed with the open-source ACCEPT software v1.1 (http://github.com/LeonieZ/ACCEPT) using the "Full Detection" function. After that analysis, 10 morphological and fluorescence signal intensity measurements, for each object found in the images, are extracted per channel. These measurements can be used to design linear gates to identify different classes of objects in the images ${ }^{14,34}$. The definition of tdEVs in case of CRPC, MBC, mCRC was EpCAM+, CK+, DAPI-, CD45-particles of a diameter $<14 \mu \mathrm{m}$. The background PE fluorescence in the images acquired on the Cellspotter Analyzer was higher compared to the CellTracks Analyzer II, resulting in the use of a different tdEV gate for each platform. The applied tdEV gate for images acquired on CellTracks Analyzer II was: Mean Intensity CD $45 \leq 5$, Mean Intensity DNA $\leq 5$, Mean Intensity $C K>60$, Mean Intensity Marker $1 \leq 5$, Mean Intensity Marker $2 \leq 5$, Max Intensity $C K>90$, Size $C K \leq 150 \mu \mathrm{m}^{2}$, Perimeter $C K>5$ pixels, Eccentricity $\mathrm{CK} \leq 0.8$, and Perimeter to Area $C K \leq 1^{14}$. For the images acquired on the Cellspotter, the setting of Standard Deviation for CK > 40 was implemented in the tdEV gate instead of the CK Max Intensity. In case of NSCLC, tdEVs should be additionaly CD16- (an already included criterion in the aforementioned tdEV gate as Mean Intensity Marker $2 \leq 5)$. However, tdEVs could be either positive or negative for Marker 1 (wheat germ agglutinin or CK 1-8, 10, 14, 15, 16, 19, 20). In order to be able to compare among different NSCLC patient samples, we did not include any requirements for the Mean Intensity Marker 1.

\subsubsection{Statistical analysis}

Statistical analysis was performed using SPSS 23.0 (SPSS Inc., Chicago, IL, USA). For each cancer type, a two-tailed Spearman's Rho test was performed to evaluate the 
relation between the CTC and tdEV counts. The non-parametric Wilcoxon Signed Ranks test was used to test the equality of the distributions for the matched CTC and tdEV counts within each cancer type. The non-parametric Mann-Whitney U test was used to test the equality of the distributions for the CTC and tdEV counts in the healthy donors compared to each patient cohort as well as all patients together. Overall Survival (OS) for each patient was defined as the elapsed time in months between the baseline blood draw date and the date of death or the date of last follow-up. Patients alive at the end of the study or lost during the follow-up period were censored. Median OS was estimated by Kaplan Meier survival curves and survival curves for favorable and unfavorable groups based on CTC and/or tdEV counts were compared using the non-parametric log-rank test. Cox proportional-hazards regression analysis was used to determine the univariable hazard ratios (HRs) for OS with $95 \%$ confidence intervals (CIs). A final multivariable Cox model for each cancer type was fit including the significant variables from the univariable Cox proportional hazards regression analysis. Due to correlation between some of the included variables, the final model was selected using forward stepwise elimination $\left(\mathrm{p}_{\text {in }}=0.05\right.$ and $\left.\mathrm{p}_{\text {out }}=0.10\right)$. The opensource web application Cutoff Finder (http://molpath.charite.de/cutoff) was used to calculate the HRs for OS with $95 \%$ CIs over a wide range of cut-off values for the CTC and tdEV counts for the full data sets as well as for tdEV counts in the subset of patients with favorable CTC counts. The optimal cut-off values of tdEVs for patients with favorable CTCs were defined as the points with the most significant split (logrank test). Cutoff Finder uses the $\mathrm{R}$ code to provide optimization and visualization tools for cut-off determination ${ }^{40}$. Receiver Operating Characteristic (ROC) Curves were used for each cancer type to assess the performance of CTCs and tdEVs in classifying patients based on shorter than median OS or death.

\subsection{Results}

\subsubsection{ACCEPT display of CTCs and tdEVs}

All objects found in the fluorescence images are visualized using the quantitative display of the ACCEPT toolbox. Figure 3.1 shows examples of manually scored CTCs and automatically scored tdEVs found in the Cellsearch images.

\subsubsection{CTC and tdEV frequencies in CRPC, MBC, mCRC and NSCLC}

The frequencies of EPCAM+ CTCs and tdEVs identified in blood samples obtained from CRPC, MBC, MCRC and NSCLC patients are provided in Figure 3.2. Samples from 93 healthy donors were included as a reference. The CTC distribution of healthy donors was highly significantly different compared to the respective distribution of CRPC, MBC and NSCLC ( $p<0.001$, Mann-Whitney U test) but not mCRC ( $p>0.05$, Mann-Whitney U test). In case of tdEVs, the distribution of healthy donors was highly significantly 


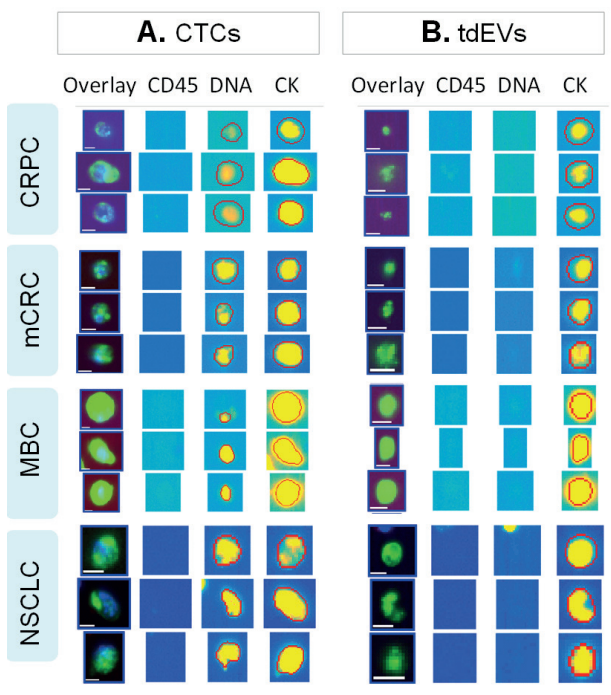

Figure 3.1. Examples of objects manually classified as CTCs by individual users (Panel A) or automatically classified as tdEVs using the open-source ACCEPT software (Panel B) on images obtained from CRPC, MBC, mCRC and NSCLC cancer patients. The red contours around the objects indicate the contours as detected by the ACCEPT image analysis algorithm. All objects were isolated from $7.5 \mathrm{~mL}$ of blood using the CellSearch system. Scale bars indicate $6.4 \mu \mathrm{m}$.
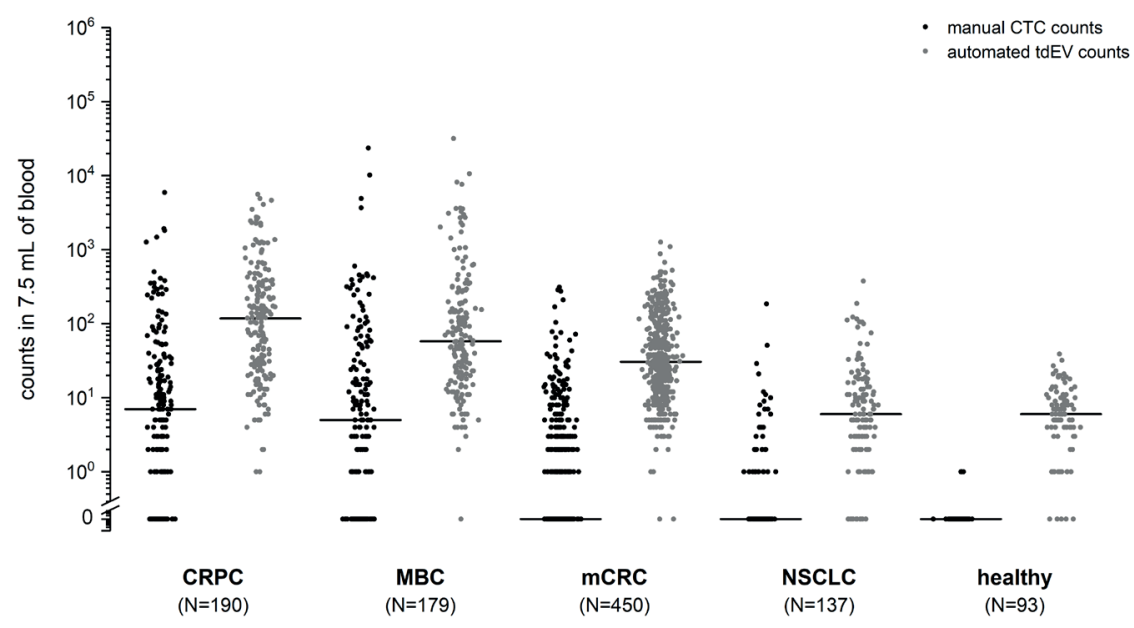

Figure 3.2. Dot plots of manual CTC counts (in black) and automated tdEV counts (in gray) in the blood of individuals with different cancer types. Horizontal black lines indicate median values. tdEV counts are significantly higher compared to the respective CTC counts in CRPC, MBC, MCRC and NSCLC cancer patients ( $\mathrm{p}<0.05$ for all 4 comparisons, Wilcoxon Signed Ranks test). CTC and tdEV counts of 93 healthy donors were used as a reference. 
different compared to the respective distribution of CRPC, MBC and $\mathrm{MCRC}$, but not NSCLC. Comparison of the pooled patient CTC and tdEV dataset to the reference dataset of healthy donors resulted in a highly significantly different distribution of only tdEVs. Notably, the median and average tdEV counts are an order of magnitude higher compared to the respective CTC counts $(p<0.01$, Wilcoxon Signed Ranks test), with 96.4\% of all patients (186/190 CRPC, 169/179 MBC, 448/450 mCRC and 119/137 NSCLC patients) having higher tdEV counts. CRPC patients had the highest median CTC and tdEV counts, followed by MBC, MCRC and NSCLC patients. CTC and tdEV counts were significantly correlated in all cancer types, as illustrated in Supplementary Figure S3.1. The correlation coefficient between CTC and tdEV counts in these cancer types were tested using Spearman's Rho test and was found to be 0.87 for CRPC ( $p<0.001), 0.70$ for $\mathrm{MBC}$ and $\mathrm{mCRC}(p<0.001)$, and 0.44 for NSCLC cancer $(p<0.001)$.

Due to skewed distribution, we chose to use the median value plus 2 standard deviations (SD) of tdEV counts detected in the 93 healthy donors as a normal reference range. This resulted in a normal range of 0 to $20 \mathrm{tdEVs}$ per $7.5 \mathrm{~mL}$ of blood.

\subsubsection{Association of CTCs and tdEVs with OS in cancer patients}

The association of CTCs and tdEVs in CRPC, MBC, mCRC and NSCLC with OS was evaluated using Kaplan Meier (KM) plots (Figure 3.3). For CTCs, the same cut-offs established in the original studies ( 5 for CRPC and MBC and 3 for $\mathrm{MCRC}$ ) were used to dichotomize patients into favorable and unfavorable CTC groups. For NSCLC patients, 1 CTC was used as a cut-off. For tdEVs, the cut-off value used to dichotomize patients into groups with favorable and unfavorable tdEVs was defined as the upper bound of the normal reference range ( $\geq 20 \mathrm{tdEVs}$ ). Univariable Cox proportional-hazards regression analysis was used to estimate the HR for OS between the favorable and unfavorable CTC and tdEV groups. As shown in Figure 3.3, cancer patients with $\geq 20 \mathrm{tdEVs} / 7.5 \mathrm{~mL}$ have an approximately 2 -fold higher risk of death compared to patients with $<20 \mathrm{tdEVs}$ ( $\mathrm{p}<0.05$, log-rank test). The stratification of the same patients based on their CTC counts resulted in slightly higher HRs for OS in all cancer types, except for NSCLC, where the HR was slightly higher for tdEVs ( $\mathrm{p}<0.05$, log-rank test, Figure 3.3).

To demonstrate the association between increasing CTC and tdEV load and OS, KM plots were generated. For this analysis, CTCs and tdEVs for each cancer type were binned into four groups, except for the CTC analysis of mCRC and NSCLC, where the CTC counts were binned into only three groups because the majority of patients did not have CTCs. As shown in Figure 3.4, increasing tdEV load in the blood of cancer patients (Panel B) is significantly associated with worsening OS. The same pattern can be seen for CTCs (Panel A), although the narrower range of CTC values leads to more unequal numbers of patients in each risk group compared to their stratification based on tdEV values. 


\section{A. CTCs}
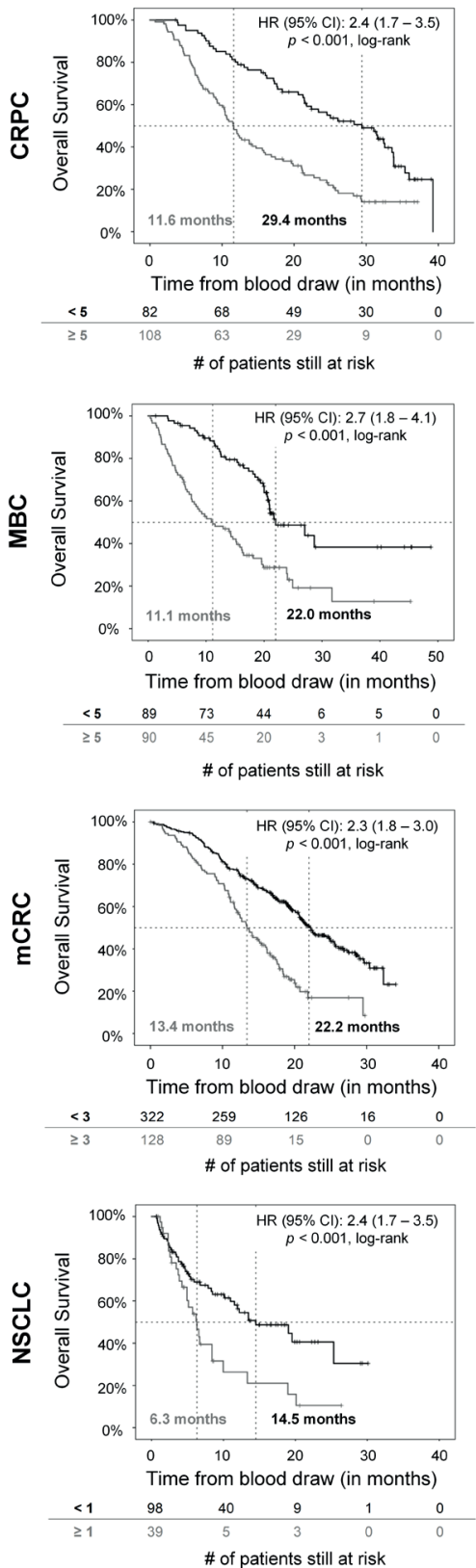

\section{B. tdEVs}
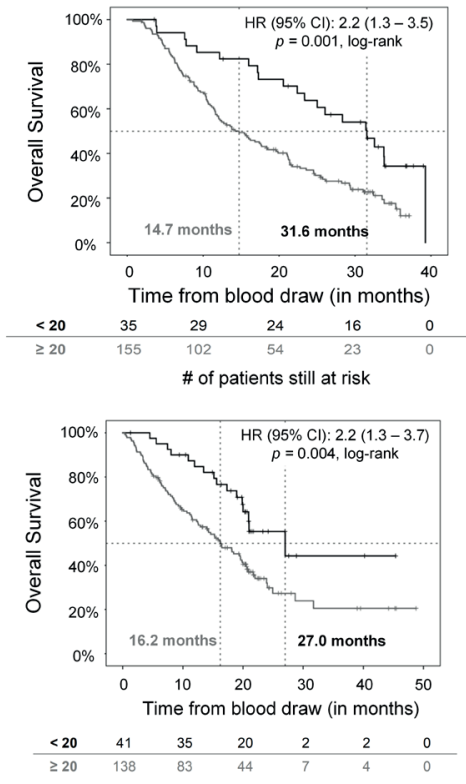

\# of patients still at risk
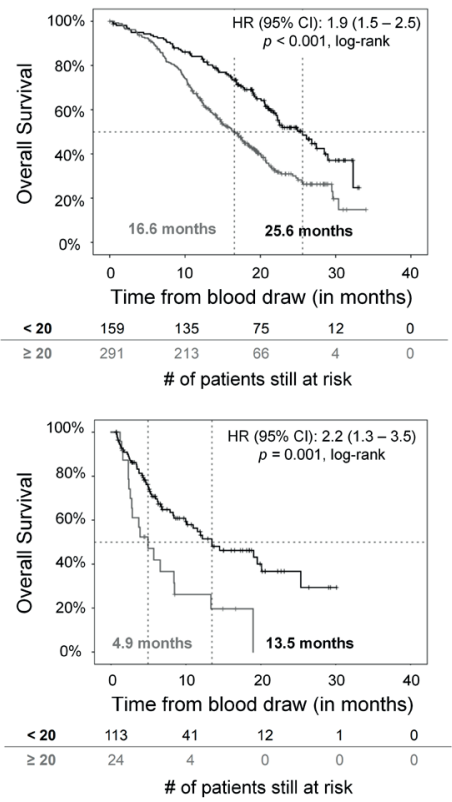

Figure 3.3. Kaplan Meier plots for overall survival in CRPC, MBC, MCRC and NSCLC patients with favorable and unfavorable CTC (Panel A) and tdEV (Panel B) counts using different cut-off values for CTCs ( 5 for CRPC and MBC, 3 for $\mathrm{MCRC}$ and 1 for NSCLC) and the same cut-off value of 20 for tdEVs. 


\section{A. CTCs}
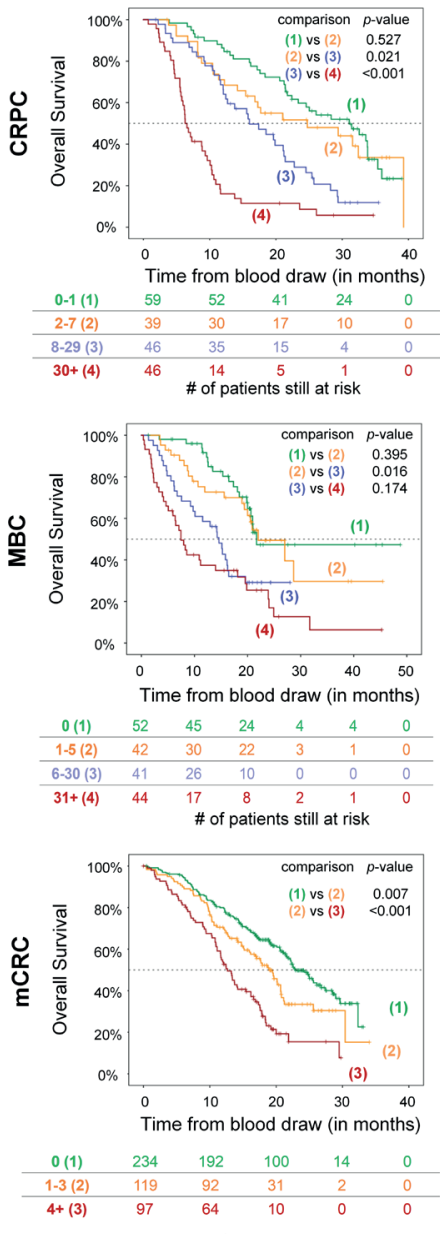

\# of patients still at risk
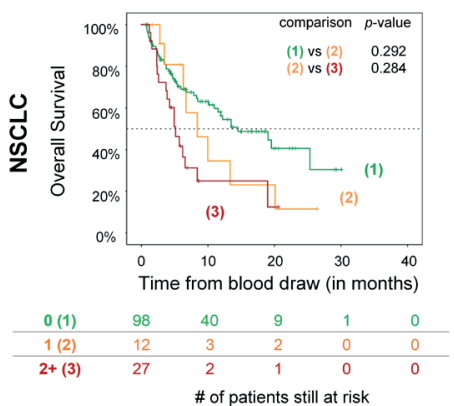

\section{B. tdEVs}
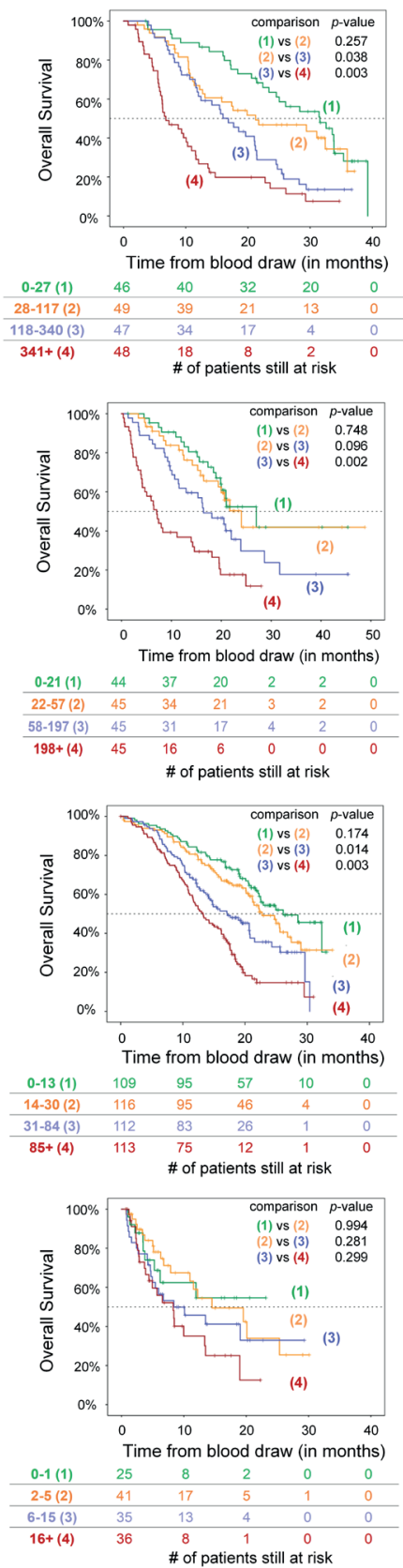

Figure 3.4. Kaplan Meier plots for overall survival in CRPC, MBC, MCRC and NSCLC patients with increasing levels of CTCs (Panel A) and tdEVs (Panel B). Increasing tdEV counts are associated with worse OS in all cancer types, similarly to CTCs. 
To investigate the dependence of $\mathrm{HR}$ for all four different cancer types on different cut-off values for both CTCs and tdEVs, overview plots of HR (including 95\% CIs) for OS were generated by the open-source Cutoff Finder web application (Supplementary Figure S3.2). For CRPC, MBC and mCRC cancer, more than $94 \%$ of all possible cut-off values for both CTCs and tdEVs lead to a significant dichotomization of patients for OS. For NSCLC, all of the possible CTC cut-off values and $67 \%$ of possible tdEV thresholds resulted in patient stratification with a significant HR. Based on the same overview plots, it is clear that HRs for both CTCs and tdEVs in all different cancer types are similar.

The overlap of ROC curves that were generated to evaluate the performance of CTCs and tdEVs in classifying patients based on their OS and experiencing death (Supplementary Figure S3.3), further supports the finding that CTCs and tdEVs are equivalent prognostic biomarkers of OS.

\subsubsection{Stratification of patients with favorable CTC counts based on tdEVs}

To evaluate whether patients with favorable CTC counts could be further stratified using tdEVs, the Cutoff Finder web application was used to plot the resulting HRs (with $95 \% \mathrm{CI}$ ) against the different cut-off values of tdEVs for each cancer type (Figure 3.5A-C). For CRPC, the optimal tdEV cut-off (i.e. the cut-off leading to the highest HR with a p-value $<0.001$, log-rank test) was $\geq 89$, with $13 \%$ of the patients with favorable CTC counts having elevated (unfavorable) tdEV counts. For MBC, the optimal tdEV cut-off was $\geq 80$, with $11 \%$ of the patients with favorable CTC counts having elevated tdEVs. For mCRC, the optimal tdEV cut-off was $\geq 40$, with $24 \%$ of the patients with favorable CTC counts having unfavorable tdEV counts. For NSCLC, no cut-off value for tdEVs was found that led to a significant dichotomization of patients with favorable CTC counts. KM plots were generated for patients with favorable CTC counts based on the aforementioned optimal tdEV cut-off values for each cancer (Figure 3.5D-F). Patients with unfavorable tdEV counts had significantly worse OS in comparison to patients with favorable tdEV counts, with a 4.7-fold higher risk of death (95\% CI: 2.2 10.3) in CRPC; a 4.9-fold higher risk (95\% CI: 2.2-11.2) in MBC, and a 2-fold higher risk (95\% CI: $1.5-2.9)$ in $\mathrm{mCRC}$.

\subsubsection{Univariable and multivariable associations between potential risk factors and OS of CRPC, MBC, MCRC and NSCLC patients}

Different variables for each cancer type were evaluated as potential risk factors of OS using univariable cox proportional hazards regression analysis (Supplementary Table S3.2). For the multivariable analysis, the significant predictors of the univariable analysis were included (Supplementary Table S3.3).

In case of CRPC, the tested variables were age, ECOG status, haemoglobin and the log transformed CTCs, tdEVs, PSA, LDH, ALP, albumin and testosterone. All variables, except for testosterone, were significant and used as input variables in 
the multivariable regression analysis. The final multivariable model included ECOG status, age, haemoglobin, tdEVs and LDH.

In case of MBC, ECOG status, age, ER, HER2 and PR status of the tumor, time to metastasis, number of metastatic sites, line of therapy, type of therapy, log transformed CTCs and tdEVs were evaluated as potential risk factors. ECOG status, PR status, number of metastatic sites, type of therapy, CTCs and tdEVs were significant and used as input variables in the multivariable regression analysis. The final multivariable model included the number of metastatic sites, ECOG status and CTCs.

In case of mCRC, the tested variables were the treatment arm, ECOG status, age, LDH (normal versus abnormal), gender, prior adjuvant therapy, more than 1 affected organs and the log transformed CTCs and tdEVs. All variables apart from the treatment arm, the prior adjuvant therapy and the gender were significant predictors of OS and were used as input variables in the multivariable regression analysis. The final multivariable model included the ECOG status, age and both CTCs and tdEVs.

In case of NSCLC, the tested variables were the ECOG status, type of treatment, age, gender and the log transformed CTCs and tdEVs. Except for the type of treatment, all variables were significant predictors of OS and used as input variables in the multivariable regression analysis. The final multivariable model included the ECOG status, age and CTCs.

\subsection{Discussion}

The FDA-cleared CellSearch ${ }^{\circledR}$ system presents thumbnail images containing CK-PE and DAPI signals to the operator for manual classification of CTCs ${ }^{39}$. The operator bias in CTC classification can be improved by providing quantitative information of the objects in the thumbnails and can be eliminated by the use of gates with the open-source ACCEPT software (http://github.com/LeonieZ/ACCEPT) ${ }^{14,41-43}$. Further improvements in the classification of CTCs can be obtained through the use of deep learning ${ }^{44}$; however, for its implementation on larger datasets, significant improvements in the software are still needed. Therefore, in this study, we elected to use the original CTC scores of the studies. The introduced operator bias together with the assay variations and the low cut-off values of 1, 3 and 5 CTCs per $7.5 \mathrm{~mL}$ of blood, make an accurate assessment impossible. As an example, two separate tubes of blood drawn from the same patient at the same time could very likely show that one tube had 4 CTCs, while the other tube had 6 CTCs, resulting in the assignment of the patient into a different risk group ${ }^{45}$. 
A
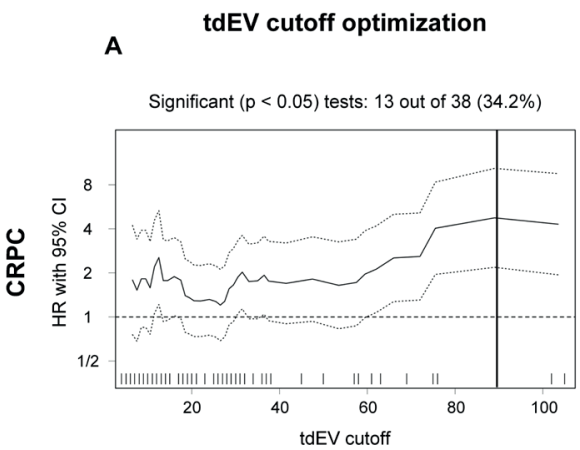

B

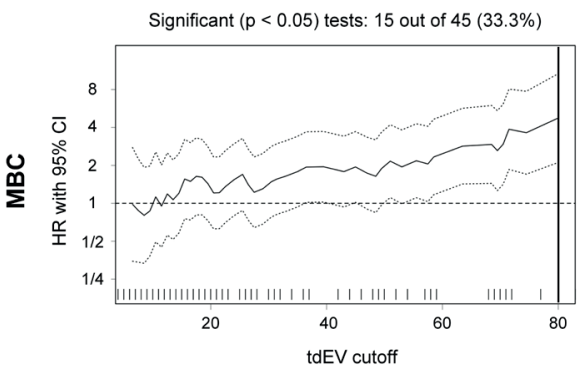

C

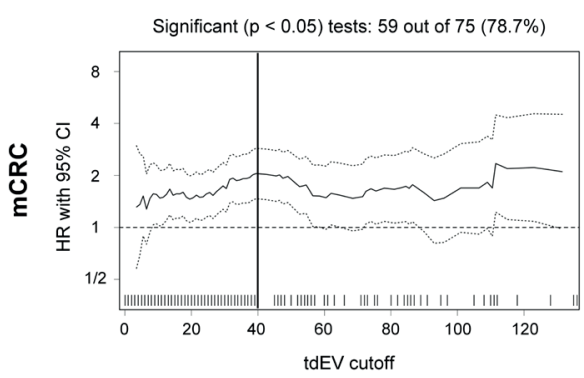

\section{Kaplan Meier plots of OS}

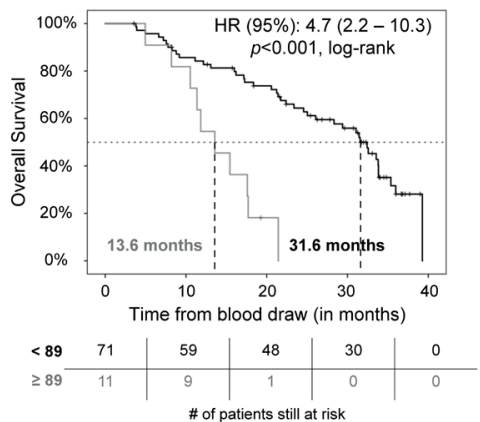

E

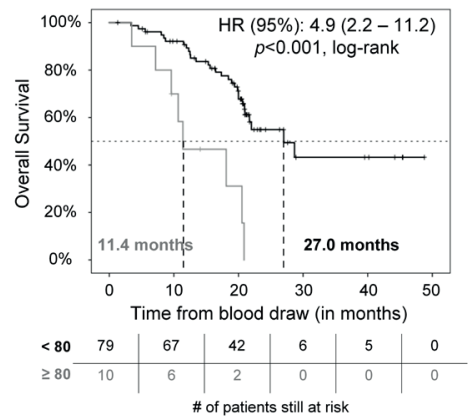

$\mathbf{F}$

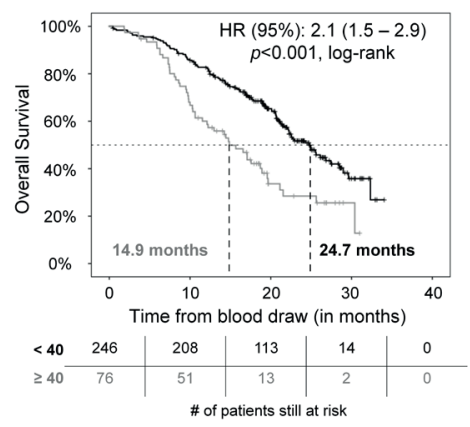

Figure 3.5. Overview plots of HRs (with $95 \% \mathrm{CIs}$ ) for all possible cut-off values for tdEV counts in CRPC (Panel A), MBC (Panel B) and MCRC (Panel C) patients with favorable CTC counts ( $<5$ for CRPC and $M B C$ and $<3$ for $\mathrm{mCRC}$ ), as generated by the Cutoff Finder software. The optimal cut-offs, determined as the point that resulted in the most significant split (log-rank test), are indicated by a vertical line in each plot. Kaplan Meier plots for overall survival in CRPC (Panel D), MBC (Panel E), and mCRC (Panel F) patients with favorable CTC counts by farovable and unfavorable tdEV counts were generated based on the optimal tdEV cut-off values. Favorable CTC patients with unfavorable (elevated) tdEV counts had significantly worse OS compared to favorable CTC patients with favorable (below the selected cut-off values) tdEV counts. 
For tdEVs, no manual scores can be obtained as the CellSearch generated thumbnails do not include objects with only CK-PE. Whether or not deep learning can be also used to improve detection and classification of tdEVs remains, as of yet, unanswered. Notably, ACCEPT tdEV counts are found in at least one order of magnitude higher frequencies compared to CTCs in all different cancer types, thereby confirming our previous findings in CRPC ${ }^{14}$. The high degree of correlation between CTCs with tdEVs that we found in CRPC, MBC and mCRC (Supplementary Figure S3.1) can likely be attributed to a similar shedding of tdEVs from the primary and metastatic sites as with the CTCs. Origin of tdEVs by degradation of CTCs after blood draw is unlikely, as the blood samples were collected in CellSave tubes, which stabilizes the cells in the blood, preventing their degradation ${ }^{46}$.

One way for the clinicians to bring our findings into patient care is by automatically extracting tdEV counts of the already processed samples for CTCs; the tdEV count can serve as a second biomarker to confirm the initial prognosis of the patient based on their CTC counts. However, for the additional stratification of patients with low (favorable) CTC counts reported here, the tdEV cut-off values with the most significant correlation to OS were chosen for each patient cohort. It is recommended that the selected tdEV cutoff values be confirmed in additional studies to derive more unequivocal conclusions.

In case of NSCLC patients, tdEVs are unable to further stratify patients with favorable CTC counts. Moreover, both CTC and tdEV counts, as defined by the CellSearch system, are very low regardless the particular aggressiveness of the specific cancer type. The question why remains open and has been discussed elsewhere ${ }^{47}$. Low expression of EpCAM and/or cytokeratin have been suggested as possible explanations. The Cellsearch yields a CTC recovery of 87-91\% when cells express $1.5-2.3$ * $10^{6}$ EpCAM molecules ${ }^{35}$. However, when EpCAM expression falls to 4.9 - 2.0 *10 $10^{3}$ EpCAM molecules, the respective CTC recovery drops to as low as $2 \%{ }^{35}$. In case of the smaller tdEVs, lower magnetic forces and subsequently EpCAM expression is required for their immunomagnetic isolation. Another critical point is the detection of isolated EpCAM+ CTCs by the expression of CK8, 18 and 19. Colleagues have previously demonstrated that AR+ CTCs isolated by the CellSearch system were not detectable by the CellTracks Analyzer II due to insufficient CK expression ${ }^{48}$. De Wit et al. also reported that in case of NSCLC patients, the inclusion of antibodies targeting more cytokeratins increased the detection of CTCs within the CellSearch cartridges from 41 to $52 \%{ }^{35}$. Therefore, the use of additional antibodies recognizing membrane (EPCAM, VAR2CSA, HsP70, PSMA, HER2, EGFR2) or cytoplasmic (vimentin) markers could lead to increased detection of CTCs and tdEVs that have been already positively selected based on their EpCAM expression.

The inclusion of more specific antibodies in the staining mixture could also aid in the discrimination of tdEVs from EVs secreted from non-tumor epithelial cells in 
the bloodstream of healthy individuals. The reason why only some of the healthy individuals have detectable EVs defined as tdEVs (Figure 3.2) could be explained by a condition of liver inflammation/fibrosis that would result in increased secretion of epithelial EVs. In alignment with that hypothesis, Julich-Haertel et al. demonstrated that individuals with cirrhosis have detectable EpCAM+ AnnexinV+ EVs in similar frequencies to individuals with NSCLC and CRC ${ }^{49}$.

Genotypic characterization of tdEVs in healthy individuals and cancer patients would further confirm their origin. Molecular profiling of the isolated tdEVs could provide us with a mutational snapshot of the tumor during therapies and lead to a better understanding of the underlying pathways and mechanisms that promote progression. Jiang et al. identified AR mutations in CellSearch enriched blood samples of CRPC patients that did not have any CellSearch defined CTCs; some of the AR mutations that were indicating resistance to AR-targeted therapies had been found also in biopsies or autopsies of the respective patients ${ }^{50}$. Furthermore, Marchetti et al. also reported EGFR mutations in CellSearch enriched blood samples of $84 \%$ NSCLC patients, whereas only $41 \%$ of them were positive for Cellsearch defined CTCs ${ }^{51}$. These findings raise the question whether specific gene mutations are present in EpCAM+ CTCs isolated by CellSearch, but missed by the system as CK- or whether these mutations are encapsulated within the EpCAM+, CK+, CD45-, DNA- tdEVs that we report here. Both hypotheses are likely to be true as supported by the presence of CK- CTCs in the CellSearch cartidges ${ }^{48}$ and the presence of most tumor DNA in large $\mathrm{CK}+\mathrm{tdEVs}$ found in the plasma of cancer patients ${ }^{52}$. In addition, it has been reported that relevant gene mutation information is present in the plasma fraction of blood samples, either encapsulated within EVs or as cell-free tumor DNA ${ }^{53,54}$.

Our study has the limitation that all blood samples were centrifuged at $800 \mathrm{~g}$ for $10 \mathrm{~min}$ and the plasma was aspirated before being processed by the CellSearch system; hence, the EpCAM+, CK+, DAPI-, CD45-fraction of tdEVs that we report here was isolated from the blood cell pellet and it has a size range between 1 and $14 \mu \mathrm{m}$. These large tdEVs constitute only a small subset of probably less than $1 \%$ of the total tdEVs that are present in the blood sample before centrifugation, based on the size estimations of secreted EVs from model cancer cell lines ${ }^{55-57}$.

An approach to enrich and further investigate the smaller tdEVs is to run plasma through the CellSearch system. A first attempt indeed showed many CK+tdEVs isolated from the plasma of a CRPC patient by the Cellsearch system, but improvements need to be made for their detection and identification. Further characterization and downstream analysis of the EpCAM enriched tdEVs using gold standard techniques ${ }^{58,67}$, also recommended by the International Society of Extracellular Vesicles $^{59}$, can further elucidate their biophysical properties. Towards that direction, the Cancer-ID consortium was formed to identify and characterize tdEVs and 
elucidate their differences from EVs of different origins. Electron microscopy ${ }^{60,61}$, nanoparticle tracking analysis, flow cytometry ${ }^{62,63}$, Raman spectroscopy ${ }^{64}$, surface plasmon resonance ${ }^{56}$ and atomic force microscopy ${ }^{65}$ have been investigated for the characterization of tdEVs with or without EpCAM pre-enrichment. Principal component analysis of Raman spectra shows clear discrimination between EVs of cancerous cell- and healthy blood cell- origin ${ }^{66}$.

In conclusion, this study shows the simultaneous isolation and detection of CTCs and large (1-14 $\mu \mathrm{m})$ tdEVs in a single assay (CellSearch) maximizing the available data from individual peripheral blood samples of metastatic cancer patients. Importantly, tdEVs have an equivalent prognostic power to CTCs in CRPC, MBC, $\mathrm{mCRC}$ and NSCLC patients and can further stratify patients with low/favorable CTC counts. Furthermore, the non-stringent criteria used to classify an object as a tdEV allows for their reliable and fast automated enumeration using the ACCEPT software without the necessity of time-consuming training and manual scoring by individual users. The presence of tdEVs in higher frequencies, when compared to CTCs, may better reflect the phenotypic heterogeneity of the tumor. That fact together with the increased stability of EVs in circulation render tdEVs to a promising biomarker for clinicians to evaluate the mutational status, transcriptome and proteome of the tumor and the presence of therapeutic targets that could predict treatment responses of patient subsets with or without CTCs. Last but not least, future research of EVs could contribute in the comprehension of the underlying mechanisms of the tumor to develop resistance to treatments and open the path towards the development of new therapies.

\section{Acknowledgements}

The authors acknowledge all patients and healthy donors, the blood samples of whom were used for the accomplishment of the present study. We would also like to thank F.A.W. Coumans for fruitful discussions, C. Breukers for assisting us with the collection of the digitally stored image files and L. Mol and M. Tamminga for providing us with some of the missing $\mathrm{mCRC}$ and NSCLC patient characteristics. 


\section{References}

1. Keup C, Mach P, Aktas B, Tewes M, Kolberg H-C, Hauch S et al. RNA Profiles of Circulating Tumor Cells and Extracellular Vesicles for Therapy Stratification of Metastatic Breast Cancer Patients. Clinical chemistry 2018; 64(7): 1054-1062.

2. Vagner T, Spinelli C, Minciacchi VR, Balaj L, Zandian M, Conley A et al. Large extracellular vesicles carry most of the tumour DNA circulating in prostate cancer patient plasma. Journal of extracellular vesicles 2018; 7(1): 1505403.

3. Taylor DD, Gercel-Taylor C. MicroRNA signatures of tumor-derived exosomes as diagnostic biomarkers of ovarian cancer. Gynecol Oncol 2008; 110(1): 13-21.

4. Mitchell PJ, Welton J, Staffurth J, Court J, Mason MD, Tabi Z et al. Can urinary exosomes act as treatment response markers in prostate cancer? J Transl Med 2009; 7:4.

5. Sequeiros T, Rigau M, Chiva C, Montes M, Garcia-Grau I, Garcia M et al. Targeted proteomics in urinary extracellular vesicles identifies biomarkers for diagnosis and prognosis of prostate cancer. Oncotarget 2017; 8(3): 4960-4976.

6. Nanou A, Coumans FAW, van Dalum G, Zeune LL, Dolling D, Onstenk W et al. Circulating tumor cells, tumor-derived extracellular vesicles and plasma cytokeratins in castrationresistant prostate cancer patients. Oncotarget 2018; 9(27): 19283-19293; doi 10.18632/ oncotarget.25019.

7. Zijlstra A, Di Vizio D. Size matters in nanoscale communication. Nat Cell Biol 2018; 20(3): 228230; doi 10.1038/s41556-018-0049-8.

8. Ciardiello C, Cavallini L, Spinelli C, Yang J, Reis-Sobreiro M, de Candia P et al. Focus on Extracellular Vesicles: New Frontiers of Cell-to-Cell Communication in Cancer. International journal of molecular sciences 2016; 17(2): 175.

9. van Niel G, D'Angelo G, Raposo G. Shedding light on the cell biology of extracellular vesicles. Nat Rev Mol Cell Biol 2018; 19(4): 213-228; doi 10.1038/nrm.2017.125.

10. Coumans FAW, Doggen CJM, Attard G, de Bono JS, Terstappen LWMM. All circulating EpCAM $+C K+C D 45$-objects predict overall survival in castration-resistant prostate cancer. Ann Oncol 2010; 21(9): 1851-1857; doi 10.1093/annonc/mdq030.

11. Larson CJ, Moreno JG, Pienta KJ, Gross S, Repollet M, O'Hara S M et al. Apoptosis of circulating tumor cells in prostate cancer patients. Cytometry A 2004; 62(1): 46-53; doi 10.1002/ cyto.a.20073.

12. Scher HI, Jia XY, de Bono JS, Fleisher M, Pienta KJ, Raghavan D et al. Circulating tumour cells as prognostic markers in progressive, castration-resistant prostate cancer: a reanalysis of IMMC38 trial data. Lancet Oncol 2009; 10(3): 233-239; doi 10.1016/S1470-2045(08)70340-1.

13. Tol J, Koopman M, Miller MC, Tibbe A, Cats A, Creemers GJM et al. Circulating tumour cells early predict progression-free and overall survival in advanced colorectal cancer patients treated with chemotherapy and targeted agents. Ann Oncol 2010; 21(5): 1006-1012; doi 10.1093/ annonc/mdp463. 
14. Cohen SJ, Punt CJA, Iannotti N, Saidman BH, Sabbath KD, Gabrail NY et al. Relationship of circulating tumor cells to tumor response, progression-free survival, and overall survival in patients with metastatic colorectal cancer. Journal of Clinical Oncology 2008; 26(19): 3213-3221; doi 10.1200/Jco.2007.15.8923.

15. Cristofanilli M, Budd GT, Ellis MJ, Stopeck A, Matera J, Miller MC et al. Circulating tumor cells, disease progression, and survival in metastatic breast cancer. N Engl J Med 2004; 351(8): 781-791; doi 10.1056/NEJMoa040766.

16. de Wit S, Zeune LL, Hiltermann TJN, Groen HJM, Dalum Gv, Terstappen LWMM. Classification of Cells in CTC-Enriched Samples by Advanced Image Analysis. Cancers 2018; 10(10).

17. de Wit S, van Dalum G, Lenferink AT, Tibbe AG, Hiltermann TJ, Groen HJ etal. The detection of EpCAM(+) and EpCAM(-) circulating tumor cells. Sci Rep 2015; 5: 12270; doi 10.1038/srep12270.

18. de Wit S, Rossi E, Weber S, Tamminga M, Manicone M, Swennenhuis JF et al. Single tube liquid biopsy for advanced non-small cell lung cancer. Int J Cancer 2018; doi 10.1002/ijc.32056.

19. Allard WJ, Matera J, Miller MC, Repollet M, Connelly MC, Rao C et al. Tumor cells circulate in the peripheral blood of all major carcinomas but not in healthy subjects or patients with nonmalignant diseases. Clinical Cancer Research 2004; 10(20): 6897-6904; doi Doi 10.1158/10780432.Ccr-04-0378.

20. Coumans F, Terstappen L. Detection and Characterization of Circulating Tumor Cells by the CellSearch Approach. Methods Mol Biol 2015; 1347: 263-278; doi 10.1007/978-1-4939-2990-0_18.

21. Budczies J, Klauschen F, Sinn BV, Gyorffy B, Schmitt WD, Darb-Esfahani S et al. Cutoff Finder: a comprehensive and straightforward Web application enabling rapid biomarker cutoff optimization. Plos One 2012; 7(12): e51862; doi 10.1371/journal.pone.0051862.

22. Delves GH, Stewart AB, Cooper AJ, Lwaleed BA. Prostasomes, angiogenesis, and tissue factor. Semin Thromb Hemost 2007; 33(1): 75-79; doi 10.1055/s-2006-958465.

23. Wieckowski E, Whiteside TL. Human tumor-derived vs dendritic cell-derived exosomes have distinct biologic roles and molecular profiles. Immunol Res 2006; 36(1-3): 247-254.

24. Ratajczak J, Wysoczynski M, Hayek F, Janowska-Wieczorek A, Ratajczak MZ. Membranederived microvesicles: important and underappreciated mediators of cell-to-cell communication. Leukemia 2006; 20(9): 1487-1495.

25. Baran J, Baj-Krzyworzeka M, Weglarczyk K, Szatanek R, Zembala M, Barbasz J et al. Circulating tumour-derived microvesicles in plasma of gastric cancer patients. Cancer Immunol Immunother 2010; 59(6): 841-850.

26. Graves LE, Ariztia EV, Navari JR, Matzel HJ, Stack MS, Fishman DA. Proinvasive properties of ovarian cancer ascites-derived membrane vesicles. Cancer Res 2004; 64(19): 7045-7049; doi 10.1158/0008-5472.CAN-04-1800.

27. Sung BH, Ketova T, Hoshino D, Zijlstra A, Weaver AM. Directional cell movement through tissues is controlled by exosome secretion. Nature communications 2015; 6: 7164 .

28. Taylor DD, Gercel-Taylor C. Tumour-derived exosomes and their role in cancer-associated T-cell signalling defects. British journal of cancer 2005; 92(2): 305-311.

29. Peinado H, Aleckovic M, Lavotshkin S, Matei I, Costa-Silva B, Moreno-Bueno G etal. Melanoma exosomes educate bone marrow progenitor cells toward a pro-metastatic phenotype through MET. Nat Med 2012; 18(6): 883-891; doi 10.1038/nm.2753. 
30. Skog J, Wurdinger T, van Rijn S, Meijer DH, Gainche L, Sena-Esteves M et al. Glioblastoma microvesicles transport RNA and proteins that promote tumour growth and provide diagnostic biomarkers. Nat Cell Biol 2008; 10(12): 1470-1476; doi 10.1038/ncb1800.

31. Shedden K, Xie XT, Chandaroy P, Chang YT, Rosania GR. Expulsion of small molecules in vesicles shed by cancer cells: association with gene expression and chemosensitivity profiles. Cancer Res 2003; 63(15): 4331-4337.

32. Caby M-P, Lankar D, Vincendeau-Scherrer C, Raposo G, Bonnerot C. Exosomal-like vesicles are present in human blood plasma. Int Immunol 2005; 17(7): 879-887.

33. George JN, Thoi LL, McManus LM, Reimann TA. Isolation of human platelet membrane microparticles from plasma and serum. Blood 1982; 60(4): 834-840.

34. Zeune L, van Dalum G, Terstappen LWMM, van Gils SA, Brune C. Multiscale Segmentation via Bregman Distances and Nonlinear Spectral Analysis. Siam J Imaging Sci 2017; 10(1): 111-146; doi 10.1137/16m1074503.

35. Zeune LL, de Wit S, Berghuis AMS, MJ IJ, Terstappen L, Brune C. How to Agree on a CTC: Evaluating the Consensus in Circulating Tumor Cell Scoring. Cytometry A 2018; doi 10.1002/ cyto.a.23576.

36. Zeune L, van Dalum G, Decraene C, Proudhon C, Fehm T, Neubauer H et al. Quantifying HER2 expression on circulating tumor cells by ACCEPT. Plos One 2017; 12(10); doi ARTN e0186562 10.1371/journal.pone.0186562.

37. Kang Q Henry NL, Paoletti C, Jiang H, Vats P, Chinnaiyan AM et al. Comparative analysis of circulating tumor DNA stability In K3EDTA, Streck, and CellSave blood collection tubes. Clinical biochemistry 2016; 49(18): 1354-1360.

38. Zeune L. Automated CTC Classification, Enumeration and Pheno Typing: Where Marh meets Biology. Enschede: Medical Cell BioPhysics, University of Twente; 2019.

39. Julich-Haertel H, Urban SK, Krawczyk M, Willms A, Jankowski K, Patkowski W et al. Cancerassociated circulating large extracellular vesicles in cholangiocarcinoma and hepatocellular carcinoma. Journal of hepatology 2017; 67(2): 282-292.

40. Tibbe AG, Miller MC, Terstappen LW. Statistical considerations for enumeration of circulating tumor cells. Cytometry A 2007; 71(3): 154-162; doi 10.1002/cyto.a.20369.

41. Jiang Y, Palma JF, Agus DB, Wang Y, Gross ME. Detection of androgen receptor mutations in circulating tumor cells in castration-resistant prostate cancer. Clin Chem 2010; 56(9): 14921495; doi 10.1373/clinchem.2010.143297.

42. Marchetti A, Del Grammastro M, Felicioni L, Malatesta S, Filice G, Centi I et al. Assessment of EGFR mutations in circulating tumor cell preparations from NSCLC patients by next generation sequencing: toward a real-time liquid biopsy for treatment. Plos One 2014; 9(8): e103883.

43. Crespo M, van Dalum G, Ferraldeschi R, Zafeiriou Z, Sideris S, Lorente D et al. Androgen receptor expression in circulating tumour cells from castration-resistant prostate cancer patients treated with novel endocrine agents. Br J Cancer 2015; 112(7): 1166-1174; doi 10.1038/ bjc.2015.63.

44. Vagner T, Spinelli C, Minciacchi VR, Balaj L, Zandian M, Conley A et al. Large extracellular vesicles carry most of the tumour DNA circulating in prostate cancer patient plasma. $J$ Extracell Vesicles 2018; 7(1): 1505403; doi 10.1080/20013078.2018.1505403. 
45. Taback B, Hoon DSB. Circulating nucleic acids and proteomics of plasma/serum: clinical utility. Ann N Y Acad Sci 2004; 1022: 1-8.

46. Pasquale R, Fenizia F, Esposito Abate R, Sacco A, Esposito C, Forgione L et al. Assessment of high-sensitive methods for the detection of EGFR mutations in circulating free tumor DNA from NSCLC patients. Pharmacogenomics 2015; 16(10): 1135-1148.

47. Manicone M, Poggiana C, Facchinetti A, Zamarchi R. Critical issues in the clinical application of liquid biopsy in non-small cell lung cancer. J Thorac Dis 2017; 9(Suppl 13): S1346-S1358; doi 10.21037/jtd.2017.07.28.

48. Lee W, Nanou A, Rikkert L, Coumans FAW, Otto C, Terstappen LWMM et al. Label-Free Prostate Cancer Detection by Characterization of Extracellular Vesicles Using Raman Spectroscopy. Anal Chem 2018; 90(19): 11290-11296; doi 10.1021/acs.analchem.8bo1831.

49. Gool EL, Stojanovic I, Schasfoort RBM, Sturk A, van Leeuwen TG, Nieuwland R et al. Surface Plasmon Resonance is an Analytically Sensitive Method for Antigen Profiling of Extracellular Vesicles. Clin Chem 2017; 63(10): 1633-1641; doi 10.1373/clinchem.2016.271049.

50. Coumans F, van Dalum G, Terstappen LWMM. CTC Technologies and Tools. Cytometry Part A : the journal of the International Society for Analytical Cytology 2018; 93(12): 1197-1201.

51. Beekman P, Enciso-Martinez A, Rho HS, Pujari SP, Lenferink A, Zuilhof $\mathrm{H}$ et al. Immunocapture of extracellular vesicles for individual multi-modal characterization using AFM, SEM and Raman spectroscopy. Lab Chip 2019; 19(15): 2526-2536; doi 10.1039/c9lcooo81j.

52. Lee W, Nanou A, Rikkert L, Coumans FAW, Otto C, Terstappen L et al. Label-Free Prostate Cancer Detection by Characterization of Extracellular Vesicles Using Raman Spectroscopy. Anal Chem 2018; 90(19): 11290-11296; doi 10.1021/acs.analchem.8bo1831.

53. Wu YT, Deng WT, Klinke DJ. Exosomes: improved methods to characterize their morphology, RNA content, and surface protein biomarkers. Analyst 2015; 140(19): 6631-6642; doi 10.1039/ C5anoo688k. 


\section{Supplementary Data}

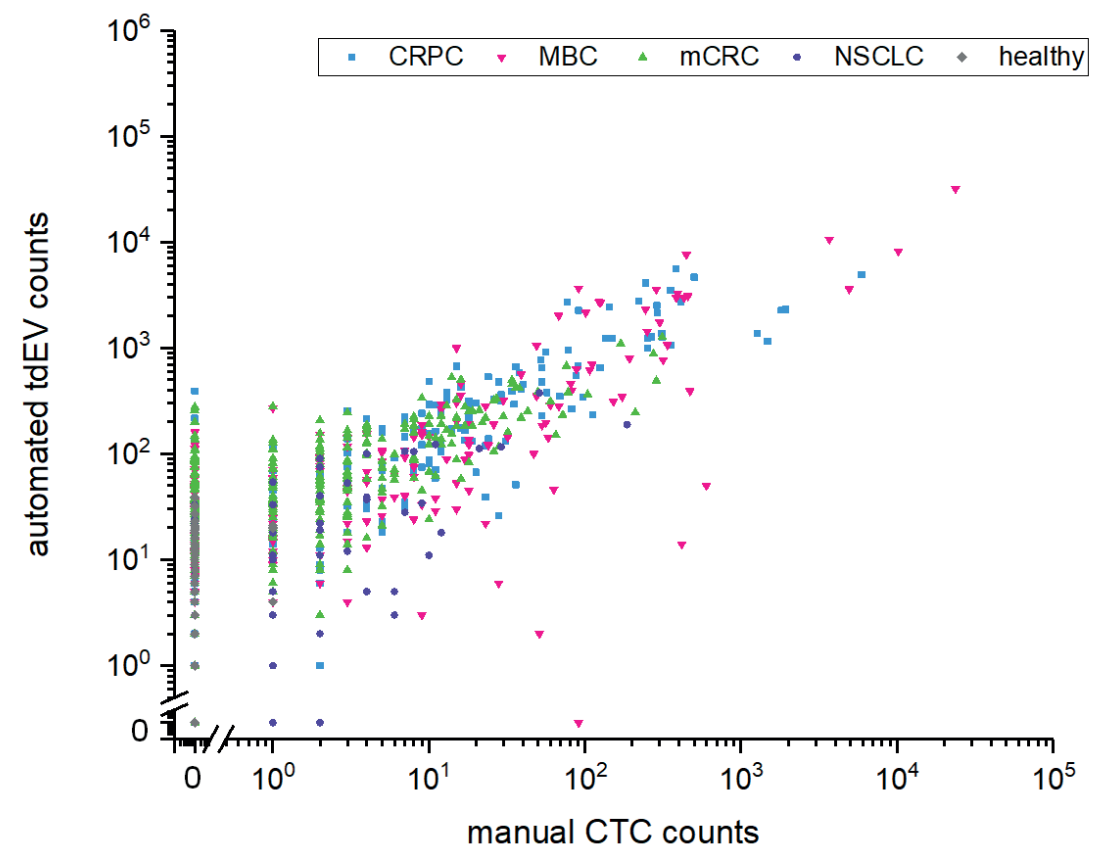

Supplementary Figure S3.1. Scatter plot showing the correlation between manual CTC and automated tdEV counts in four cancer types and healthy donors. Each group is indicated in a different color as shown in the legend. 
A. CTCs for full data set
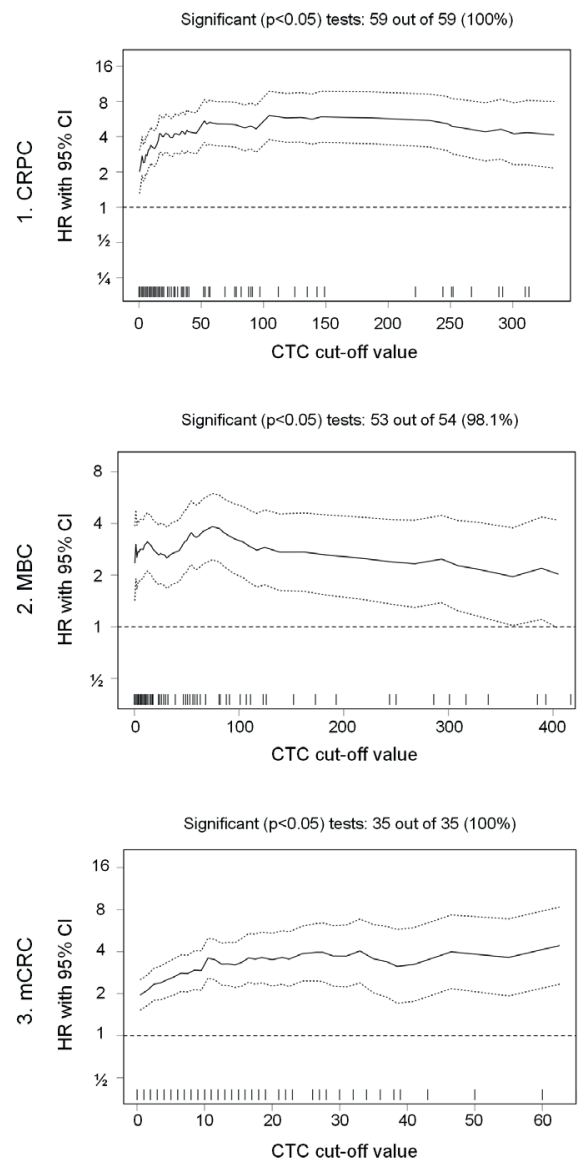

Significant $(p<0.05)$ tests: 6 out of $6(100 \%)$

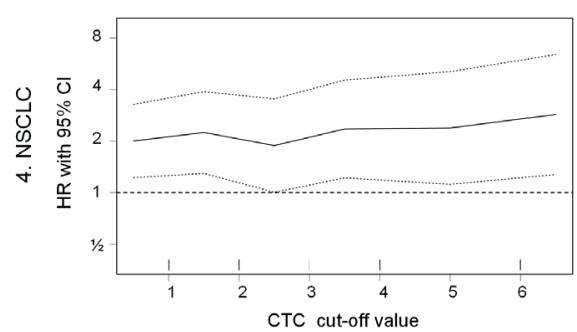

B. tdEVs for full dataset

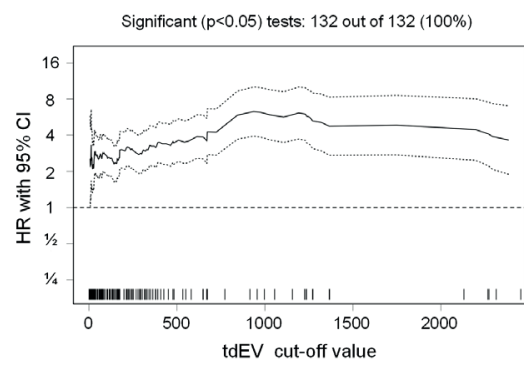

Significant $(p<0.05)$ tests: 113 out of $120(94.2 \%)$

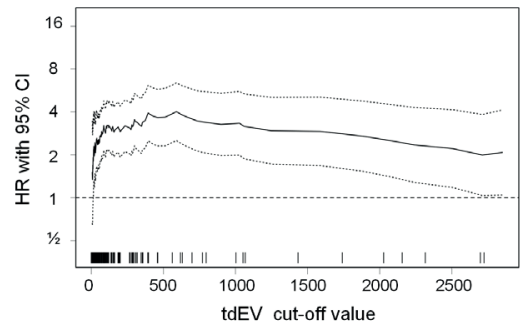

Significant $(p<0.05)$ tests: 147 out of $149(98.7 \%)$

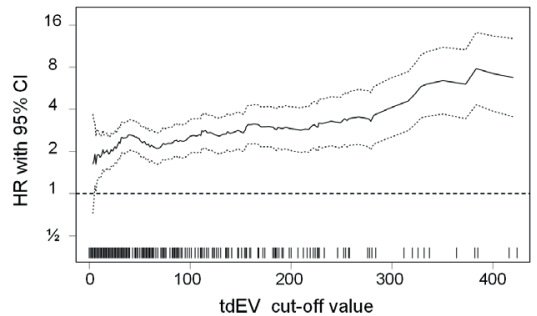

Significant $(p<0.05)$ tests: 20 out of $30(66.7 \%)$

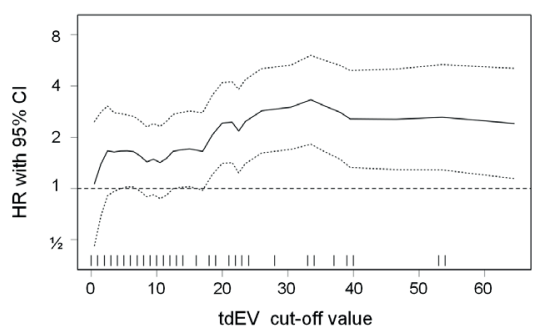

Supplementary Figure S3.2. Overview plots of Hazard Ratios (HRs), including 95\% CIs, for overall survival (OS) for all possible cut-off values for CTC counts (A) and tdEV counts (B) in each cancer type. These plots were generated using the web application Cutoff Finder. CTCs and tdEVs were significant for a wide range of cut-off values in CRPC (Panels IA and 1B), MBC (Panels 2A and 2B), mCRC (Panels $3 \mathrm{~A}$ and $3 \mathrm{~B}$ ), and NSCLC (Panels $4 \mathrm{~A}$ and $4 \mathrm{~B}$ ). 
Receiver Operating Characteristic Curves (ROC)

A. Classification variable: survival < median oS
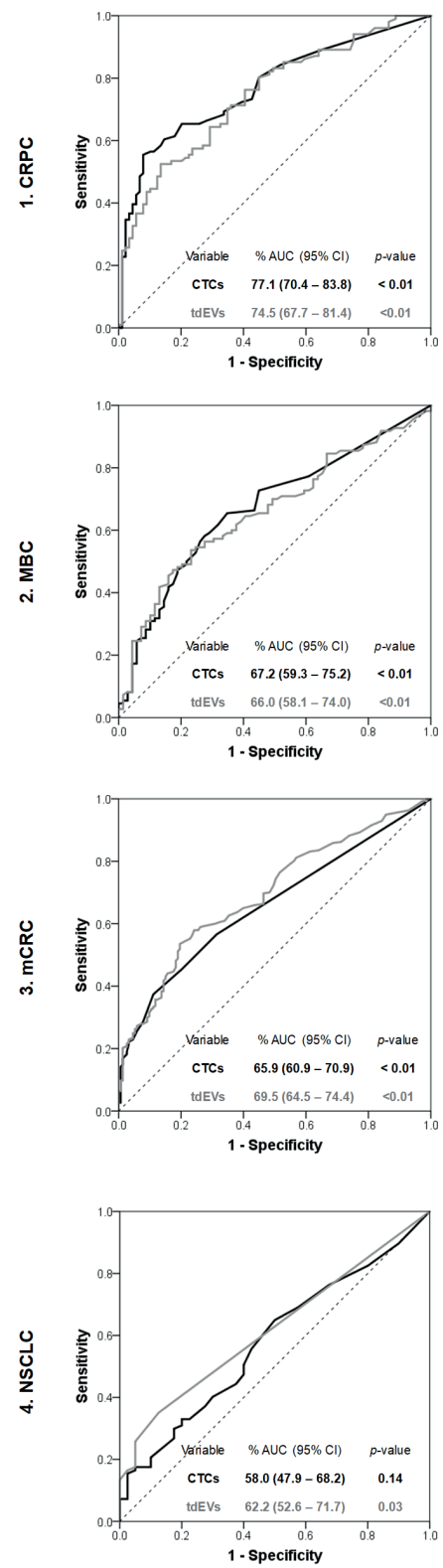

B. Classification variable: death
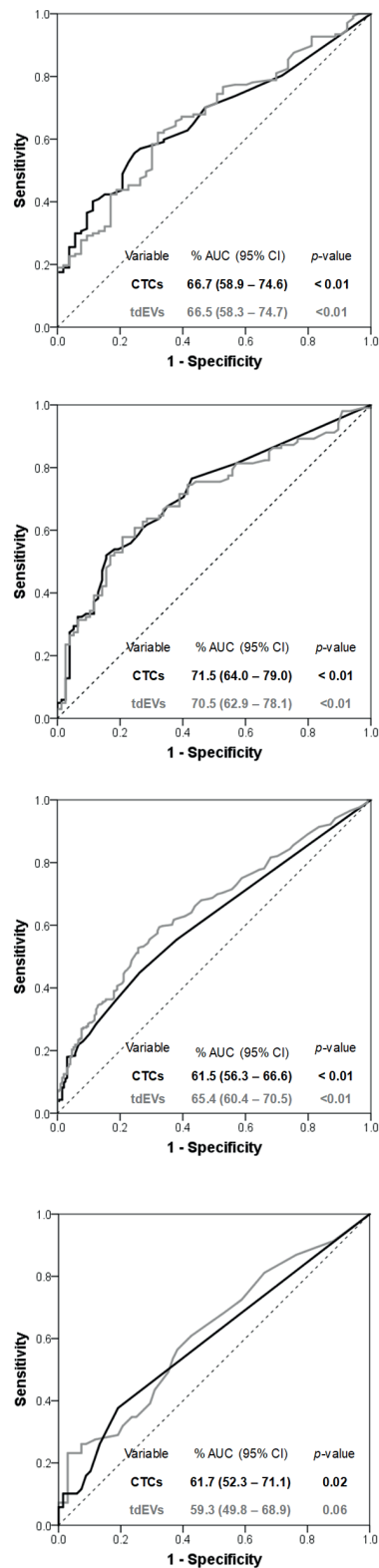

Supplementary Figure S3.3. Receiver Operating Characteristic (ROC) curves of CTCs (in black) and tdEVs (in grey) treating as a classification variable A. survival time dichotomized by the median OS time of the respective patient cohort and B. death. 
Supplementary Table S3.1. 137 NSCLC patient samples included in the present study

\begin{tabular}{llll}
\hline N patients & Additional labeling & Additional staining & Previously reported \\
\hline 45 & Marker 1 & Wheat-germ agglutinin- Alexa 488 & (de Wit et al., 2018b) \\
& Marker 2 & CD16-PerCP & \\
12 & Marker 2 & CD16-PerCP & not \\
24 & Marker 1 & CK1-8, 10, 14, 15, 16, 19, 20-FITC & (de Wit et al., 2015) \\
& Marker 2 & CD16-PerCP & \\
56 & Marker 2 & CD16-PerCP & (de Wit et al., 2018a) \\
\hline
\end{tabular}


Supplementary Table S3.2. Univariable cox proportional hazards regression analysis

\begin{tabular}{|c|c|c|c|}
\hline & Variables in equation & HR $(95 \% \mathrm{CI})$ & p-value \\
\hline \multirow{10}{*}{$\begin{array}{l}\text { U: } \\
\text { 접 }\end{array}$} & age & $1.02(1.00-1.04)$ & 0.025 \\
\hline & ECOG & $2.33(1.75-3.10)$ & $<0.001$ \\
\hline & CTCs, per log unit & $1.72(1.48-2.00)$ & $<0.001$ \\
\hline & tdEVs, per log unit & $2.42(1.90-3.07)$ & $<0.001$ \\
\hline & haemoglobin, per $\mathrm{g} / \mathrm{dL}$ & $0.69(0.62-0.77)$ & $<0.001$ \\
\hline & PSA, per log unit & $1.27(1.00-1.62)$ & 0.050 \\
\hline & ALP, per log unit & $3.70(2.31-5.93)$ & $<0.001$ \\
\hline & albumin, per log unit & $0.01(0.00-0.02)$ & 0.002 \\
\hline & testosterone, per log unit & $0.71(0.35-1.42)$ & 0.328 \\
\hline & LDH, per log unit & $26.54(11.28-62.48)$ & $<0.001$ \\
\hline \multirow{11}{*}{ U } & age & $1.00(0.98-1.01)$ & 0.526 \\
\hline & ECOG & $1.81(1.39-2.36)$ & $<0.001$ \\
\hline & CTCs, per log unit & $1.62(1.38-1.90)$ & $<0.001$ \\
\hline & tdEVs, per log unit & $1.92(1.53-2.40)$ & $<0.001$ \\
\hline & ER status & $1.48(0.97-2.24)$ & 0.068 \\
\hline & PR status & $1.57(1.06-2.34)$ & 0.025 \\
\hline & HER2/neu status & $1.43(0.89-2.30)$ & 0.136 \\
\hline & number of metastatic sites & $1.24(1.07-1.43)$ & 0.003 \\
\hline & time to metastasis & $0.97(0.93-1.00)$ & 0.067 \\
\hline & line of therapy & $1.06(1.00-1.12)$ & 0.052 \\
\hline & type of therapy & $0.81(0.67-0.97)$ & 0.021 \\
\hline \multirow{9}{*}{ 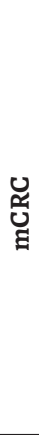 } & age & $1.01(1.00-1.03)$ & 0.039 \\
\hline & ECOG & $1.51(1.18-1.92)$ & 0.001 \\
\hline & gender & $0.99(0.77-1.27)$ & 0.936 \\
\hline & treatment arm & $1.14(0.90-1.46)$ & 0.284 \\
\hline & prior adjuvant chemotherapy & $1.34(0.93-1.93)$ & 0.117 \\
\hline & $>1$ affected organs & $0.76(0.59-0.98)$ & 0.035 \\
\hline & abnormal LDH & $0.59(0.46-0.75)$ & $<0.001$ \\
\hline & CTCs, per log unit & $1.65(1.44-1.90)$ & $<0.001$ \\
\hline & tdEVs, per log unit & $2.53(1.99-3.20)$ & $<0.001$ \\
\hline \multirow{6}{*}{$\begin{array}{l}\text { U్త } \\
\text { జ } \\
\text { Z }\end{array}$} & age & $1.03(1.00-1.06)$ & 0.024 \\
\hline & ECOG & $2.04(1.36-3.04)$ & 0.001 \\
\hline & gender & $0.59(0.36-0.95)$ & 0.030 \\
\hline & type of therapy & $0.98(0.74-1.31)$ & 0.907 \\
\hline & CTCs, per log unit & $1.66(1.24-2.23)$ & 0.001 \\
\hline & tdEVs, per log unit & $1.52(1.06-2.19)$ & 0.024 \\
\hline
\end{tabular}


Supplementary Table S3.3. Multivariable cox proportional hazards regression analysis

\begin{tabular}{|c|c|c|c|}
\hline & Variables in equation & HR $(95 \% \mathrm{CI})$ & p-value \\
\hline \multirow{5}{*}{ 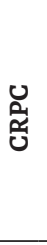 } & LDH, per log unit & $7.01(2.47-19.96)$ & $<0.001$ \\
\hline & tdEVs, per log unit & $1.87(1.42-2.47)$ & $<0.001$ \\
\hline & ECOG status & $1.62(1.19-2.20)$ & 0.002 \\
\hline & haemoglobin, per g/dL & $0.80(0.70-0.90)$ & $<0.001$ \\
\hline & age & $1.03(1.01-1.05)$ & 0.003 \\
\hline \multirow{3}{*}{ ט } & ECOG status & $1.76(1.34-2.30)$ & $<0.001$ \\
\hline & CTCs, per log unit & $1.62(1.37-1.92)$ & $<0.001$ \\
\hline & number of metastatic sites & $1.22(1.05-1.41)$ & 0.008 \\
\hline \multirow{4}{*}{ U્口 } & tdEVs, per log unit & $1.92(1.37-2.68)$ & $<0.001$ \\
\hline & ECOG status & $1.36(1.06-1.74)$ & 0.016 \\
\hline & CTCs, per log unit & $1.23(1.01-1.49)$ & 0.041 \\
\hline & age & $1.02(1.01-1.04)$ & 0.005 \\
\hline \multirow{3}{*}{$\begin{array}{l}\text { U্త } \\
\text { ֻ } \\
\text { Z }\end{array}$} & ECOG status & $1.90(1.26-2.86)$ & 0.002 \\
\hline & CTCs, per log unit & $1.47(1.05-2.07)$ & 0.026 \\
\hline & age & $1.04(1.01-1.06)$ & 0.010 \\
\hline
\end{tabular}




\title{
Chapter 4
}

\section{Leukocyte-derived Extracellular Vesicles in blood with and without EpCAM enrichment}

\author{
Afroditi Nanou, Leonie L. Zeune, Leon W.M.M.M Terstappen
}

Cells 2019, 8 (8), 937.

\begin{abstract}
Large tumor-derived Extracellular Vesicles (tdEVs) in EpCAM enriched blood fluorescently labelled with DAPI, Cytokeratin-PE and CD45-APC of metastatic prostate, breast, colorectal and non-small cell lung cancer patients are negatively associated with the overall survival of the patients. Here, we investigated whether, similarly to tdEVs, also leukocyte-derived EVs (ldEVs) can be detected in EPCAM enriched blood. Presence of ldEVs and leukocytes in image data sets of EpCAM enriched samples of 25 healthy individuals and 75 metastatic cancer patients was evaluated using the ACCEPT software. Large ldEVs could indeed be detected, but in contrast to the 20 -fold higher frequency of tdEVs as compared to Circulating Tumor Cells (CTCs), ldEVs were present in a 5 -fold lower frequency as compared to leukocytes. To evaluate whether these ldEVs pre-exist in the blood or are formed during the CellSearch procedure, blood of healthy individuals without EpCAM enrichment was labelled with the nuclear dye Hoechst and fluorescently tagged monoclonal antibodies recognizing the leukocyte-specific CD 45 , platelet-specific CD61 and red blood cell- specific CD235a. Fluorescence microscopy imaging using a similar setup as the CellSearch was performed and demonstrated the presence of a similar population of ldEVs present at a 3 -fold lower frequency as compared to leukocytes.
\end{abstract}




\subsection{Introduction}

During the last decades, Extracellular Vesicles (EVs) have emerged as promising disease biomarkers bearing similar membrane and cargo composition as their originating cells ${ }^{1-3}$. Importantly, for nucleic acid analysis, the membrane encapsulated nucleic acid cargo is protected from enzymatic degradation and consequently, it can circulate for longer time compared to cell free DNA (cfDNA) ${ }^{4,5}$. In case of cancer, the presence of nucleic acids (DNA, mRNA and miRNA) within tumor-derived EVs (tdEVs) and proteins within or on tdEV membranes can provide information of the predisposition of the tumor to metastasize in specific organs and guide treatment monitoring of the patients to block metastasis and cancer progression ${ }^{5-9}$. It has been demonstrated that EVs in biofluids of cancer patients are significantly elevated when compared to the respective numbers of healthy individuals ${ }^{10,11}$. However, to our knowledge, there is no data available in regards to the composition of the redundant EVs in blood of cancer patients. The recent in vivo studies of Ricklefs et al. using GFP-labeled brain tumors in mice showed that less than $0.5 \%$ of the total circulating EVs were GFP+ ${ }^{10}$. That finding implies that more cell types secrete EVs in response to the present tumor contributing to the final EV pool detected in biofluids of cancer patients. Furthermore, the preanalytical steps of sample processing determine the EV populations to be analyzed and could lead to biased conclusions. The majority of research groups is only interested in exosomes that constitute the smallest subclass of EVs as they consider them products of active cell secretion; therefore, they are using differential centrifugation steps to get rid of other EV subclasses, collect the exosome fraction as a pellet from the final ultracentrifugation step and label them with antibodies recognizing generic exosome-enriched biomarkers, mainly tetraspanins, such as CD81, CD9 and CD63 to identify them ${ }^{10,12}$. Nevertheless, EV subclasses of larger size (microvesicles, oncosomes and apoptotic bodies) ${ }^{13}$ have been reported to be bioactive with a wide spectrum of functions depending on their cells of origin ${ }^{14}$. Importantly, Vagner et al. reported the presence of DNA in large tdEVs reflecting the genetic aberrations of the tumor; a finding that highlights their promising potential in the liquid biopsy field ${ }^{15}$. Padda et al. also demonstrated that the majority of PSMA+ EVs in plasma of prostate cancer patients derive directly from the plasma membrane and have a larger size ${ }^{16}$; hence, these clinically important populations are missed by solely the exosome analysis. Very few studies have investigated the isolation and downstream characterization of specifically tdEVs from patient samples using immuno-affinity techniques ${ }^{17,18}$. Recently, we showed that large tdEVs, immunomagnetically isolated based on their EpCAM expression together with Circulating Tumor Cells (CTCs) by the CellSearch system from blood of metastatic prostate, breast, colorectal and nonsmall cell lung cancer patients have equivalent prognostic power to CTCs ${ }^{18,19}$. These observations were enabled through the availability of the open-source ACCEPT image analysis program, which allows for the exploration and enumeration in a single level 
of all different classes of objects detected in the fluorescence images in an automated, fast and reproducible manner, free of the subjectivity and bias of different operators ${ }^{20,21}$. However, it is not clear whereas our previously reported large tdEVs are a result of fragmentation of CTCs during the immunomagnetic EpCAM enrichment and washing steps that the CellSearch system is using or whether they pre-exist in the blood samples of cancer patients. Their rare frequency in combination with the abundance of blood cells and EVs of different origins prevent us from addressing that question by labeling of blood samples without any pre-enrichment steps and subsequent enumeration from fluorescence images. In this study, we identified in the digitally stored CellSearch images some CD45+, DAPI-, CK- objects of similar size to tdEVs that we baptized leukocyte-derived Extracellular Vesicles (ldEVs). We addressed the question of whether large ldEVs pre-exist in the blood of individuals without EpCAM enrichment or they are by-products of cell fragmentation by the CellSearch procedure. Towards that direction, we labeled blood samples of healthy individuals with the nuclear dye Hoechst and fluorophore-conjugated antibodies against the leukocytespecific CD45, the platelet-specific CD61 and the red blood cell- specific CD235a without any pre-enrichment or pre-analytical steps. The samples were imaged using a fluorescence microscope with a 10x/0.45 NA objective to enable fair comparison of the image datasets acquired by the CellTracks Analyzer II of the CellSearch system ${ }^{22}$.

\subsection{Materials and Methods}

\subsubsection{Immunofluorescence image data sets of EpCAM enriched cells and extracellular vesicles of 25 healthy individuals and 75 metastatic cancer patients}

100 digitally stored CellSearch image data sets corresponding to EpCAM enriched blood samples of 25 healthy individuals, 25 metastatic prostate (CRPC), 25 colorectal (mCRC) and 25 non-small cell lung (NSCLC) cancer patients before the initiation of a new therapy, were used for this analysis. The EpCAM enriched leukocytes and large leukocyte-derived EVs present in these fluorescence images were enumerated. The CRPC and MCRC patients had participated in the retrospective IMMC38 and CAIRO II clinical studies respectively.

Briefly, the EpCAM+CTCs and tdEVs were positively selected by ferrofluids conjugated to an antibody regognizing the extracellular epitope of EpCAM (clone VU1D9) from $7.5 \mathrm{~mL}$ of blood of cancer patients using the CellSearch system (Menarini Silicon Biosystems, Huntingdon Valley PA, USA), as previously described ${ }^{23}$. Following EpCAM immunomagnetic enrichment, the suspension was incubated with the nuclear dye DAPI and antibodies against the epithelial-specific cytokeratins 8, 18 and 19 (clone C11) conjugated to phycoerythrin (PE) and an antibody against the leukocyte-specific cluster of differentiation CD45 conjugated to allophycocyanin (APC). The suspension 
was transferred to a cartridge placed within a magnest that allowed for a homogeneous distribution on a focal plane of the ferrofluids and the EpCAM enriched objects ${ }^{24}$. The cartridges were imaged using a semi-automated 10x/0.45 NA objective fluorescence microscope, the CellTracks Analyzer II, as previously described ${ }^{23}$.

\subsubsection{Blood samples of 10 healthy individuals}

EDTA blood samples from 10 anonymous healthy individuals were obtained after written informed consent from the TNW-ECTM-donor service (University of Twente, Enschede, The Netherlands). The frequencies of white blood cells, red blood cells and platelets were assessed using a hematology analyzer (Beckman Coulter, California, USA). The samples were processed at the same day of the drawing.

\subsubsection{Immunofluorescence imaging of cells and extracellular vesicles in whole blood samples}

10-20 $\mu \mathrm{l}$ of EDTA blood samples of 10 different healthy individuals were 10x diluted in $0.2 \mu \mathrm{m}$ filtered $1 \% \mathrm{w} / \mathrm{v}$ bovine serum albumin (BSA) in phosphate buffered saline (PBS) solution. Blood was incubated with the nuclear dye Hoechst 33342 (Invitrogen, cat. \# H3570), the fluorescently tagged monoclonal antibodies CD45-PerCP (clone HI30 Invitrogen, cat. \# MHCD4531) recognizing leukocytes, CD235a-Alexa Fluor ${ }^{\circledR} 647$ (clone YTH89.1, bio-rad, MCA506A647) antibodies recognizing erythrocytes and CD61-Alexa Fluor ${ }^{\circledR} 488$ (clone Y2.51, bio-rad, cat. \# MCA 2588A488) antibodies recognizing platelets. The final concentrations used were $4.0 \mu \mathrm{g} / \mathrm{ml}$ Hoechst, $0.5 \mu \mathrm{g} / \mathrm{ml} \mathrm{CD} 45-P e r C P, 2.5 \mu \mathrm{g} /$ $\mathrm{ml} \mathrm{CD} 235 \mathrm{a}$-Alexa 647 and $0.6 \mu \mathrm{g} / \mathrm{ml}$ CD61-Alexa 488. The samples were incubated with the antibodies at $37^{\circ} \mathrm{C}$ for $1-2 \mathrm{~h}$ and stored at $4^{\circ} \mathrm{C}$ until further processing. Subsequently, the samples were further diluted to a final dilution of 500x. $10 \mu \mathrm{l}$ of the diluted sample (corresponding to $0.02 \mu \mathrm{l}$ of undiluted blood) were loaded in a well of EasyCount ${ }^{\mathrm{TM}}$ Slide- $6^{\text {TM }}$ (Immunicon Corp., Huntingdon Valley, PA). 4-6 technical replicates of samples were used to assess reproducibility of the measurements. Image data sets of 55-65 frames/channel were acquired to cover the whole surface of each well using a semi-automated inverted fluorescence microscope (Eclipse Ti-E, Nikon Instruments, Amsterdam, The Netherlands) equipped with a 10x/0.45 NA objective, a camera (Orca flash 4.0 LT, C11440, Hamamatsu, Almere, The Netherlands) and fluorescence filter cubes (DAPI, FITC, PerCP, APC filter sets for the detection of Hoechst, CD61-Alexa 488, CD45-PerCP and CD235a-Alexa 647, respectively). The operator determined 3 corners of the surface to be scanned and adjusted the focus in 4 points distributed throughout each well. The exposure times used for the imaging were $20 \mathrm{msec}$ for DAPI, $400 \mathrm{msec}$ for PerCP, $500 \mathrm{msec}$ for FITC, $1000 \mathrm{msec}$ for APC and $500 \mathrm{msec}$ for brightfield. Few images were obtained using a 60x/0.70 NA objective and the same exposure times for comparison. However, only the images obtained with the 10x objective were used as an input for the enumeration of the different populations to allow a fair comparison with the images of the CellTracks Analyzer II. An example of a frame acquired with the 10x and 60x objectives is shown in Supplementary Figure S4.1. 


\subsubsection{Automated enumeration of objects in immunofluorescence images using the open-source ACCEPT software}

All immunofluorescence image data sets, obtained with the 10x/0.45 NA objective microscopes, were processed with the open-source ACCEPT software (http://github. com/LeonieZ/ACCEPT). The software detects all present objects, larger than 4 pixels, and extracts for each of them 10 morphological and fluorescence signal intensity measurements per fluorescence channel ${ }^{20}$. The user can design linear gates based on these features to define the classes of their interest and enumerate the objects falling within them ${ }^{18,25}$. The application of the same gates for all different samples allows elimination of inter- and intra- operator variations leading subsequently, to a more objective consensus ${ }^{26}$.

For the CellSearch generated images, gates for the enumeration of leukocyte-derived Extracellular Vesicles (ldEVs) and leukocytes were applied. The gates are summarized in Table 4.1. For the image data sets corresponding to the EasyCount Slides-6, gates for the enumeration of red blood cells, leukocytes, platelets and ldEVs were used and are summarized in Table 4.1.

\subsection{Results}

\subsubsection{Detection of ldEVs in EpCAM enriched blood samples of healthy individuals and metastatic cancer patients}

After careful examination of the immunofluorescence images of the CellSearch cartridges, CD45+, DAPI-, CK- objects, that resemble in size our previously reported CD45-, DAPI-, CK+ tdEVs ${ }^{18,27}$, can be observed. We baptized these objects leukocytederived Extracellular Vesicles (ldEVs). Examples of single ldEV events in EpCAM enriched samples by the CellSearch system are shown in Figure 4.1 next to some examples of leukocytes as a reference to their size and $C D 45$ phenotype. The observation of the presence of these ldEVs in the CellSearch cartridges raised questions about their formation: are they fragments of leukocytes formed during the CellSearch procedure or do they pre-exist in the blood circulation? 


\section{Leukocytes and IdEVs in EpCAM enriched blood}

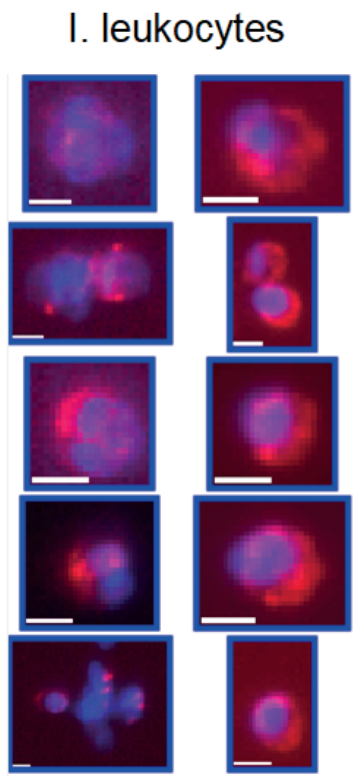

II. IdEVs
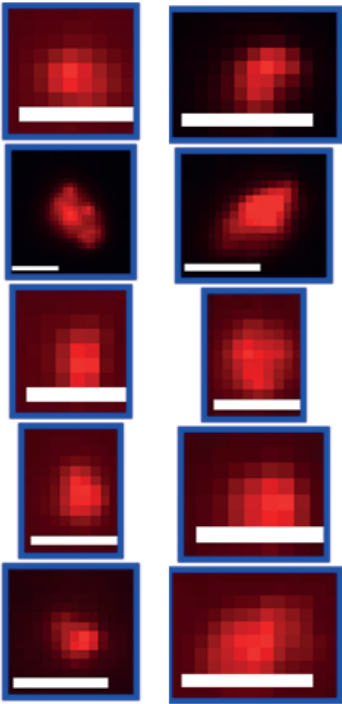

Figure 4.1. Thumbnails of I. leukocytes and II. ldEVs detected in EpCAM enriched blood samples. Red color represents CD45 and blue DAPI. Scale bars indicate $6.4 \mu \mathrm{m}$.

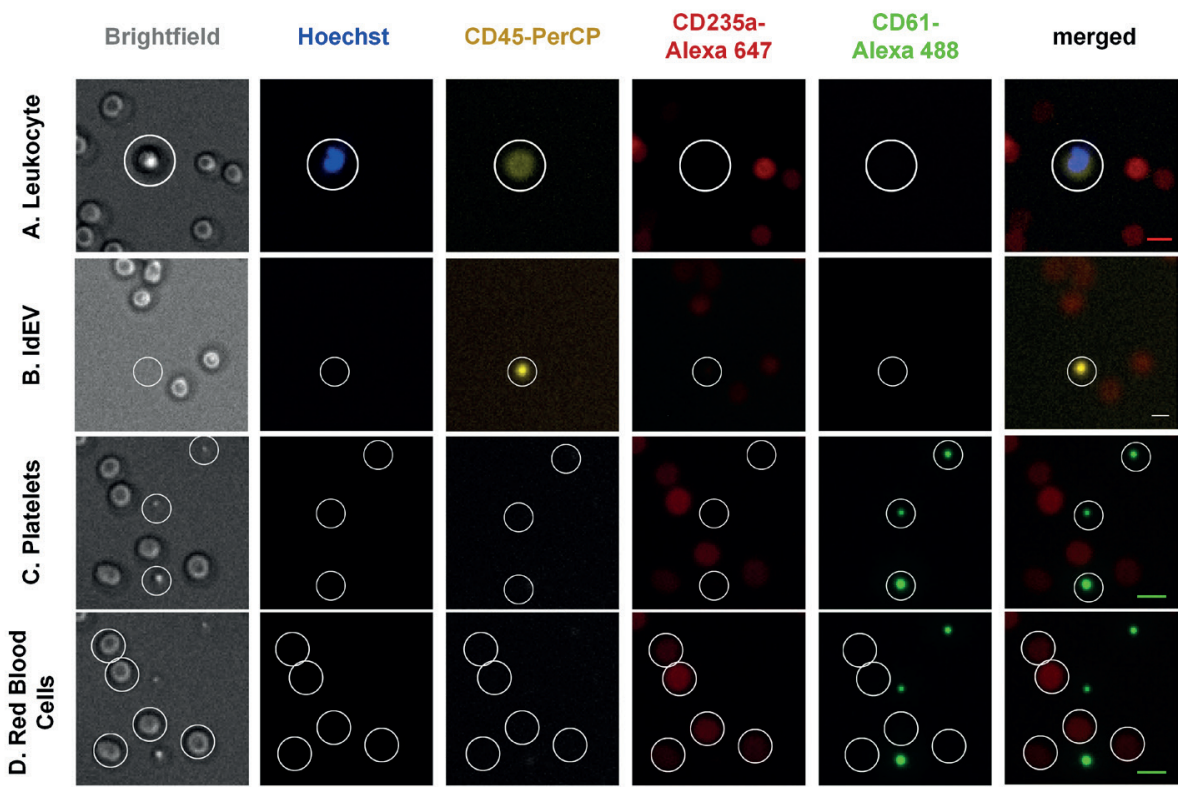

Figure 4.2. Bright field and immunofluorescence images of leukocytes, ldEVs, platelets and red blood cells in blood samples without EpCAM enrichment. Scale bars in the merged IF images indicate $10 \mu \mathrm{m}$. 


\subsubsection{Detection of cell and extracellular vesicle classes in blood of healthy individuals without EpCAM enrichment}

In order to address the aforementioned question, blood samples of healthy individuals were labeled with Hoechst, CD45-PerCP, CD61-Alexa 488 and CD235a-Alexa 647 and were imaged with a similar fluorescence microscope as the CellTracks Analyzer II. No pre-enrichment or washing steps were used in order to minimize cell fragmentation or/ and activation. The inclusion of the aforementioned antibodies allowed the detection of four different classes of objects in whole blood of healthy individuals, namely leukocytes, platelets, red blood cells and ldEVs, as shown in Figure 4.2. Leukocytes are defined as nucleated CD45+, CD61-, CD235a- cells of a size between 7 and $20 \mu \mathrm{m}$ (Panel A); leukocyte-derived Extracellular Vesicles (ldEVs) as CD45+, CD61-, CD235objects without a nucleus and of undefined size as shown in the respective brightfield image (Panel B); platelets as CD45-, CD61+, CD235a- objects without a nucleus of size between 2 and $5 \mu \mathrm{m}$ (Panel $\mathrm{C}$ ) and red blood cells as CD45-, CD61-, CD235a+ cells without a nucleus and a size range between 6 and $10 \mu \mathrm{m}$ (Panel D). In Panel C, three platelets are shown, of which the middle one is clearly smaller and with a lower expression of CD61; examination at higher magnification would allow for a better identification of the smaller size platelets but no discrimination could be made between small platelets and larger platelet-derived EVs. The presence of ldEVs in blood samples without preenrichment (Panel B) confirmed their pre-existence in whole blood

\subsubsection{ACCEPT gates for the automated enumeration of different classes in blood with and without EpCAM enrichment.}

In order to acquire the absolute counts of the different classes from each data set of the healthy individuals in a fast and unbiased manner, we processed all data sets with the open-source ACCEPT software. Based on the aforementioned characteristics of the different classes, we developed linear gates to automatically enumerate the objects falling within each class. The gates are summarized in Table 4.1. Three examples of objects per class are shown in Figure 4.3 (Panel A). The objects that fall into each class are depicted in blue dots in the scatter plots (Panel B) showing the mean Hoechst intensity versus the mean CD45-PerCP intensity and the mean CD61-Alexa 488 intensity versus the mean CD235a-Alexa 647 intensity. Objects falling in the "leukocyte" gate are double positive for CD45 and Hoechst and negative for CD61 and CD235a, (Panel B1); ldEVs are positive only for CD45 (Panel B2); platelets are only positive for CD61 (Panel B3) and red blood cells are only positive for CD235a (Panel B4).

In order to achieve a fair comparison between the leukocyte and IdEV counts detected in blood with and without EPCAM enrichment, very similar ACCEPT gates were developed for the automated enumeration of the two classes applied in the different image data sets. The gates can be found in Table 4.1. 


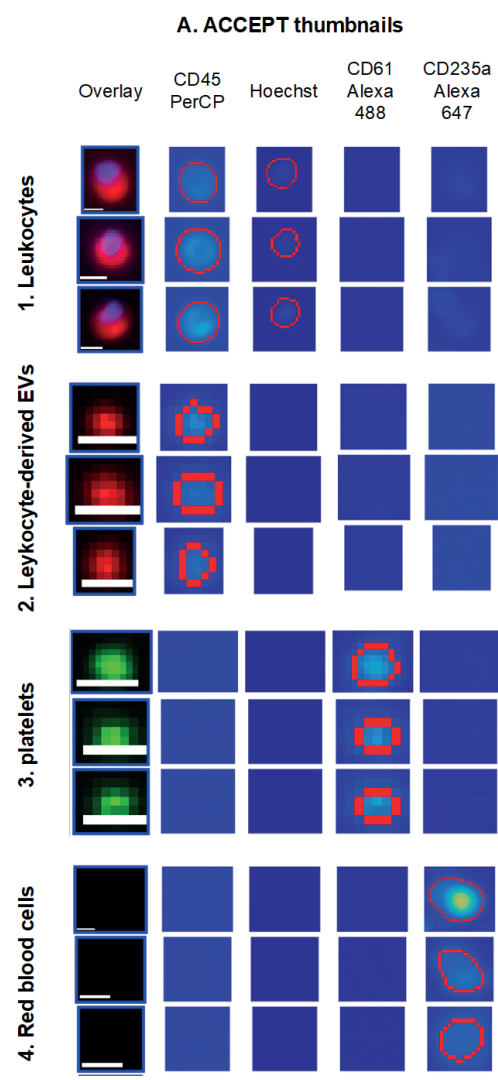

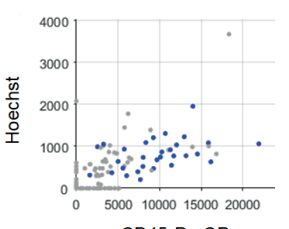

CD45-PerCP
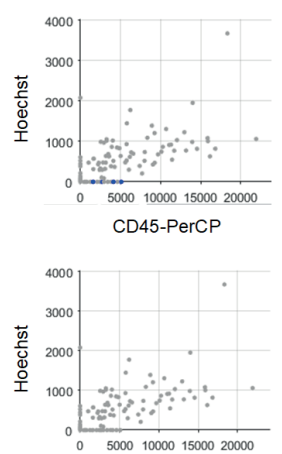

CD45-PerCP

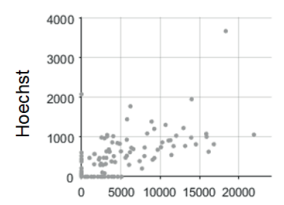

CD45-PerCP
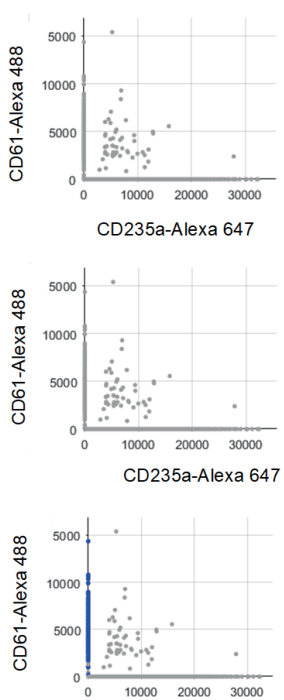

CD235a-Alexa 647

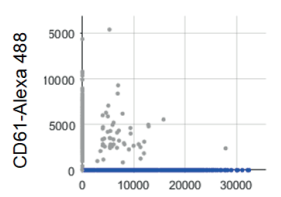

CD235a-Alexa 647

Figure 4.3. Examples of objects detected in the immunofluorescence image data sets of blood samples without EpCAM enrichment. The objects fall in the ACCEPT gates, the names of which are indicated vertically. Panel A shows examples of ACCEPT thumbnails. Scale bars indicate $6.4 \mu \mathrm{m}$. Panel B shows scatter plots of Mean Intensity of Hoechst versus Mean Intensity of CD45-PerCP and Mean Intensity of CD61-Alexa 488 versus Mean Intensity of CD235a-Alexa 647. Blue dots represent single events falling in the respective gate.

The size threshold of $20 \times 20 \mu \mathrm{m}^{2}$ in case of the "leukocyte" gate that was applied in blood samples with no enrichment was removed in the respective gate of EpCAM enriched samples because within the CellSearch cartridges, there are many leukocytes present in close proximity to each other, as shown in Figure 4.1, that are segmented as one object by the ACCEPT software. Therefore, the inclusion of such a parameter in EpCAM enriched samples would lead to an even higher underestimation of this population compared to the underestimation already introduced by cell clusters counted as one object. On the other hand, the removal of that parameter in case of blood samples with no enrichment (Figure 4.3), where it is very rare to find 2 or more leukocytes in close proximity, leads to the inclusion of artefacts and an overestimation of leukocytes. 
Table 4.1. ACCEPT gates used for the automated enumeration of leukocytes, leukocyte-derived EVs (ldEVs), platelets and red blood cells in blood A. with and B. without EpCAM enrichment.

\begin{tabular}{|c|c|c|c|c|c|}
\hline & & EpCAM enrichment & & No enrichment & \\
\hline \multirow[t]{10}{*}{ Leukocytes } & DAPI/ & Mean Intensity & $>30$ & Mean Intensity & $>30$ \\
\hline & Hoechst $^{\mathrm{a}}$ & Max Intensity & $>50$ & Max Intensity & $>50$ \\
\hline & & Size & $>16 \mu \mathrm{m}^{2}$ & Size & $>16 \mu \mathrm{m}^{2}$ \\
\hline & $\mathrm{CD} 45$ & Mean Intensity & $>30$ & Mean Intensity & $>30$ \\
\hline & & Max Intensity & $>50$ & Max Intensity & $>50$ \\
\hline & & & & Size & $\leq 400 \mu \mathrm{m}^{2}$ \\
\hline & CK & Standard Deviation & $\leq 5$ & $\mathrm{n} / \mathrm{a}^{\mathrm{b}}$ & \\
\hline & CD61 & $\mathrm{n} / \mathrm{a}^{\mathrm{b}}$ & & Standard Deviation & $\leq 5$ \\
\hline & CD235a & $\mathrm{n} / \mathrm{a}^{\mathrm{b}}$ & & Standard Deviation & $\leq 5$ \\
\hline & Extra channel & Standard Deviation & $\leq 5$ & Standard Deviation & $\leq 5$ \\
\hline \multirow[t]{11}{*}{ ldEVs } & DAPI/ & Standard Deviation & $\leq 5$ & Standard Deviation & $\leq 5$ \\
\hline & Hoechst ${ }^{\mathrm{a}}$ & & & & \\
\hline & $\mathrm{CD} 45$ & Mean Intensity & $>30$ & Mean Intensity & $>30$ \\
\hline & & Max Intensity & $>50$ & Max Intensity & $>50$ \\
\hline & & Perimeter & $>5$ pixels & Perimeter & $>5$ pixels \\
\hline & & Size & $\leq 150$ & Size & $\leq 150 \mu \mathrm{m}^{2}$ \\
\hline & & Eccentricity & $\leq 0.85$ & Eccentricity & $\leq 0.85$ \\
\hline & CK & Standard Deviation & $\leq 5$ & $\mathrm{n} / \mathrm{a}^{\mathrm{b}}$ & \\
\hline & CD61 & $\mathrm{n} / \mathrm{a}^{\mathrm{b}}$ & & Standard Deviation & $\leq 5$ \\
\hline & CD235a & $\mathrm{n} / \mathrm{a}^{\mathrm{b}}$ & & Standard Deviation & $\leq 5$ \\
\hline & Extra channel & Standard Deviation & $\leq 5$ & Standard Deviation & $\leq 5$ \\
\hline \multirow[t]{9}{*}{ Platelets } & $\mathrm{CD} 45$ & $\mathrm{n} / \mathrm{a}^{\mathrm{b}}$ & & Standard Deviation & $\leq 5$ \\
\hline & CD61 & & & Mean Intensity & $>30$ \\
\hline & & & & Max Intensity & $>50$ \\
\hline & & & & Perimeter & $>5$ pixels \\
\hline & & & & Size & $\leq 150 \mu \mathrm{m}^{2}$ \\
\hline & & & & Eccentricity & $\leq 0.85$ \\
\hline & CD235a & & & Standard Deviation & $\leq 5$ \\
\hline & & & & Standard Deviation & $\leq 5$ \\
\hline & Extra Channel & & & Standard Deviation & $\leq 5$ \\
\hline
\end{tabular}




\begin{tabular}{llll}
\hline & EpCAM enrichment & No enrichment & \\
\hline $\begin{array}{l}\text { Red blood } \\
\text { cells }\end{array}$ & Hoechst & $\mathrm{n} / \mathrm{a}^{\mathrm{b}}$ & Standard Deviation $\leq 5$ \\
& $\mathrm{CD} 45$ & & Standard Deviation $\leq 5$ \\
& $\mathrm{CD} 61$ & Standard Deviation $\leq 5$ \\
& CD235a & Mean Intensity & $>30$ \\
& & Max Intensity & $>50$ \\
& & Perimeter & $>5$ pixels \\
& & & Standard Deviation $\leq 5$ \\
\hline
\end{tabular}

${ }^{a}$ DAPI was used in EpCAM enriched blood and Hoechst in blood without pre-enrichment, ${ }^{b} \mathrm{n} / \mathrm{a}$ : not applicable.

For the acquisition of the fluorescence images of cells and EVs in blood without any pre-enrichment, the focus was set on 4 points distributed throughout each well to achieve optimal visualization of the cells and the surface was afterwards automatically scanned. Since the objects were in suspension and not attached on a surface, most ldEVs and platelets, were out-of-focus with their blurring pattern influencing their perceived size, that seems much larger in the respective fluorescence images than it actually is. Hence, the size of ldEVs cannot be accurately derived using the immunofluorescence images (Figure 4.2). In case of EpCAM enriched samples, EVs are aligned on the same focal plane as the cells due to the design of the Cellsearch magnest that results in an homogeneous cell distribution along the applied magnetic field ${ }^{24}$. Even in that case, however, the use of immunofluorescence images can lead to erroneous conclusions about the size of EVs. That is even more profound when low magnification objectives are used in the fluorescence microscopes as in our case (10x/0.45 NA) limiting the determination of the size of EVs with confidence since each pixel of the acquired images corresponds to $0.64 \times 0.64 \mu \mathrm{m}^{2}$. More examples of correlated bright field and immunofluorescence images of ldEVs in whole blood samples can be found in Supplementary Figure S4.2.

\subsubsection{Absolute and relative frequencies of leukocytes and ldEVs in 7.5 $\mathrm{mL}$ of EpCAM enriched blood of healthy individuals and metastatic cancer patients.}

The numbers of ldEVs and leukocytes in EpCAM enriched $7.5 \mathrm{ml}$ blood samples of 25 healthy individuals, 25 metastatic prostate, 25 colorectal and 25 non-small cell lung cancer patients were determined and are presented in box plots (Figure 4.4). In addition, the number of ldEVs and leukocytes present in $0.02 \mu \mathrm{l}$ of blood of 10 healthy individuals with no enrichment was determined and extrapolated to $7.5 \mathrm{~mL}$ of blood for comparison (Figure 4.4). As it was expected, the leukocyte and ldEV frequencies are significantly depleted in the EpCAM enriched blood samples of individuals, since EpCAM is an epithelial marker that is not expected to be expressed on the surface of leukocytes and ldEVs; therefore leukocytes and ldEVs are not positively selected by 
the EpCAM ferrofluid. For each sample (with or without EpCAM enrichment), the relative frequencies of ldEVs over leukocytes were calculated. In the blood of healthy individuals with no enrichment, I ldEV was detected for every 3 leukocytes. In the EpCAM enriched blood of both healthy individuals and metastatic cancer patients, the relative frequencies of IdEVs over leukocytes was found to be approximately half, with $1 \mathrm{ldEV}$ being detected for every 5 (in case of samples from healthy individuals, prostate cancer and non-small cell lung cancer patients) to 6 (in case of samples from colorectal cancer patients) leukocytes. The presence of ldEVs in higher relative frequencies in whole blood of individuals compared to EpCAM enriched samples can be attributed to three reasons. Firstly, the blood samples are centrifuged at $800 x \mathrm{x}$ for $10 \mathrm{~min}$ and the plasma fraction containing the majority of extracellular vesicles is not processed by the Cellsearch system implying that half of the ldEVs detected in the blood samples without EpCAM enrichment end up in the plasma fraction. Secondly, the Fcy receptors of leukocytes and ldEVs are expected to bind to the heavy chains rather than the antigen binding sites of the antibodies against EpCAM that are conjugated to the ferrofluid. As ldEVs have fewer receptors due to their smaller surface, their carryover in the EPCAM enriched sample should be lower than leukocytes. The third reason for that observation might be the lower CD45 signal of leukocytes and ldEVs in the images of the CellTracks Analyzer II compared to the imaging setup used in case of the blood samples without EpCAM enrichment; the CellTracks Analyzer II uses a mercury arc lamp that results in a suboptimal excitation of the APC-conjugated antibody against CD45 in contrast to the other imaging setup that uses a LED light source. In combination with the smaller size of ldEVs, this could lead to ldEVs with a CD45 signal close to the background intensity not being considered as true events; thereby underestimating the $\mathrm{IEV}$ frequencies. In any case, the relative frequencies of ldEVs to leukocytes in blood samples with and without EPCAM enrichment are of a similar order of magnitude (1:3 and 1:5 respectively) supporting their pre-existence in blood circulation of individuals and rejecting a possible hypothesis of their formation during the Cellsearch procedure.

\subsubsection{Reproducibility of measurements by fluorescence imaging and correlation with frequencies of blood cells by hematology analyzer}

The technical variability ( $\mathrm{N}=4-6$ technical replicates) of measuring cell populations in $0.02 \mu \mathrm{l}$ of blood of healthy individuals without any pre-enrichment was assessed by performing 4-6 replicates of $0.02 \mu \mathrm{l}$ blood from 10 healthy individuals. An average standard error of 25,18 and $23 \%$ was obtained for respectively leukocytes, red blood cells and platelets. The respective standard error for ldEVs from the technical replicates was found to be $50 \%$ because of the very low frequency of ldEVs in $0.02 \mu \mathrm{l}$ of blood processed, that was found to be $18 \pm 5$ (mean value \pm SD). We expect that processing larger blood volumes would lead to lower technical variations. 


\section{Absolute frequencies of IdEVs and leukocytes}

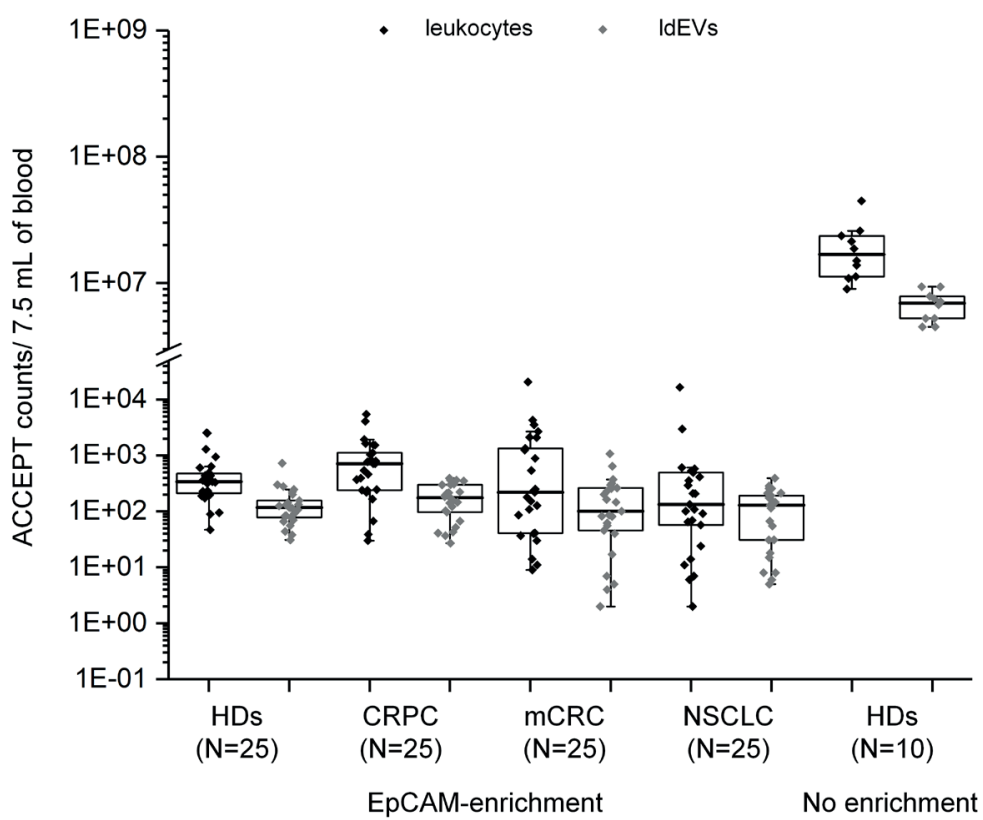

Figure 4.4. Absolute frequencies of leukocytes and large ldEVs in $7.5 \mathrm{~mL}$ of blood of individuals with and without EpCAM enrichment. The interquartile range of absolute frequencies of leukocytes (data in black dots) and ldEVs (data in grey dots) in whole blood of 10 healthy individuals and EpCAM enriched blood samples of 25 healthy individuals (HDs) and 75 EpCAM enriched blood samples of metastatic prostate (CRPC), colorectal (MCRC) and non-small cell lung (NSCLC) cancer patients are shown in box plots. Whiskers indicate max and min values as estimated by Q3+1.5* IQR and Q11.5"IQR respectively. Each dot in case of blood of healthy individuals without EpCAM enrichment corresponds to the mean values of 4-6 technical replicates.

The averaged counts of the blood cell classes as estimated by the immunofluorescence imaging were extrapolated to $1 \mu \mathrm{l}$ of blood and compared to the respective frequencies obtained by the hematology analyzer. The measurements of the fluorescence imaging were moderately correlated $\left(\mathrm{R}^{2} \approx 0.7\right)$ with the counts from the hematology analyzer as shown in Figure 4.5. However, all cell populations were underestimated by the fluorescence imaging approach when compared to the hematology analyzer. That can be justified by the low CD45 expression of neutrophils that comprise $60-70 \%$ of the whole leukocyte population, the overlap and aggregation of red blood cells (Supplementary Figure S4.1) that are considered as one when enumerated using the ACCEPT software and the detection limit of fluorescence imaging in case of smaller size platelets. 
Correlation of hematology analyzer and fluorescence imaging outputs
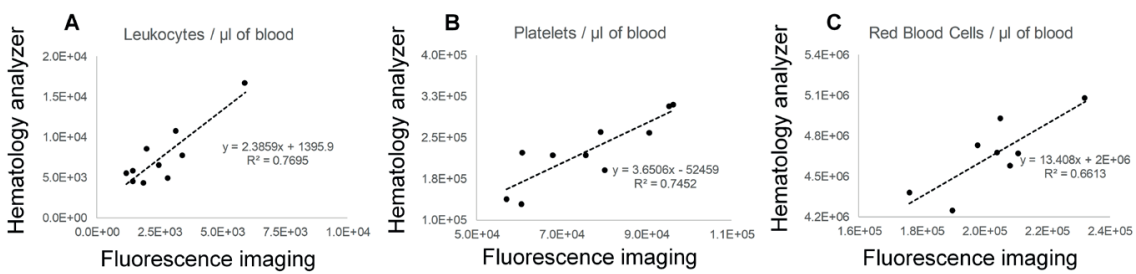

Figure 4.5. Scatter plots of leukocyte (Panel A), platelet (Panel B) and red blood cell frequencies (Panel C) in $1 \mu l$ of whole blood of 10 healthy individuals as estimated by fluorescence imaging and ACCEPT enumeration ( $\mathrm{x}$-axis) and by hematology analyzer (y-axis). Correlation between the measurements of the 2 techniques was found as indicated by the $\mathrm{R}^{2}$. The mean counts of each population of $4-6$ technical replicates were used in case of the fluorescence imaging approach.

\subsection{Discussion}

The Extracellular Vesicle field has focused so far on the biogenesis and functions of EVs with a size smaller than $1 \mu \mathrm{m}$ secreted by various cells ${ }^{28}$, including platelets ${ }^{29}$, neutrophils ${ }^{30}$, T and B lymphocytes ${ }^{31}$, red blood cells ${ }^{32}$, endothelial cells ${ }^{33,34}$ and tumor cells ${ }^{35}$. However, the existing literature on the formation and frequencies of EVs larger than $1 \mu \mathrm{m}$ in healthy individuals is very sparse, as they are considered to be apoptotic bodies, and as such not actively contributing in the intercellular communication. Nevertheless, recent findings in the cancer field, shows the promising potential of large tdEVs as their load associates with clinical outcome in metastatic cancer patients ${ }^{18,27}$ and their molecular cargo represents the mutational status of the tumor ${ }^{9}$. Our previous research on scanning electron microscopy imaging of CellSearch cartridges of castration-resistant prostate cancer patient samples after EpCAM enrichment ${ }^{36}$ and the development of the open-source ACCEPT software for the automated enumeration of all fluorescently labeled objects from image data sets led to our first observations of the presence of DAPI-, CD45+, CK- objects ${ }^{21}$. We baptized these objects leukocytederived Extracellular Vesicles (ldEVs) and investigated their presence in digitally stored fluorescence images of CellSearch cartridges. The ldEVs had a similar size range to our previously reported DAPI-, CD45-, CK+ tumor-derived Extracellular Vesicles (tdEVs), that were detected after EpCAM enrichment in metastatic cancer patients ${ }^{18,19}$. Their detection raised questions regarding the pre-existence of these large ldEVs and tdEVs in blood circulation of individuals or their formation as fragmentation by-products of leukocytes and CTCs respectively during the CellSearch procedure.

Therefore, we decided to address the question of whether these EVs pre-exist in blood circulation of individuals. Towards that direction, we enumerated ldEVs in blood samples of 10 healthy individuals without any pre-analytical or pre-enrichment steps 
and compared the frequencies of ldEVs and leukocytes in whole blood to the frequency in EpCAM enriched blood samples of 25 healthy individuals and 75 metastatic cancer patients. ldEVs and leukocytes were detected in a ratio of 1:3 in blood of healthy individuals without any pre-enrichment and in 1:5 to 1:6 in EpCAM enriched blood of healthy individuals and metastatic cancer patients (Figure 4.4) supporting the pre-existence of these ldEVs in blood circulation instead of their formation during the EPCAM enrichment. The lower relative frequencies of ldEVs to leukocytes in the EpCAM enriched blood samples compared to the samples without EpCAM enrichment can be mainly explained by the blood fraction that is processed by the Cellsearch system: blood samples are centrifuged at $800 \mathrm{xg}$ for $10 \mathrm{~min}$ and the plasma fraction is discarded and not processed by the system. Using that centrifugation force, only EVs with a diameter above $1 \mu \mathrm{m}$ will be in the blood fraction and will have the chance to come into contact with the EpCAM ferrofluid ${ }^{37}$. The measurements in the blood of healthy donors without EpCAM enrichment were reproducible, as confirmed by the standard deviations of the technical replicates, with a mean \pm SD of $900 \pm 254$ ldEVs in $1 \mu \mathrm{l}$ of blood of healthy individuals. These results do not deviate a lot from the previously reported ldEV frequencies (median value: 356, interquartile range: 268 - 529) of Simak et al. in the plasma of healthy donors; ldEVs were larger than $200 \mathrm{~nm}$ and were defined as CD45+, CD105-, CD235a- by flow cytometry ${ }^{38}$. The use of solely one specific but weakly expressed inclusion marker, namely CD45 to define them using either approaches, results in the underestimation of the whole circulating IdEV population; a point stressed out also by Lacroix et al. ${ }^{39}$. Further investigation of ldEVs in terms of their size distribution and surface marker expression using established techniques in the EV field, such as nanoparticle tracking analysis, electron microscopy, flow cytometry and surface plasmon resonance imaging, would lead to their better profiling ${ }^{10,40,41}$. Eventually, a similar test to hematology analyzer having as an output the EV populations (of platelet, erythrocyte, leukocyte, endothelial and epithelial origin) in biofluids of individuals could serve as an important diagnostic tool in clinical practice, since EVs have been associated with numerous pathophysiological conditions, such as thrombogenicity, inflammation, angiogenesis and cancer ${ }^{28,42-45}$.

Our study has several limitations. Although the extrapolated counts of leukocytes, platelets and red blood cells per $\mu \mathrm{L}$ of blood correlated to the respective values of the hematology analyzer (Figure 4.5), the detected frequencies of all cell populations were consistently lower as compared to the respective ones measured by the hematology analyzer. The particularly lower detection of red blood cells can be explained by the overlap or/and aggregation of more than 1 red blood cells segmented as one object by the open-source ACCEPT software and the large range in the distribution of the fluorescence intensity of CD235-APC in the fluorescence images (Supplementary Figure S4.1, Panel A) through which part of the red blood cells fall outside the applied gate. The underestimation of leukocytes can be explained by the lower expression of CD45 by the granulocytes that consist to $60-70 \%$ of white blood cells. The detection 
of the smaller platelet population and their secreted EVs is limited by our approach, because of the use of a $0.45 \mathrm{NA}$ objective that results in a resolution of $0.64 \mu \mathrm{m} / \mathrm{pixel}$. The abundance and high signal of red blood cells prevented us also from the detection and enumeration of low signal-to-background ratio red blood cell- derived EVs. Importantly, the Hoechst 33342 labelled nucleic acids in platelets could be detected by flow cytometry but not with our microscopy set-up. This observation implies that the zero DAPI signal detected with ACCEPT inside our previously reported tdEVs does not rule out the presence of nucleic acids within them. This is an important finding encouraging the further characterization of tdEVs that are immunomagnetically isolated based on their EpCAM expression ${ }^{18,19,27}$. That would come into agreement with the findings of Vagner et al. that the DNA cargo of large EVs reflect the genotype of prostate cancer patients ${ }^{9}$. The use of a membrane permeable dye, binding to both DNA and RNA, with a higher sensitivity, like SYTO13 ${ }^{46}$ could also facilitate the detection of nucleic acids within the isolated EVs.

Interestingly, platelets have a similar size to ldEVs based on the immunofluorescene images of CD61 and CD45 respectively with a minimum area of the detected objects being $9 \mu \mathrm{m}^{2}$ based on our observations from the ACCEPT scatter plots. That area corresponds to a circular object of an approximate radius of $1.7 \mu \mathrm{m}$. However, it was not possible to confirm the size of ldEVs from the respective bright field images, because oppositely to platelets, the contrast between the background and ldEV intensity was inadequate to detect them (Figure 4.2 and Supplementary Figure S4.2) suggesting that their physical properties (absorption coefficient, scattering coefficient, scattering anisotropy, refractive index) differ from the ones of platelets.

It is worth mentioning that ldEVs were found in 5-6 lower frequencies compared to leukocytes in EpCAM enriched samples, whereas tdEVs in our previous studies were detected in 10-20 times higher frequencies compared to CTCs ${ }^{18}$. That observation can be explained after taking into consideration some technical and biological facts. From a technical perspective, our study was limited by the resolution of a 10x/0.45 NA objective fluorescence microscope, implying that only the larger EVs with a larger than $1 \mu \mathrm{m}$ diameter or the ones with a high expression of inclusion markers could be detected. CK is the inclusion marker used for the detection of tdEVs, whereas the detection of ldEVs is accomplished by the inclusion of CD45. Since the CK expression is intracellular and proportional to the volume instead of the surface as in the case of the CD45 expression of ldEVs, CK will be easier to detect in smaller tdEVs than CD45 in similar size ldEVs. Consequently, the CD45 expression may be present in more particles in blood samples but not exceeding the detection limit to be considered positive. Further characterization of the size distribution and the surface marker expression profile could elaborate on the detection limits of our technique. From a biological perspective, tdEVs could be found in higher frequencies either because of the increased apoptosis and fragmentation of CTCs in the blood circulation or because 
of different EV secretion pattern between normal and cancerous cells. Regarding the first hypothesis, the lifespan of neutrophils is around $24 \mathrm{~h}{ }^{47}$, whereas the circulation lifetime of CTCs has been estimated to be 1 to $2.4 \mathrm{~h}^{48}$; that however, does not imply that CTCs are fragmented and cleared by the blood. On the contrary, in vivo animal studies showed the trap of more than $80 \%$ of viable CTCs by the liver and lung, that serve as "filter" organs, and the survival of CTCs for prolonged time in a dormant state ${ }^{49,50}$. The survival of CTCs in the blood stream is further supported by studies on their mechanical phenotype. Atomic force microscopy studies on cell lines, suggest that cells with increased metastatic potential are more deformable (as expressed by Young's modulus), compared to less metastatic or non-malignant cells ${ }^{51,52}$. These results were further confirmed on clinical samples from pleural effusions, where metastatic cells had lower stiffness compared to benign cells from the same effusions and leukocytes ${ }^{53,54}$. Interestingly, Sun et al. demonstrated that deformable cancer cells engulf neighboring ones with higher stiffness via entosis further encouraging the increased survivorship of CTCs ${ }^{55}$. Regarding the second hypothesis of different EV secretion pattern of CTCs and leukocytes, it is well known that cancer cells have reprogrammed metabolism and acquired traits that promote their survival and growth ${ }^{56,57}$. Recent findings of independent research groups converge into the survival of tumor cells regardless their phenotypic characteristics of a possible apoptosis, such as caspases activation, amoeboid phenotype and membrane blebbing ${ }^{58,59}$. Instead of undergoing apoptosis, cells with such traits have a more tumorigenic and invasive phenotype ${ }^{60,61}$. Hence, tumor cells may actively secrete EVs similar in size to apoptotic bodies, but without special receptors on their surface to be recognized and ingested by macrophages for their clearance as in case of healthy cells (eg white blood cells).

In conclusion, the relative frequencies of large (above $1 \mu \mathrm{m}$ ) leukocyte-derived Extracellular Vesicles (ldEVs) to leukocytes are similar in EpCAM enriched blood samples of healthy individuals and cancer patients (1:6 to 1:5) as in blood of healthy individuals without EpCAM enrichment (1:3), implying their pre-existence in blood circulation rather than their formation from activated or apoptotic leukocytes using the CellSearch system. Furthermore, the Hoechst signal of platelets could not be detected using a similar fluorescence microscope as the CellTracks Analyzer II. These two findings have important implications for our previously reported tumor-derived Extracellular Vesicles (tdEVs), that were immunomagnetically co-isolated with CTCs based on their EpCAM expression from metastatic cancer patients ${ }^{19}$. Firstly, tdEVs are most likely not a result of CTC fragmentation during the CellSearch procedure and secondly, the presence of undetectable nucleic acids within IdEVs and tdEVs should not be excluded but instead further investigated. No conclusions can be drawn in regards to the smaller $\mathrm{ldEV}$ population, namely exosomes, since they are not expected to be detected with our imaging setup. Last but not least, our results do not allow for comparison of ldEVs between healthy individuals and cancer patients, since the available image data sets of patients corresponded to only EpCAM enriched samples. 


\section{Acknowledgments}

The authors acknowledge the Experimental Centre for Technical Medicine (ECTM) of the University of Twente for providing us blood samples of healthy individuals. We would also like to thank all the patients and healthy individuals, the blood samples of whom were used for the accomplishment of the present study. Special acknowledgments to C. Breukers and J. Weersink for their technical support with the inverted fluorescence microscope whenever needed. 


\section{References}

1. van Niel G, D'Angelo G, Raposo G. Shedding light on the cell biology of extracellular vesicles. Nat Rev Mol Cell Biol 2018; 19(4): 213-228; doi 10.1038/nrm.2017.125.

2. Fais S, O'Driscoll L, Borras FE, Buzas E, Camussi G, Cappello F et al. Evidence-based clinical use of nanoscale extracellular vesicles in nanomedicine. ACS nano 2016; 10(4): 3886-3899.

3. Yanez-Mo M, Siljander PR, Andreu Z, Zavec AB, Borras FE, Buzas EI et al. Biological properties of extracellular vesicles and their physiological functions. J Extracell Vesicles 2015; 4: 27066; doi 10.3402/jev.v4.27066.

4. Jia S, Zhang R, Li Z, Li J. Clinical and biological significance of circulating tumor cells, circulating tumor DNA, and exosomes as biomarkers in colorectal cancer. Oncotarget 2017; 8(33): 55632-55645.

5. Krug AK, Enderle D, Karlovich C, Priewasser T, Bentink S, Spiel A et al. Improved EGFR mutation detection using combined exosomal RNA and circulating tumor DNA in NSCLC patient plasma. Annals of oncology : official journal of the European Society for Medical Oncology 2018; 29(10): 2143.

6. Costa-Silva B, Aiello NM, Ocean AJ, Singh S, Zhang H, Thakur BK et al. Pancreatic cancer exosomes initiate pre-metastatic niche formation in the liver. Nat Cell Biol 2015; 17(6): 816-826; doi 10.1038/ncb3169.

7. Hoshino A, Costa-Silva B, Shen TL, Rodrigues G, Hashimoto A, Tesic Mark M et al. Tumour exosome integrins determine organotropic metastasis. Nature 2015; 527(7578): 329-335; doi 10.1038/nature15756.

8. Kosaka N, Yoshioka Y, Fujita Y, Ochiya T. Versatile roles of extracellular vesicles in cancer. J Clin Invest 2016; 126(4): 1163-1172; doi 10.1172/JCI81130.

9. Vagner T, Spinelli C, Minciacchi VR, Balaj L, Zandian M, Conley A et al. Large extracellular vesicles carry most of the tumour DNA circulating in prostate cancer patient plasma. Journal of extracellular vesicles 2018; 7(1): 1505403.

10. Ricklefs FL, Maire CL, Reimer R, Duhrsen L, Kolbe K, Holz M et al. Imaging flow cytometry facilitates multiparametric characterization of extracellular vesicles in malignant brain tumours. J Extracell Vesicles 2019; 8(1): 1588555; doi 10.1080/20013078.2019.1588555.

11. Konig L, Kasimir-Bauer S, Bittner AK, Hoffmann O, Wagner B, Santos Manvailer LF et al. Elevated levels of extracellular vesicles are associated with therapy failure and disease progression in breast cancer patients undergoing neoadjuvant chemotherapy. Oncoimmunology 2017; 7(1): e1376153; doi 10.1080/2162402X.2017.1376153.

12. Kanwar SS, Dunlay CJ, Simeone DM, Nagrath S. Microfluidic device (ExoChip) for on-chip isolation, quantification and characterization of circulating exosomes. Lab Chip 2014; 14(11): 1891-1900; doi 10.1039/c4lcoo136b.

13. van der Pol E, Boing AN, Harrison P, Sturk A, Nieuwland R. Classification, functions, and clinical relevance of extracellular vesicles. Pharmacol Rev 2012; 64(3): 676-705; doi 10.1124/ pr.112.005983. 
14. Slomka A, Urban SK, Lukacs-Kornek V, Zekanowska E, Kornek M. Large Extracellular Vesicles: Have We Found the Holy Grail of Inflammation? Front Immunol 2018; 9: 2723; doi 10.3389/fimmu.2018.02723.

15. Vagner T, Spinelli C, Minciacchi VR, Balaj L, Zandian M, Conley A et al. Large extracellular vesicles carry most of the tumour DNA circulating in prostate cancer patient plasma. $J$ Extracell Vesicles 2018; 7(1): 1505403; doi 10.1080/20013078.2018.1505403.

16. Padda RS, Deng FK, Brett SI, Biggs CN, Durfee PN, Brinker CJ et al. Nanoscale flow cytometry to distinguish subpopulations of prostate extracellular vesicles in patient plasma. Prostate 2019; 79(6): 592-603; doi 10.1002/pros.23764.

17. Reategui E, van der Vos KE, Lai CP, Zeinali M, Atai NA, Aldikacti B et al. Engineered nanointerfaces for microfluidic isolation and molecular profiling of tumor-specific extracellular vesicles. Nat Commun 2018; 9(1): 175; doi 10.1038/s41467-017-02261-1.

18. Nanou A, Coumans FAW, van Dalum G, Zeune LL, Dolling D, Onstenk W et al. Circulating tumor cells, tumor-derived extracellular vesicles and plasma cytokeratins in castrationresistant prostate cancer patients. Oncotarget 2018; 9(27): 19283-19293; doi 10.18632/ oncotarget.25019.

19. Nanou A., Zeune L.L., de Wit S., Miller C.M., Punt C.J.A., Groen H.J.M. et al. Tumor-derived extracellular vesicles in blood of metastatic breast, colorectal, prostate and non-small cell lung cancer patients associate with worse survival. Paper presented at: AACR Annual Meeting2019.

20. Zeune L, van Dalum G, Terstappen LWMM, van Gils SA, Brune C. Multiscale Segmentation via Bregman Distances and Nonlinear Spectral Analysis. Siam I Imaging Sci 2017; 10(1): 111-146; doi $10.1137 / 16 \mathrm{~m} 1074503$.

21. Zeune L. Automated CTC Classification, Enumeration and Pheno Typing: Where Math meets Biology [PhD dissertation]. Enschede: Medical Cell BioPhysics, University of Twente; 2019.

22. Coumans F, Terstappen L. Detection and Characterization of Circulating Tumor Cells by the CellSearch Approach. Methods in molecular biology (Clifton, NJ) 2015; 1347: 263-278.

23. Allard WJ, Matera J, Miller MC, Repollet M, Connelly MC, Rao C et al. Tumor cells circulate in the peripheral blood of all major carcinomas but not in healthy subjects or patients with nonmalignant diseases. Clin Cancer Res 2004; 10(20): 6897-6904; doi 10.1158/1078-0432.CCR04-0378.

24. Tibbe AG, de Grooth BG, Greve J, Dolan GJ, Rao C, Terstappen LW. Magnetic field design for selecting and aligning immunomagnetic labeled cells. Cytometry 2002; 47(3): 163-172.

25. de Wit S, Zeune LL, Hiltermann TJN, Groen HJM, Dalum Gv, Terstappen LWMM. Classification of Cells in CTC-Enriched Samples by Advanced Image Analysis. Cancers 2018; 10(10).

26. Zeune LL, de Wit S, Berghuis AMS, MJ IJ, Terstappen L, Brune C. How to Agree on a CTC: Evaluating the Consensus in Circulating Tumor Cell Scoring. Cytometry A 2018; 93(12): 12021206; doi 10.1002/cyto.a.23576.

27. de Wit S, Rossi E, Weber S, Tamminga M, Manicone M, Swennenhuis JF et al. Single tube liquid biopsy for advanced non-small cell lung cancer. Int J Cancer 2018; doi 10.1002/ijc.32056. 
28. van der Pol E, Boing AN, Harrison P, Sturk A, Nieuwland R. Classification, functions, and clinical relevance of extracellular vesicles. Pharmacological reviews 2012; 64(3): 676-705.

29. Heijnen HF, Schiel AE, Fijnheer R, Geuze HJ, Sixma JJ. Activated platelets release two types of membrane vesicles: microvesicles by surface shedding and exosomes derived from exocytosis of multivesicular bodies and alpha-granules. Blood 1999; 94(11): 3791-3799.

30. Pitanga TN, de Aragao Franca L, Rocha VC, Meirelles T, Borges VM, Goncalves MS et al. Neutrophil-derived microparticles induce myeloperoxidase-mediated damage of vascular endothelial cells. BMC Cell Biol 2014; 15: 21; doi 10.1186/1471-2121-15-21.

31. Baka Z, Senolt L, Vencovsky J, Mann H, Simon PS, Kittel A et al. Increased serum concentration of immune cell derived microparticles in polymyositis/dermatomyositis. Immunol Lett 2010; 128(2): 124-130; doi 10.1016/j.imlet.2009.12.018.

32. Canellini G, Rubin O, Delobel J, Crettaz D, Lion N, Tissot JD. Red blood cell microparticles and blood group antigens: an analysis by flow cytometry. Blood Transfus 2012; 10 Suppl 2: s39-45; doi 10.2450/2012.007S.

33. Wheway J, Latham SL, Combes V, Grau GER. Endothelial microparticles interact with and support the proliferation of $\mathrm{T}$ cells. Journal of immunology (Baltimore, $M d$ : 1950) 2014; 193(7): 3378-3387.

34. Deregibus MC, Cantaluppi V, Calogero R, Lo Iacono M, Tetta C, Biancone L et al. Endothelial progenitor cell derived microvesicles activate an angiogenic program in endothelial cells by a horizontal transfer of mRNA. Blood 2007; 110(7): 2440-2448.

35. Ruhen O, Meehan K. Tumor-Derived Extracellular Vesicles as a Novel Source of Protein Biomarkers for Cancer Diagnosis and Monitoring. Proteomics 2019; 19(1-2): e1800155.

36. Nanou A, Crespo M, Flohr P, De Bono JS, Terstappen L. Scanning Electron Microscopy of Circulating Tumor Cells and Tumor-Derived Extracellular Vesicles. Cancers (Basel) 2018; 10(11); doi 10.3390/cancers10110416.

37. Rikkert LG, van der Pol E, van Leeuwen TG, Nieuwland R, Coumans FAW. Centrifugation affects the purity of liquid biopsy-based tumor biomarkers. Cytometry A 2018; 93(12): 12071212; doi 10.1002/cyto.a.23641.

38. Simak J, Holada K, Risitano AM, Zivny JH, Young NS, Vostal JG. Elevated circulating endothelial membrane microparticles in paroxysmal nocturnal haemoglobinuria. British journal of haematology 2004; 125(6): 804-813.

39. Lacroix R, Robert S, Poncelet P, Dignat-George F. Overcoming limitations of microparticle measurement by flow cytometry. Semin Thromb Hemost 2010; 36(8): 807-818; doi 10.1055/s0030-1267034.

40. van der Pol E, Coumans FA, Grootemaat AE, Gardiner C, Sargent IL, Harrison P et al. Particle size distribution of exosomes and microvesicles determined by transmission electron microscopy, flow cytometry, nanoparticle tracking analysis, and resistive pulse sensing. J Thromb Haemost 2014; 12(7): 1182-1192; doi 10.1111/jth.12602.

41. Gool EL, Stojanovic I, Schasfoort RBM, Sturk A, van Leeuwen TG, Nieuwland R et al. Surface Plasmon Resonance is an Analytically Sensitive Method for Antigen Profiling of Extracellular Vesicles. Clin Chem 2017; 63(10): 1633-1641; doi 10.1373/clinchem.2016.271049. 
42. Halim ATA, Ariffin NAFM, Azlan M. Review: the Multiple Roles of Monocytic Microparticles. Inflammation 2016; 39(4): 1277-1284.

43. Tissot J-D, Canellini G, Rubin O, Angelillo-Scherrer A, Delobel J, Prudent M et al. Blood microvesicles: from proteomics to physiology. Translational Proteomics 2013; 1(1):38-52.

44. Burnier L, Fontana P, Kwak BR, Angelillo-Scherrer A. Cell-derived microparticles in haemostasis and vascular medicine. Thromb Haemost 2009; 101(3): 439-451.

45. Julich-Haertel H, Urban SK, Krawczyk M, Willms A, Jankowski K, Patkowski W et al. Cancerassociated circulating large extracellular vesicles in cholangiocarcinoma and hepatocellular carcinoma. Journal of hepatology 2017; 67(2): 282-292.

46. Ullal AJ, Pisetsky DS, Reich CF, 3rd. Use of SYTO 13, a fluorescent dye binding nucleic acids, for the detection of microparticles in in vitro systems. Cytometry Part A : the journal of the International Society for Analytical Cytology 2010; 77(3): 294-301.

47. McCracken JM, Allen LA. Regulation of human neutrophil apoptosis and lifespan in health and disease. J Cell Death 2014; 7: 15-23; doi 10.4137/JCD.S11038.

48. Meng S, Tripathy D, Frenkel EP, Shete S, Naftalis EZ, Huth JF et al. Circulating tumor cells in patients with breast cancer dormancy. Clin Cancer Res 2004;10(24): 8152-8162; doi 10.1158/10780432.CCR-04-1110.

49. Luzzi KJ, MacDonald IC, Schmidt EE, Kerkvliet N, Morris VL, Chambers AF et al. Multistep nature of metastatic inefficiency: dormancy of solitary cells after successful extravasation and limited survival of early micrometastases. Am J Pathol 1998; 153(3): 865-873; doi 10.1016/ So002-9440(10)65628-3.

50. Cameron MD, Schmidt EE, Kerkvliet N, Nadkarni KV, Morris VL, Groom AC et al. Temporal progression of metastasis in lung: cell survival, dormancy, and location dependence of metastatic inefficiency. Cancer Res 2000; 60(9): 2541-2546.

51. Li QS, Lee GYH, Ong CN, Lim CT. AFM indentation study of breast cancer cells. Biochemical and biophysical research communications 2008; 374(4): 609-613.

52. Zhang W, Kai K, Choi DS, Iwamoto T, Nguyen YH, Wong $\mathrm{H}$ et al. Microfluidics separation reveals the stem-cell-like deformability of tumor-initiating cells. Proceedings of the National Academy of Sciences of the United States of America 2012; 109(46): 18707-18712.

53. Cross SE, Jin Y-S, Rao J, Gimzewski JK. Nanomechanical analysis of cells from cancer patients. Nat Nanotechnol 2007; 2(12): 780-783.

54. Gossett DR, Tse HTK, Lee SA, Ying Y, Lindgren AG, Yang OO et al. Hydrodynamic stretching of single cells for large population mechanical phenotyping. Proceedings of the National Academy of Sciences of the United States of America 2012; 109(20): 7630-7635.

55. Sun Q Luo T, Ren Y, Florey O, Shirasawa S, Sasazuki T et al. Competition between human cells by entosis. Cell Res 2014; 24(11): 1299-1310.

56. Hanahan D, Weinberg RA. Hallmarks of cancer: the next generation. Cell 2011; 144(5): 646-674

57. Hanahan D, Weinberg RA. The hallmarks of cancer. Cell 2000; 100(1): 57-70.

58. Jinesh GG, Choi W, Shah JB, Lee EK, Willis DL, Kamat AM. Blebbishields, the emergency program for cancer stem cells: sphere formation and tumorigenesis after apoptosis. Cell Death Differ 2013; 20(3): 382-395. 
59. Di Vizio D, Morello M, Dudley AC, Schow PW, Adam RM, Morley S et al. Large oncosomes in human prostate cancer tissues and in the circulation of mice with metastatic disease. Am J Pathol 2012; 181(5): 1573-1584; doi 10.1016/j.ajpath.2012.07.030.

60. Di Vizio D, Kim J, Hager MH, Morello M, Yang W, Lafargue CJ et al. Oncosome formation in prostate cancer: association with a region of frequent chromosomal deletion in metastatic disease. Cancer Res 2009; 69(13): 5601-5609; doi 10.1158/0008-5472.CAN-08-3860.

61. Reis-Sobreiro M, Chen JF, Novitskaya T, You S, Morley S, Steadman K et al. Emerin Deregulation Links Nuclear Shape Instability to Metastatic Potential. Cancer Res 2018; 78(21): 6086-6097; doi 10.1158/0008-5472.CAN-18-0608. 


\section{Supplementary Data}

A. $10 \times / 0.45$ NA objective

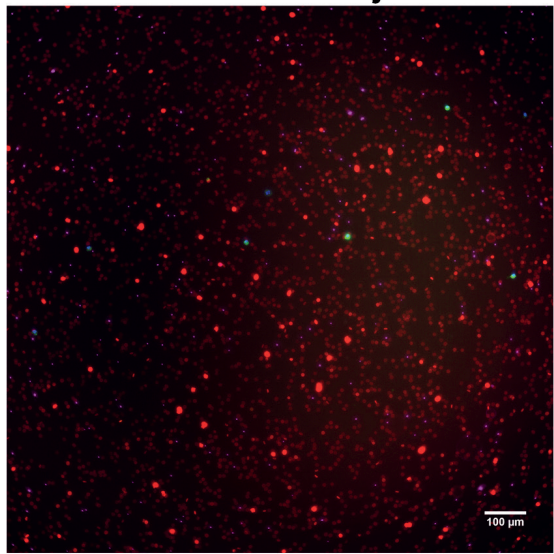

Hoechst, CD235a, CD61, CD45
B. $60 \times / 0.7$ NA objective

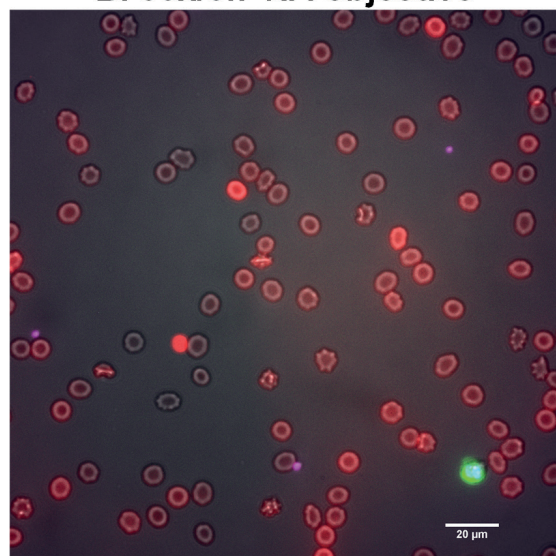

Hoechst, CD235a, CD61, CD45, brightfield

Supplementary Figure S4.1. Examples of composite immunofluorescence images of blood samples without EpCAM enrichment, obtained with an inverted scanning fluorescence microscope using a $10 \times / 0.45$ NA (Panel A) and a 60x/0.7 NA objective (Panel B). The images that were obtained with the 10x objective were used as an input in the open-source ACCEPT software for the automated enumeration of the different cell and EV populations. 


\section{Leukocyte-derived EVs in whole blood}

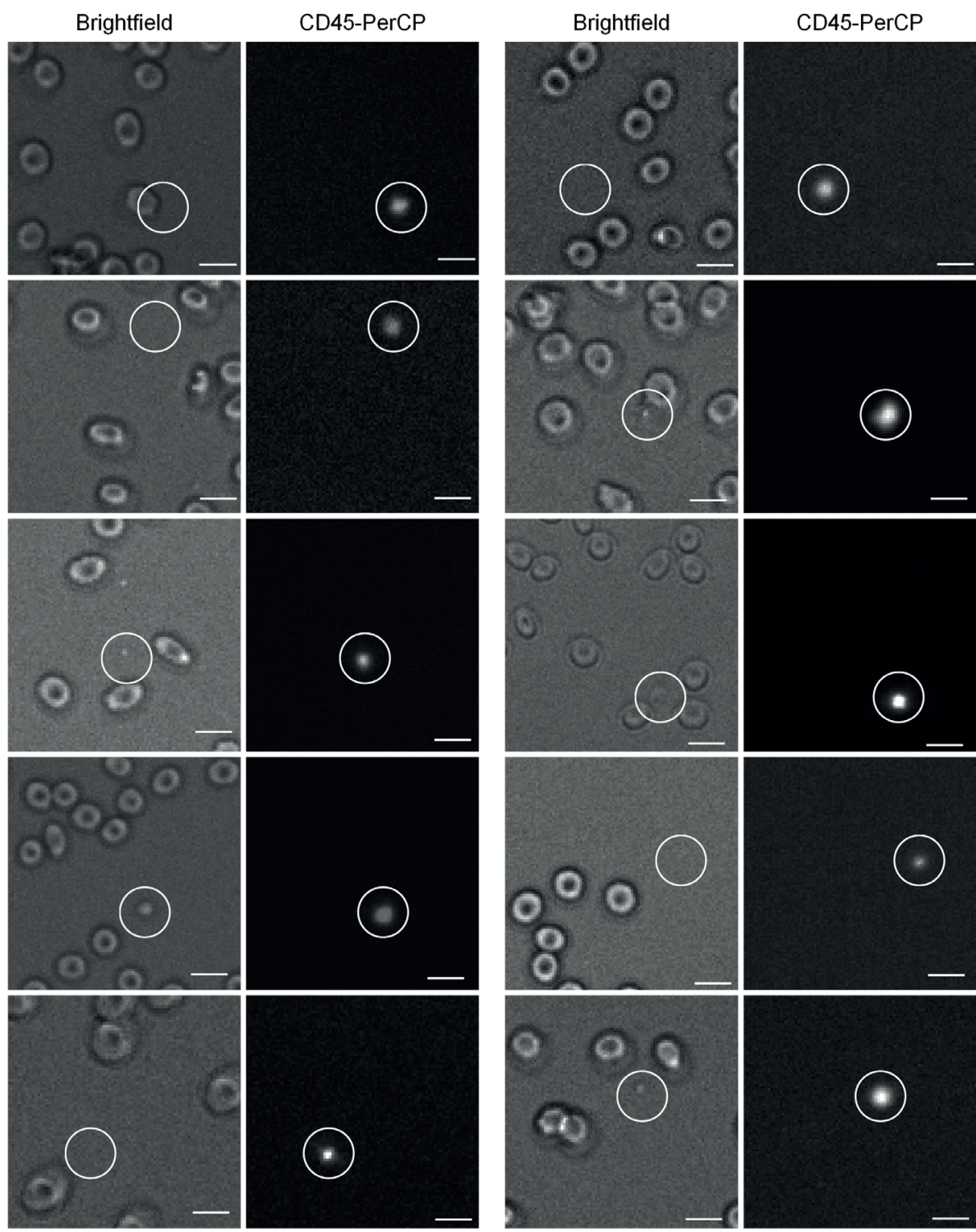

Supplementary Figure S4.2. Examples of brightfield and immunofluorescence images of leukocytederived Extracellular Vesicles, enclosed within circles. Scale bars indicate $10 \mu \mathrm{m}$. 


\title{
Chapter 5
}

\section{Scanning Electron Microscopy of Circulating Tumor Cells and tumor-derived Extracellular Vesicles}

\author{
Afroditi Nanou, Mateus Crespo, Penny Flohr, Johann S. De Bono \\ and Leon W.M.M. Terstappen
}

Cancers 2018, 10 (11), 416.

\begin{abstract}
To explore morphological features of circulating tumor cells (CTCs) and tumor-derived extracellular vesicles (tdEVs), we developed a protocol for scanning electron microscopy (SEM) of CTCs and tdEVs. CTCs and tdEVs were isolated by immunomagnetic enrichment based on their Epithelial Cell Adhesion Molecule (EpCAM) expression or by physical separation through $5 \mu \mathrm{m}$ microsieves from $7.5 \mathrm{~mL}$ of blood from CastrationResistant Prostate Cancer (CRPC) patients. Protocols were optimized using blood samples of healthy donors spiked with $\mathrm{PC}_{3}$ and LNCaP cell lines. CTCs and tdEVs were identified among the enriched cells by fluorescence microscopy. The positions of DNA+, CK+, CD45-CTCs and DNA-, CK+, CD45-tdEVs on the CellSearch cartridges and microsieves were recorded. After gradual dehydration and chemical drying, the regions of interest were imaged by SEM. CellSearch CTCs retained their morphology revealing various shapes, some of which were clearly associated with CTCs undergoing apoptosis. The ferrofluid was clearly distinguishable, shielding major portions of all isolated objects. CTCs and leukocytes on microsieves were clearly visible, but revealed physical damage attributed to the physical forces that cells exhibit while entering one or multiple pores. tdEVs could not be identified on the microsieves as they passed through the pores. Insights on the underlying mechanism of each isolation technique could be obtained. Complete detailed morphological characteristics of CTCs are, however, masked by both techniques.
\end{abstract}




\subsection{Introduction}

Circulating Tumor Cells (CTCs) play a crucial role in the formation of metastases ${ }^{1,2}$ and the CTC peripheral blood load is directly associated with the overall survival of cancer patients ${ }^{3-9}$. Many groups have focused their research on the development of different technologies ${ }^{10-13}$ to increase capture efficiency of CTCs from blood samples. Understanding the biophysical features of CTCs by scanning electron microscopy (SEM) could contribute to the future improvement of the existing or the development of new CTC isolation techniques. A detailed morphological characterization of isolated CTCs with this technology has not been studied so far. SEM has been used to visualize cells from cancer cell lines mainly on microfluidics devices or filters ${ }^{14,15}$. SEM images of CTC clusters from patient samples have also been shown previously ${ }^{16}$. However, to our knowledge, cell lines captured after being spiked into blood samples of healthy donors as well as single CTCs from the blood of cancer patients have not been studied using SEM imaging. To obtain SEM images of CTCs, we chose to isolate CTCs from the blood of Castration-Resistant Prostate Cancer (CRPC) patients by physical separation and immunomagnetic selection. For both approaches, a preparation protocol for SEM imaging needed to be developed for the optimal morphological preservation of cells once in the vacuum. Therefore, preceding the SEM imaging of CTCs, the preparation protocol was first developed and optimized on cells from tumor cell lines spiked in blood samples of healthy donors that were EpCAM enriched using the CellSearch system or isolated based on their size and deformability by the passage of blood through $5 \mu \mathrm{m}$ microsieves. The developed protocol was applied on the enriched samples from CRPC patients.

In an earlier report, we showed that CTCs have a large range of morphological appearances and proved that a large portion of them are undergoing apoptosis ${ }^{17}$. In a later study, we subdivided them into morphological subclasses, including "intact CTC", "tumor cell fragments", and "tumor microparticles", and showed that they all strongly correlated with clinical outcomes ${ }^{18}$. These EpCAM+, CK+, CD45- objects, baptized tumor-derived extracellular vesicles (tdEVs), can now be classified with objective criteria using the open-source image analysis software, Automatic CTC Classification, Enumeration and PhenoTyping (ACCEPT) (http://github.com/LeonieZ/ACCEPT), and are equivalent to CTCs in terms of prognosis of CRPC patients ${ }^{19}$. Here, we used SEM imaging to gain more insights in the morphological features of CTCs and tdEVs. 


\subsection{Materials and Methods}

\subsubsection{Cell Culture}

PC 3 and LNCaP prostate cancer cell lines were provided by the ATCC. 10,000 cells $/ \mathrm{cm}^{2}$ of $\mathrm{PC}_{3}$ and LNCaP prostate cancer cell lines were cultured in DMEM culture medium supplemented with 100 Units/mL penicillin and $100 \mu \mathrm{g} / \mathrm{mL}$ streptomycin (Lonza, cat \# 16-602E) and 10\% (v/v) FBS (Sigma-Aldrich Chemie BV, cat \# F7524, Zwijndrecht, the Netherlands). When cells reached $80 \%$ confluence, they were washed with sterile Phosphate Buffered Saline (PBS) and trypsinized using $0.05 \%$ trypsin EDTA (Gibco, cat \# 25300-062) for $2-3 \mathrm{~min}$ at $37^{\circ} \mathrm{C}$. After cell detachment, the trypsin was deactivated by an excess of FBS-containing culture medium. The cells were mixed to a homogenous cell suspension and kept in ice until further processing and spiking in blood samples.

\subsubsection{CRPC Patient and Healthy Donor Blood Samples}

$10 \mathrm{~mL}$ of Cellsave blood samples from 12 anonymous healthy donors (HDs) were obtained after written informed consent from the TNW-ECTM-donor services (University of Twente, Enschede, The Netherlands) and were used as positive controls after spiking prostate cancer cells from tumor cell lines. More specifically, 6 samples were used to spike 300-500 PC3 and 6 more to spike 300-500 LNCaP cells, and used to develop and optimize the preparation protocol after CTC isolation for SEM imaging. Half of the samples from each condition were processed by the CellSearch system (Menarini, Huntingdon Valley, PA, USA) and the other half by filtration through $5 \mu \mathrm{m}$ microsieves (VyCAP, Deventer, the Netherlands) within 2 days from the drawing day.

CellSave blood samples of 9 patients were provided by the Royal Marsden Hospital and processed within 2-3 days from the drawing day with either the CellSearch system (5 samples) or filtered through $5 \mu \mathrm{m}$ pore microsieves (4 samples). The trial CCR2472 was approved by the Royal Marsden Research Ethics Committee (ethics reference number: 04/ Q0801/60). Patients had metastatic prostate cancer progressing despite castrate levels of testosterone after histological confirmation and had provided written informed consent to trial protocols approved by the institutional review boards at each participating center.

\subsubsection{Immunomagnetic CTC and tdEV Isolation}

$7.5 \mathrm{~mL}$ of blood samples were centrifuged at $800 \times \mathrm{g}$ for $10 \mathrm{~min}$ without breaks. The plasma ( $\mathrm{x}$ volume) $1 \mathrm{~cm}$ above the buffy coat was removed and $(5.5+\mathrm{x}) \mathrm{mL}$ of dilution buffer was added to the samples, mixed gently, and centrifuged again at $800 \times g$ for 10 min without breaks. The EpCAM+ CTCs and EpCAM+tdEVs were positively selected by immunomagnetic beads (ferrofluid) conjugated with $\alpha$ EpCAM from $7.5 \mathrm{~mL}$ of blood of cancer patients using the CTC kit in the CellSearch system as previously 
described $^{20}$. Briefly, the centrifuged samples were added in the fully automated CellTracks Autoprep. Once the interface between the red blood cells and the remaining diluted plasma was detected, the diluted plasma was discarded. aEpCAM ferrofluid of the CTC kit together with a capture enhancement reagent were added in the samples. Cells within the tube were surrounded by 3 magnets moving backwards and forwards to increase the collisions between the ferrofluid and the cells. When the bound to ferrofluid cells were accumulated at the bottom of the tube, ferrofluid-free cells were discarded. The enriched objects (CTCs, leukocytes, and EVs) were permeabilized with saponin and incubated with the staining solution of the CTC kit containing the nuclear dye DAPI, antibodies against epithelial-specific cytokeratins $\alpha$ CK-PE (clones C11 and $\mathrm{A} 53-\mathrm{B} / \mathrm{A} 2$ ), and an antibody against the leukocyte-specific cluster of differentiation $\mathrm{CD} 45, \alpha \mathrm{CD} 45-\mathrm{APC}$. Following some incubation and washing steps, cells were fixed and loaded into a cartridge placed within a magnest for their imaging using a semiautomatic 10x objective fluorescence microscope, the CellTracks Analyzer II. The Cellsearch definition of a CTC was to have a cell-like morphology, have a nucleus (DAPI+), express cytokeratin (CK+), have a size bigger than $4 \mu \mathrm{m}$, and do not express CD45 (CD45-). tdEVs were defined as particles expressing only cytokeratin (DNA-, $\left.\mathrm{CK}+, \mathrm{CD}_{45}-\right)$ as previously described ${ }^{19}$.

\subsubsection{Size-Based CTC Isolation}

7.5-10 mL of CellSave blood samples of CRPC patients and healthy donors spiked with prostate cancer cell lines were filtered through $5 \mu \mathrm{m}$ microsieves provided by VyCAP. 100 mbar was applied to the filters using a pump unit. Once the whole volume of blood was filtered, cells were washed once with $0.15 \%(w / v)$ saponin in $1 \%(w / v)$ BSA in PBS. Permeabilization using $0.15 \%(w / v)$ saponin in $1 \%(w / v)$ BSA in PBS for 15 min at room temperature (RT) was followed. A staining solution containing $1 \mu \mathrm{g} / \mathrm{mL} \alpha \mathrm{CKs}$ (clone C11) -PE (Veridex, cat \#PN6030), $2 \mu \mathrm{g} / \mathrm{mL} \alpha$-Pan CK-efluor 570 (eBioscience, cat \#41-

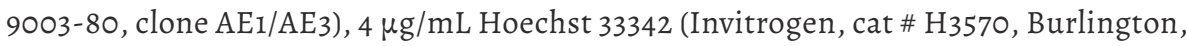
Canada), and $4 \mu \mathrm{L}$ of $\alpha \mathrm{CD} 45$-PerCP (Invitrogen, cat \#MHCD4531) in a final volume of $50 \mu \mathrm{L}$ of $0.05 \%(w / v)$ saponin in $1 \%$ BSA in PBS was applied in each sample for $15 \mathrm{~min}$ at $37^{\circ} \mathrm{C}$ in a humidified environment. Two washing steps were followed. The cells were finally fixed with $1 \%(v / v)$ formaldehyde in PBS for $10 \mathrm{~min}$ at RT. One more washing step using $1 \%(w / v)$ BSA in PBS was used. The cells on the microsieves were further prepared as described in section 5.2 .5 before being imaged using a $20 \times$ objective fluorescence microscope.

\subsubsection{Specimen Preparation for Scanning Electron Microscopy (SEM)}

Isolated objects in the CellSearch cartridges or on the microsieves were fixed with $4 \%$ formaldehyde for $15 \mathrm{~min}$ at RT to better retain their morphology in the following preparation steps. Subsequent dehydration was required to exchange the high watercontent of cells and EVs with ethanol (that has lower surface tension to air than water to air) and hinder cell deformation and corruption when in the vacuum chamber of 
the SEM. Therefore, gradual dehydration from $70 \%(v / v)$ ethanol in milliQ up to $100 \%$ ethanol with a $10 \%(v / v)$ ethanol concentration increment step $(70 \%, 80 \%, 90 \%, 100 \%$ $(v / v)$ ethanol) took place at RT. Each dehydration step lasted for 5-10 min at RT. After $100 \%$ ethanol, 1:1 ethanol:hexamethyldisilazane (HMDS) was added to the cells for 3-5 min at RT followed by a final step of 3-5 min incubation in HMDS and air drying.

In the case of CellSearch cartridges, the glass slides were removed from the cartridges. All specimens were left overnight at RT for the HDMS residual to be evaporated.

Since SEM is based on the electrons passing through the material of interest, an electrically conductive sample is required. Cells and tdEVs as well as glass slide and microsieve substrates have a low electron density, resulting in reduced electron scattering and low quality images, sample charging, damage and carbon deposition; hence, a metal coating is required. Herein, $10 \mathrm{~nm}$ gold coating was applied using the JEOL JFC-1300 Auto Fine Coater (10 times of $1 \mathrm{~nm}$ thickness gold was applied using $10 \mathrm{~mA}$ for $30 \mathrm{~s}$ ).

\subsubsection{Scanning Electron Microscopy (SEM)}

The dehydrated and dried specimens were mounted on silicon holders with a both sided glue carbon tab and placed in the vacuum chamber of SEM (JEOL JSM-6610LV) for further analysis. A region of interest (ROI) containing single or multiple cells was first localized with SEM using relatively low magnification ( 350x). The SEM was operated at $4-5 \mathrm{kV}$ acceleration voltage using secondary electron (SE) detection. Once the regions of interest were relocated following the cell patterns of the respective fluorescence images, SEM images of CTCs and tdEVs were recorded using a higher magnification $(3000 \times$ to $10,000 \times)$.

\subsection{Results}

\subsubsection{Cell Preparation for SEM Imaging}

A preparation protocol of cells for their subsequent SEM imaging was developed as described in section 5.2.5. PC3 and LNCaP cells in suspension were used for the optimization of the protocol. A diagnostic leukapheresis (DLA) sample of a prostate cancer patient sample was used to obtain SEM images of the leukocytes (Figure 5.1, Panels A and D). The protocol preserved cell shape, morphology, and distinct surface features distinguishing leukocytes, PC3, and LNCaP cells (Figure 5.2, Panel A). The sizes of PC3 and LNCaP cells were much larger as compared to the sizes of leukocytes.

\subsubsection{Overview of Cells by SEM Imaging on Glass Slides, CellSearch Cartridges, and $5 \mu \mathrm{m}$ Pore Microsieves}

Figure 5.1 shows the SEM images of leukocytes on a glass slide (Panels A and D), after immunomagnetic enrichment on a CellSearch cartridge (Panels B and E) and on 
microsieves with $5 \mu \mathrm{m}$ pores (Panels $\mathrm{C}$ and F). Panels A-C are shown in lower (330350x) magnification and panels $\mathrm{D}-\mathrm{F}$ at a higher (3300-3500x) magnification. Cells were found mainly in aggregates when prepared in cell suspension for SEM imaging; however, their surface features were very clear. On the other hand, the isolated cells on the CellSearch cartridge were in a monolayer and fully covered by the ferrofluid that is aligned according to the magnetic field lines ${ }^{21}$. Many cells on the microsieves were damaged. Some cells kept their spherical shape, without, however, keeping their cell surface features, probably due to the stress they experienced while entering the pores.
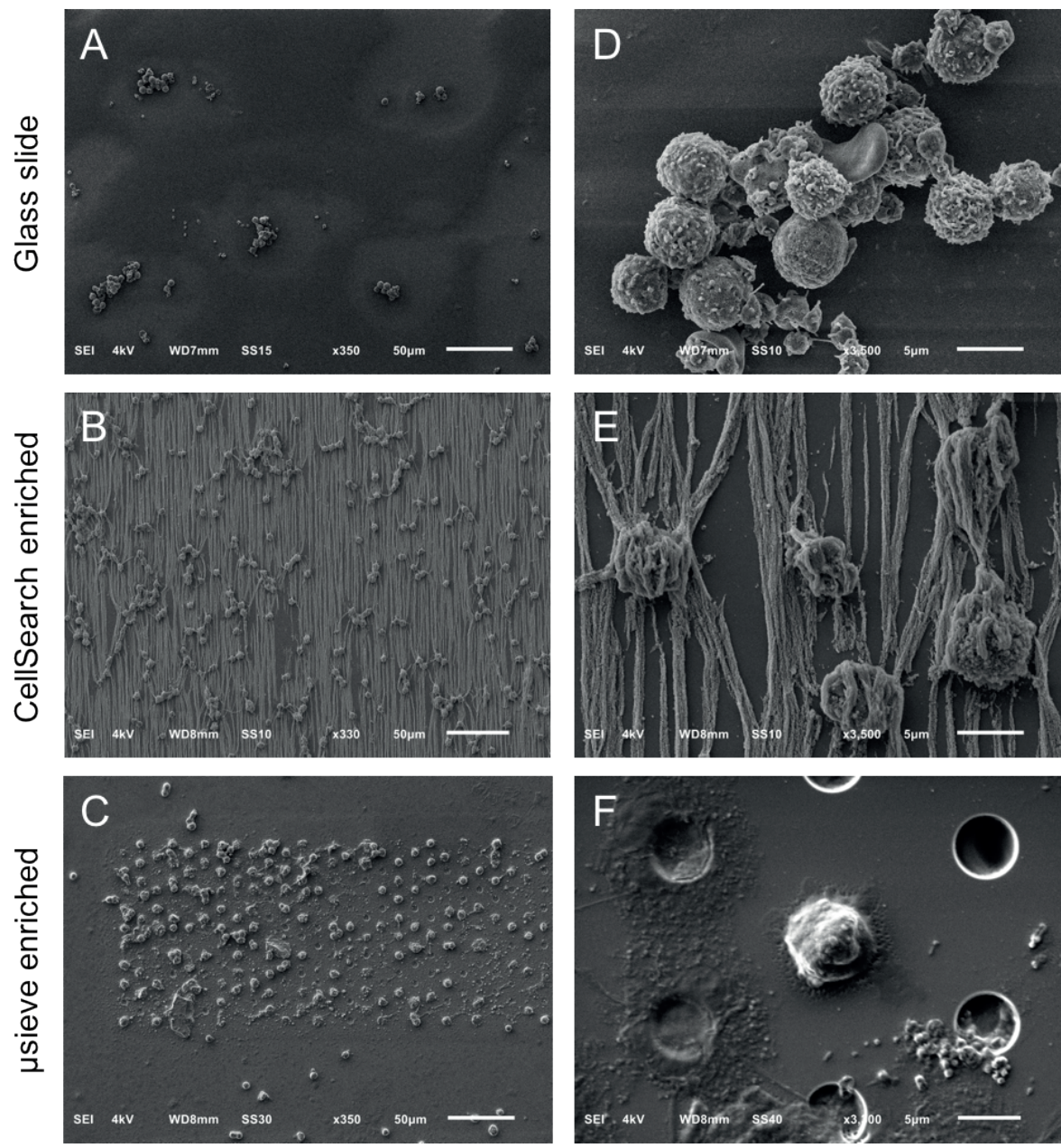

Figure 5.1. Scanning Electron Microscopy Images of cells in suspension and after size- and EPCAMbased enrichment. Overview of cells by SEM on a glass slide (Panel A), a CellSearch cartridge (Panel B) and a $5 \mu \mathrm{m}$ pore microsieve (Panel C). A higher magnification of the isolated cells is shown at the right of each technique (Panels $D-F$ ). The vertical lines in panels $B$ and $E$ is the $\alpha$ EpCAM ferrofluid covering the surfaces of all isolated objects and are perfectly aligned with the magnetic field lines ${ }^{21}$. 


\subsubsection{SEM Imaging of Isolated PC 3 and LNCaP Cells Spiked in Blood by the CellSearch System and Microsieves}

SEM images of $\mathrm{PC}_{3}$ and $\mathrm{LNCaP}$ cells in suspension were compared to the $\mathrm{PC}_{3}$ and LNCaP cells, which were isolated by CellSearch or on microsieves after being spiked in blood samples (Figure 5.2). In most of the cases, the PC 3 and LNCaP cells that were isolated by CellSearch were fully covered by ferrofluid. The images shown in panel B of Figure 5.2 reveal small parts of their surfaces. In these minor exposed parts, PC3 and LNCaP cells seem to have retained their distinctive morphological features, with $\mathrm{PC}_{3}$ having more heterogeneous surface with very smooth portions and elongated microvilli in some parts whereas LNCaP was more homogeneous with microvilli covering all their surface. Nonetheless, in the case of microsieves, apart from few LNCaP cells that seemed to have some well-preserved their surface characteristics, most of the isolated cells lost their distinctive microvilli that were found when cells were in suspension. Moreover, many DNA+ cells after being relocated and SEM imaged, were flattened with surface defects, implying that their nucleus was inside the pores and only the membrane was left on the top. Other cells were within more than one pore, indicating that cells underwent a lot of stress during their filtration. Stripes connecting the neighboring pores could also be observed (Figure 5.2, Panel C, PC 3 cells), implying the fragmentation of formerly passed cells through the $5 \mu \mathrm{m}$ pore microsieves or the cell tearing of the imaged ones.

\subsubsection{Relocation and Correlated SEM-Fluorescence Images of CTCs and tdEVs of CRPC Patients Isolated by the Cellsearch}

After immunomagnetic enrichment by Cellsearch, all objects are covered by ferrofluids, shielding a major portion of the cell surfaces. Hence, to ensure which of the enriched cells were CTCs, the region of interest containing the CTCs from the fluorescence images were found using SEM. Figure 5.3 shows an example: In Panel A, one of the fluorescence images of a cartridge of a CRPC patient is shown, with nucleus (DNA) shown in blue, CD45 (membrane marker of leukocyte) shown in red, and cytokeratin CK (intracellular marker of epithelial cell) shown in green. The corresponding SEM image is shown in Panel B, and the position of individual cells in both fluorescent and SEM images is indicated with numbers. Sometimes, cells (\#14 is an example) could not be relocated in the SEM image and were most likely removed through the dehydration/ drying procedure. The yellow square in Panels A and B encloses two cells, one of which is clearly a CTC and the other one is of unknown lineage of origin as no CD45 or CK staining could be discerned. Both cells are shown at higher magnification in Panels $\mathrm{C}$ and $\mathrm{D}$. The cytokeratin of the CTC shows a punctuated pattern that is characteristic for CTC undergoing apoptosis ${ }^{17,22}$. This punctuated pattern cannot be seen in the SEM image either because the CTC is covered by the ferrofluid or because this pattern is only intra-cytoplasmatic. The lineage of origin of the other cell is not known, the nucleus does not show the typical shape of a granulocyte that can be seen in other nuclei and there is no staining with CD45. Big vesicles at the surface, as indicated 
with the arrows in Panel D (Figure 5.3), were seen in 55\% of the imaged leukocytes (47 out of 85 ) as shown in Panel A, Figure S5.5.15\% of leukocytes (13 out of 85 ) had smaller vesicles on their surfaces (Panel B, Figure S5.5) similar to the ones observed on $52 \%$ of the imaged CTCs (11 out of 21) we identified (Figures 5.4 and S5.1). Hence, the finding of vesicles solely on the surface of an isolated cell without CK (or any other epithelial/ tumor-specific) staining is not sufficient to suggest that this is indeed a CTC.

A. On a glass slide
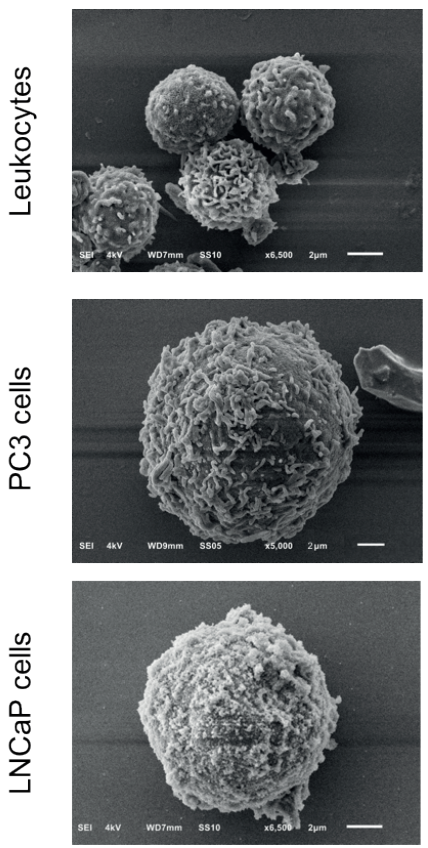

B. CellSearch enriched
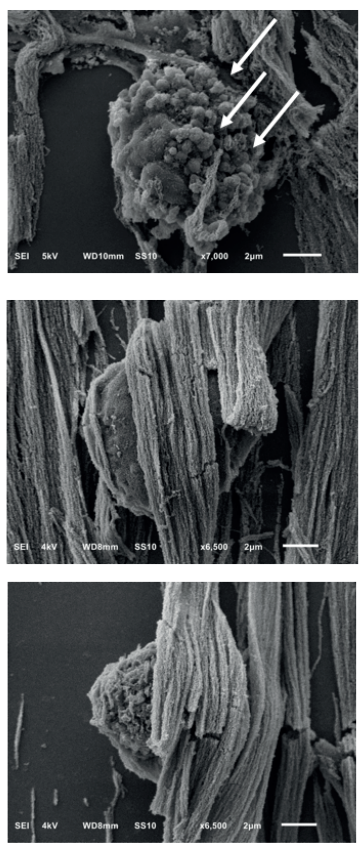

C. $\mu$ sieve enriched
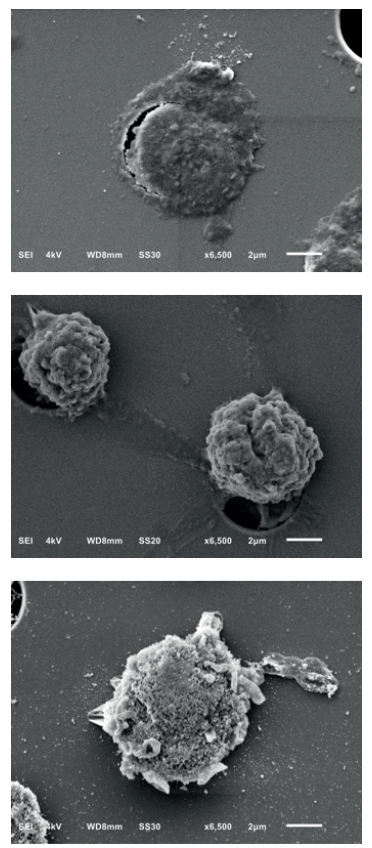

Figure 5.2. SEM images of leukocytes, $\mathrm{PC}_{3}$, and $\mathrm{LNCaP}$ cells on a glass slide (Panel A) and after being spiked in blood and isolated by the Cellsearch (Panel B) and on $5 \mu \mathrm{m}$ pore microsieves (Panel C). The arrows (Panel B) are pointing at spherical vesicles on the surface of a leukocyte isolated by the CellSearch.

Following the same procedure, CTCs of CRPC patients identified by the CellSearch system were relocated using SEM and a gallery of correlated fluorescence and SEM images of CTCs was constructed (Figures 5.4 and S5.1).

Most of the CTCs have a comparable size to the leukocytes isolated by the CellSearch system as shown in Figure 5.3, supporting the previous findings of Ligthart et al. that $20-40 \%$ of CTCs in breast, prostate, and colorectal cancer patients have a diameter close to $10 \mu \mathrm{m}$ similar to the diameter of leukocytes ${ }^{23}$. On the contrary, PC 3 and LNCaP cells that were used as controls for the optimization of the protocols were clearly 
bigger than leukocytes and easily distinguishable from the rest of the leukocytes even when low magnification was used with SEM.
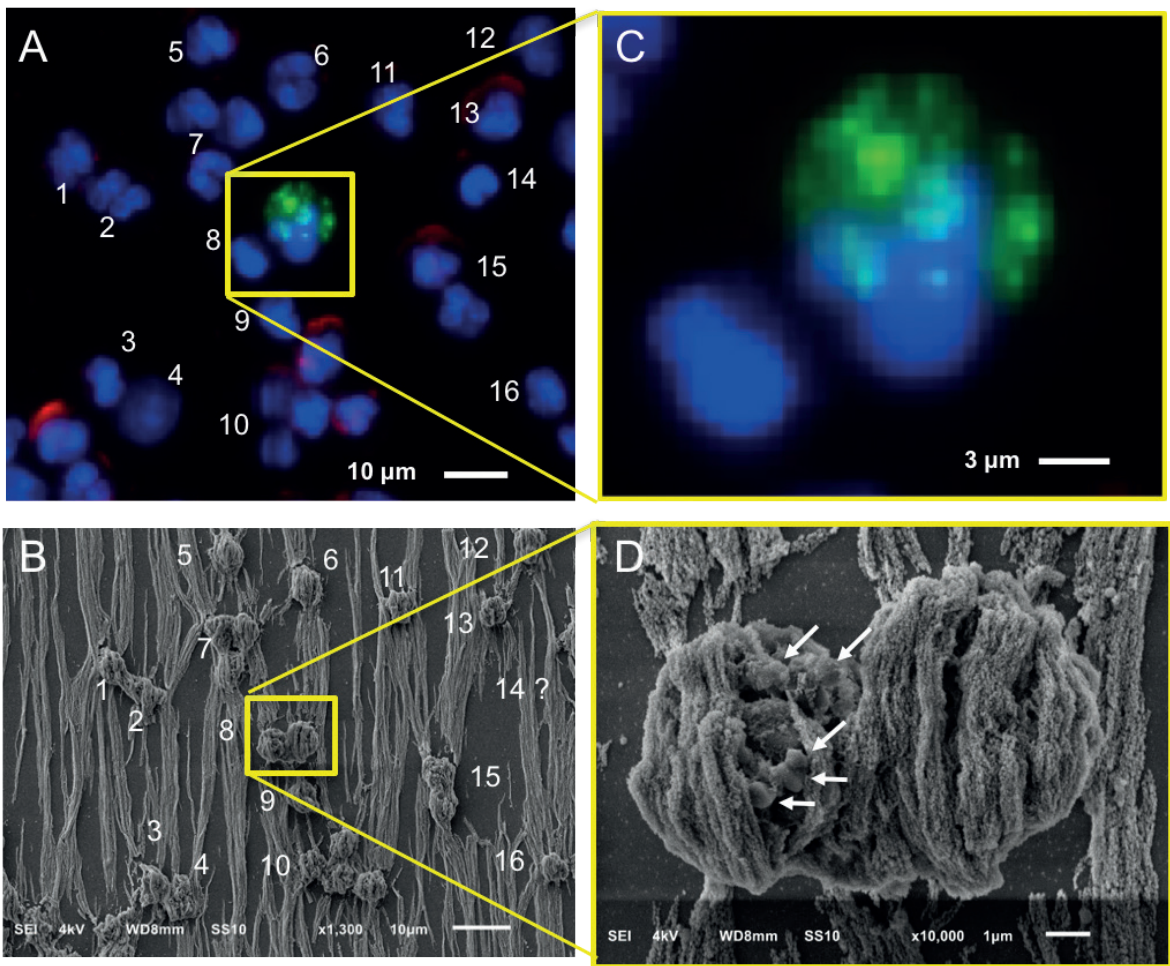

Figure 5.3. Relocation of an identified CTC of a CRPC patient by fluorescence (CellTracks Analyzer II) imaging (Panels $A$ and $C$ ) after immunomagnetic enrichment using the CellSearch system and SEM imaging (Panels B and D). Magnified fluorescence and SEM images of the enclosed by the yellow square CD45-, CK- nucleated cell (left), and CD45-, CK+ apoptotic CTC (right) are shown at Panels B and D, respectively. Nucleus (DNA) is represented by blue, CK by green, and CD45 by red. The arrows of Panel D are pointing at vesicles on the surface of a cell of unknown lineage (DNA+, CK-, CD45-).

Different shapes of CTCs were found in the CellSearch cartridges as shown in Figures 5.4 and S5.1. 67\% of CTCs (14 out of 21) were spheroid to ovoid (Panels C, D of Figure 5.3, Panels A,E-F,H of Figure 5.4, Panels A-C,E-F,H,J-L of Figure S5.1). Interestingly, $19 \%$ of CTCs (4 out of 21) bore a protrusion from one side (Panels B, C of Figure 5.4, Panel D of Figure S5.1) or from both sides, resembling a sigmoidal shape (Panel I, Figure S5.1), which was not expected from the respective fluorescence image. 19\% of CTCs (4 out of 21) were eight-shaped (Panels C, D, G of Figure 5.4, Panel G of Figure S5.1) likely corresponding to different phases of a CTC cycle, such as telophase (Panel D of Figure 5.4) and anaphase (Panel G of Figure S5.1). On the contrary, PC3 and LNCaP cells (Panel A, Figure S5.4) appeared larger and more homogeneous with a spheroid to ovoid shape. 

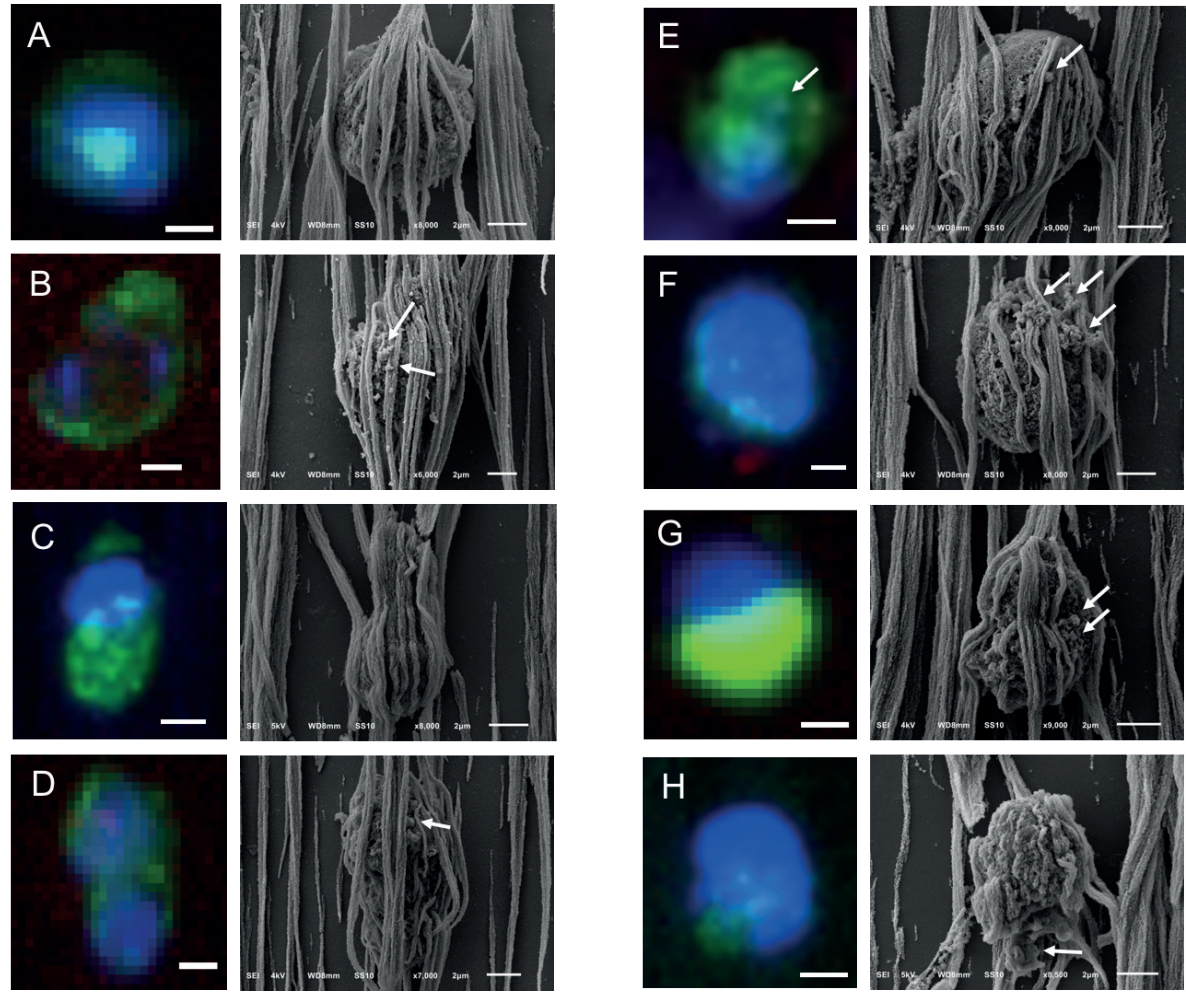

Figure 5.4. Gallery of correlated fluorescence and SEM images of CTCs of a CRPC patient isolated by the Cellsearch system. Nucleus (DNA) is represented by blue and CK by green. The arrows are pointing at spherical vesicles on the surface of CTCs (Panels D-G), single or aggregated vesicles on top of the ferrofluid covering the CTCs (Panels B and F), and bigger CK+ particles or membrane fragments of a not well defined shape (Panel H). Scale bars on fluorescence images indicate $3 \mu \mathrm{m}$.

The surface of CTCs appeared to be smooth and sponge-like because of some holes they seem to have on their surface regardless of their speckled cytokeratin, as denoted by arrows on Panels G,I,J of Figure S5.1. In addition, the 21 CTCs that were imaged from two different patients did not have distinctive microvilli as PC 3 and LNCaP cells (Panels A and B of Figure 5.2, Panel A of Figure S5.4); it is expected that once tumor cells intravasate and circulate in the bloodstream, they undergo epithelial to mesenchymal transition (EMT), obtaining a more mesenchymal phenotype. During that process, it is expected that they will not need specific receptors to grow as while adherent.

In many cases, CTCs had vesicles, as indicated by arrows in Figures 5.3, 5.4 and S5.1. The CTC of Panel E, Figure 5.4 has a vesicle on its surface that corresponds to a cytokeratin speckle in the respective immunofluorescence image, implying that the secreted vesicles of CTCs express also cytokeratin. The ferrofluid covering all the cells, 
however, hinder their morphological characterization in depth.

In Panels B and C of Figure S5.1, the speckled cytokeratin pattern of the apoptotic CTCs corresponds to extra-cytoplasmatic vesicles. Some of these vesicles are clearly above the ferrofluid, raising questions about their origin. Are they vesicles extruded from the CTCs' surface during their capture by the $\alpha$ EpCAM ferrofluid or vesicles not related to the specific CTCs that were present in the enriched cell suspension? The hypothesis that they are vesicles derived from the CTC can be supported by the observation that similar structures are seen on some CTCs found on the microsieves (Figure S5.2, Panels D,H,J,N,O,S,T). On the other hand, the fact that these particles were not only found in the close vicinity of the specific CTCs, but also spread in a part of the cartridge also covering leukocytes, would support the second hypothesis. However, both cases could be true.

\subsubsection{Relocation and Correlated SEM-Fluorescence Images of tdEVs of CRPC Patients Isolated by the Cellsearch}

The same procedure that was described for the SEM imaging of the CTCs was followed in the case of EpCAM+tdEVs isolated from the blood of CRPC patients by Cellsearch. An example is shown in Figure 5.5. A DNA-, CK+, CD45-tdEV is surrounded by leukocytes (Panel A). The same tdEV can be hardly distinguished at 1000x magnification in the respective SEM image (Panel B) in contrast to the easily recognizable cells. The small size of tdEVs in combination with the ferrofluid covering them as well as the rest of the surface of the cartridge hinders their $3 \mathrm{D}$ shape and makes their relocation laborious. Due to that, a limited number of tdEVs (only six) were SEM imaged. However, as we have demonstrated previously, tdEVs can be found in significantly elevated numbers in CRPC patients (median value of 116 tdEVs in 84 CRPC patients) compared to healthy donors (median value of $8 \mathrm{tdEVs}$ in 16 healthy donors) ${ }^{19}$. The correlated fluorescence and SEM images of tdEVs of CRPC patient blood samples are summarized in Figure 5.6. Isolated tdEVs are much smaller compared to leukocytes, nucleated cells, or CTCs, with the majority of them having a size range of $1-2 \mu \mathrm{m}$ based on the SEM images of Figure 5.6. The shape of all tdEVs shown here is spheroid to ovoid; however, digitally stored fluorescence images of CRPC patients imply also a subpopulation of tdEVs with a tubular shape. However, this shape is less frequent $(5-10 \%$ of the total tdEV population, data not shown). Different $\mathrm{CK}$ intensities can be recorded in the fluorescence images of tdEVs, with some of them having very dim CK signals (Panels E and F). Nevertheless, the SEM images confirm that they are not just image artefacts, but actual particles isolated by the blood samples of the patients in the same manner as CTCs as shown by the ferrofluid covering them. The parts of tdEVs that are shielded by the ferrofluid have lower CK signals, denoting that the ferrofluid is reducing the resulting fluorophore signals, which can be crucial in the case of small EVs to be detected by solely one biomarker. 

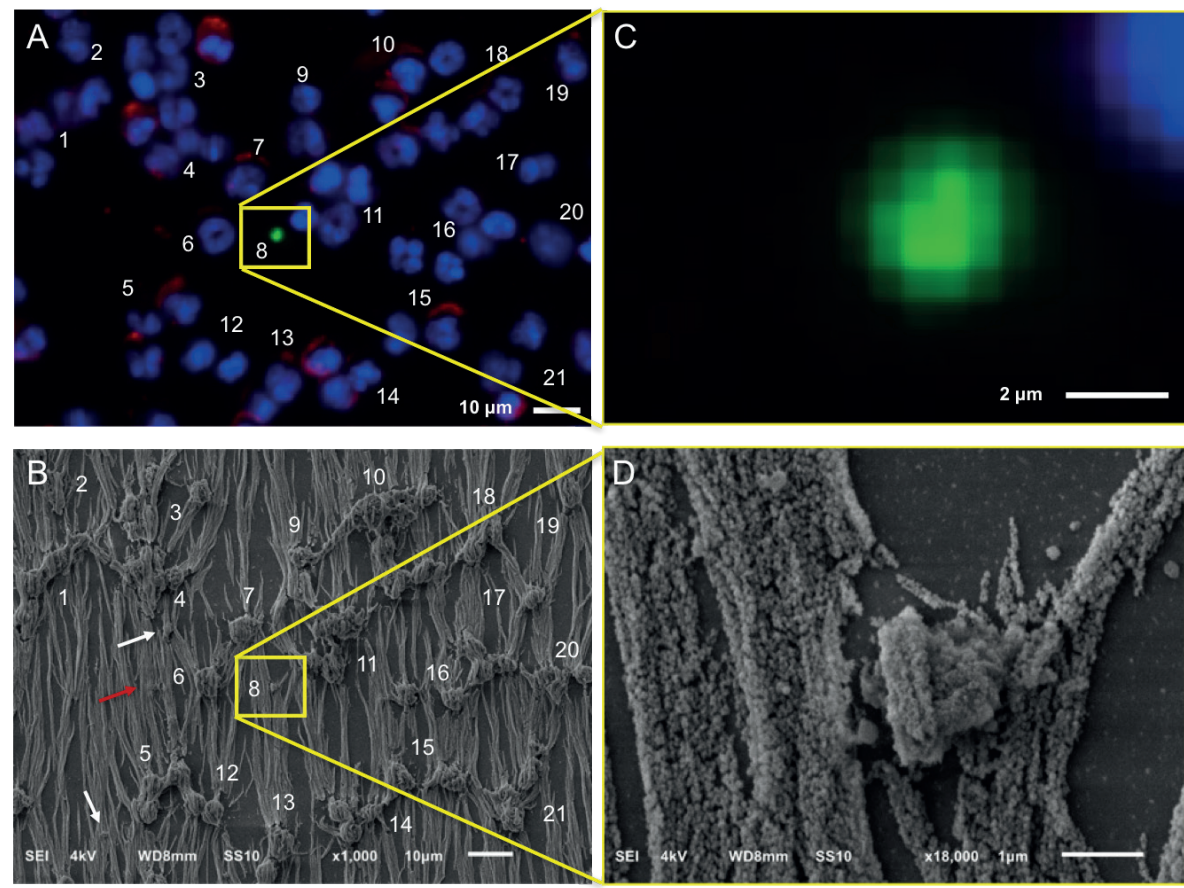

Figure 5.5. Relocation of an identified tdEV of a CRPC patient by fluorescence (CellTracks Analyzer II) imaging (Panels A and C) after immunomagnetic enrichment using the CellSearch system and SEM imaging (Panels B and D). Magnified fluorescence and SEM images of the enclosed by the yellow square DNA-, CK+, CD45- tdEV are shown at Panels B and D, respectively. Nucleus (DNA) is represented by blue, CK by green, and $\mathrm{CD} 45$ by red. The white arrows (Panel B) are pointing at vesicles of not defined origin (DNA-, CK$\mathrm{CD} 45-$ ). The red arrow (Panel B) is pointing at a leukocyte derived- extracellular vesicle (DNA-, $\left.\mathrm{CK}-, \mathrm{CD} 45^{+}\right)$.

A DNA-, CD45+ particle is indicated by a red arrow in Figure 5.5 (Panel B), suggesting that particles of leukocyte origin are co-isolated with the CellSearch system. Further investigation is required to answer the raised question of whether these particles are fragments as a result of the CellSearch procedure or actual extracellular vesicles (EVs) secreted by leukocytes.

It is noteworthy to mention that more particles are suspected to be isolated by the CellSearch system that cannot be detected by fluorescence because either they do not express any CD45 or CK, or they express CD45 or CK at levels below the detection limits of our fluorescence microscopes. Some of these particles that have a very similar shape and morphology as the tdEV of Figure 5.5 are pointed out by white arrows in Figure 5.5 (Panel B); however, the highlighted particles were not SEM imaged with a higher magnification. A higher magnification of a different particle undetectable by the CellTracks Analyzer II (DNA-, CD45-, CK-) was SEM imaged and can be found in Figure S5.3. 

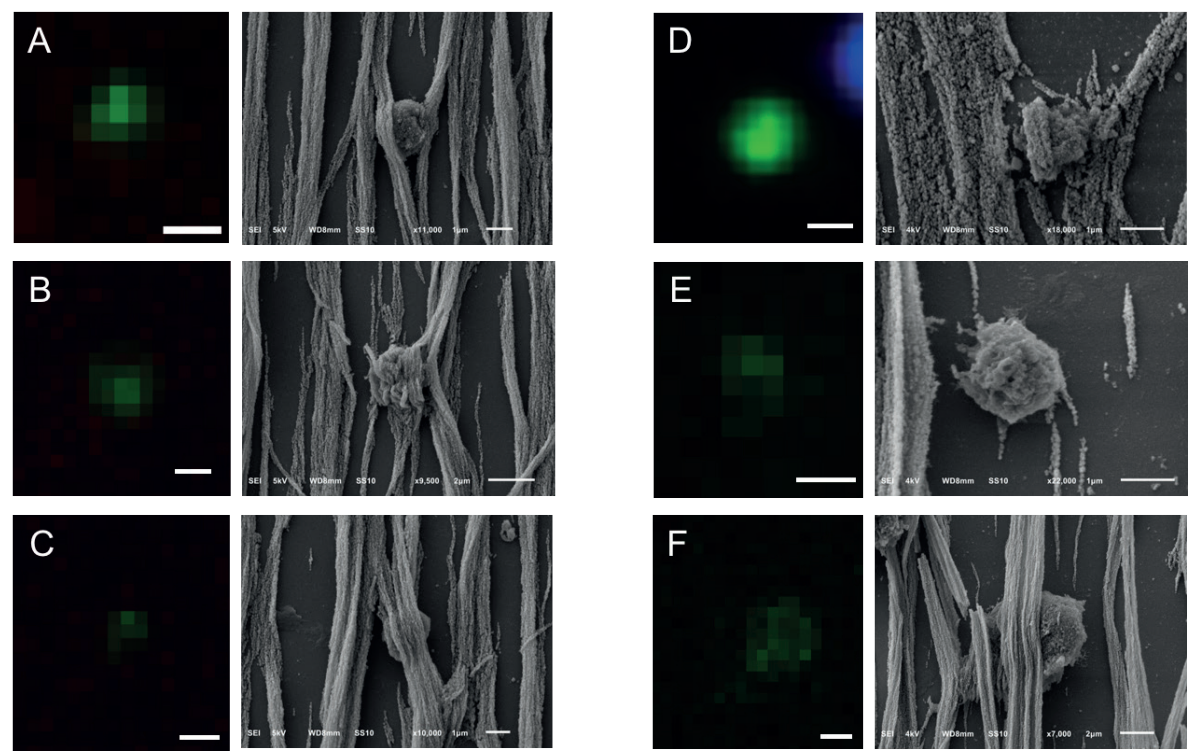

Figure 5.6. Gallery of correlated fluorescence and SEM images of tdEVs of a CRPC patient isolated by the Cellsearch system. CK is represented by green. The pixels with lower CK intensity (Panels $A-C, F)$ correspond to being shielded from ferrofluid tdEV parts. tdEVs of different $C K$ intensities can be observed (Panel D: High CK intensity, Panels A-C: Medium CK intensity, Panels E,F: Low CK intensity). Scale bars of fluorescence images indicate $2 \mu \mathrm{m}$.

\subsubsection{Relocation and Correlated SEM-Fluorescence Images of CTCs of CRPC Patients Isolated by Microsieves}

The recommended VyCAP protocol was first applied for the permeabilization, fixation, and staining of cells isolated by microsieves. However, that led to a lot of red blood cell background during their SEM imaging, most likely due to the fixation step that preceded the cell permeabilization step (Figure S5.6); hence, a different protocol that is similar to the protocol used by the Cellsearch was eventually followed for both control and patient samples and is described in Section 5.2.4.

Most spiked PC 3 and LNCaP cells in blood, after being filtered through the microsieves, had lost their surface features (Figures 5.2 and S5.4). The loss of surface features was tested in 20-30 different spiked cells ( $\mathrm{PC}_{3}$ or $\left.\mathrm{LNCaP}\right)$ in three replicates. Therefore, the relocation of identified DNA+, $\mathrm{CK}+, \mathrm{CD} 45-\mathrm{CTCs}$ based on the fluorescence images had to be relocated using SEM (Figure 5.7). The procedure was less time consuming compared to SEM imaging of the CTCs in the CellSearch cartridges thanks to the specific pore locations of the microsieves. Cells in Figure 5.7 with bright DNA signals $(\# 2,3,7,9-11)$ seemed to be more intact compared to the cells with dim DNA signals $(\# 1,4,6)$, which appeared to be damaged or within the pores. 
By relocating the CTCs on microsieves from the whole blood of one CRPC patient, we constructed a gallery of correlated fluorescence and SEM images of 28 CTCs shown in Figures 5.8 and S5.2. CTCs were found on top, within, or between the pores of the microsieves.
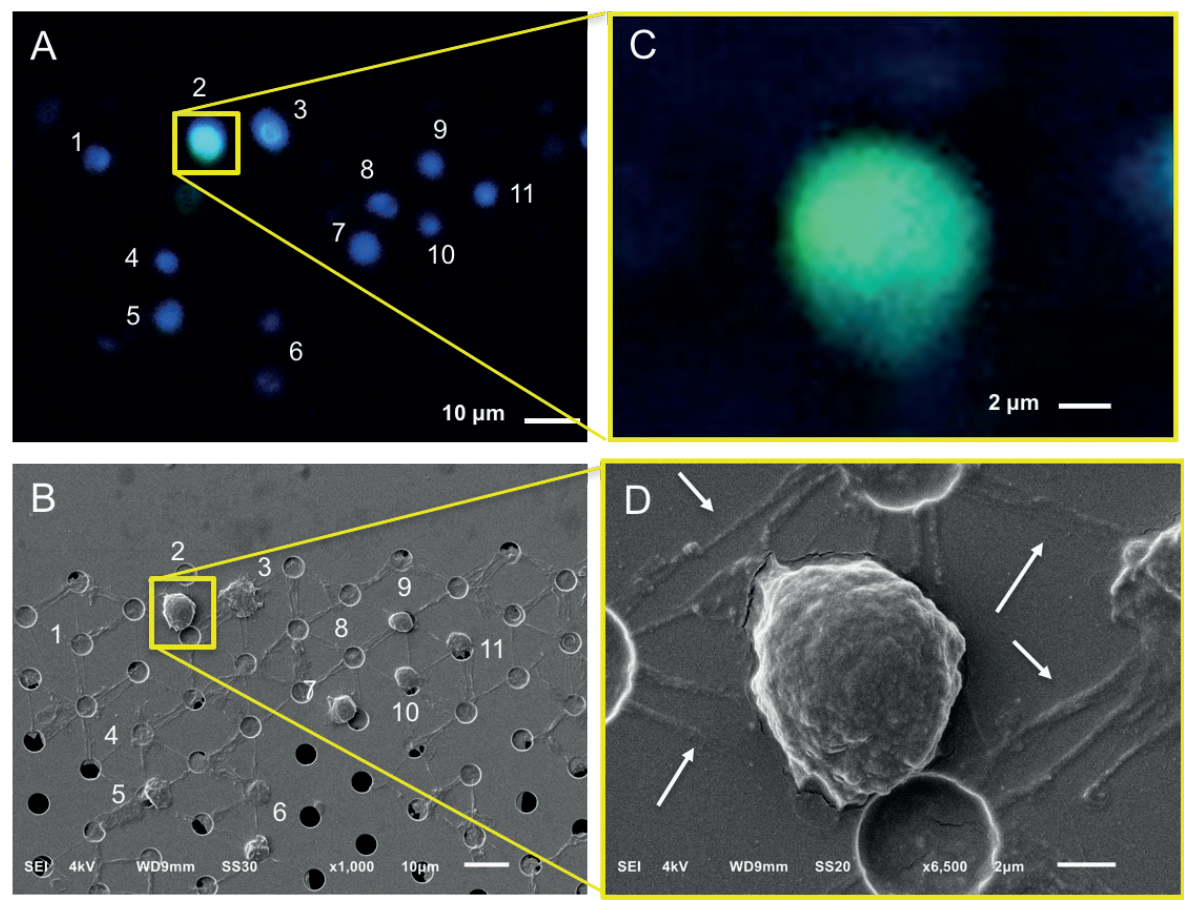

Figure 5.7. Relocation of an identified CTC in a blood sample of a castration-resistant prostate cancer patient after microsieve filtration and fluorescence imaging (Panels A and C). Pores were relocated and the respective cells were SEM imaged (Panels B and D). Nucleus (DNA) is represented by blue and CK by green. Fluorescence and SEM images of the enclosed by the yellow square DNA+, CK+, CD45- CTC at Panels A and B are shown with higher magnification at Panels C and D, respectively. Arrows (Panel D) are pointing at the membrane or/and nucleus stripes of cells connecting the neighboring pores.

The SEM images showed some stripes connecting the neighboring pores (indicated by arrows on Panel D of Figure 5.7), implying that the cells were split into more than one pore during their passage through the microsieves. These stripes were either cell membranes or nuclei as indicated by the respective immunofluorescence images of CTCs (Figures 5.7, 5.8 and S5.2). More specifically, the CK staining of Panel C of Figure 5.8 corresponds to the membrane of a CTC reaching the pore, whereas the DNA signal of panel B of Figure 5.8 and Panel C of Figure 5.7 corresponds to the fragmented nuclei of previously passed cells through the microsieves. In some cases, it is not clear whether these stripes originate from the cells occupying a pore or from previous cells that passed during blood filtering (Panels A,H of Figure 5.8). 

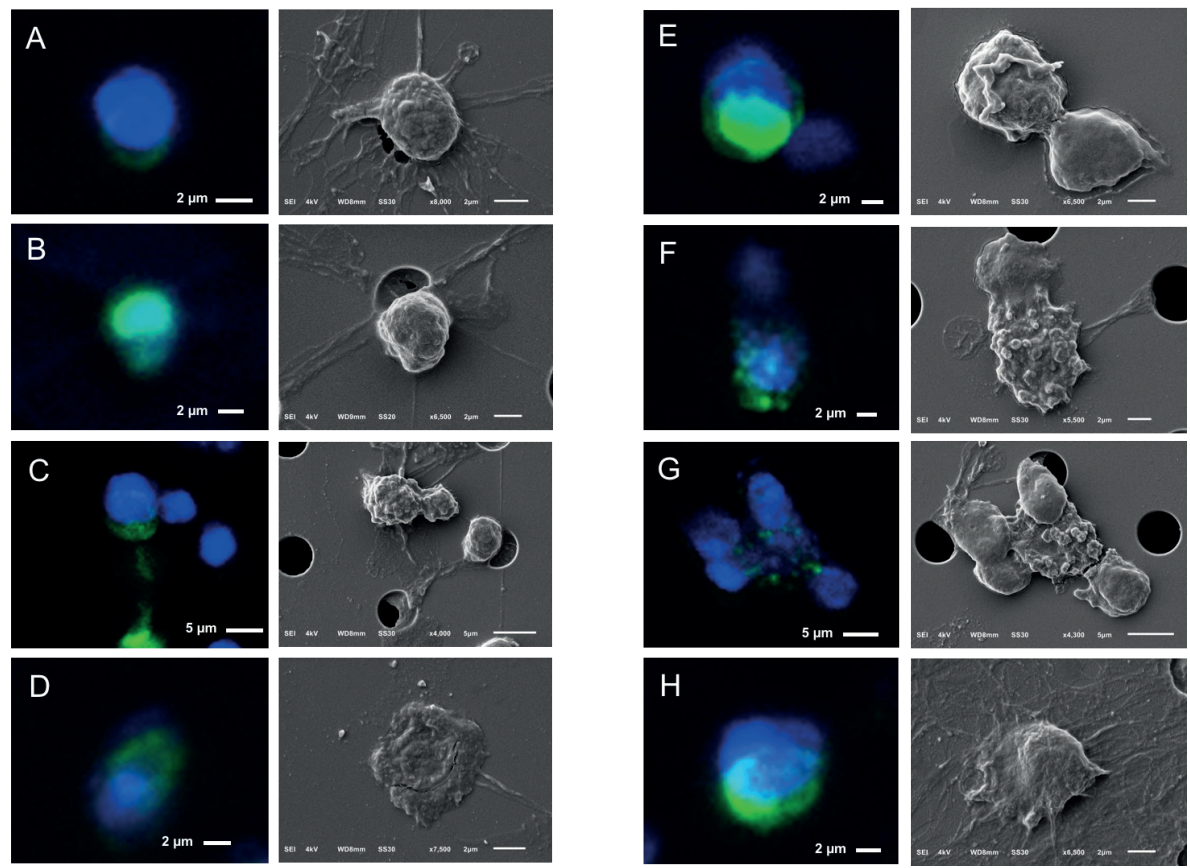

Figure 5.8.Gallery of correlated fluorescence and SEM images of CTCs of a CRPC patient isolated by whole blood filtration using $5 \mu \mathrm{m}$ microsieves. Nucleus (DNA) is represented by blue and CK by green. Membrane (Panel C) or nucleus (Panel B) stripes connecting the neighboring pores indicate cell damage and were found throughout the whole microsieve. CTCs ended up on (Panels A-C, F, H), within (Panel D), or in between the pores (Panels E, G, H).

Besides "intact" CTCs, apoptotic CTCs with a punctuated cytokeratin pattern were found on the microsieves (Panels F,G of Figure 5.8). The vesicles on their cell surface suggest that the CK punctuated pattern is not just intra-, but also extra-cytoplasmatic. No distinctive surface characteristics, like microvilli, could be found on CTCs after filtration, which is similar to the PC 3 and LNCaP cell lines that were used as a control. Regarding their shape, $100 \%$ of CTCs from a single patient ( 28 out of 28 ) appeared to be spheroid to ovoid, probably due to the filtration procedure. Tubular or different shaped CTCs either passed through the filters together with the smaller CTCs or appeared to be spherical once captured on the microsieves.

\subsection{Discussion}

CTC load is associated with relatively poor prognosis in patients with metastatic cancer, and can be used to monitor the efficiency of a therapy ${ }^{5-9,24}$. CTC data has been generated mainly by the Cellsearch platform, but new technologies are emerging for 
CTC isolation ${ }^{10-13}$. Each technique has a different underlying principle, resulting in CTCs with selected characteristics. Consequently, the CTC identification can differ between the different existing techniques, raising the question whether phenotype differences matter. Size-based isolation techniques, like the microsieves, miss CTCs of sizes smaller than the selected pore diameter, whereas affinity-based techniques, such as the EpCAM-based CellSearch system, will miss the EpCAM- or EPCAM $\mathrm{CTCs}^{25}$. After immunomagnetic enrichment, the presence of not only CTCs with "intact" morphology, but also those undergoing apoptosis ${ }^{26-29}$ as well as tumorderived extracellular vesicles ${ }^{18,19}$ are associated with poor clinical outcome. Here, we investigated by SEM imaging the morphology of intact and apoptotic CTCs isolated by the CellSearch system and $5 \mu \mathrm{m}$ microsieves from whole blood of CRPC patients. tdEVs were possible to be SEM imaged only when the CellSearch platform was used; in the case of microsieves, they ended up in the filtrate.

CellSearch enriched CTCs, and tdEVs had a well retained morphology and shape, but their surfaces were fully covered by the ferrofluid, hindering the study of their surfaces. The complete cell coverage can be explained by the underlying principle of Cellsearch. Briefly, $\alpha$ EpCAM ferrofluid is incubated in blood samples of cancer patients and binds to EpCAM+ CTCs and tdEVs. The ferrofluid together with the bound cells are pulled out of the tube into the CellSearch cartridge by the application of magnetic forces. The enriched objects in the cartridge are perfectly aligned to the magnetic field lines of the magnets ${ }^{21}$. The excess of ferrofluid could partially explain the dim or negative signal of cells when stained for additional surface biomarkers, like the prostate-specific membrane antigen (PSMA) ${ }^{30}$. Interestingly, the size of CTCs was very similar to the rest of leukocytes that were enriched in the CellSearch cartridge, implying that a sizebased technique would miss many CTCs that fall in the same size range as leukocytes.

On the other hand, CTCs and leukocytes found on the microsieves after whole blood filtration had either lost most of their distinct surface features or they had experienced major deterioration and membrane rupture that has not been reported before. The cell deformation and stretching could be attributed to the applied pressure of $100 \mathrm{mbar}$, the distance from the neighboring pores $(9 \mu \mathrm{m}$ in vertical and diagonal axes and 19 $\mu \mathrm{m}$ in horizontal axis), and the pore size of $5 \mu \mathrm{m}$. Cote et al. have also highlighted the importance of a strong cell fixative before blood filtering ${ }^{15}$. The provider of the used microsieves, VyCAP, suggests that blood samples should be collected in Transfix preservative to ensure improved cell morphology, implying that that Cellsave used here for one to two day(s) is probably inadequate for optimal cell preservation. Lower pressure, more pores, and longer distances between them are parameters that could also improve the final cell morphology. Their further investigation is highly recommended mainly in cases where CTCs are isolated from EDTA blood and need to remain viable or be expanded for studies of their secretome or drug screening. 
It is noteworthy to mention that in case of the CellSearch isolated CTCs, when ferrofluid was not covering the cell surface, some granular structures could be observed on the CTC surfaces, as indicated by arrows in Figures 5.4 and S5.1. A speckled CK pattern in the respective immunofluorescence images would confirm the reorganization of their cytoskeleton and their early stage of apoptosis ${ }^{17,31,32}$, whereas a fragmented nucleus would even indicate advanced apoptosis ${ }^{31}$. However, in many CTCs where the punctuated CK is clearly distinguishable, the cells are fully covered by the ferrofluid, hindering the observation of blebs on the cells. In other cases, the cells, even if they had a homogeneous cytokeratin staining, had spheroid globules on their surfaces, raising the question of membrane blebbing preceding the reorganization of the keratin filament network. Alternatively, these globules could be secreted tdEVs or EVs that ended up by coincidence at the same location (Panels B and C of Figure S5.1). Interestingly, the SEM imaged blebbing of CTCs found on the microsieves corresponds to the condensed CK granular structures of the respective immunofluorescence images. That suggests that the membrane blebbing during apoptosis and the speckled CK occur simultaneously and that the observed cleaved CK pattern is not only intracytoplasmatic, but also extra-cytoplasmatic.

We here defined tdEVs in CRPC patients as EpCAM+, DNA-, CD45-, CK+ particles obtained after centrifugation of the blood samples of patients at $800 \times \mathrm{g}$ for $10 \mathrm{~min}$ and their further processing on the CellSearch system. As a consequence of the blood centrifugation, the majority of isolated tdEVs have a diameter above $1-2 \mu \mathrm{m}$. Our previous results showed that the presence of these tdEVs isolated by the Cellsearch are strongly associated with the clinical outcome of CRPC patients similarly to the $\mathrm{CTCs}^{18,19}$. Importantly, these tdEVs are rarely found in healthy donors and, in that case, their frequencies are significantly lower compared to the respective ones in CRPC patients (median value of 8 in 16 healthy donors and median value of 116 in 84 CRPC patients) ${ }^{19}$. Vagner et al. ${ }^{33}$ and Minciacchi et al. ${ }^{34}$ have demonstrated that large oncosomes of a diameter above $1 \mu \mathrm{m}$ can be found in the circulation of advanced prostate cancer patients, and constitute a separate subclass of tumor-derived extracellular vesicles that carry most of the circulating tumor DNA, reflecting the genetic aberrations of the tumor of origin. These large tdEVs do not express CD81 and CD63, which are common exosome markers, and they have a distinct protein cargo ${ }^{34}$. CK18 is one of the significantly increased proteins expressed in that class, which is also supported by our findings. Some of these tdEVs are expected to be apoptotic bodies secreted by either the CTCs undergoing apoptosis or the tumor itself. Larson et al. ${ }^{17}$ categorized EpCAM+, CK+ events into three different categories after the inclusion of M30 staining, which binds to an epitope accessible after caspase-cleaved CK18. The three classes were "intact" CTCs, CTCs "undergoing apoptosis", and "CTC fragments" (DAPI-, CK+, CD45-, M30+, or M30-). "CTC fragments" could nowadays be further classified to tumor-derived apoptotic bodies (DAPI-, CK+, CD45-, M30+) and tumor derived microvesicles (DAPI-, CK+, CD45-, M30-). Interestingly, no clear pattern 
could be observed in the different patient samples shown: One patient had only $10 \%$ of big tdEVs positive for M30, while another one had $85 \%$ of them positive for $\mathrm{M} 30$. Nevertheless, EVs have a wide size range, with the majority of them constituting the exosome subclass with a diameter below $200 \mathrm{~nm}^{35,36}$; hence, most of the tdEVs are supposed to end up in the plasma fraction of the patient samples, which is not processed by the Cellsearch system. Processing plasma of CRPC patients with the CellSearch system could reveal what the actual proportion of smaller tdEVs is. Preliminary results (data not shown) indicate that isolation of tdEVs from plasma of patients is indeed feasible using the CellSearch, but further investigation is needed. It should be taken into consideration that the smaller size tdEV populations may express very low amounts or even no EpCAM on their membranes depending on their biogenesis. Ferrofluid conjugated with multiple antibodies recognizing more than one tumor- or epithelial- specific surface biomarkers (e.g. $\alpha$ EpCAM together with $\alpha$ Caveolin-1 and $\alpha$ PSMA) and incubated in the plasma of patient samples and downstream characterization of the isolated EVs could provide higher tdEV capture yields and more insights about the cells of origin. There are some SEM images of EVs in the literature ${ }^{37,38}$; however, the identity of the depicted particles is always doubtful since no other correlative technique is being used to confirm the chemical composition or the surface marker expression of the imaged EVs in a single level.

Herein, the fluorescence imaging of tdEVs with $\alpha \mathrm{CK}-\mathrm{PE}$ staining and their capture by $\alpha$ EpCAM ferrofluid, which are both epithelial specific markers, with $\mathrm{CK}$ being expressed in the interior of epithelial cells and EpCAM on their surface, confirm their epithelial/tumor origin. Particles of a similar size as the ones shown in Figure 5.6, captured by the $\alpha$ EpCAM ferrofluid, were also found, but they were negative for $\mathrm{CK}$, CD45, and DNA (Figure S5.3), and were not detected by the CellTracks Analyzer II. Further investigation using additional antibodies against generic membrane markers, like wheat germ agglutinin, or cell-specific antigens, such as HER2 (breast), PSMA (prostate), CD16 (leukocytes), or CD61 (platelets), could reveal the origin of these particles as already demonstrated by de Wit and Zeune ${ }^{39}$.

In conclusion, our findings suggest that CTCs isolated by the CellSearch system retain their cell morphology and have different shapes and sizes. However, the coverage of most of their surfaces by the $\alpha$ EpCAM ferrofluid prevents their detailed morphological characterization, while at the same time, raises questions about the staining efficiency of additional surface markers expressed in low levels mainly after CTC isolation. Conversely, CTCs isolated purely by filtration through $5 \mu \mathrm{m}$ microsieves experienced major cell deformation due to several factors, including the applied pressure, limited number of pores, and close pore proximity. In studies that CTCs need to be viable after their isolation and get expanded, e.g. studies of CTC secretome, drug efficiency testing, or for understanding the underlying biology of tumor growth and mutational status, the CTC profile kit is available from the CellSearch system, where CTCs can be immunomagnetically isolated from EDTA blood 
samples without being further fixed, permeabilized and stained. In the case of filtration, less harsh handling is required to be further evaluated, such as lower applied pressure, or incorporated microsieves within microfluidics to ensure CTC viability.

Finally, the SEM imaging of the CellSearch cartridges also allowed imaging of EpCAM+, $\mathrm{CK}+, \mathrm{CD}_{45}-$, DNA- tdEVs isolated from the blood cell fraction of the patient samples, confirming their three-dimensional shape and that these are not just cell membrane fragments or antibody artefacts. The existence of particles of similar size and shape that were not detected by immunofluorescence imaging because of the lack of nucleus and CK, CD45 expression requires further investigation to reveal their origin.

\section{Acknowledgments}

The authors acknowledge Richard Borsboom, Christian Breukers and Aufried Lenferink for their technical support on the SEM. We would like to thank the Materials Science and Technology of Polymers department of the University of Twente for giving us access to their sputter coater JEOL JFC-1300 and the Experimental Centre for Technical Medicine (ECTM) of the University of Twente for providing us with blood samples of healthy donors. We would also like to acknowledge all the patients and healthy donors, the blood samples of whom were used for the accomplishment of the present study. 


\section{References}

1. Lambert AW, Pattabiraman DR, Weinberg RA. Emerging Biological Principles of Metastasis. Cell 2017; 168(4): 670-691; doi 10.1016/j.cell.2016.11.037.

2. Mori M, Mimori K, Ueo H, Karimine N, Barnard GF, Sugimachi K et al. Molecular detection of circulating solid carcinoma cells in the peripheral blood: the concept of early systemic disease. Int J Cancer 1996; 68(6): 739-743; doi 10.1002/(SICI)1097-0215(19961211)68:6<739::AIDIJC8>3.0.CO;2-4.

3. Cristofanilli M, Budd GT, Ellis MJ, Stopeck A, Matera J, Miller MC et al. Circulating tumor cells, disease progression, and survival in metastatic breast cancer. N Engl J Med 2004; 351(8): 781-791; doi 10.1056/NEJMoa040766.

4. Moreno JG, Miller MC, Gross S, Allard WJ, Gomella LG, Terstappen LW. Circulating tumor cells predict survival in patients with metastatic prostate cancer. Urology 2005; 65(4): 713-718; doi 10.1016/j.urology.2004.11.006.

5. Bidard FC, Peeters DJ, Fehm T, Nole F, Gisbert-Criado R, Mavroudis D et al. Clinical validity of circulating tumour cells in patients with metastatic breast cancer: a pooled analysis of individual patient data. Lancet Oncol 2014; 15(4): 406-414; doi 10.1016/S1470-2045(14)70069-5.

6. Cohen SJ, Punt CJA, Iannotti N, Saidman BH, Sabbath KD, Gabrail NY et al. Relationship of circulating tumor cells to tumor response, progression-free survival, and overall survival in patients with metastatic colorectal cancer. Journal of Clinical Oncology 2008; 26(19): 3213-3221; doi $10.1200 /$ Jco.2007.15.8923.

7. de Bono JS, Scher HI, Montgomery RB, Parker C, Miller MC, Tissing $\mathrm{H}$ et al. Circulating Tumor Cells Predict Survival Benefit from Treatment in Metastatic Castration-Resistant Prostate Cancer. Clinical Cancer Research 2008; 14(19): 6302-6309; doi 10.1158/1078-0432.Ccr08-0872.

8. Krebs MG, Sloane R, Priest L, Lancashire L, Hou JM, Greystoke A et al. Evaluation and Prognostic Significance of Circulating Tumor Cells in Patients With Non-Small-Cell Lung Cancer. Journal of Clinical Oncology 2011; 29(12): 1556-1563; doi 10.1200/Jco.2010.28.7045.

9. Hiltermann TJN, Pore MM, van den Berg A, Timens W, Boezen HM, Liesker JJW et al. Circulating tumor cells in small-cell lung cancer: a predictive and prognostic factor. Ann Oncol 2012; 23(11): 2937-2942; doi 10.1093/annonc/mds138.

10. Ferreira MM, Ramani VC, Jeffrey SS. Circulating tumor cell technologies. Mol Oncol 2016; 10(3): 374-394; doi 10.1016/j.molonc.2016.01.007.

11. Krebs MG, Metcalf RL, Carter L, Brady G, Blackhall FH, Dive C. Molecular analysis of circulating tumour cells-biology and biomarkers. Nat Rev Clin Oncol 2014; 11(3): 129-144; doi 10.1038/nrclinonc.2013.253.

12. Sharma S, Zhuang R, Long M, Pavlovic M, Kang YQ Ilyas A et al. Circulating tumor cell isolation, culture, and downstream molecular analysis. Biotechnol Adv 2018; 36(4): 1063-1078; doi 10.1016/j.biotechadv.2018.03.007.

13. Parkinson DR, Dracopoli N, Petty BG, Compton C, Cristofanilli M, Deisseroth A et al. Considerations in the development of circulating tumor cell technology for clinical use. $J$ Transl Med 2012; 10; doi Artn 13810.1186/1479-5876-10-138. 
14. Zheng S, Lin H, Liu JQ, Balic M, Datar R, Cote RJ et al. Membrane microfilter device for selective capture, electrolysis and genomic analysis of human circulating tumor cells. J Chromatogr A 2007; 1162(2): 154-161; doi 10.1016/j.chroma.2007.05.064.

15. Lin HK, Zheng S, Williams AJ, Balic M, Groshen S, Scher HI et al. Portable filter-based microdevice for detection and characterization of circulating tumor cells. Clin Cancer Res 2010; 16(20): 5011-5018; doi 10.1158/1078-0432.CCR-10-1105.

16. Sarioglu AF, Aceto N, Kojic N, Donaldson MC, Zeinali M, Hamza B et al. A microfluidic device for label-free, physical capture of circulating tumor cell clusters. Nat Methods 2015; 12(7): 685+; doi 10.1038/Nmeth.3404

17. Larson CJ, Moreno JG, Pienta KJ, Gross S, Repollet M, O'Hara S M et al. Apoptosis of circulating tumor cells in prostate cancer patients. Cytometry A 2004; 62(1):46-53; doi 10.1002/ cyto.a.20073.

18. Coumans FAW, Doggen CJM, Attard G, de Bono JS, Terstappen LWMM. All circulating EpCAM+CK+CD45-objects predict overall survival in castration-resistant prostate cancer. Ann Oncol 2010; 21(9): 1851-1857; doi 10.1093/annonc/mdq030.

19. Nanou A, Coumans FAW, van Dalum G, Zeune LL, Dolling D, Onstenk W et al. Circulating tumor cells, tumor-derived extracellular vesicles and plasma cytokeratins in castrationresistant prostate cancer patients. Oncotarget 2018; 9(27): 19283-19293; doi 10.18632/ oncotarget.25019.

20. Allard WJ, Matera J, Miller MC, Repollet M, Connelly MC, Rao C et al. Tumor cells circulate in the peripheral blood of all major carcinomas but not in healthy subjects or patients with nonmalignant diseases. Clinical Cancer Research 2004; 10(20): 6897-6904; doi Doi 10.1158/10780432.Ccr-04-0378.

21. Tibbe AG, de Grooth BG, Greve J, Dolan GJ, Rao C, Terstappen LW. Magnetic field design for selecting and aligning immunomagnetic labeled cells. Cytometry 2002; 47(3): 163-172.

22. Swennenhuis JF, Tibbe AG, Levink R, Sipkema RC, Terstappen LW. Characterization of circulating tumor cells by fluorescence in situ hybridization. Cytometry A 2009; 75(6): 520-527; doi 10.1002/cyto.a.20718.

23. Ligthart ST, Coumans FA, Bidard FC, Simkens LH, Punt CJ, de Groot MR et al. Circulating Tumor Cells Count and Morphological Features in Breast, Colorectal and Prostate Cancer. Plos One 2013; 8(6): e67148; doi 10.1371/journal.pone.0067148.

24. Coumans FAW, Ligthart ST, Uhr JW, Terstappen LWMM. Challenges in the Enumeration and Phenotyping of CTC. Clinical Cancer Research 2012; 18(20): 5711-5718; doi 10.1158/1078-0432. Ccr-12-1585.

25. de Wit S, van Dalum G, Lenferink ATM, Tibbe AGJ, Hiltermann TJN, Groen HJM et al. The detection of $\operatorname{EpCAM}(+)$ and EpCAM(-) circulating tumor cells. Sci Rep-Uk 2015; 5; doi ARTN 1227010.1038/srep12270.

26. Deutsch TM, Riethdorf S, Nees J, Hartkopf AD, Schonfisch B, Domschke C et al. Impact of apoptotic circulating tumor cells (aCTC) in metastatic breast cancer. Breast Cancer Res Treat 2016; 160(2): 277-290; doi 10.1007/s10549-016-3997-3. 
27. Allen JE, Saroya BS, Kunkel M, Dicker DT, Das A, Peters KL et al. Apoptotic circulating tumor cells (CTCs) in the peripheral blood of metastatic colorectal cancer patients are associated with liver metastasis but not CTCs. Oncotarget 2014; 5(7): 1753-1760; doi DOI 10.18632/ oncotarget.1524.

28. Smerage JB, Budd GT, Doyle GV, Brown M, Paoletti C, Muniz M et al. Monitoring apoptosis and $\mathrm{Bcl}-2$ on circulating tumor cells in patients with metastatic breast cancer. Molecular Oncology 2013; 7(3): 680-692; doi 10.1016/j.molonc.2013.02.013.

29. Jansson S, Bendahl PO, Larsson AM, Aaltonen KE, Ryden L. Prognostic impact of circulating tumor cell apoptosis and clusters in serial blood samples from patients with metastatic breast cancer in a prospective observational cohort. Bmc Cancer 2016; 16; doi ARTN 433 10.1186/ s12885-016-2406-y.

30. Gorges TM, Riethdorf S, von Ahsen O, Nastal YP, Rock K, Boede M et al. Heterogeneous PSMA expression on circulating tumor cells: a potential basis for stratification and monitoring of PSMA-directed therapies in prostate cancer. Oncotarget 2016; 7(23):34930-34941; doi 10.18632/ oncotarget.9004.

31. Caulin C, Salvesen GS, Oshima RG. Caspase cleavage of keratin 18 and reorganization of intermediate filaments during epithelial cell apoptosis. J Cell Biol 1997; 138(6): 1379-1394.

32. Dong QM, Ling C, Zhao L. Immunofluorescence analysis of cytokeratin $8 / 18$ staining is a sensitive assay for the detection of cell apoptosis. Oncol Lett 2015; 9(3): 1227-1230; doi 10.3892/ ol.2015.2856.

33. Vagner T, Spinelli C, Minciacchi VR, Balaj L, Zandian M, Conley A et al. Large extracellular vesicles carry most of the tumour DNA circulating in prostate cancer patient plasma. $J$ Extracell Vesicles 2018; 7(1): 1505403; doi 10.1080/20013078.2018.1505403.

34. Minciacchi VR, You S, Spinelli C, Morley S, Zandian M, Aspuria PJ et al. Large oncosomes contain distinct protein cargo and represent a separate functional class of tumor-derived extracellular vesicles. Oncotarget 2015; 6(13): 11327-11341; doi 10.18632/oncotarget.3598.

35. Varga Z, Yuana Y, Grootemaat AE, van der Pol E, Gollwitzer C, Krumrey M et al. Towards traceable size determination of extracellular vesicles. J Extracell Vesicles 2014; 3; doi 10.3402/ jev.v3.23298.

36. van der Pol E, Boing AN, Gool EL, Nieuwland R. Recent developments in the nomenclature, presence, isolation, detection and clinical impact of extracellular vesicles. J Thromb Haemost 2016; 14(1): 48-56; doi 10.1111/jth.13190.

37. Wu YT, Deng WT, Klinke DJ. Exosomes: improved methods to characterize their morphology, RNA content, and surface protein biomarkers. Analyst 2015; 140(19): 6631-6642; doi 10.1039/ c5anoo688k.

38. Kondratov K, Petrova T, Mikhailovskii VY, Ivanova A, Kostareva A, Fedorov A. A study of extracellular vesicles isolated from blood plasma conducted by low-voltage scanning electron microscopy. Cell and Tissue Biology 2017; 11(3): 181-190.

39. de Wit S. Circulating Tumor Cells and Beyond. Borne, the Netherlands: Medical Cell BioPhysics, University of Twente; 2018 . 


\section{Supplementary Data}
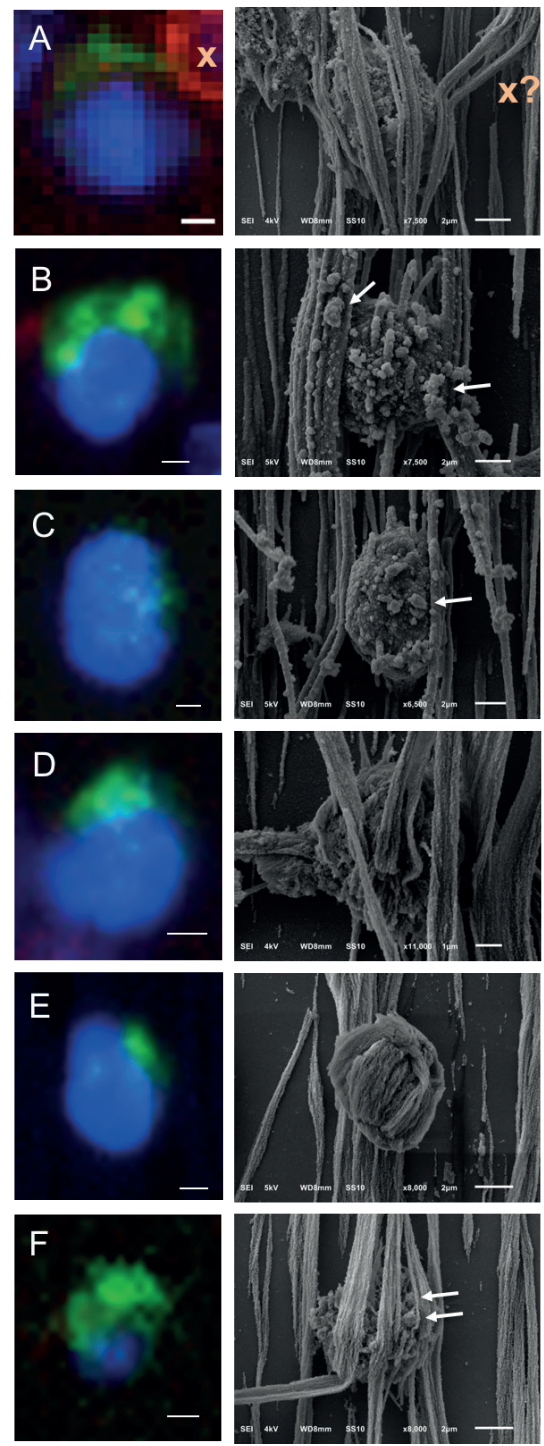
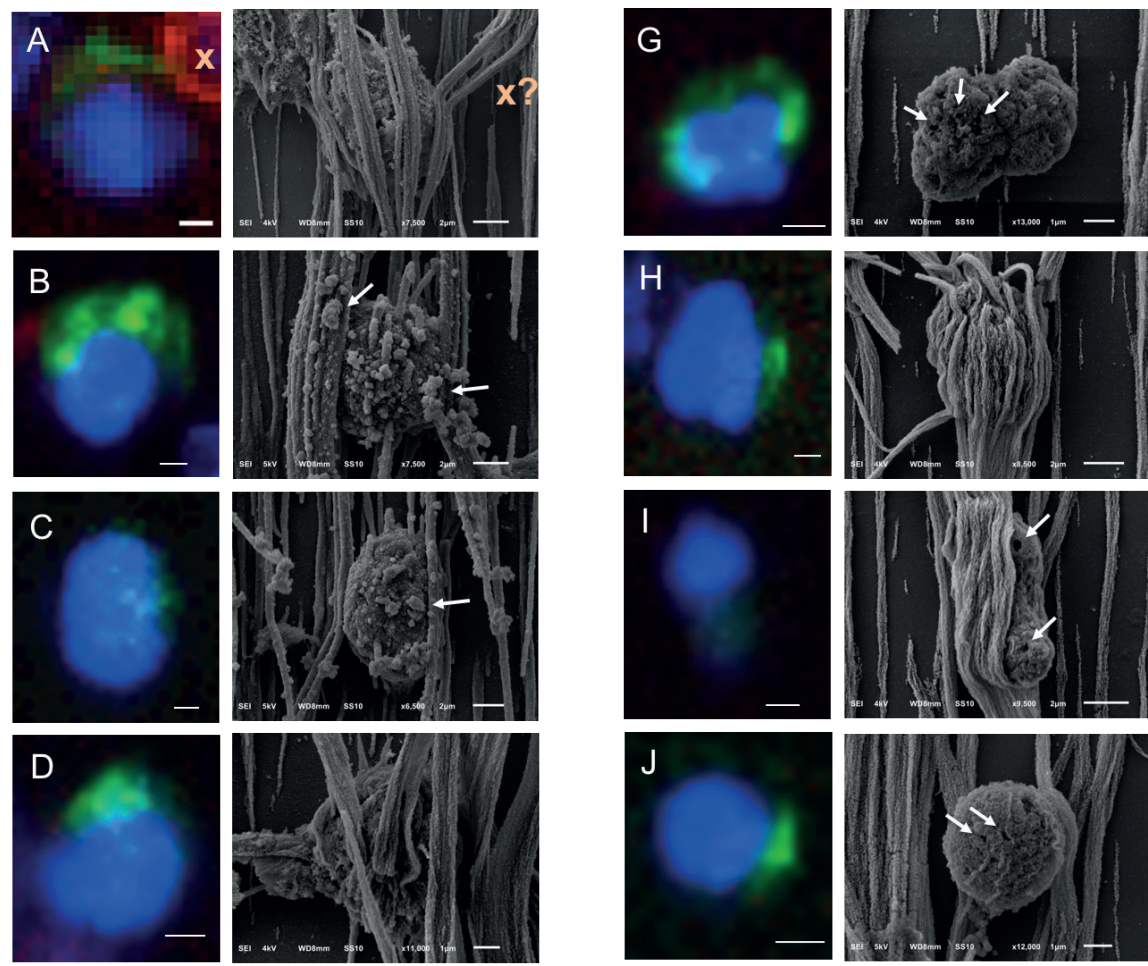

Supplementary Figure S5.1. Gallery of additional correlated fluorescence and SEM images of CTCs of a CRPC patient isolated by the CellSearch system. Nucleus (DNA) is represented by blue, CD45 by red and CK by green. Arrows are pointing at vesicles found beneath (Panel F) or on top (Panels B and C) of the ferrofluid covering the CTCs. Some CTCs appear to have sponge-like surfaces with visible holes as pointed by arrows (Panels G, I and J). Few cells (like " $x$ " in Panel A) could not be relocated by SEM, because they were lost during the dehydration/drying procedure. In other cases, additional objects (like "y" in Panel L) that were invisible by fluorescence could be visualized by SEM. Scale bars of fluorescence images indicate $2 \mu \mathrm{m}$.
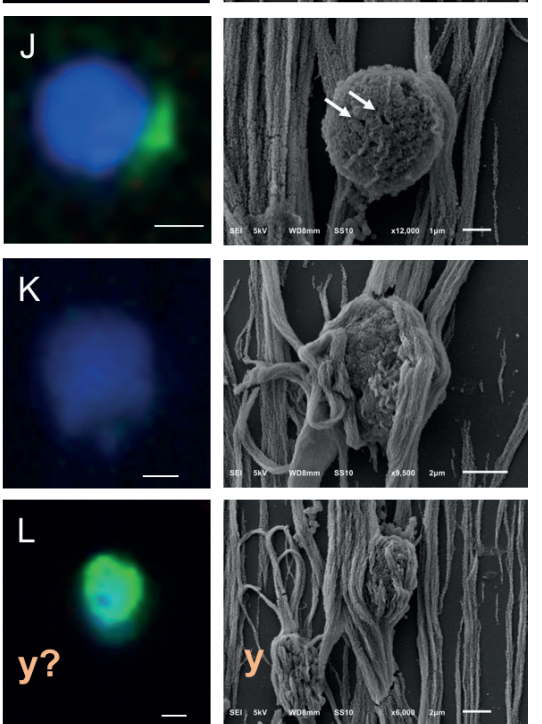


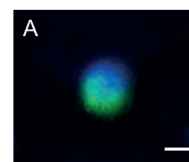

B
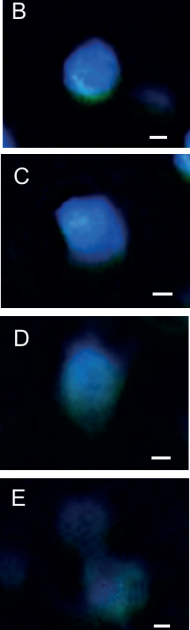

F
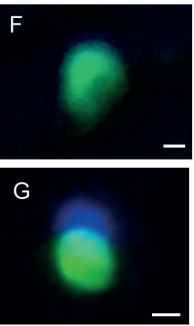

$\mathrm{H}$
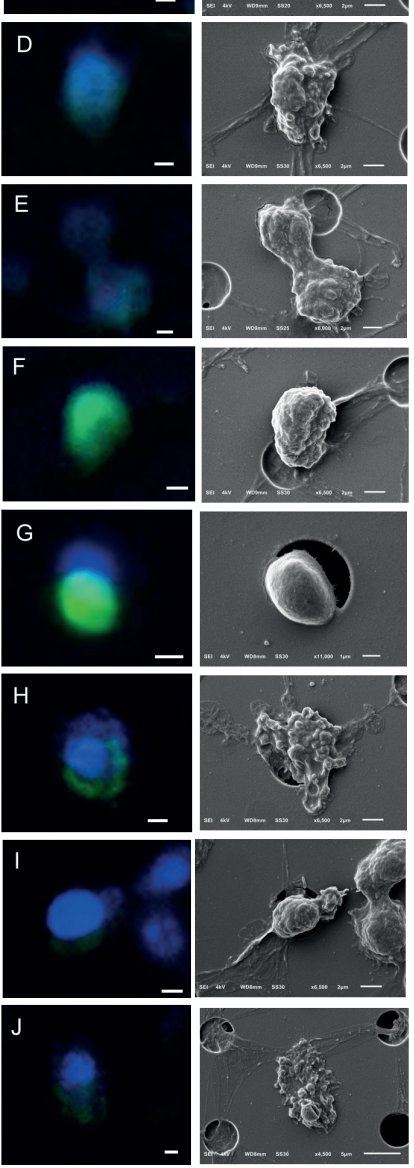
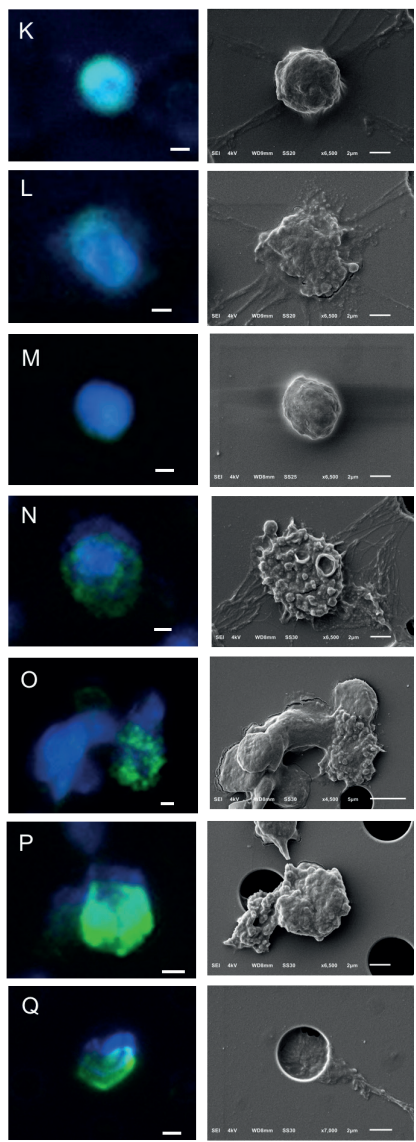

$\mathrm{R}$
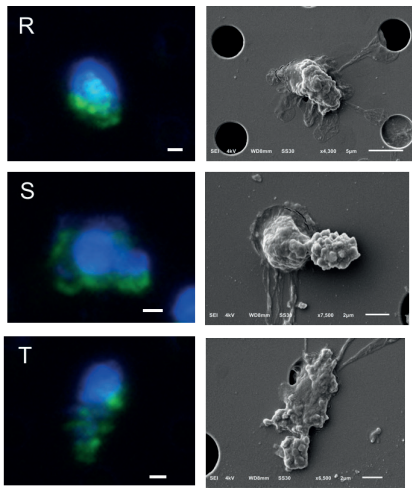

Supplementary Figure S5.2. Gallery of additional correlated fluorescence and SEM images of CTCs of a CRPC patient isolated by whole blood filtration using $5 \mu \mathrm{m}$ microsieves. Nucleus (DNA) is represented by blue and CK by green. CTCs were found on (Panels A-D, F-K, M-P, R-T), inside (Panels J, L, Q) and in between (Panel E) the pores. Apoptotic CTCs with punctuated CK pattern appeared to have blebs on their surfaces (Panels H, J, N, O, T). Scale bars of fluorescence images indicate $2 \mu \mathrm{m}$. 

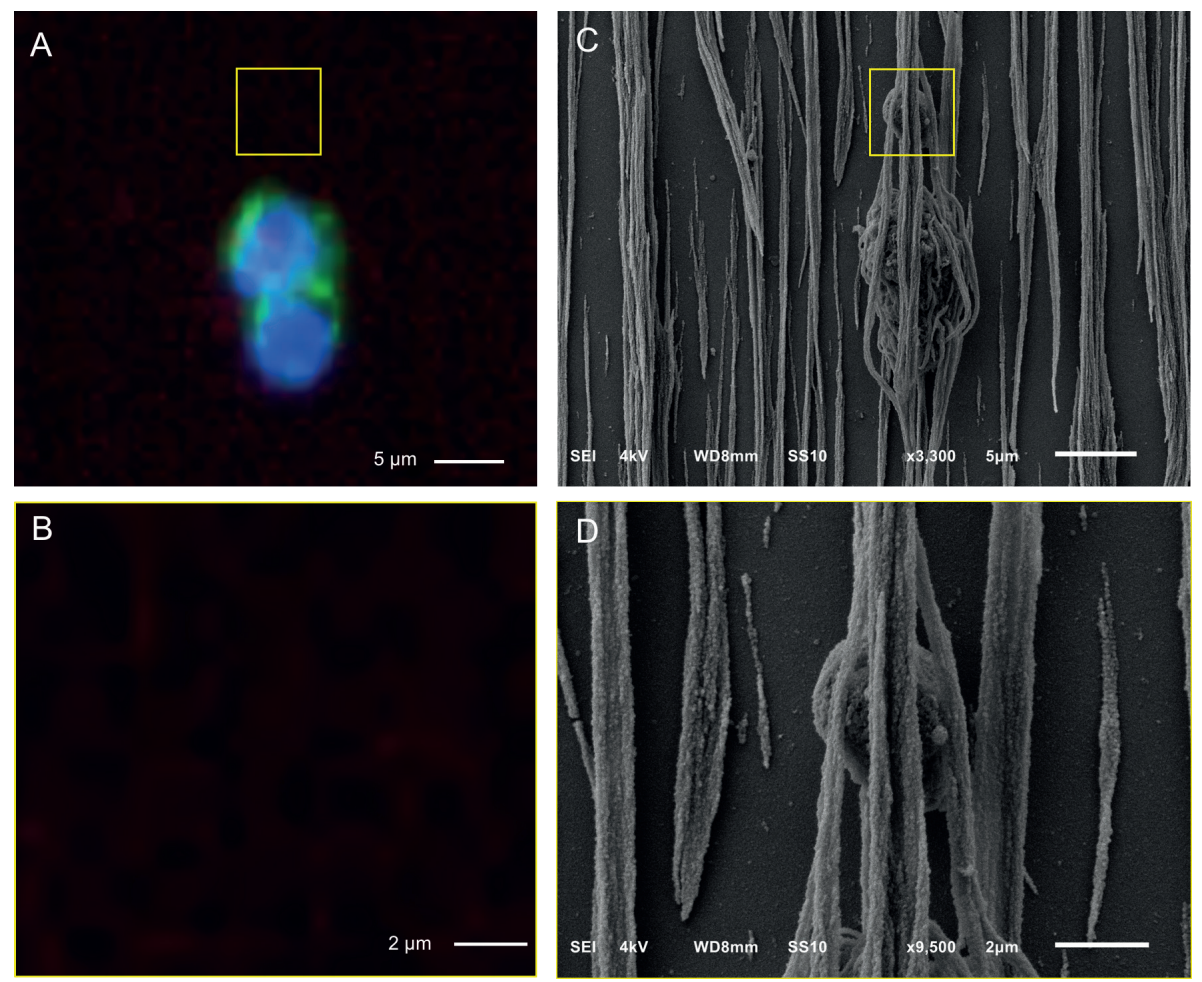

Supplementary Figure S5.3. SEM imaging (Panel C) of a CTC and an undetected by fluorescence (Panel A) particle isolated by the Cellsearch from the blood of a CRPC patient. The particle has a similar size to tdEV, but does not express CK (represented by green). Nucleus (DNA) is represented by blue. Magnified fluorescence and SEM images of the enclosed by the yellow square particle are shown in panels B and D respectively. 


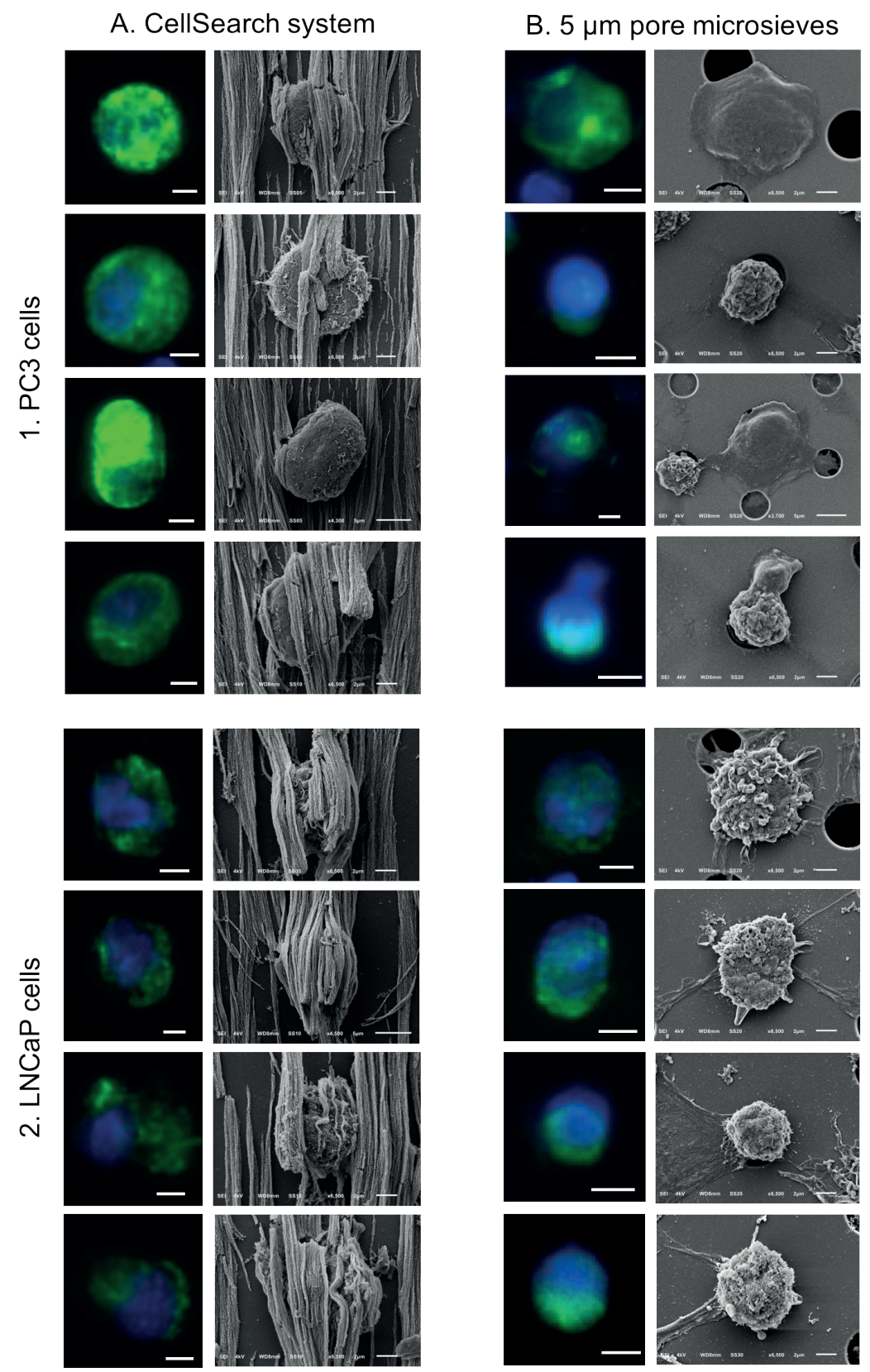

Supplementary Figure S5.4. Correlated fluorescence and SEM images of spiked 1. PC3 and 2. LNCaP cells isolated by the Cellsearch system (Panel A) or whole blood filtration using $5 \mu \mathrm{m}$ microsieves (Panel B). Nucleus (DNA) is represented by blue and CK by green. 20-30 different cells from each condition were SEM imaged, but only 4 are presented here. Scale bars in the fluorescence images indicate $4 \mu \mathrm{m}$. 
A. With big vesicles
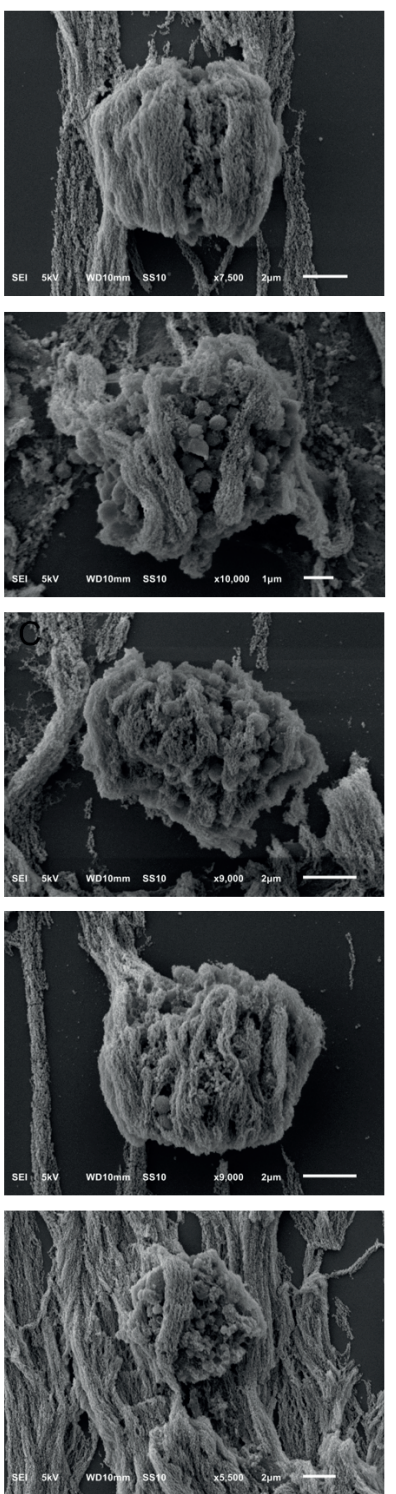

B. With small vesicles
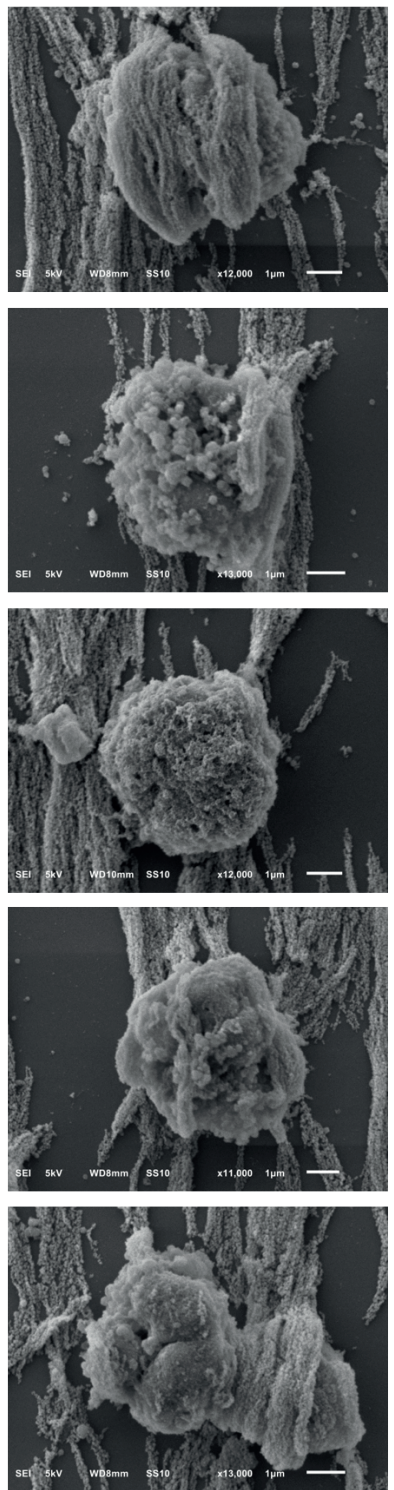

C. With no vesicles
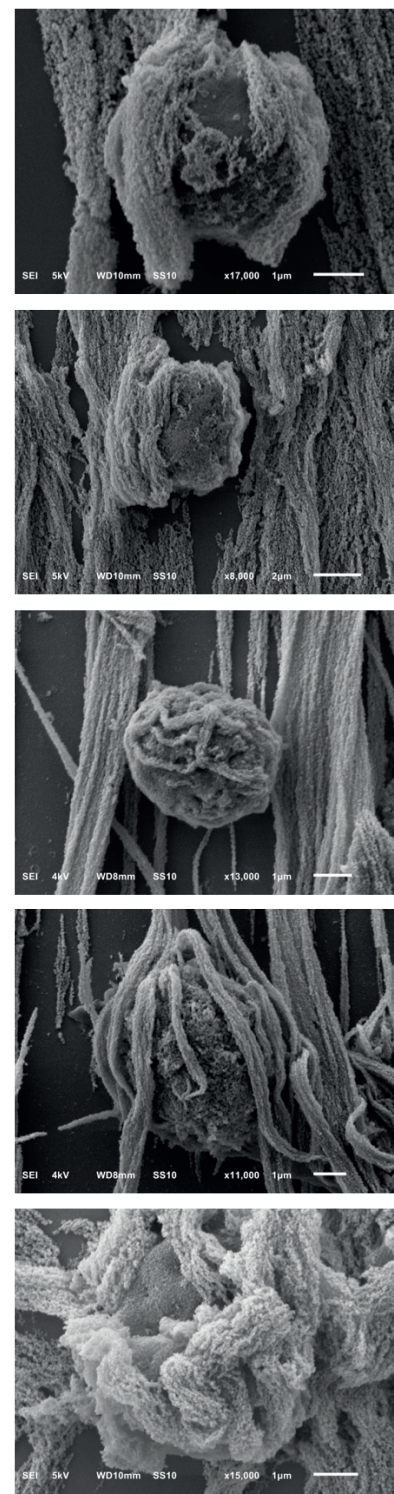

Supplementary Figure S5.5. SEM images of 15 leukocytes (out of 85 that were SEM imaged) isolated by the CellSearch from blood samples of 3 cancer patients. 15\% (13/85) of the isolated leukocytes that were SEM imaged bore big vesicles (Panel A) on their surfaces that were not observable in case of CTCs. 55\% (47/85) had smaller vesicles (Panel B) on their surfaces and 30\% (25/85) had no vesicles (Panel C) at all on their surfaces. 
A. VyCAP protocol
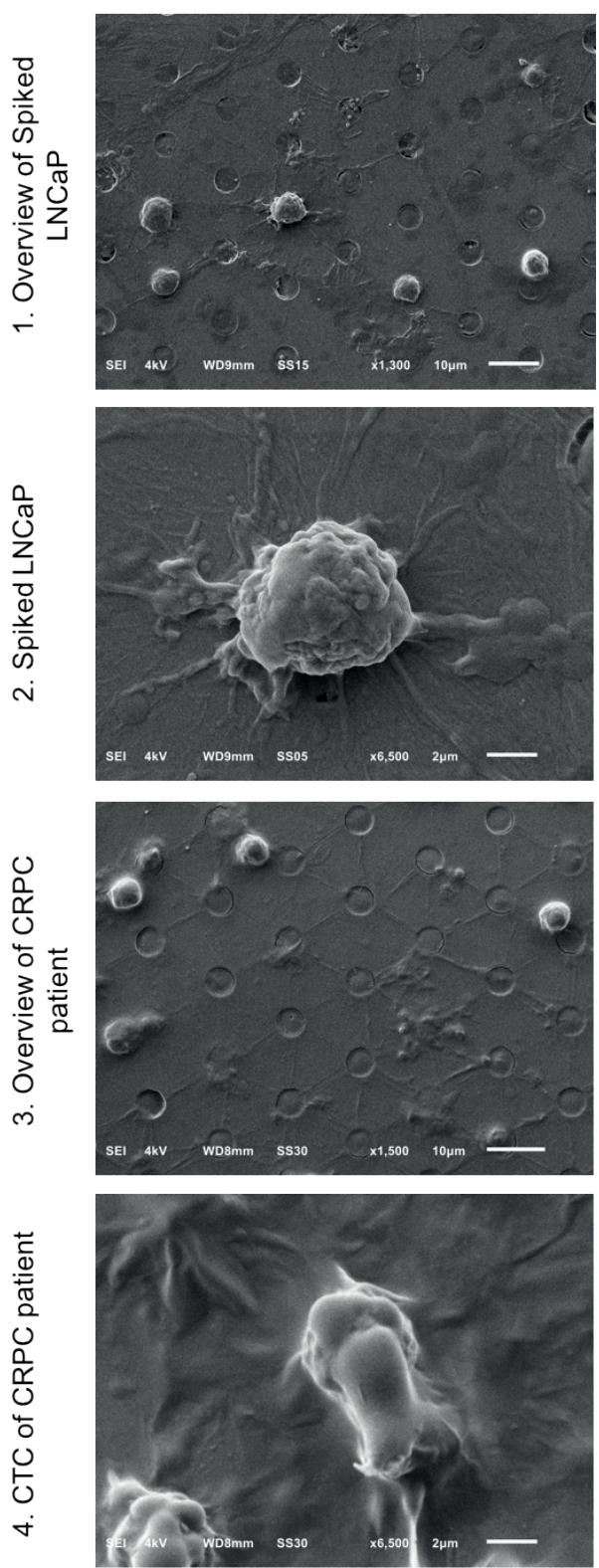

B. Followed protocol
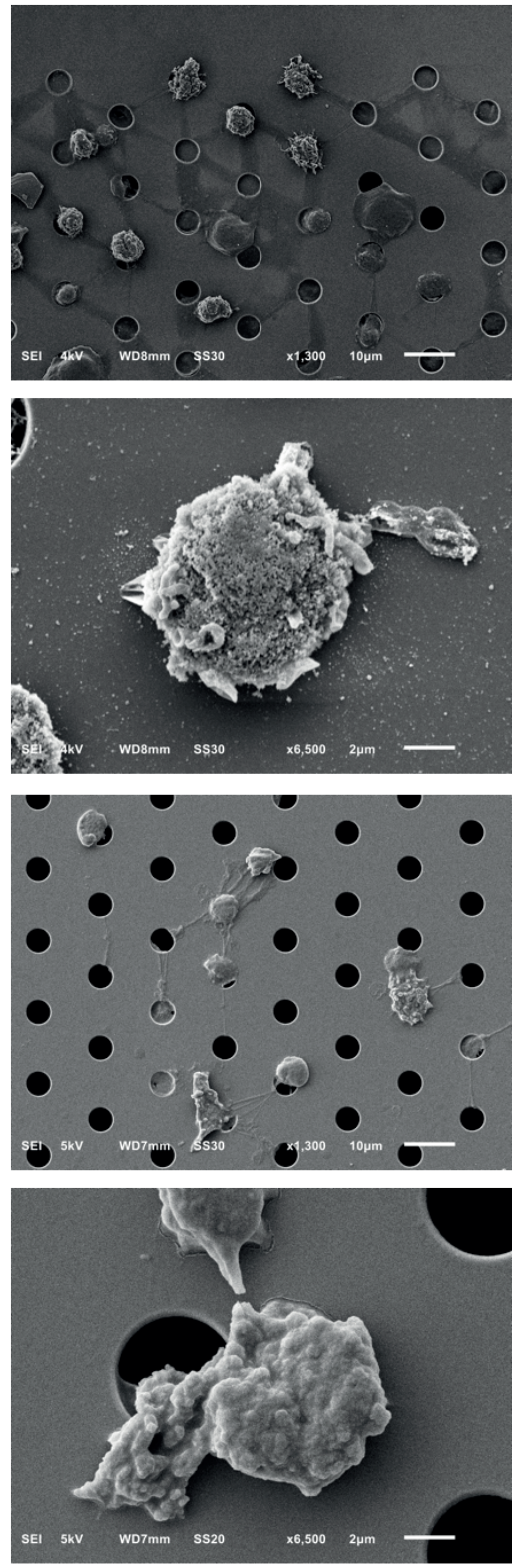

Supplementary Figure S5.6. Comparison of A. manufacturer's (VyCAP) and B. followed protocol for the permeabilization and fixation of the isolated cells/CTCs through $5 \mu \mathrm{m}$ pore microsieves. Both protocols were applied using spiked samples (Panels 1-2) and CRPC patient (Panels 3-4) samples. VyCAP protocol resulted in higher cell background during the SEM imaging that hindered the imaging of individual CTCs. 


\title{
Chapter 6
}

\section{HER2 expression on tumor-derived Extracellular Vesicles and Circulating Tumor Cells in metastatic breast cancer}

Afroditi Nanou, Leonie L. Zeune, Francois-Clement Bidard, Jean-Yves Pierga, Leon

W.M.M. Terstappen

Submitted for publication.

\begin{abstract}
Tumor-derived extracellular vesicles (tdEVs) and circulating tumor cells (CTCs) in blood of metastatic cancer patients associate with poor outcome. In this study, we explored the human epidermal growth factor receptor 2 (HER2) expression on CTCs and tdEVs of metastatic breast cancer patients. Blood samples from 98 patients were originally processed with the Cellsearch ${ }^{\circledR}$ system using the CTC kit and anti-HER2 as an additional marker in the staining cocktail. CTCs and tdEVs were automatically enumerated from the generated CellSearch image datasets using the open-source ACCEPT software. The inclusion of anti-HER2 increased the percentage of informative samples with $\geq 1$ detectable CTC and tdEV from $89 \%$ and $99 \%$ to 95 and $100 \%$, respectively. CTCs and tdEVs were subdivided based on their cytokeratin (CK) and HER2 phenotype into $\mathrm{CK}+\mathrm{HER} 2-$, $\mathrm{CK}-\mathrm{HER} 2+$ and $\mathrm{CK}+\mathrm{HER} 2+$. Inter- and intra- patient heterogeneity was found regarding the phenotype of CTCs and tdEVs with the majority of patients having all different subclasses present. CK- CTCs and tdEVs correlated equally well with clinical outcome as CK+ CTCs and tdEVs. Use of $\geq 7 \% \mathrm{HER} 2+\mathrm{CK}+\mathrm{tdEVs}$ can predict HER2 expression of the tissue with $74 \%$ sensitivity and specificity, whereas the respective use of $\geq 23 \% \mathrm{HER} 2+\mathrm{CK}+\mathrm{CTCs}$ leads to $65 \%$ sensitivity and $66 \%$ specificity. HER2 can be detected on CTCs and tdEVs not expressing CK, which have similar clinical relevance to CTCs and tdEVs expressing CK. tdEVs perform better than CTCs in predicting the HER2 status of the tissue.
\end{abstract}




\subsection{Introduction}

The peripheral blood load of Circulating Tumor Cells (CTCs) is associated with poor outcome in metastatic cancers ${ }^{1,2}$. Once present, CTCs can be used as a real-time liquid biopsy through determination of the presence of treatment targets, such as the human epidermal growth factor receptor 2 (HER2) ${ }^{3}$. The reliability of such an assessment increases with the number of CTCs available. The development of the open-source ACCEPT software permitted the automated enumeration of objects in the fluorescence images and the assessment of treatment targets on $\mathrm{CTCs}^{4}$ eliminating inter- and intra-operator variations and leading to a more objective consensus ${ }^{5}$. In metastatic breast cancer, $\sim 50 \%$ of patients have $\geq 5$ CTCs detected with the CellSearch system and the percentage of patients with $\geq 10$ and $\geq 100$ CTCs decreases rapidly ${ }^{1}$. Large tumorderived Extracellular Vesicles (tdEVs) are co-isolated with CTCs and are present in an order of magnitude higher frequencies than CTCs ${ }^{6,7}$; thus, they can increase the number of "readable" patient samples for the assessment of therapeutic targets. In this study, we evaluated the HER2 expression on CK+/- CTCs and tdEVs and its association with the HER2 expression on tissue biopsies.

\subsection{Materials and Methods}

\subsubsection{CellSearch images of breast cancer patients}

Digitally stored CellSearch ${ }^{\circledR}$ (Menarini Silicon Biosystems, Huntingdon Valley PA, USA) image files from 98 metastatic breast cancer patients from a previously reported study (IC 2006-04 study) were re-analyzed ${ }^{3,8}$. The samples had been processed with the Cellsearch using the CTC kit, as previously described ${ }^{6}$ including an additional fluorescein (FITC)-conjugated monoclonal antibody recognizing HER2 (clone Her81) in the staining mixture.

\subsubsection{Classification and automated enumeration of CTCs and tdEVs using ACCEPT}

For the automated CTC and tdEV count enumeration, the digitally stored fluorescence image files were processed with the Automated CTC Classification, Enumeration and PhenoTyping (ACCEPT) software v1.1 (http://github.com/LeonieZ/ACCEPT) using the "Full Detection" function.

\subsubsection{Statistical analysis}

Statistical analysis was performed in IBM SPSS Statistics v24.0 (SPSS Inc., Chicago, IL, USA) and MedCalc v19 (MedCalc Software, Ostend, Belgium). For the matched CTC and tdEV counts of the same subclass, a two-tailed Spearman's Rho test was performed to evaluate their relation through a monotonic function and the non- 
parametric Wilcoxon Signed Ranks test was used to test the equality of their distributions. The non-parametric Mann-Whitney U test was used to compare the distributions for the absolute and relative frequencies of CTCs and tdEVs with different immunophenotypes. The open-source web application Cutoff Finder (http://molpath. charite.de/cutoff/) was used to calculate the hazard ratios (HRs) for overall survival (OS) including $95 \%$ confidence intervals (CIs), over a wide range of cut-off values for $\mathrm{CK}+/-\mathrm{CTCs}$ and tdEVs. Cutoff Finder uses the $\mathrm{R}$ code to provide optimization and visualization tools for cut-off determination'. Receiver Operating Characteristic (ROC) curves were used to evaluate the performance of \%HER2+ CTCs (or tdEVs) in predicting HER2+ tissue as assessed by FISH (available data for 92/98 patients). The Areas Under the Curve (AUC) were compared using the non-parametric DeLong approach $^{10}$. We determined a minimum threshold for \%HER2+CK+CTCs or tdEVs to predict a HER2+ tissue as the value that led to equal sensitivity and specificity (value that led to minimum |sensitivity-specificity|). OS was defined as the elapsed time between blood draw and death and it was available for 94/98 patients. The patients who were lost during the follow up were censored. Kaplan Meier (KM) survival curves of OS were used to compare (using the log-rank test) patients with favorable and unfavorable manual and automated CTC and tdEV counts. Cox proportional hazards regression analysis was used to determine the univariable HRs with $95 \%$ CIs of the categorical and continuous CTCs and tdEVs for OS. Continuous CTC and tdEV counts were log transformed to achieve better model fit.

\subsection{Results}

\subsubsection{ACCEPT display and automated enumeration of CTCs and tdEVs}

After the inclusion of the HER2-FITC antibody, 3 different subclasses of CTCs and tdEVs were detected in the CellSearch images (Supplementary Figure S6.1). Linear ACCEPT gates were designed (Supplementary Table S6.1) and applied in the image datasets for the automated enumeration of CTCs/tdEVs of each subclass.

The automated CTC and tdEV counts of the 98 breast cancer patients are shown in dot plots in Figure 6.1A. Comparison of the applied ACCEPT gates for CTC automated enumeration and the manual CK+ CTC counts scored by the operator in the original study showed a high correlation $\left(\mathrm{r}_{\mathrm{s}}=0.83, \mathrm{p}<0.01\right)$ and similar association to the clinical outcome (Supplementary Figure S6.2).

Whereas CK+HER- tdEVs are found in a $\sim 15$-fold higher frequency compared to CK+HER-CTCs ( $\mathrm{p}<0.01$, Wilcoxon Signed Ranks test), the distributions of CK+HER2+ and CK-HER2+ tdEVs are not significantly different compared to the respective distributions of CTCs (Wilcoxon Signed Ranks test). Furthermore, the correlation 
between CTC and tdEV subclasses was very strong only in case of CK+HER2populations $\left(r_{s}=0.84, p<0.01\right)$, whereas in case of CK-HER2+ and CK+HER2+ populations, a moderate correlation was found $\left(0.5<\mathrm{r}_{\mathrm{s}}<0.6, \mathrm{p}<0.01\right)$.

A

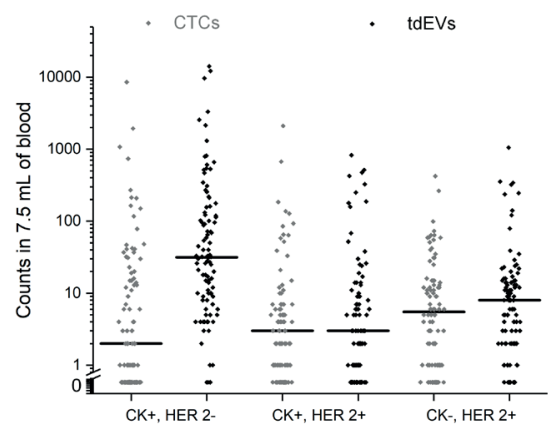

B
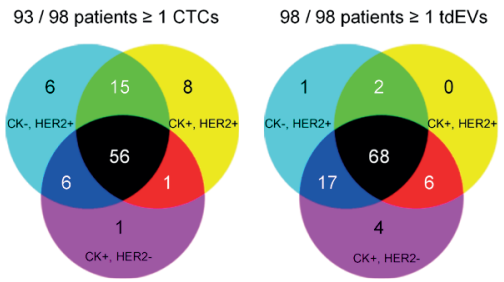

Figure 6.1. Presence and frequencies of CTC and tdEV subpopulations in metastatic breast cancer patients. in Panel A shows dot plots of different subclasses (indicated in the x-axis) of CTCs (in grey) and tdEVs (in black) in 98 metastatic breast cancer patients. Each dot corresponds to the counts of one patient. Horizontal black lines indicate median vaues. Panel B shows Venn diagrams of patients with one or more CTC (at the left) or tdEV (at the right) subclasses detected. The yellow circle corresponds to patients with CK+HER2+ CTCs (or tdEVs), the purple to patients with CK+HER2- CTCs (or tdEVs) and the cyan to patients with CK-HER2+ CTCs (or tdEVs). The overlaps of the different circles correspond to patients with 2 or more 3 CTC (or tdEV) subclasses detected. 98/98 patients had at least 1 detectable tdEV, whereas 93/98 patients had at least 1 detectable CTC.

93/98 (95\%) patients had $\geq 1$ detectable CTCs and 98/98 (100\%) of patients had $\geq 1$ tdEV. Inter- and intra- patient heterogeneity was observed regarding the CK and HER2 phenotype of CTCs and tdEVs. The Venn diagrams in Figure 6.1B show the number of patients with one or more subclasses (CK+HER2-, CK+HER2+ and CK-HER2+) of CTCs or tdEVs detected. Interestingly, the majority of patients had all three subclasses of CTCs or tdEVs present, whereas very few patients had solely one class of CTCs or tdEVs. More specifically, 56/93 (60\%) of patients had CTCs of all three immunophenotypes, followed by $22 / 93$ (24\%) of patients with CTCs of 2 different immunophenotypes and only $15 / 93$ (16\%) of patients with only 1 class of CTCs present. In case of tdEVs, the immunophenotypic heterogeneity was even better reflected with $68 / 98$ (69\%) of patients having all 3 subclasses of tdEVs present, $25 / 98$ (26\%) of them having 2 subclasses present and 5/98 (5\%) of them having one class of tdEVs present. 


\subsubsection{Association of CK+ and CK- CTCs and tdEVs with clinical outcome}

To determine whether CTCs and tdEVs lacking CK and expressing HER2 are actually cancer associated, we compared their prognostic power with CTCs and tdEVs expressing CK. For that comparison, KM plots for OS were generated (Figure 6.2) stratifying patients based on their CK+ CTCs (Panel A), CK+ tdEVs (Panel B), CKCTCs (Panel C) or CK- tdEVs (Panel D). The selected cut-offs were the minimal values leading to a dichotomization of patients with significant $\mathrm{HR}$ of OS, indicated by the green vertical lines in the respective plots of $\mathrm{HRs}$ over the range of possible $\mathrm{CK}+$ /- CTC and tdEV cut-off values (Supplementary Figure S6.3). However, the univariable Cox regression of the log transformed CK+ and CK- CTC and tdEV counts (Supplementary Table S6.2) showed that only CK+ tdEVs are significant predictors of OS.
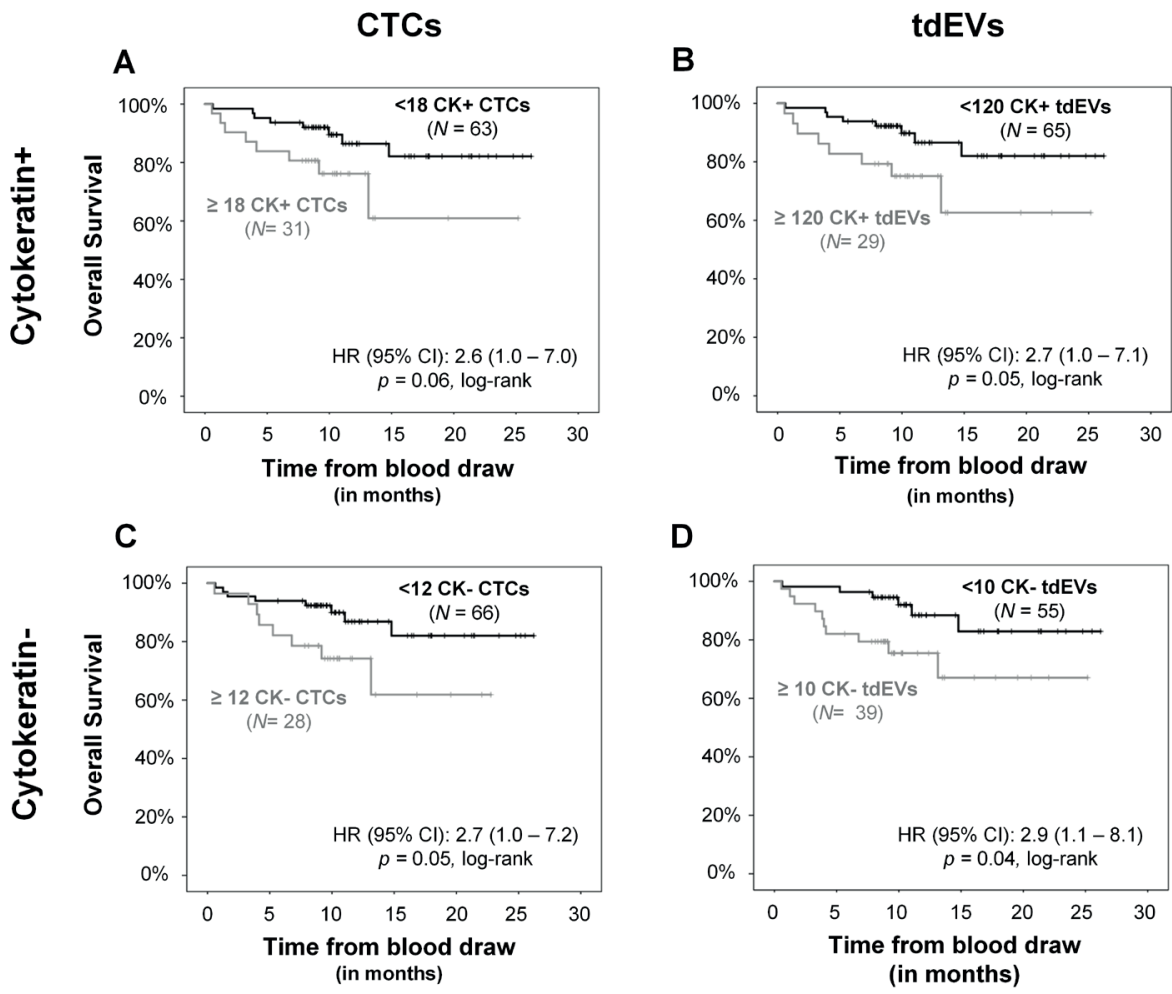

Figure 6.2. Association of $\mathrm{CK}+$ and $\mathrm{CK}-\mathrm{CTCs}$ and $\mathrm{tdEVs}$ with the clinical outcome of metastatic breast cancer patients. KM curves for OS dichotomizing patients based on their automated CK+ CTCs (Panel A) and tdEVs (Panel B) and their CK- CTCs (Panel C) and tdEVs (Panel D). CK- CTCs (or tdEVs) perform similarly to CK+ CTCs (or tdEVs) in predicting the event of the patients as shown by the patient stratification and the resulting $\mathrm{HR}$. 


\subsubsection{HER2 amplification of tissue versus HER2 expression on CTCs and tdEVs}

For 92 out of 98 patients, the HER2 status of the tissue was assessed by fluorescence in situ hybridization (FISH) with 39 patients (42\%) having HER2+ and 53 (58\%) HER2- tissue.

When we compare these 2 groups of patients in terms of their automated CTC and tdEV counts of each subclass, only CK+HER+ tdEV distribution is significantly increased $(p<0.05$, Mann-Whitney $U$ test) in HER2+ patients (Supplementary Figure S6.4, Panel B). The rest of the CTC and tdEV distributions (Supplementary Figure S6.4, Panels A and B) are not significantly different. However, the differences become more profound when comparing the 2 groups in regards to \% $\mathrm{HER} 2+\mathrm{CK}+/$, $\%$ HER2+CK+ and \% HER2+CK- CTCs and tdEVs estimated over the total CTC and tdEV counts, respectively. Patients with HER2+ tissue have significantly lower \% of $\mathrm{CK}+\mathrm{HER} 2-\mathrm{CTCs}$ and tdEVs and significantly higher \% of CK+HER2+ CTCs and tdEVs compared to patients with HER2- tissues (Supplementary Figure S6.4, Panels C and D). No statistically significant difference could be found concerning the \% of CKHER2+ CTCs and tdEVs.

To evaluate which HER2+ population is in a better concordance with the HER2+ tissue, ROC curves of the different HER2+ CTC (Figure 6.3A) and tdEV (Figure 6.3B) proportions were constructed treating $\mathrm{HER} 2+$ tissue as the classification variable. \% HER2+CK+ CTCs and tdEVs performed better as indicated by the larger AUCs $(0.69$ for CTCs and 0.79 for tdEVs, which were not significantly different, DeLong test). The asterisks in panels A and B indicate the selected threshold of \%HER2+CK+ CTCs and tdEVs, for which the test has sensitivity $\approx$ specificity. In case of CTCs, more than $23 \%$ HER2+CK+ CTCs (Figure 6.3C), could predict a HER2+ tissue with a sensitivity of $65 \%$ and specificity of $66 \%$. Likewise for tdEVs, more than $7 \% \mathrm{HER} 2+\mathrm{CK}+\mathrm{tdEVs}$ (Figure 6.3D) could predict HER2+ tissue with a sensitivity and specificity of $74 \%$. The influence of the increasing number of CTCs and tdEVs on the accuracy of the respective test, is summarized in Supplementary Table S6.3. The accuracy of $\geq 23 \% \mathrm{HER} 2+\mathrm{CK}+$ CTC test improved and reached $80 \%$ when $\geq 50$ CTCs were detected; however, only $27 / 98$ (28\%) patients accounted for that CTC load. In case of \% HER2+CK+ tdEVs, the accuracy of the test was constantly above $70 \%$ and gradually improved from $74 \%$ up to $91 \%$ with increasing tdEV counts. 


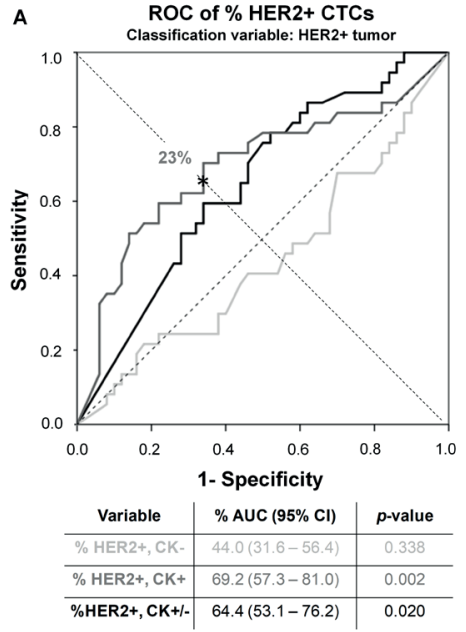

C

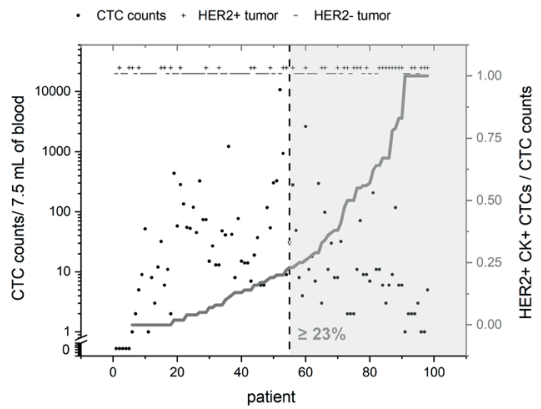

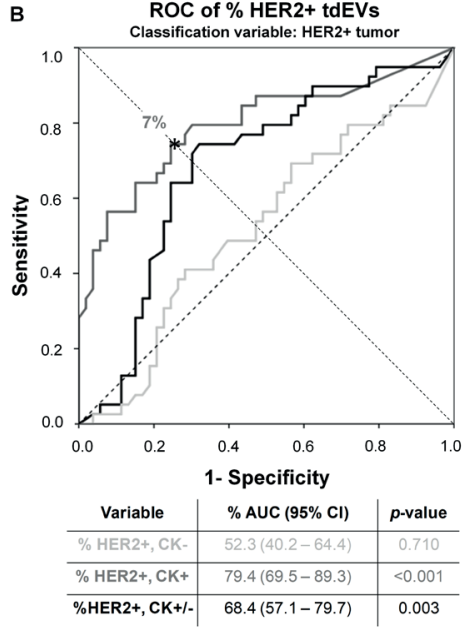

D

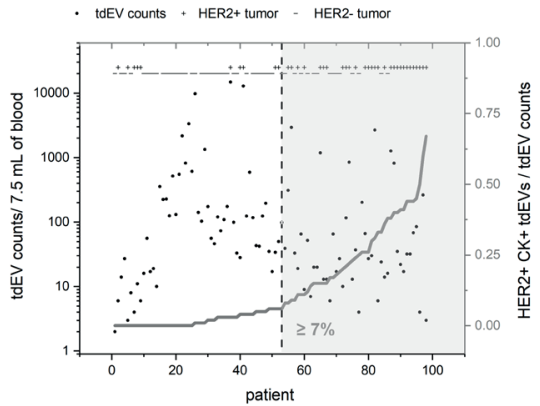

Figure 6.3. Prediction of HER2 status of tissue from CTCs and tdEVs. ROC curves of \% HER2+CK(light grey lines), HER2+CK+ (dark grey lines) and total HER2+CK+/- (black lines) CTCs (Panel A) and tdEVs (Panel B) treating HER2 + tissue (as assessed by FISH) as the classification variable. \%HER2+CK+ populations performed the best as shown by the largest AUCs. The asterisks indicate the points, where sensitivity $\approx$ specificity for CTCs and tdEVs (23\% HER2+CK+ CTCs leading to $65 \%$ sensitivity and $66 \%$ specificity; $7 \%$ HER2+CK+ tdEVs leading to $74 \%$ sensitivity and specificity). Scatter plot of total CTCs (Panel C) and tdEVs (Panel D) for each patient ( $\mathrm{x}$-axis). Samples were sorted on the \% of HER2+CK+ CTCs or tdEVs respectively indicated by the dark grey lines. On the top of the panels and along the $\mathrm{x}$-axis, the HER2 status of the tissue is indicated as positive (+) or negative (-), as evaluated by FISH. The vertical black dashed lines indicate the 23\% HER2+ CTCs (and 7\% HER2+ tdEVs) threshold, right of which the tissue of the patient could be considered as HER2+. 


\subsection{Discussion}

The HER2 is found overexpressed in around $15-30 \%$ of breast cancer cases mainly because of the HER2/neu oncogene amplification ${ }^{11,12}$. Randomized clinical trials have demonstrated its predictive and prognostic value with HER2+ breast cancer patients having a remarkably improved clinical outcome when treated with anti-HER2 therapy, such as trastuzumab or lapatinib next to chemotherapy, as compared to HER2+ patients treated with solely chemotherapy ${ }^{13-16}$. Furthermore, HER2- breast cancer patients randomized in two treatment arms with and without anti-HER2 therapy did not show any clinical benefits further supporting the predictive value of HER $2^{17}$. These observations necessitate the use of an accurate test to assess the HER2 tumor status and facilitate the clinician's treatment-decision making. The current state-of-the-art evaluates HER2 status on solid biopsies (either tumor needle biopsy or whole tumor after resection) by immunohistochemistry or/and FISH. Several guidelines have improved the accuracy of HER2 evaluation ${ }^{18}$. Nevertheless, there are cases of discorcondant immunohistochemistry and FISH results or high HER2 heterogeneity of the tumor ${ }^{19,20}$ preventing an objective consensus on HER2 status among different operators.

Non-invasive liquid biopsies have emerged to promising alternatives to solid biopsies providing clinically relevant tumor material in real-time. Increasing load of CK+ CTCs and tdEVs as identified by the CellSearch system is strongly associated with worsening progression-free and overall survival ${ }^{1,2,7,21,22}$. The Cellsearch system immunomagnetically enriches CTCs targeting the Epithelial Cell Adhesion Molecule (EpCAM); consequently, all EpCAM negative or low expressing CTCs are missed by the system. Many approaches have been introduced to overcome this limitation by enrichment of CTCs based on their physical properties such as size, density and charge ${ }^{23}$, but no obvious advantages have been demonstrated in clinical studies so far. Importantly, EpCAM ${ }^{\text {low }}, \mathrm{CK}+\mathrm{CTC}$ identified after size-based separation of EpCAM depleted blood samples did not correlate with clinical outcome in metastatic nonsmall cell lung, prostate and breast cancer ${ }^{24,25}$. In the present study, we investigated whether expression of HER2 can be assessed through tdEVs and whether CTCs and tdEVs are missed by the system due to lack of CK expression.

HER2 expression could be detected on $\mathrm{CK}+\mathrm{tdEV}$ s that were co-isolated with CTCs using the CellSearch system. The ACCEPT software allows the visualization and automated enumeration of CK- objects, which are not presented to the operator by the FDAcleared CellSearch image analysis algorithm (CellTracks Analyzer II). Interestingly, the inclusion of the HER2-FITC antibody allowed the detection of CD45-CK-HER2+ CTCs and tdEVs in the EpCAM enriched blood samples of metastatic breast cancer patients increasing the percentage of patient samples with detectable CTCs from 89 to $95 \%$. CK+ tdEVs were already present in $99 \%$ of patient samples, which increased to $100 \%$ after the inclusion of anti-HER2. 
Although we have not genetically proven that these CK- CTCs and tdEVs are indeed cancerous, their similar correlation with OS (Figure 6.2) tends to confirm that hypothesis. The observation of CK-HER2+CTCs and tdEVs raises questions about other CK-HER2- CTC and tdEV populations present in the EpCAM enriched samples. The findings of Crespo et al. that aneuploid CD45-CK-AR+ CTCs in EpCAM enriched blood samples of castration-resistant prostate cancer patients are associated with worse OS further support our hypothesis ${ }^{26}$. The detection of CK- aneuploid cells in blood samples of ovarian, breast and colorectal cancer patients has been also described by Pecot et al. ${ }^{27}$. All aforementioned studies further encourage the inclusion of additional antibodies to detect nucleated events of unkown cell lineage in the EPCAM enriched samples and decrease false negative rates of CTCs.

Interestingly, all three immunophenotypes of CTCs and tdEVs (HER2+CK-, HER2$\mathrm{CK}+$ and $\mathrm{HER} 2+\mathrm{CK}+$ ) were present in the majority of EpCAM enriched patient samples (Figure 6.1). Our next question was whereas a minimum threshold of a HER2+ population could predict the HER2 status of the tissue as a real-time liquid biopsy. The $\%$ HER2+CK+ tdEVs performed the best achieving higher sensitivity and specificity than CTCs (Figure 6.3), most likely because of the higher frequencies of tdEVs better reflecting the tumor heterogeneity. Based on that test, a patient can be affirmed to have a HER2+ tumor with at least $74 \%$ accuracy if $\geq 7 \%$ of total EpCAM+ tdEVs are $\mathrm{CK}+, \mathrm{HER} 2+$. The whole workflow from EpCAM enrichment to CTC and tdEV scoring can be done in a fully automated manner, once a blood sample of $7.5 \mathrm{~mL}$ is available. Consequently, the test is not subjected to the quality of the sample, the handling, staining methodology and the judgment of the technician. It can be performed in a timely fashion and can facilitate the treatment monitoring of the patient. Whether the HER2 assessment via a liquid biopsy (CTCs or/and tdEVs) can better predict response to anti-HER2 targeted therapies compared to the current assessment via a solid biopsy remains to be addressed.

Molecular characterization of the EpCAM+ tdEVs of patients undergoing HER2 targeted therapies at follow-up time points can contribute to better comprehend the underlying mechanism of tumor resistance to anti-HER2 treatment ${ }^{28}$, which is observed in more than $70 \%$ of HER+ breast cancer patients within a year from the initiation of the treatment ${ }^{29}$. Ciravolo et al. have already suggested a mechanism of anti-HER2 resistance by the increased binding efficiency of HER2+ exosomes to Trastuzumab in progressive HER2+ breast cancers as compared to earlier stages of breast cancer ${ }^{30}$. Another mechanism has been suggested by Al-Nedawi et al. with tdEVs transferring the oncogenic form of epidermal growth factor receptor EGFRvIII to cells without that immunophenotype ${ }^{31}$. 
In summary, the inclusion of anti-HER2 in the CellSearch assay allowed the detection of EpCAM+CK- CTCs and tdEVs with similar prognostic power as EpCAM+CK+ CTCs and tdEVs in metastatic breast cancer emerging the importance of including more detection antibodies next to CK for the identification of CK- CTCs and tdEVs. Our results enrich the already available data of "real-time" liquid biopsy and encourage the screening of tdEVs for treatment targets in clinics, since they seem to better reflect the HER2 phenotype of the tumor than CTCs opening the path towards a more rational and objective choice of the patients who will or will not be subjected to HER2 targeting therapies.

\section{Declarations}

\section{Ethics Approval and consent to participate}

The original IC 2006-04 study8 , identified as 'DGS 2006-A00523-48' (France) and 'NCT00898014' (United States), was approved by the national ethics board. The studies were performed in accordance with the Declaration of Helsinki. All individuals provided written informed consent prior to participation.

\section{Acknowledgments}

The authors acknowledge all patients and healthy donors, the blood samples of whom were used for the accomplishment of the present study. 


\section{References}

1. Miller MC, Doyle GV, Terstappen LW. Significance of Circulating Tumor Cells Detected by the CellSearch System in Patients with Metastatic Breast Colorectal and Prostate Cancer. J Oncol 2010; 2010: 617421; doi 10.1155/2010/617421.

2. Lindsay CR, Blackhall FH, Carmel A, Fernandez-Gutierrez F, Gazzaniga P, Groen HJM et al. EPAC-lung: pooled analysis of circulating tumour cells in advanced non-small cell lung cancer. Eur J Cancer 2019; 117: 60-68; doi 10.1016/j.ejca.2019.04.019.

3. Ligthart ST, Bidard FC, Decraene C, Bachelot T, Delaloge S, Brain E et al. Unbiased quantitative assessment of Her-2 expression of circulating tumor cells in patients with metastatic and non-metastatic breast cancer. Ann Oncol 2013; 24(5): 1231-1238; doi 10.1093/annonc/mds625.

4. Zeune L, van Dalum G, Decraene C, Proudhon C, Fehm T, Neubauer H et al. Quantifying HER-2 expression on circulating tumor cells by ACCEPT. Plos One 2017; 12(10): e0186562.

5. Zeune LL, de Wit S, Berghuis AMS, Ijzerman MJ, Terstappen LWMM, Brune C. How to Agree on a CTC: Evaluating the Consensus in Circulating Tumor Cell Scoring. Cytometry Part A : the journal of the International Society for Analytical Cytology 2018; 93(12): 1202-1206.

6. Nanou A, Coumans FAW, van Dalum G, Zeune LL, Dolling D, Onstenk W et al. Circulating tumor cells, tumor-derived extracellular vesicles and plasma cytokeratins in castrationresistant prostate cancer patients. Oncotarget 2018; 9(27): 19283-19293.

7. Nanou A, Miller MC, Zeune LL, de Wit S, Punt CJA, Groen HJM et al. Tumour-derived extracellular vesicles in blood of metastatic cancer patients associate with overall survival. $\mathrm{Br}$ J Cancer 2020; doi 10.1038/s41416-019-0726-9.

8. Pierga JY, Hajage D, Bachelot T, Delaloge S, Brain E, Campone M et al. High independent prognostic and predictive value of circulating tumor cells compared with serum tumor markers in a large prospective trial in first-line chemotherapy for metastatic breast cancer patients. Ann Oncol 2012; 23(3): 618-624; doi 10.1093/annonc/mdr263.

9. Budczies J, Klauschen F, Sinn BV, Gyorffy B, Schmitt WD, Darb-Esfahani S et al. Cutoff Finder: a comprehensive and straightforward Web application enabling rapid biomarker cutoff optimization. Plos One 2012; 7(12): e51862; doi 10.1371/journal.pone.0051862.

10. DeLong ER, DeLong DM, Clarke-Pearson DL. Comparing the areas under two or more correlated receiver operating characteristic curves: a nonparametric approach. Biometrics 1988; 44(3): 837-845.

11. Slamon DJ, Clark GM, Wong SG, Levin WJ, Ullrich A, McGuire WL. Human breast cancer: correlation of relapse and survival with amplification of the HER-2/neu oncogene. Science (New York, N Y) 1987; 235(4785): 177-182.

12. Gullick WJ, Love SB, Wright C, Barnes DM, Gusterson B, Harris AL et al. c-erbB-2 protein overexpression in breast cancer is a risk factor in patients with involved and uninvolved lymph nodes. British journal of cancer 1991; 63(3): 434-438.

13. Geyer CE, Forster J, Lindquist D, Chan S, Romieu CG, Pienkowski T et al. Lapatinib plus capecitabine for HER2-positive advanced breast cancer. N Engl J Med 2006; 355(26): 2733-2743; doi 10.1056/NEJMoa064320. 
14. Gianni L, Eiermann W, Semiglazov V, Manikhas A, Lluch A, Tjulandin S et al. Neoadjuvant chemotherapy with trastuzumab followed by adjuvant trastuzumab versus neoadjuvant chemotherapy alone, in patients with HER2-positive locally advanced breast cancer (the NOAH trial): a randomised controlled superiority trial with a parallel HER2-negative cohort. Lancet (London, England) 2010; 375(9712): 377-384.

15. Piccart-Gebhart MJ, Procter M, Leyland-Jones B, Goldhirsch A, Untch M, Smith I et al. Trastuzumab after adjuvant chemotherapy in HER2-positive breast cancer. The New England journal of medicine 2005; 353(16): 1659-1672.

16. Baselga J, Bradbury I, Eidtmann H, Di Cosimo S, de Azambuja E, Aura C et al. Lapatinib with trastuzumab for HER2-positive early breast cancer (NeoALTTO): a randomised, open-label, multicentre, phase 3 trial. Lancet (London, England) 2012; 379(9816): 633-640.

17. Di Leo A, Gomez HL, Aziz Z, Zvirbule Z, Bines J, Arbushites MC et al. Phase III, double-blind, randomized study comparing lapatinib plus paclitaxel with placebo plus paclitaxel as firstline treatment for metastatic breast cancer. Journal of clinical oncology : official journal of the American Society of Clinical Oncology 2008; 26(34): 5544-5552.

18. Wolff AC, Hammond MEH, Allison KH, Harvey BE, Mangu PB, Bartlett JMS et al. Human Epidermal Growth Factor Receptor 2 Testing in Breast Cancer: American Society of Clinical Oncology/College of American Pathologists Clinical Practice Guideline Focused Update. Journal of clinical oncology : official journal of the American Society of Clinical Oncology 2018; 36(20): 2105-2122.

19. Buckley NE, Forde C, McArt DG, Boyle DP, Mullan PB, James JA et al. Quantification of HER2 heterogeneity in breast cancer-implications for identification of sub-dominant clones for personalised treatment. Sci Rep 2016; 6: 23383; doi 10.1038/srep23383.

20. Nitta H, Kelly BD, Allred C, Jewell S, Banks P, Dennis E et al. The assessment of HER2 status in breast cancer: the past, the present, and the future. Pathol Int 2016; 66(6): 313-324.

21. Ligthart ST, Coumans FAW, Attard G, Cassidy AM, de Bono JS, Terstappen LWMM. Unbiased and automated identification of a circulating tumour cell definition that associates with overall survival. Plos One 2011; 6(11): e27419.

22. de Wit S, Rossi E, Weber S, Tamminga M, Manicone M, Swennenhuis JF et al. Single tube liquid biopsy for advanced non-small cell lung cancer. International journal of cancer 2019; 144(12): 3127-3137.

23. Harouaka R, Kang Z, Zheng SY, Cao L. Circulating tumor cells: advances in isolation and analysis, and challenges for clinical applications. Pharmacol Ther 2014; 141(2): 209-221; doi 10.1016/j.pharmthera.2013.10.004.

24. de Wit S, Manicone M, Rossi E, Lampignano R, Yang L, Zill B et al. EpCAM(high) and EpCAM(low) circulating tumor cells in metastatic prostate and breast cancer patients. Oncotarget 2018; 9(86):35705-35716; doi 10.18632/oncotarget.26298.

25. de Wit S, van Dalum G, Lenferink ATM, Tibbe AGJ, Hiltermann TJN, Groen HJM et al. The detection of $\operatorname{EpCAM}(+)$ and EpCAM(-) circulating tumor cells. Sci Rep-Uk 2015; 5; doi ARTN $1227010.1038 /$ srep12270. 
26. Crespo M, van Dalum G, Ferraldeschi R, Zafeiriou Z, Sideris S, Lorente D et al. Androgen receptor expression in circulating tumour cells from castration-resistant prostate cancer patients treated with novel endocrine agents. Br J Cancer 2015; 112(7): 1166-1174; doi 10.1038/ bjc.2015.63.

27. Pecot CV, Bischoff FZ, Mayer JA, Wong KL, Pham T, Bottsford-Miller J et al. A novel platform for detection of CK+ and CK- CTCs. Cancer Discov 2011; 1(7): 580-586; doi 10.1158/2159-8290. CD-11-0215.

28. Pohlmann PR, Mayer IA, Mernaugh R. Resistance to Trastuzumab in Breast Cancer. Clinical cancer research : an official journal of the American Association for Cancer Research 2009; 15(24): 7479-7491.

29. Vogel CL, Cobleigh MA, Tripathy D, Gutheil JC, Harris LN, Fehrenbacher L et al. Efficacy and safety of trastuzumab as a single agent in first-line treatment of HER2-overexpressing metastatic breast cancer. Journal of clinical oncology : official journal of the American Society of Clinical Oncology 2002; 20(3): 719-726.

30. Ciravolo V, Huber V, Ghedini GC, Venturelli E, Bianchi F, Campiglio M et al. Potential role of HER2-overexpressing exosomes in countering trastuzumab-based therapy. J Cell Physiol 2012; 227(2): 658-667; doi 10.1002/jcp.22773.

31. Al-Nedawi K, Meehan B, Micallef J, Lhotak V, May L, Guha A et al. Intercellular transfer of the oncogenic receptor EGFRvIII by microvesicles derived from tumour cells. Nat Cell Biol 2008; 10(5): 619-624; doi 10.1038/ncb1725. 


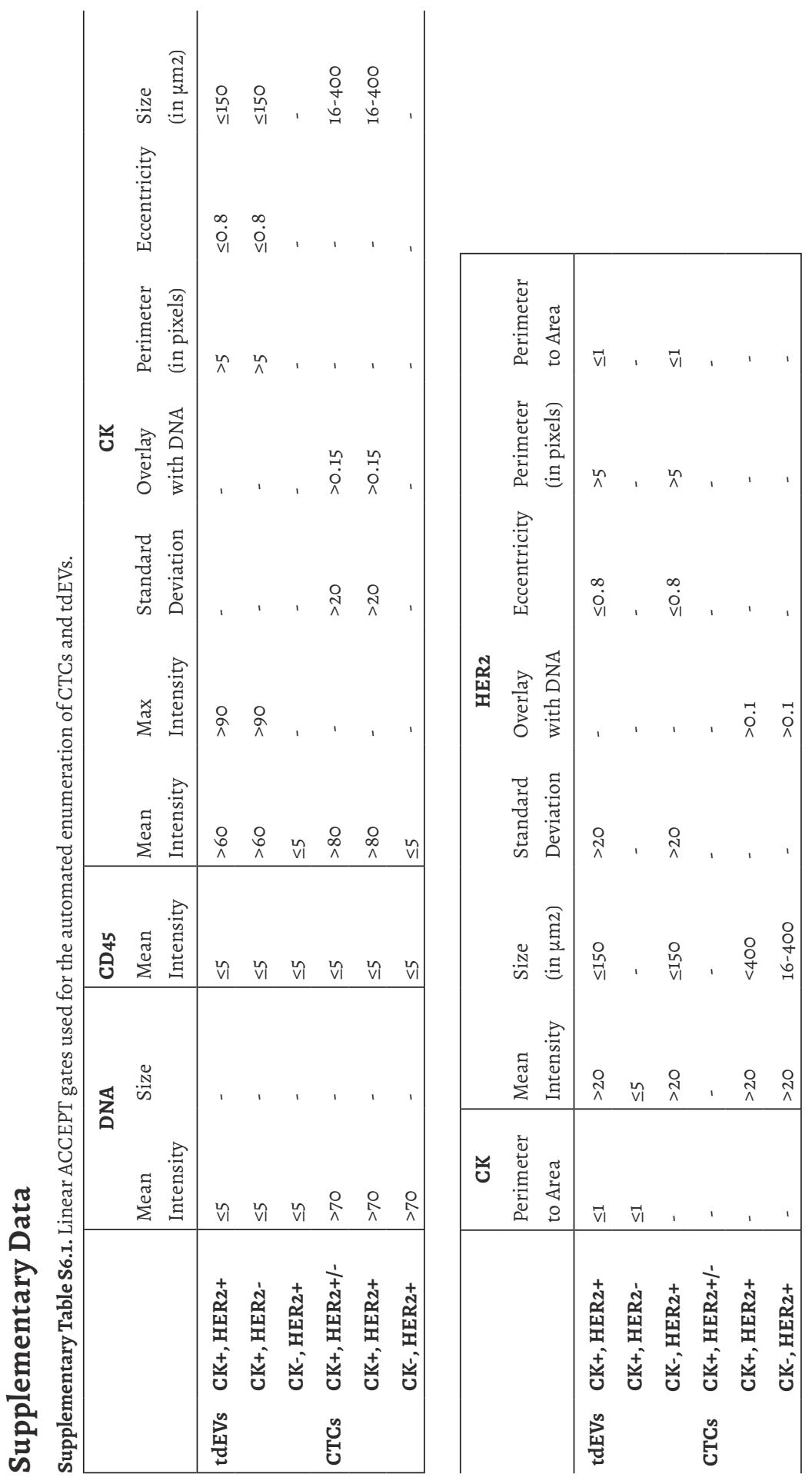


Supplementary Table S6.2. Univariable Cox regression analyses of CK+ and CK- CTCs and tdEVs after $\log$ transformation.

\begin{tabular}{|c|c|c|c|c|}
\hline & \multicolumn{2}{|c|}{ Progression-free Survival } & \multicolumn{2}{|l|}{ Overall survival } \\
\hline & HR (95\% CI) & p-value & HR ( $95 \% \mathrm{CI})$ & p-value \\
\hline $\log \left(0.1+\mathrm{CK}^{-} \mathrm{CTCs}\right)$ & $1.69(0.94-3.03)$ & 0.081 & $1.70(0.94-3.08)$ & 0.081 \\
\hline $\log \left(0.1+\mathrm{CK}^{+} \mathrm{CTCs}\right)$ & $1.41(0.90-2.21)$ & 0.131 & $1.41(0.89-2.24)$ & 0.140 \\
\hline $\log \left(0.1+\mathrm{CK}^{-} \mathrm{tdEVs}\right)$ & $1.29(0.75-2.23)$ & 0.358 & $1.32(0.75-2.32)$ & 0.345 \\
\hline $\log \left(0.1+\mathrm{CK}^{+}\right.$tdEVs $)$ & $1.65(1.01-2.70)$ & 0.044 & $1.69(1.03-2.79)$ & 0.039 \\
\hline
\end{tabular}

Supplementary Table S6.3. Sensitivity, specificity and accuracy of $\geq 23 \%$ CTCs and $\geq 7 \%$ tdEVs, double positive for $\mathrm{CK}$ and HER2, as tests to predict the HER2 status of the tissue. The accuracy increases with the total CTCs and tdEVs detected $(\geq 1,5,10,20,50,100)$ at the cost of number of eligible patients to be assessed.

\begin{tabular}{llllll}
\hline HER2+CK+ CTCs / total CTCs & Total CTCs & Patients N (\%) & sensitivity & specificity & accuracy \\
\hline & $\geq 1$ & $93(95 \%)$ & $65 \%$ & $66 \%$ & $66 \%$ \\
& $\geq 5$ & $75(77 \%)$ & $59 \%$ & $71 \%$ & $65 \%$ \\
$\geq 23 \%$ & $\geq 10$ & $53(54 \%)$ & $47 \%$ & $78 \%$ & $63 \%$ \\
& $\geq 20$ & $38(39 \%)$ & $46 \%$ & $86 \%$ & $66 \%$ \\
& $\geq 50$ & $27(28 \%)$ & $60 \%$ & $100 \%$ & $80 \%$ \\
& $\geq 100$ & $17(17 \%)$ & $67 \%$ & $100 \%$ & $84 \%$ \\
\hline HER2+CK+ tdEVs / total tdEVs & Total tdEVs & Patients N $(\%)$ & sensitivity & specificity & accuracy \\
\hline & $\geq 1$ & $98(100 \%)$ & $74 \%$ & $74 \%$ & $74 \%$ \\
& $\geq 5$ & $92(94 \%)$ & $77 \%$ & $74 \%$ & $76 \%$ \\
& $\geq 10$ & $85(87 \%)$ & $81 \%$ & $75 \%$ & $78 \%$ \\
& $\geq 20$ & $69(70 \%)$ & $79 \%$ & $77 \%$ & $78 \%$ \\
& $\geq 50$ & $48(49 \%)$ & $80 \%$ & $89 \%$ & $85 \%$ \\
& $\geq 100$ & $36(37 \%)$ & $82 \%$ & $100 \%$ & $91 \%$ \\
\hline
\end{tabular}


Circulating Tumor Cells (CTCs)
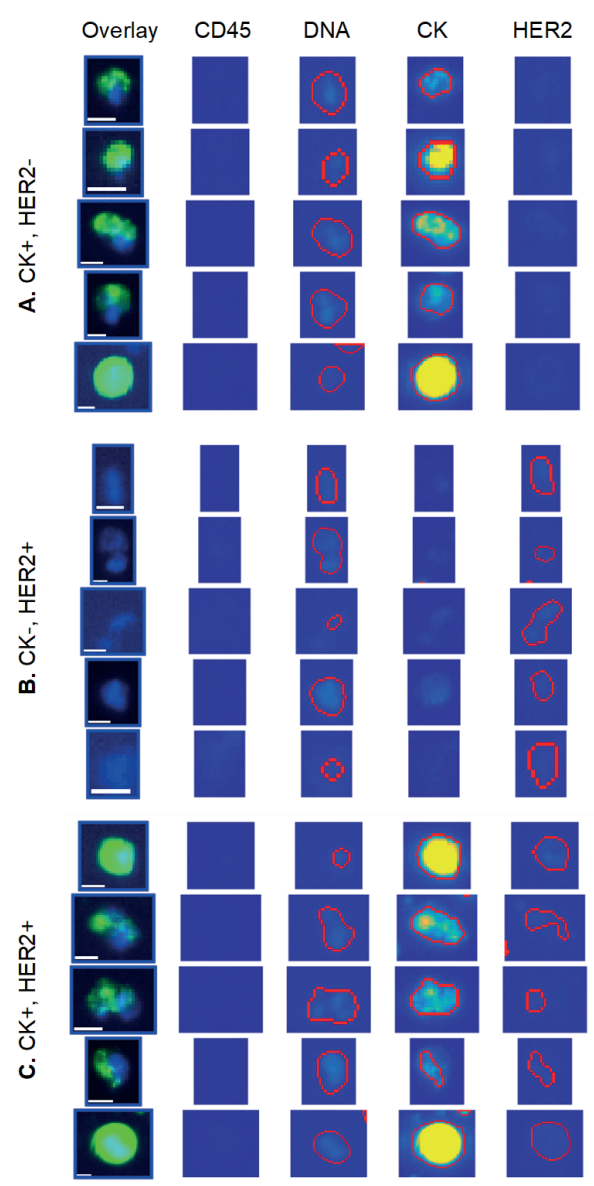

Supplementary Figure S6.1. ACCEPT display of different subclasses of CTCs (left) and tdEVs (right) isolated by the CellSearch system from metastatic breast cancer patients. Examples of CK+HER2(Panel A), CK-HER2+ (Panel B) and CK+HER2+ (Panel C) CTCs and tdEVs. The red contours around the objects in the respective channel indicate the detected and segmented signal by the ACCEPT image analysis algorithm. All objects were isolated from $7.5 \mathrm{~mL}$ of blood using the Cellsearch system. Scale bars indicate $6.4 \mu \mathrm{m}$. 
A

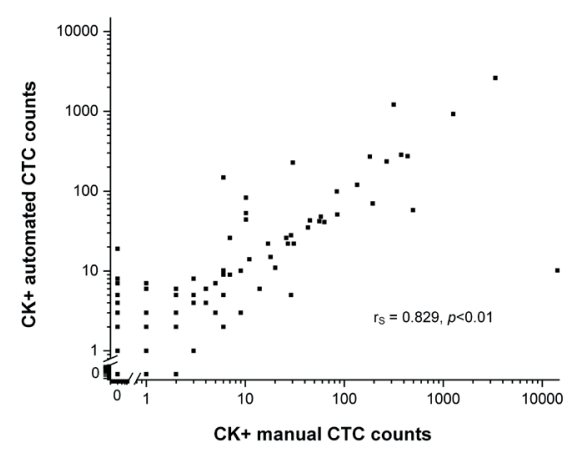

B

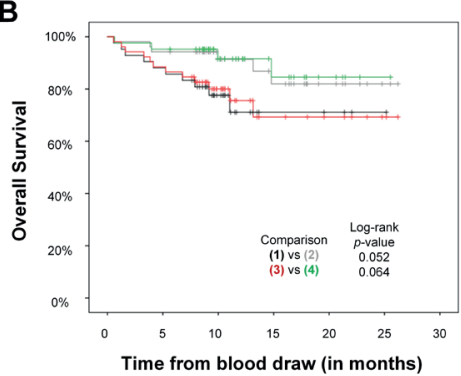

\begin{tabular}{|c|c|c|c|c|c|c|c|}
\hline $5 \mathrm{CK}+\mathrm{mCTCs}$ & 52 & 49 & 31 & 17 & 8 & 2 & 0 \\
\hline $5+\mathrm{CK}+\mathrm{mCTCs}$ & 42 & 37 & 19 & 4 & 3 & 1 & 0 \\
\hline$<5 \mathrm{CK}+\mathrm{aCTCs}$ & 42 & 40 & 24 & 12 & 5 & 1 & 0 \\
\hline $5+\mathrm{CK}+\mathrm{aCTCs}$ & 52 & 46 & 26 & 9 & 6 & 2 & 0 \\
\hline
\end{tabular}

Figure S6.2. Correlation of manual with automated CK+ CTC counts and association with clinical outcome of patients. Scatter plot of CK+ manual CTCs (mCTCs) versus CK+ automated CTCs (aCTCs) showing strong correlation (Panel A). KM plots of OS (Panel B) for patients with $<$ and $\geq 5$ CTCs. The dichotomization of patients was done based on either manual (black and grey lines) or automated (red and green) CTC counts showing equivalent association to OS. 
CTCs
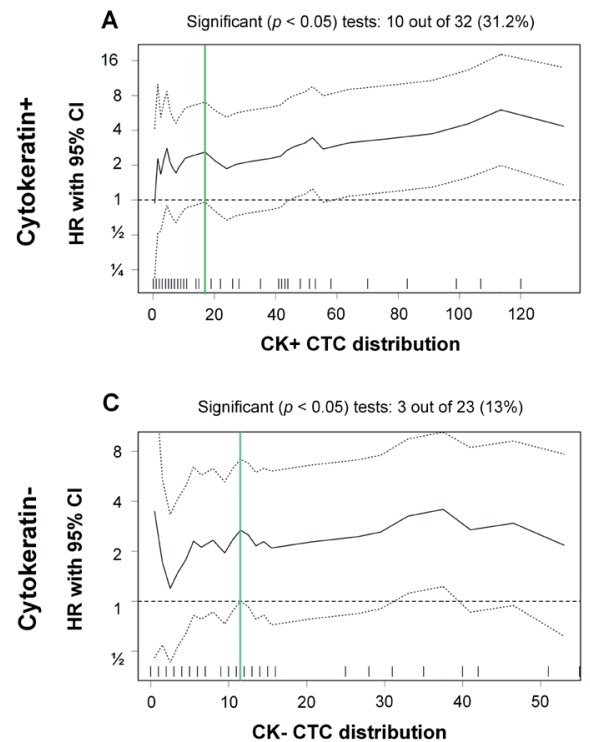

tdEVs

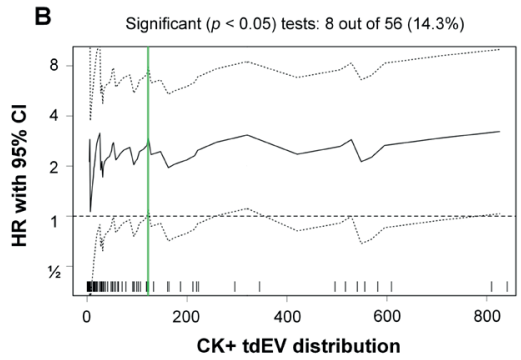

D Significant $(p<0.05)$ tests: 7 out of $23(30.4 \%)$

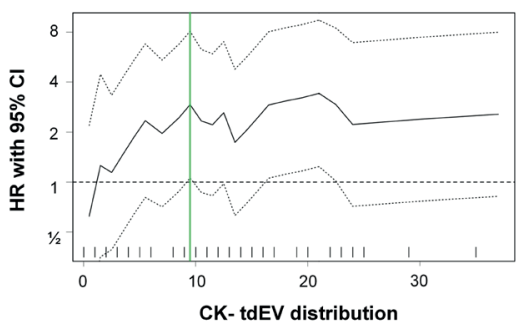

Figure S6.3. Selection of CK+ and CK- CTC and tdEV cut-off values. The HR of OS including $95 \%$ CI was plotted in dependence of the cut-off values for CK+ CTCs (Panel A), CK+ tdEVs (Panel B), CK- CTCs (Panel C) and CK- tdEVs (Panel D). The green vertical lines indicate the minimal cut-off values that result in significant correlation with OS and were chosen. The rug plots at the bottom of Panels A-D correspond to the value distributions of CK+ CTCs, CK+ tdEVs, CK- CTCs and CK- tdEVs respectively. For CK+ CTCs, a larger percentage of cut-off values (31\%, Panel A) could significantly dichotomize patients with a higher and lower risk as compared to CK- CTCs (13\%, Panel C). The opposite was observed for tdEVs with a larger percentage of cut-off values for CK-tdEVs (30\%, Panel D) leading to a significant dichotomization of patients as compared to CK+tdEVs (14\%, Panel B). 
A

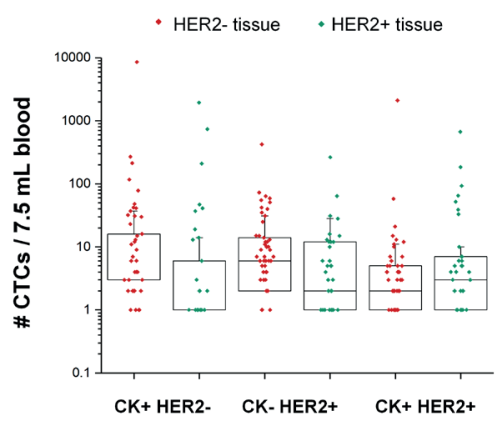

C

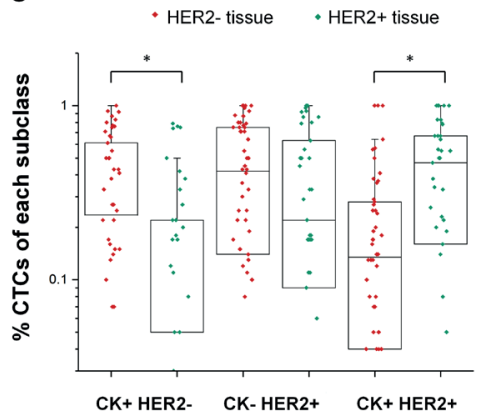

B

tdEVs

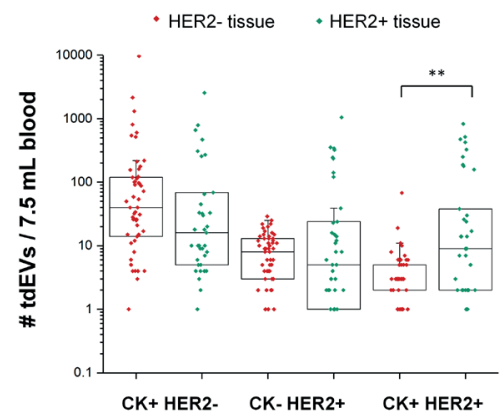

D

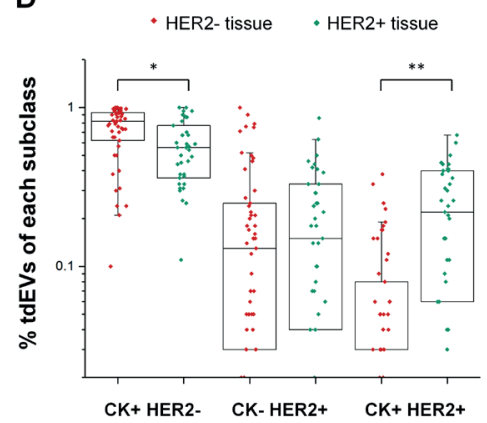

Figure S6.4. Comparison of patients with HER2+ and HER2- tissues in regards to their relative and absolute frequencies of CTCs and tdEVs of different phenotypes. Box plots with data overlap depicting the automated CTC counts (Panel A), automated tdEV counts (Panel B), \% of CTCs (Panel C) and \% of tdEVs (Panel D) of the 3 different immunophenotypes (indicated in the $\mathrm{x}$-axis) in metastatic breast cancer patients split based on the HER2 status of their tissue. Each dot corresponds to the counts of one patient (green dots: patients with HER2+ tissue ( $\mathrm{N}=39$ ), red dots: patients with HER2tissue ( $\mathrm{N}=53$ ), there was no available HER2 status for $\mathrm{N}=6$ patients). Lower and upper bounds of box plots correspond to the $1^{\text {st }}(\mathrm{Q} 1)$ and $3^{\text {rd }}\left(\mathrm{Q}_{3}\right)$ quartile of data, horizontal black lines indicate median vaues. Whiskers indicate $1.5^{*}\left(\mathrm{Q}_{3}-\mathrm{Q} 1\right)$. * indicates significant $(\mathrm{p}<0.05)$ and ** highly significant $(\mathrm{p}<$ 0.001) statistical difference (Mann-Whitney U test). 



\title{
Chapter 7
}

\section{Monitoring responses to therapy with EpCAM+ CTCs and large tdEVs}

Afroditi Nanou and Leon W.M.M. Terstappen

\begin{abstract}
Circulating tumor cell (CTC) and large tumor-derived extracellular vesicle (tdEV) loads are equally associated with poor overall survival (OS) in castration-resistant prostate cancer (CRPC), metastatic breast cancer (MBC) and metastatic colorectal cancer (mCRC). The frequency of tdEVs is an order of magnitude higher than CTCs and as such, tdEVs can potentially represent a more reliable measurement. Here, we compared the presence of CTCs and tdEVs before and after the first cycle of therapy in $190 \mathrm{CRPC}, 179 \mathrm{MBC}$ and $450 \mathrm{mCRC}$ patients to evaluate the effect of therapy on CTCs versus tdEVs. The association of CTCs and tdEVs with OS was similar before the initiation of therapy but after the first cycle of therapy, CTC counts were superior to tdEV counts. Correlation between CTCs and tdEVs was higher before than after the first cycle of therapy. Sorting the patients into those that favorably responded to therapy based on declining CTCs versus those that did not, showed a more profound effect on declines of CTCs than tdEVs. One of the potential explanations is that effective therapy goes along with an increase in tumor-derived apoptotic bodies, which are included in the total tdEV counts. The distinction of different tdEV subclasses using a deep learning approach encourages us to determine the subclasses that rise or decline after the administration of an effective therapy in order to improve the evaluation of therapy responses and the patient treatment monitoring.
\end{abstract}




\subsection{Introduction}

Circulating tumor cells (CTCs) and large tumor-derived extracellular vesicles (tdEVs), detected by the CellSearch system are associated with poor clinical outcome with patients with higher CTC and tdEV loads in their blood having worse survival ${ }^{1-8}$. Several studies have demonstrated the potential of CTCs as a surrogate marker of treatment efficacy ${ }^{9-15}$. A bottleneck of most of these analyses is the scarcity of CTCs and the dichotomization of patients into favorable and unfavorable prognostic groups using a very low static cut-off of 3 or 5 CTCs. Assessment of response can only be determined in patients with unfavorable CTC counts before treatment, whereas conversion of patients to a group with lower/higher risk when CTC counts before and after treatment are close to the CTC cut-off (e.g. from 6 to 4 CTCs or vice versa) is not reliable ${ }^{16}$. Since large tdEVs are present at 20-fold higher frequency than CTCs, they are equally associated with poor outcome of patients as CTCs and they can further stratify patients with favorable CTCs into favorable and unfavorable groups ${ }^{7}$, we explored whether tdEVs could improve the accuracy of assessment of therapy response.

\subsection{Materials and Methods}

\subsubsection{CellSearch images of cancer patients}

Cellsearch image datasets from 190 CRPC, 179 MBC, and 450 mCRC patients from the previously reported IMMC $38^{8}, \mathrm{IMMCO}^{4}$ and CAIRO $2^{1}$ studies were used for this analysis. For CRPC, 190 samples before treatment (baseline, BL) and 166 samples after 2-5 weeks from the initiation of the first cycle of therapy (follow up 1, FU1) were used. For $\mathrm{MBC}, 179 \mathrm{BL}$ samples and $102 \mathrm{FU1}$ samples (3-5 weeks from $\mathrm{BL}$ ) were used. For mCRC, $450 \mathrm{BL}$ samples, $332 \mathrm{FU} 1$ and $317 \mathrm{FU} 2$ samples after the first cycles of therapy (1-2 weeks and 3-4 weeks from BL, respectively) were used.

\subsubsection{Isolation and detection of CTCs and tdEVs}

For CellSearch analysis, $7.5 \mathrm{~mL}$ of blood collected in CellSave tubes (Menarini, Huntingdon Valley, PA, USA) were used. Blood samples of all cancer patients were processed with the CellSearch system within 96 hours from the time of blood draw. The CellSearch system consists of the CellTracks Autoprep ${ }^{\circledR}$ and the CellTracks Analyzer $\mathrm{II}^{\circledR}$. The CellTracks Autoprep is used to immunomagnetically enrich EpCAM+ objects from blood and stain the enriched objects with the nuclear dye DAPI, phycoerythrinconjugated antibodies against the epithelial-specific cytokeratins 8, 18 and 19 (CK$\mathrm{PE}$ ) and an allophycocyanin-conjugated antibody against the leukocyte-specific marker CD45 (CD45-APC) ${ }^{17}$. The enriched labeled objects end up in a cartridge with an optically transparent cover slip. The cartridge is placed in a CellTracks Magnest, a device consisting of two magnets held together by an iron yoke. The shape and 
configuration of both the Magnest and the cartridge allow all enriched objects align in a homogenous distribution on the cover slip following the magnetic field line trajectories of the specific shape and position of the magnets. The CellTracks Analyzer II acquires digital images of $0.65 \times 0.65 \mu \mathrm{m}^{2}$ sized pixels from the whole surface of the cover slip in four different fluorescence channels using a 10×/0.45 NA objective and a charge-coupled device camera. For each cartridge, 144-180 4-layer (DAPI, FITC, PE, APC) tiff images are saved.

\subsubsection{Enumeration of CTCs and tdEVs}

Manual CTC scores were used and tdEVs were enumerated using the open-source ACCEPT software developed in the frames of CTCTrap (www.utwente.nl/en/tnw/ ctctrap/) and CANCER-ID EU (www.cancer-id.eu) programs. Briefly, the digitally stored Cellsearch fluorescence images are processed by ACCEPT to identify objects using multiscale segmentation ${ }^{18}$. Objects are shown in a gallery of images as well as in scatter plots using the various parameters measured for each object and can be categorized configuring linear gates on the measured parameters. The gates used here for tdEV enumeration were the same as previously reported ${ }^{7}$.

\subsubsection{Statistical analysis}

Statistical analysis was performed using SPSS 23.0 (SPSS Inc., Chicago, IL, USA). For each cancer type, the non-parametric two-tailed Spearman's Rho test was performed to evaluate the relation between CTC and tdEV counts before the initiation of a new treatment (BL) and after a cycle of treatment (FU1 or FU2). The non-parametric Wilcoxon Signed Rank test was used to test the equality of distributions for the matched CTC (or tdEV) counts at BL and at FU1 and FU2 for the responders or the patients with progressing disease, for each cancer type. Overall Survival (OS) for each patient was defined as the elapsed time in months between the BL blood draw date and the date of death or the date of last FU. Patients still alive or lost during the follow-up period were censored. The established CTC cut-off values were used in the present analysis (namely 5 for CRPC and MBC and 3 for $\mathrm{mCRC})^{1,4,8}$, whereas the used tdEV cut-off values were the ones that further dichotomize patients with favorable CTC counts (namely 89 for CRCP, 80 for $\mathrm{MBC}$ and 40 for $\mathrm{mCRC})^{7}$. Kaplan Meier OS curves for favorable and unfavorable prognostic groups based on CTC and/or tdEV counts were compared using the non-parametric log-rank test. Cox proportionalhazards regression analysis was used to determine the univariable hazard ratios (HRs) for OS with $95 \%$ confidence intervals (CIs). 


\subsection{Results}

\subsubsection{CTCs and tdEVs versus overall survival before and after the initiation of therapy}

Kaplan-Meier plots for CRPC, MBC and MCRC patients with favorable and unfavorable CTC and tdEV counts at BL and first FU time point(s) are shown in Figure 7.1. The dichotomization of patients becomes more explicit for all three cancer types when it is based on their CTC counts at FU compared to BL.

On the other hand, the association of tdEVs with the clinical outcome of patients before and after the initiation of a new treatment is dependent on the cancer type or/and the treatment. More specifically, in case of CRPC, similar observations to CTCs can be seen with patients with unfavorable tdEVs in their blood at FU having higher risk of experiencing an event than patients with unfavorable counts at just the $\mathrm{BL}$, as reflected also by the estimated Cox HRs (Table 7.1). In case of MBC, patient stratification based on favorable and unfavorable tdEVs is similar for both CTCs and tdEVs at both time points with a tendency of CTCs performing slightly better at FU. The pattern is differing further for patients stratified based on their CTCs or tdEVs in case of mCRC. In particular, BL tdEV counts perform equally well as BL CTC counts; however, at both FU time points the survival curves based on the FU tdEV values tend to converge in contrast to the respective survival curves based on the FU CTC counts that diverge further than the ones at BL (also reflected by the estimated HRs, table 7.1).

Table 7.1. Univariable Cox regression analyses of CTCs and tdEVs at BL and FU time points

\begin{tabular}{|c|c|c|c|c|c|c|}
\hline Cancer type & CTCs & HR (95\% CI) & $\mathrm{p}$-value & tdEVs & HR $(95 \% \mathrm{CI})$ & p-value \\
\hline \multirow{2}{*}{ CRPC } & CTCs $B L \geq 5$ & $2.4(1.7-3.5)$ & $<0.001$ & tdEVs $B L \geq 89$ & $2.8(1.9-4.0)$ & $<0.001$ \\
\hline & CTCs FU1 $1 \geq 5$ & $3.6(2.5-5.2)$ & $<0.001$ & tdEVs FU1 $\geq 89$ & $4.4(3.0-6.5)$ & $<0.001$ \\
\hline \multirow{2}{*}{ MBC } & CTCs $B L \geq 5$ & $2.7(1.8-4.1)$ & $<0.001$ & $\operatorname{tdEVs} \mathrm{BL} \geq 80$ & $2.8(1.9-4.2)$ & $<0.001$ \\
\hline & CTCs FU1 $\geq 5$ & $3.2(1.9-5.4)$ & $<0.001$ & tdEVs $F_{1} \geq 80$ & $2.7(1.6-4.6)$ & $<0.001$ \\
\hline \multirow{3}{*}{ mCRC } & CTCs $\mathrm{BL} \geq 3$ & $2.3(1.8-3.0)$ & $<0.001$ & tdEVs $B L \geq 40$ & $2.6(2.0-3.4)$ & $<0.001$ \\
\hline & CTCs $\mathrm{FU} 1 \geq 3$ & $4.6(2.9-7.2)$ & $<0.001$ & tdEVs FU $1 \geq 40$ & $2.2(1.5-3.1)$ & $<0.001$ \\
\hline & CTCs FU $2 \geq 3$ & $6.7(4.3-11.4)$ & $<0.001$ & tdEVs FU2 $\geq 40$ & $2.1(1.5-3.1)$ & $<0.001$ \\
\hline
\end{tabular}




\section{CTCs}
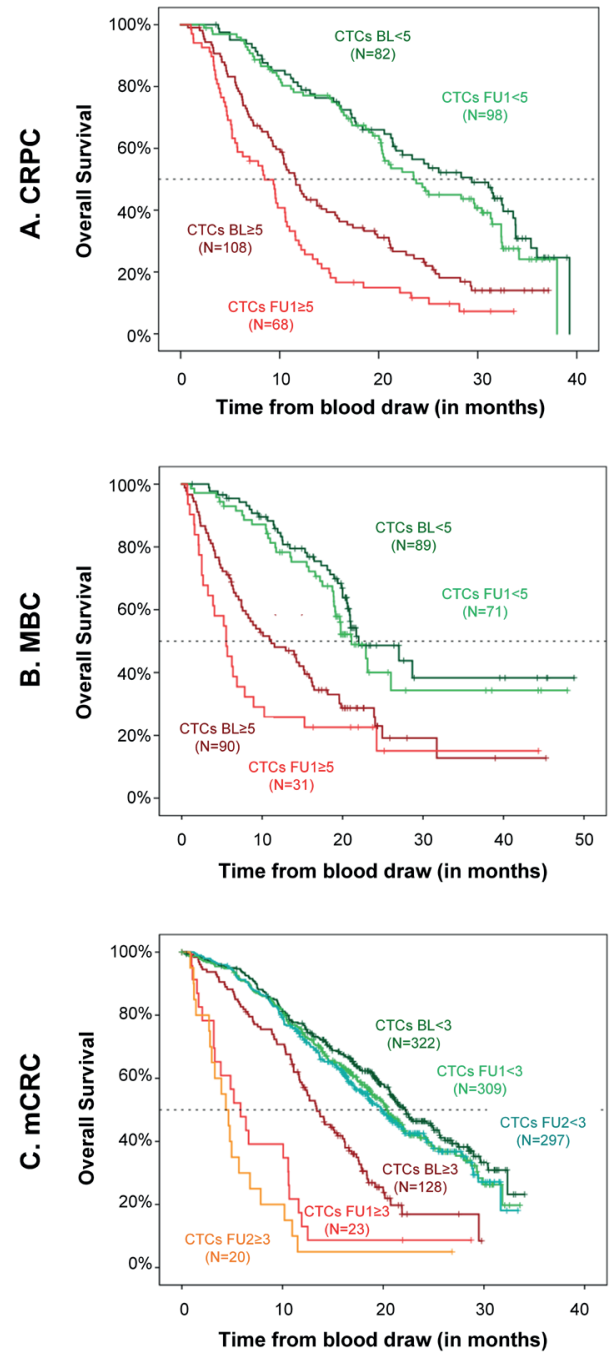

\section{II. tdEVs}
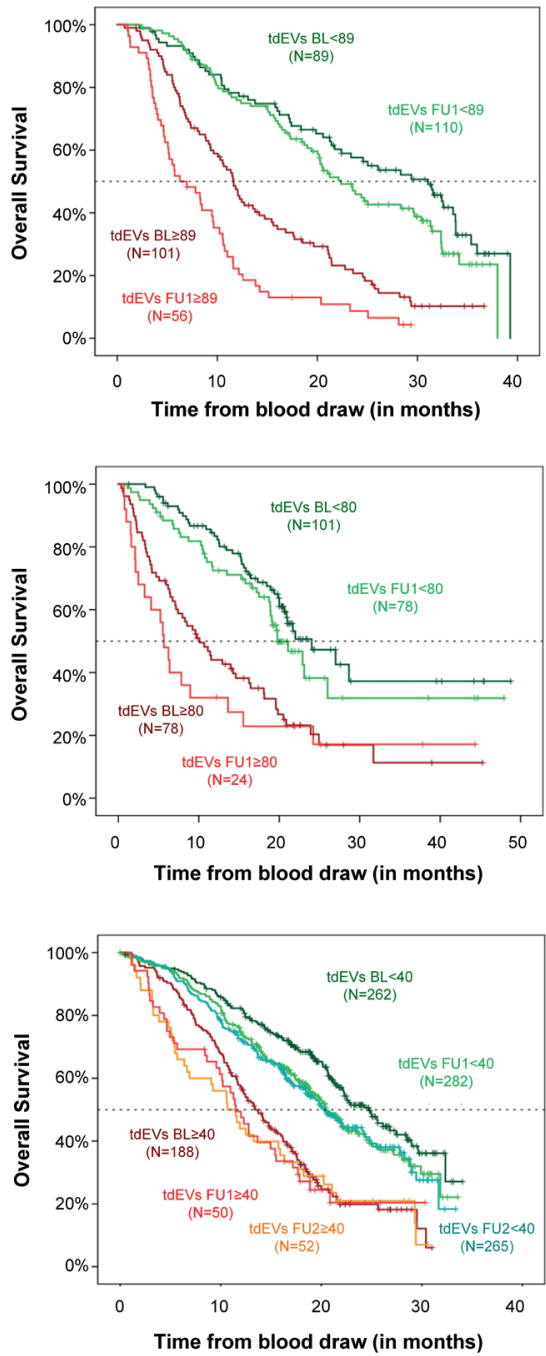

Figure 7.1. I. Kaplan Meier plots of Overall Survival of A. 190 CRPC patients (IMMC38), B. 179 MBC patients (IMMCoI) and C. $450 \mathrm{mCRC}$ patients (CAIRO 2) dichotomized based on their CTC counts (< or $\geq 5$ in case of A. CRPC and B. MBC and $<$ or $\geq 3$ in case of C. $M C R C$ ) before the initiation of a new treatment $(\mathrm{BL})$ and one or two time points after the initiation of a new treatment (FU1, FU2). II. Kaplan Meier plots of Overall Survival of the same patients dichotomized based on their tdEV counts ( $<$ or $\geq 89$ in case of CRPC, 80 in case of MBC and 40 in case of $\mathrm{mCRC}$ ) at BL, FU1 and FU2. The number of patients is reduced at $\mathrm{FU}$ time points. 
To further investigate potential causes of the less significant association of tdEVs with clinical outcome at FU as compared to BL, we assessed the association between CTC and tdEV counts (Figure 7.2) for all cancer types. In CRPC, rank correlation of CTCs and tdEVs was strongly positive at both BL and FU1, as estimated by the Spearman's rank correlation coefficient $r_{s}(0.87 \mathrm{vs} 0.81)$. In $\mathrm{MBC}$, the rank correlation was found moderate at both BL and FU1 (0.71 vs 0.65 ), whereas in mCRC a moderate rank correlation was found at BL which rendered weak at FU1 and FU2 (0.70 vs 0.37 and 0.35 ).

It becomes apparent that the association between CTCs and tdEVs, mainly in case of $\mathrm{mCRC}$, becomes weaker over the course of the treatment. Since CTCs perform better than tdEVs in the FU time point in predicting the clinical outcome of $\mathrm{mCRC}$ patients, we investigated the change in both CTCs and tdEVs from BL to FU for the most interesting subsets of patients (of all three cancer types), namely the treatment responders and the ones with progressing disease. For this analysis, responders were defined as the patients that had converted CTC counts in their blood at FU time point from unfavorable ( $\geq 5$ CTCs in case of CRPC and MBC, $\geq 3$ CTCs in case of $\mathrm{mCRC}$ ) to favorable ( $<5$ CTCs in case of CRPC and MBC, $<3$ CTCs in case of $\mathrm{mCRC}$ ) prognostic group and those that progressed were defined as the ones with converted CTC counts from favorable to unfavorable group or with unfavorable CTC counts in both time points. The number of patients in each group is listed in Table 7.2. The CTC and tdEV counts at both time points are illustrated in Figure 7.3.

Table 7.2. Definition of patients responding to treatment (in green) or with progressing disease (in red) based on their CTC counts at BL and FU1 time points. " 3 in case of mCRC

\begin{tabular}{|c|c|c|c|c|c|c|c|}
\hline \multicolumn{2}{|c|}{ CTC counts } & \multicolumn{6}{|c|}{ Cancer type } \\
\hline $\mathrm{BL}^{*}$ & FU1* & \multicolumn{2}{|c|}{$\begin{array}{c}\text { CRPC } \\
(\mathrm{N}=166)\end{array}$} & \multicolumn{2}{|c|}{$\begin{array}{c}M B C \\
(\mathrm{~N}=102)\end{array}$} & \multicolumn{2}{|c|}{$\begin{array}{c}\text { mCRC } \\
(\mathrm{N}=317)\end{array}$} \\
\hline$<5$ & $<5$ & 68 & & 45 & & 219 & \\
\hline $\begin{array}{l}<5 \\
\geq 5\end{array}$ & $\begin{array}{l}\geq 5 \\
\geq 5\end{array}$ & $\begin{array}{l}5 \\
63\end{array}$ & 68 & $\begin{array}{c}3 \\
28\end{array}$ & 31 & $\begin{array}{c}5 \\
15\end{array}$ & 20 \\
\hline$\geq 5$ & $<5$ & 30 & 30 & 26 & 26 & 78 & 78 \\
\hline
\end{tabular}

Individuals that responded to therapy (based on their CTC conversion to favorable group) had a significant ( $p<0.001$, Wilcoxon Signed Ranks test) decrease of CTC and tdEV counts at FU compared to their respective BL values. However, the mean relative decrease of CTCs was always more profound as compared to the respective decrease of tdEVs, as shown in percentages above each line series. For progressing patients, no significant (Wilcoxon Signal Ranks test) changes were found in CTC and tdEV values at FU time point as compared to BL; although the relative CTC increases were again more pronounced than the respective tdEV increases (apart from the CRPC case, where similar changes were found). 


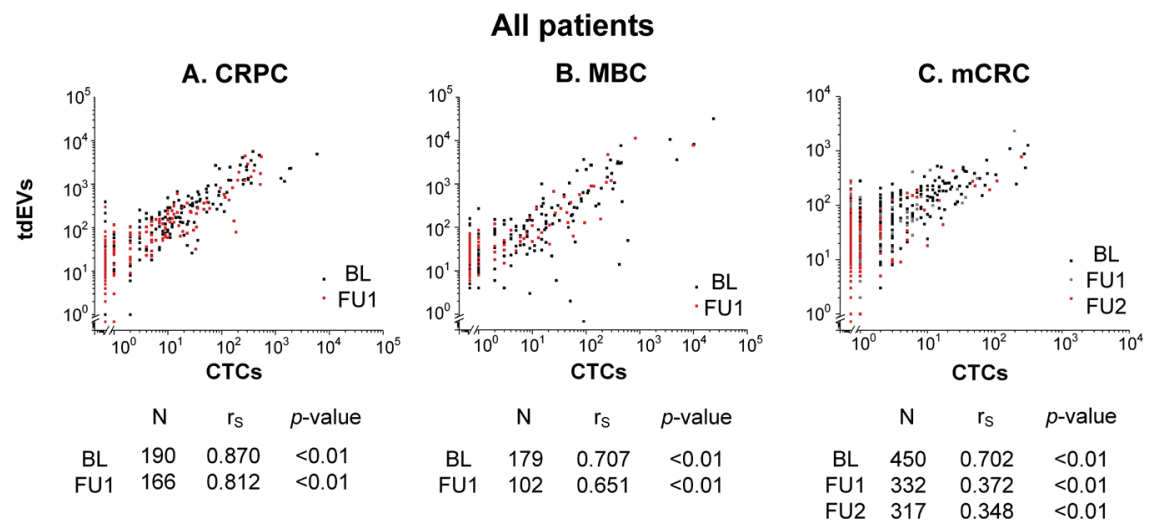

Figure 7.2. Scatter plots of CTCs and tdEVs before and after the initiation of a new treatment. The Spearman's Rho correlation coefficient $\left(r_{s}\right)$ was used to evaluate the strength of relation between CTCs and tdEVs via a monotonic function.
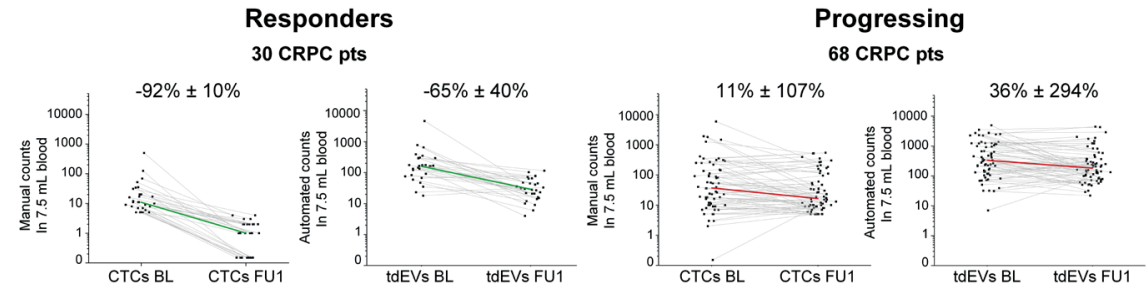

$26 \mathrm{MBC}$ pts

$31 \mathrm{MBC}$ pts
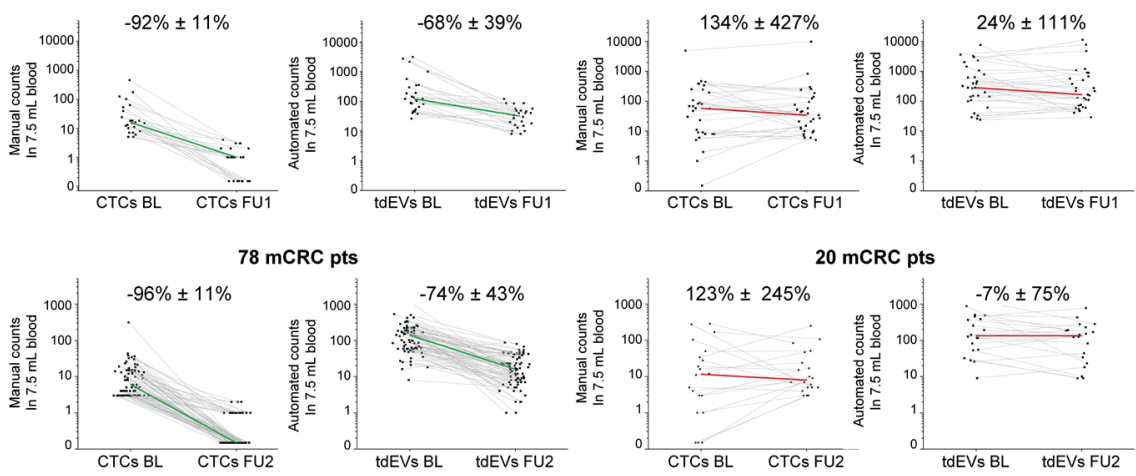

8 mCRC pts

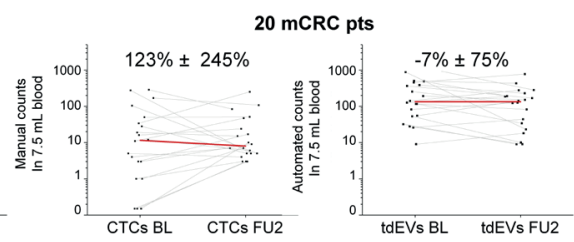

Figure 7.3. CTC and tdEV counts at BL and FU1 or FU2 of treatment responders (left) and individuals with progressing disease (right). The green lines connect the BL and FU median values of CTCs or tdEVs for responders, whereas the red lines connect the median BL and FU median values of CTCs or tdEVs for individuals with progressing disease. The percentages on top of each graph corresponds to the mean relative change of CTC or tdEV counts: (counts FU -counts BL)/counts BL \pm standard deviation. 
A reasonable explanation regarding the lesser relative decreases of tdEVs compared to CTCs in case of responders, is the pooled tdEV subpopulations that fall in our definition of tdEVs isolated with the CellSearch system (namely large EpCAM+, $\mathrm{CK}+, \mathrm{CD} 45^{-}, \mathrm{DAPI}-$ particles). In that definition, both oncosomes and tumor-derived apoptotic bodies have similar characteristics in terms of their membrane proteome (EpCAM) and size. Whereas before the initiation of treatment, active secretion of oncosomes is expected to indicate the metastatic potential of the tumor in a similar way as CTCs, at FU, an effect of the treatment is anticipated in the release of EVs from the tumor. A successful anti-cancer treatment would result in the apoptosis/ destruction of tumor cells and consequently, the passive release of apoptotic bodies in the blood circulation, whereas the active release of oncosomes should be reduced in a similar way as viable CTCs. Likewise, one would expect a relative large portion of apoptotic CTCs as an effect of therapy. A discrimination between apoptotic and viable CTCs should improve the ability to gauge the response to therapy. However, that hypothesis is difficult to be tested since the individuals that responded to treatments were by definition the ones with converted CTC counts from unfavorable to favorable prognostic groups with very few CTCs ( $<3$ in case of $\mathrm{mCRC}$ or $<5$ in case of CRPC and $\mathrm{MBC}$ in $7.5 \mathrm{~mL}$ of blood). In particular, $11 / 30$ (37\%) of CRPC, $10 / 26$ (39\%) of MBC and $60 / 78$ (77\%) of $\mathrm{mCRC}$ responders had no detectable CTCs at FU time points (FU1 in case of CRPC and MBC and FU2 in case of $\mathrm{MCRC}$ ).

Following the same rationale, the worse association of tdEVs with the clinical benefit of patients at FU is logical and points out the necessity of cautious interpretation of increases/ decreases of these large tdEVs over the course of a treatment, mainly in case of mCRC. A marker that could discriminate the subclasses of tdEVs into tumor-derived apoptotic bodies and actively secreted oncosomes would provide a solution to such a problem.

Alternatively, artificial intelligence could detect differences that cannot be recognized by the human eye or a very generic automated gate. Application of a deep learning algorithm on a large dataset of CellSearch images of $\mathrm{MBC}$ patients already demonstrated superiority in the classification of CTCs of MBC patients compared to the manual CTC scoring from trained operators ${ }^{19}$. Importantly, at least 5 different tdEV subpopulations were identified by the network (Figure 7.4), all of which ending in the same pool of objects applying our ACCEPT tdEV gate (as depicted in light blue color) ${ }^{19}$.

Our next step will be to investigate the association of each tdEV subclass identified by deep learning with the clinical outcome of cancer patients. It is possible that some of these subclasses (apoptotic bodies of tumor origin or EVs of healthy epithelial cell origin) are not associated to the overall survival of patients, whereas others (oncosomes or large tumor-derived microvesicles) predict much better the OS of patients than what we report in the present dissertation, where the different subpopulations were neglected. It is worth exploring the effect of different treatments (cytotoxic chemo-, 
hormone, molecular and immuno- therapies) on the stimulation or hindrance of the secretion of each of these $t d E V$ subclasses. Re-analysis of existing data sets at several FU time points after the course of different treatments together with the known response/ clinical outcome of the patients could lead to a deeper understanding of the underlying drug mechanisms as well as the developed resistance mechanisms of the tumor opening the pathway for the development of alternative and more efficient treatments.

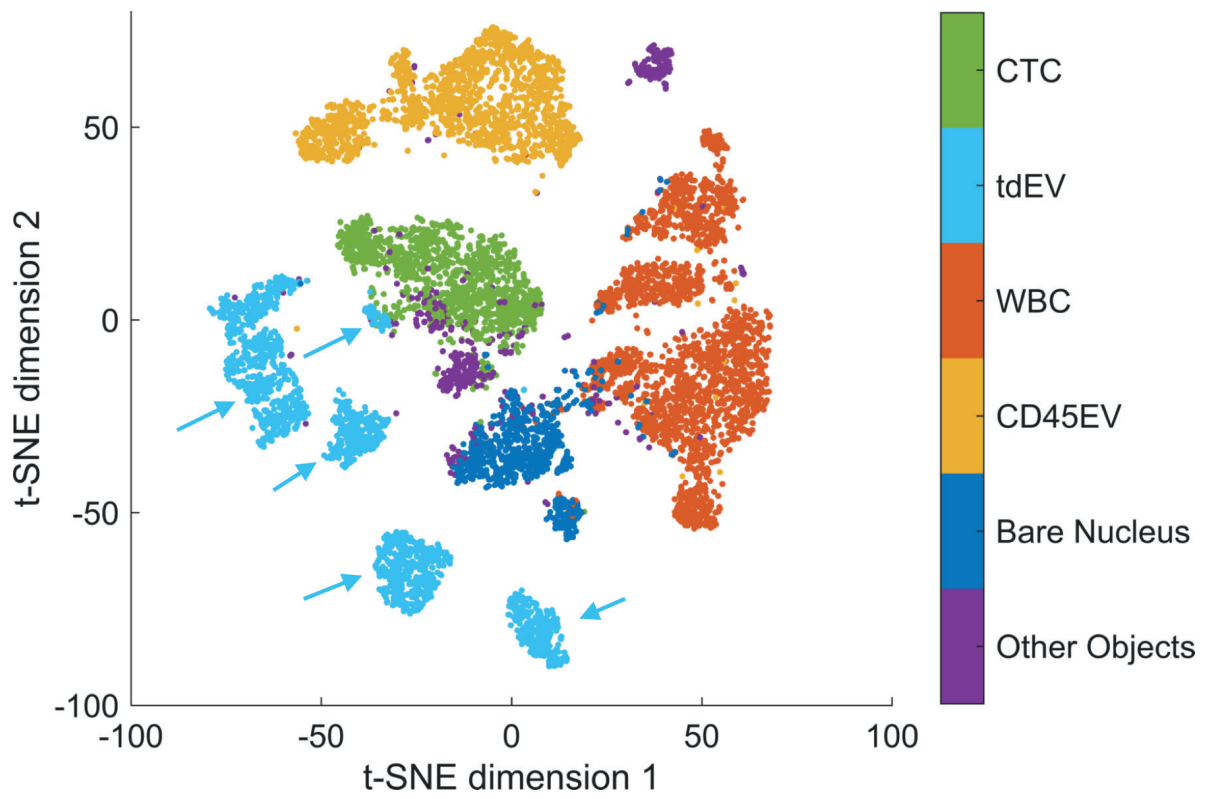

Figure 7.4. Classification and encoded representation of the 6-class data set in form of a twodimensional t-SNE map. Data points are colored based on their class label, as defined by the operator ${ }^{19}$. 


\section{References}

1. Cohen SJ, Punt CJA, Iannotti N, Saidman BH, Sabbath KD, Gabrail NY et al. Relationship of circulating tumor cells to tumor response, progression-free survival, and overall survival in patients with metastatic colorectal cancer. Journal of Clinical Oncology 2008; 26(19): 3213-3221; doi 10.1200/Jc0.2007.15.8923.

2. Scher HI, Heller G, Molina A, Attard G, Danila DC, Jia Xet al. Circulating tumor cell biomarker panel as an individual-level surrogate for survival in metastatic castration-resistant prostate cancer. J Clin Oncol 2015; 33(12): 1348-1355; doi 10.1200/JCO.2014.55.3487.

3. Heller G, Fizazi K, McCormack R, Molina A, MacLean D, Webb IJ et al. The Added Value of Circulating Tumor Cell Enumeration to Standard Markers in Assessing Prognosis in a Metastatic Castration-Resistant Prostate Cancer Population. Clinical Cancer Research 2017; 23(8): 1967-1973; doi 10.1158/1078-0432.Ccr-16-1224.

4. Cristofanilli M, Budd GT, Ellis MJ, Stopeck A, Matera J, Miller MC et al. Circulating tumor cells, disease progression, and survival in metastatic breast cancer. New Engl J Med 2004; 351(8): 781-791; doi DOI 10.1056/NEJMoa040766.

5. Coumans FAW, Doggen CJM, Attard G, de Bono JS, Terstappen LWMM. All circulating EpCAM $+\mathrm{CK}+\mathrm{CD} 45$-objects predict overall survival in castration-resistant prostate cancer. Ann Oncol 2010; 21(9): 1851-1857; doi 10.1093/annonc/mdq030.

6. Nanou A, Coumans FAW, van Dalum G, Zeune LL, Dolling D, Onstenk W et al. Circulating tumor cells, tumor-derived extracellular vesicles and plasma cytokeratins in castrationresistant prostate cancer patients. Oncotarget 2018; 9(27): 19283-19293; doi 10.18632/ oncotarget.25019.

7. Nanou A, Miller MC, Zeune LL, de Wit S, Punt CJA, Groen HJM et al. Tumour-derived extracellular vesicles in blood of metastatic cancer patients associate with overall survival. $\mathrm{Br}$ J Cancer 2020; doi 10.1038/s41416-019-0726-9.

8. Scher HI, Jia XY, de Bono JS, Fleisher M, Pienta KJ, Raghavan D et al. Circulating tumour cells as prognostic markers in progressive, castration-resistant prostate cancer: a reanalysis of IMMC38 trial data. Lancet Oncol 2009; 10(3): 233-239; doi 10.1016/S1470-2045(08)70340-1.

9. Vogelzang NJ, Fizazi K, Burke JM, De Wit R, Bellmunt J, Hutson TE et al. Circulating Tumor Cells in a Phase 3 Study of Docetaxel and Prednisone with or without Lenalidomide in Metastatic Castration-resistant Prostate Cancer. Eur Urol 2017; 71(2): 168-171; doi 10.1016/j. eururo.2016.07.051.

10. Lorente D, Olmos D, Mateo J, Dolling D, Bianchini D, Seed G et al. Circulating Tumor Cell Increase as a Biomarker of Disease Progression in Metastatic Castration-Resistant Prostate Cancer Patients with Low Baseline CTC Counts. Ann Oncol 2018; doi 10.1093/annonc/mdy172.

11. Lorente D, Olmos D, Mateo J, Bianchini D, Seed G, Fleisher M et al. Decline in Circulating Tumor Cell Count and Treatment Outcome in Advanced Prostate Cancer. European Urology 2016; 70(6): 985-992; doi 10.1016/j.eururo.2016.05.023. 
12. Heller G, McCormack R, Kheoh T, Molina A, Smith MR, Dreicer R et al. Circulating Tumor Cell Number as a Response Measure of Prolonged Survival for Metastatic Castration-Resistant Prostate Cancer: A Comparison With Prostate-Specific Antigen Across Five Randomized Phase III Clinical Trials. Journal of Clinical Oncology 2018; 36(6): 572-+; doi 10.1200/Jco.2017.75.2998.

13. de Bono JS, Scher HI, Montgomery RB, Parker C, Miller MC, Tissing H et al. Circulating Tumor Cells Predict Survival Benefit from Treatment in Metastatic Castration-Resistant Prostate Cancer. Clinical Cancer Research 2008; 14(19): 6302-6309; doi 10.1158/1078-0432.Ccr08-0872.

14. De Laere B, Oeyen S, Van Oyen P, Ghysel C, Ampe J, Ost P et al. Circulating tumor cells and survival in abiraterone- and enzalutamide-treated patients with castration-resistant prostate cancer. Prostate 2018; 78(6): 435-445; doi 10.1002/pros.23488.

15. Goldkorn A, Ely B, Quinn DI, Tangen CM, Fink LM, Xu T et al. Circulating tumor cell counts are prognostic of overall survival in SWOG SO421: a phase III trial of docetaxel with or without atrasentan for metastatic castration-resistant prostate cancer. J Clin Oncol 2014; 32(11): 11361142; doi 10.1200/JCO.2013.51.7417.

16. Tibbe AG, Miller MC, Terstappen LW. Statistical considerations for enumeration of circulating tumor cells. Cytometry A 2007; 71(3): 154-162; doi 10.1002/cyto.a.20369.

17. Allard WJ, Matera J, Miller MC, Repollet M, Connelly MC, Rao C et al. Tumor cells circulate in the peripheral blood of all major carcinomas but not in healthy subjects or patients with nonmalignant diseases. Clinical Cancer Research 2004; 10(20): 6897-6904; doi Doi 10.1158/10780432.Ccr-04-0378.

18. Zeune L, van Dalum G, Terstappen L, van Gils S, Brune C. Multiscale Segmentation via Bregman Distances and Nonlinear Spectral Analysis. Siam Journal on Imaging Sciences (Article) 2017; 10(1): 111-146; doi 10.1137/16M1074503.

19. Zeune LLB, Y.; van Dalum, G.; Nanou, A.; de Wit, S.; Andree, K.; Swennenhuis, S.; van Gils , S.; Terstappen, L.W.M.M.; Brune, C. Deep learning of Circulating Tumour Cells. Nature Machine Intelligence (Research Article) 2020. 



\title{
Chapter 8
}

\section{Endothelium- derived extracellular vesicles associate with poor prognosis in metastatic colorectal cancer}

\author{
Afroditi Nanou, Linda Mol, Miriam Koopman, Cornelis J.A. Punt,
}

Leon W.M.M. Terstappen

Submitted for publication.

\begin{abstract}
Elevated tumor-derived Extracellular Vesicle (tdEV) and Circulating Tumor Cell (CTC) load in patients with metastatic cancer is associated with poor clinical outcome. In this study, we investigated whether endothelium-derived extracellular vesicles (edEVs) can be detected in the blood of metastatic colorectal cancer (mCRC) patients and whether they associate with prognosis. The open-source ACCEPT software was used to enumerate edEVs, tdEVs and other objects from digitally stored CellSearch ${ }^{\circledR}$ images obtained after CTC and Circulating Endothelial Cell (CEC) enrichment from blood of 395 mCRC patients before initiation of a new therapy. ACCEPT classified objects, CTC and CEC counts and patient information were used in univariable and multivariable analyses to determine their relation with Progression-Free Survival (PFS) and Overall Survival (OS). edEVs are present in blood of mCRC patients at 5- to 10-fold higher frequencies than CECs. A significantly shorter PFS and OS was observed for patients with $\geq 3$ CTCs $/ 7.5 \mathrm{~mL}, \geq 40 \mathrm{tdEVs} / 7.5 \mathrm{~mL}$ and $\geq 287 \mathrm{edEVs} / 4.0 \mathrm{~mL}$. The Hazard Ratio HR (95\% CI) of PFS for increased CTCs, tdEVs and edEVs was 1.4 (1.1-1.9), 2.0 (1.5-2.6) and 1.7 (1.2-2.5), respectively. Similarly, the HR of OS for increased CTCs, tdEVs and edEVs was 2.2 (1.7-3.0), $2.7(2.0-3.5)$ and 2.1 (1.5-2.8), respectively. There was no cut-off value for CECs leading to a dichotomization of patients with a significant HR. Both tdEVs and edEVs remained significant predictors of OS in the final multivariable model.
\end{abstract}




\subsection{Introduction}

The necessity of non-invasive, disease-specific and reliable biomarkers in different cancers is becoming urgent for the administration of targeted therapies and the evaluation of their efficacy in a timely fashion. In approximately $30 \%$ of advanced colorectal cancer patients, 3 or more Circulating Tumor Cells (CTCs) in $7.5 \mathrm{ml}$ of blood are detected and these patients have a significantly shorter progression-free (PFS) and overall survival (OS) ${ }^{1,2}$. Therapy monitoring through increases or decreases in the number of CTCs is therefore, only meaningful in these $30 \%$ of patients. Recently, we have shown that tumor-derived extracellular vesicles (tdEVs) are co-isolated with the CTCs and can be automatically enumerated in the fluorescent images ${ }^{3}$. These tdEVs are present in approximately 20 -fold higher frequencies as compared to CTCs in patients with advanced prostate, breast, nonsmall cell lung and colorectal cancer and their presence is also strongly associated with poor clinical outcome ${ }^{3-5}$. The presence of Circulating Endothelial Cells (CECs) in blood of cancer patients has also been investigated and has been found in higher frequencies in blood of patients with advanced cancer as compared to healthy donors ${ }^{6}$. The release of a portion of the detected endothelial cells can be attributed to their detachment from the blood vessel wall during the blood draw. As this varies between collected blood samples, it has a significant influence on the actual CEC counts and their reliability to follow disease processes ${ }^{6}$. We postulated that if endothelial cells produce extracellular vesicles in a similar way as tumor cells, we might be able to detect them and their count will most likely be more reliable since their formation will be independent of the blood draw and the damage of the vasculature. We used the open-source ACCEPT software (http://github. com/LeonieZ/ACCEPT) to enumerate tdEVs and explore the presence of endotheliumderived extracellular vesicles (edEVs). For this study, we used a digitally stored set of fluorescent images generated from blood samples of advanced colorectal cancer patients receiving first line chemotherapy and targeted agents before the initiation of a new therapy. The blood samples were processed with the CellSearch CTC kit for CTC and tdEV enumeration and the CellSearch Endothelial Cell kit for CEC and edEV enumeration. The CTC and CEC data have been reported earlier ${ }^{2,7}$.

\subsection{Materials and Methods}

\subsubsection{Patients}

All patients included in this study participated in the CAIRO 2 trial (ClinicalTrials.gov Identifier: NCT00208546) of the Dutch Colorectal Cancer Group ${ }^{8}$. The institutional review boards at each center approved the study protocol, and all patients provided written informed consent. Patients were randomly assigned to receive first-line treatment with capecitabine, oxaliplatin, and bevacizumab or the same schedule with the addition of weekly cetuximab. Tumor response was assessed every 9 weeks 
using computed tomography (CT) imaging and evaluated according to the Response Evaluation Criteria in Solid Tumors (RECIST) ${ }^{9}$. Details of the study have been reported previously ${ }^{8,10}$. From this multicenter phase III trial data, CTCs were enumerated before initiation of therapy in 467 of the 755 advanced colorectal cancer patients and CECs in 473 of the 755 patients using the CellSearch system and results have been reported earlier ${ }^{2,7}$. Fluorescence images of samples processed with the CTC and CEC kits were stored on DVDs and transferred to hard disks in 450 of the 457 CTC samples and 395 of the 473 CEC samples. We re-analyzed the stored images of 395 patients for both CTC and CEC samples using the Automated CTC Classification, Enumeration and Phenotyping (ACCEPT) open-source imaging program to identify leukocytes, nucleated cells and subpopulations of Extracellular Vesicles (EVs) co-isolated with both CTC and CEC kits.

\subsubsection{Isolation of CTCs, tdEVs, CECs and edEVs}

The CellSearch CTC assay (Menarini, Huntingdon Valley, PA, USA) was used to immunomagnetically isolate CTCs and tdEVs based on their epithelial cell adhesion molecule (EpCAM) expression from $7.5 \mathrm{~mL}$ of blood collected in CellSave (Menarini, Huntingdon Valley, PA, USA) tubes. The positively enriched objects were stained with the nuclear dye 4',6-diamidino-2-phenylindole (DAPI), allophycocyanin-conjugated monoclonal antibodies against the leukocyte-specific cluster of differentiation 45 (CD45-APC) and phycoerythrin-conjugated monoclonal antibodies against cytokeratins 8,18 and 19 (CK-PE). The suspension was placed in a cartridge contained within a Magnest as previously described ${ }^{11}$. The CellSearch Endothelial Cell assay (Menarini) was used to immunomagnetically enrich for CECs and edEVs expressing CD146 from $4.0 \mathrm{~mL}$ of CellSave blood. The positively enriched objects were stained with with the nuclear dye DAPI and the fluorescently labeled monoclonal antibodies against CD45 (CD45-APC) and endoglin (CD105-PE). Image acquisition of both EpCAM and CD146 enriched and stained objects within cartridges was performed on the CellTracks Analyzer II, a semi-automated fluorescence microscope equipped with computer controlled X, Y, Z stages, a 10x objective with 0.45 numerical aperture, a Mercury Arc lamp, a 12-bit charge-coupled device (CCD) camera and filter cubes for DAPI, PE, APC and fluorescein isothiocyanate (FITC). Typically, 175 images per fluorescence channel are acquired to cover the surface of the whole cartridge ${ }^{11}$.

\subsubsection{Enumeration of tdEVs, edEVs, ldEVs and nucleated cells by ACCEPT}

For this study, the original CTC and CEC scores (manual counts) were used. To enumerate tdEVs, edEVs, leukocyte-derived extracellular vesicles (ldEVs), leukocytes and nucleated cells the digitally stored fluorescence image files were re-analyzed with the open-source ACCEPT software (http://github.com/LeonieZ/ACCEPT) using the "Full Detection" function. For the samples processed with the CEC kit, CD105+ 
leukocytes and CD105+ ldEVs were also identified and enumerated. The gates for each of the abovementioned populations in the samples processed with the CTC and CEC kits are provided in the Supplemental Table S8.1.

\subsubsection{Statistical analysis}

Statistical analysis was performed using the IBM SPSS ${ }^{\circledR}$ platform for Windows, version 23.0 (SPSS Inc., Chicago, IL, USA) and MedCalc ${ }^{\circledR}$ for Windows, version 18.0 (MedCalc Software, Ostend, Belgium). The primary objective was to assess the prognostic value of tdEVs and edEVs in mCRC patients. Eligible patients were assessable for these analyses if both a CTC and CEC sample was available prior to the start of a therapy. Patients were prospectively divided into patients with favorable and unfavorable CTC counts ( $<3 \& \geq 3$, respectively). For tdEVs, the value of 40 was used as a cut-off based on our previous results supporting the additional dichotomization of patients with favorable CTC counts using that value ${ }^{5}$. Overall survival (OS) was defined as the elapsed time in months between the baseline blood draw date and the date of death or last follow-up. Progression-free survival (PFS) was defined as the elapsed time in weeks between the baseline blood draw date and the date of progression or death, whichever occurred first. Patients alive at the end of the study or lost during the follow-up were censored. Median PFS and OS were defined by Kaplan Meier survival curves. Kaplan Meier survival curves of favorable and unfavorable groups were compared using the non-parametric log-rank test.

The open-source web application Cutoff Finder (http://molpath.charite.de/cutoff/) was used to calculate the HRs for PFS and OS, including $95 \%$ confidence intervals (CI), over a wide range of cut-off values for CECs and edEVs of the 395 patients before the initiation of a new treatment. The optimal cut-off value of edEVs was defined as the points with the most significant split (min p-value, log-rank). Cutoff Finder uses the $\mathrm{R}$ code to provide optimization and visualization tools for cut-off determination ${ }^{12}$.

Univariable Cox proportional hazards regression analysis was performed to determine the HR for PFS and OS with 95\% CI for each continuous and categorical variable. Continuous complete blood count ( $\mathrm{CBC}$ )-based parameters, counts of CECs, CTCs, leukocytes, nucleated events and EVs isolated with the CEC and CTC kits were log transformed to improve the model fit. In case of the isolated events with the CTC and CEC kits, a constant value of 0.1 was added to deal with o values. A final multivariable Cox model was fit including the significant variables from the univariable Cox proportional hazards regression analysis. Due to correlation between some of the included variables, the final model was selected using backward stepwise elimination ( $p_{\text {in }}=0.05$ and $p_{\text {out }}=0.10$ ). 
A two-tailed Spearman's Rho test was used to assess the relation between CBC-based parameters and different classes of objects isolated with the Cellsearch using the CTC and CEC kits. The non-parametric Wilcoxon Signed Ranks test was used to compare the distributions of the matched leukocytes, ldEVs and nucleated events isolated with the different kits. Receiver Operating Characteristic (ROC) Curves were used to assess the performance of a test using solely edEVs, CTCs, tdEVs or a combination of edEVs with either CTCs or tdEVs. The classification variable was the shorter patient survival compared to the median OS of the patient cohort used. The Areas Under the Curve (AUC) were compared using the non-parametric DeLong approach ${ }^{13}$.

\subsection{Results}

\subsubsection{Identification of tdEVs, edEVs, ldEVs, leukocytes and nucleated cells by ACCEPT}

Digitally stored fluorescent images, which were originated from the CellTracks Anayzer II, were analyzed by ACCEPT to identify tdEVs, edEVs, ldEVs, leukocytes and nucleated cells using the gates provided in Supplemental Table S8.1. Figure 8.1 shows an example of a scatter plot for tdEVs (Panel A), edEVs (Panel B), ldEVs (Panel C) and leukocytes (Panel D). The ldEVs and leukocytes presented in this example were coisolated using the CTC kit. For tdEVs, the CK-PE mean intensity is plotted against the DAPI mean intensity; for edEVs, the CD105-PE mean intensity is plotted against the DAPI mean intensity; for ldEVs and leukocytes the CD45-APC mean intensity is plotted against the DAPI mean intensity. Next to each scatter plot, four images of objects falling in each gate are shown and the position of each image in the scatter plot is indicated with numbers 1 through 4 . Nucleated cells, of which the lineage of origin could not be determined due to lack of CD45 and CK / CD105 expression fall on the $\mathrm{Y}$-axis of panel $\mathrm{D}$.

\subsubsection{Frequencies of CTCs, tdEVs, CECs and edEVs in colorectal cancer patients before the initiation of a new therapy}

CTCs, tdEVs, CECs and edEVs were enumerated in $395 \mathrm{mCRC}$ patients after immunomagnetic enrichment using the CellSearch system. The results are summarized in Figure 8.2. CTCs ranged from 0-312 (median: 0 , interquartile range (IQR): 0-3), tdEVs from 0-1268 (median: 30, IQR: 14-85), CECs from 0-1250 (median: 27, IQR: 14-59) and edEVs from 1-1504 (median: 139, IQR: 82-238). 
A. Tumor-derived Extracellular Vesicles (tdEVs)

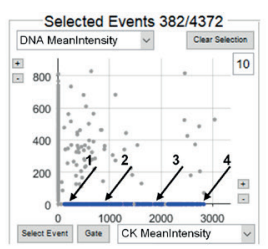

C. Leukocyte-derived EVs (IdEVs)

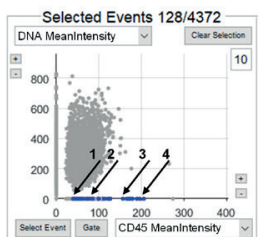

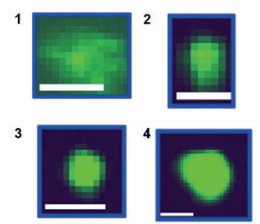
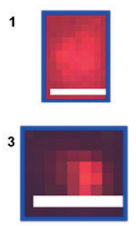
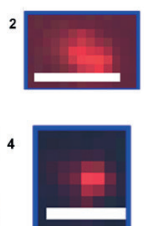

B. Endothelium-derived EVs (edEVs)
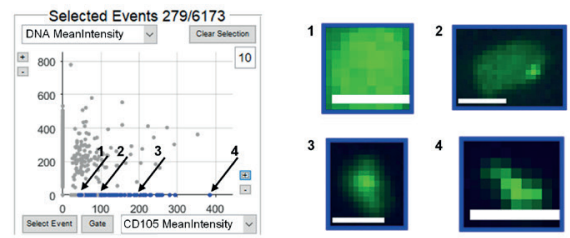

D. Leukocytes
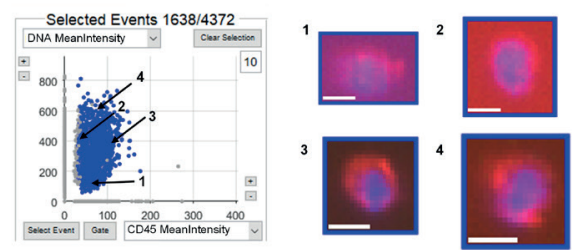

Figure 8.1. Different classes of objects, isolated with the CellSearch system, as identified by the ACCEPT software. Examples of tdEVs (Panel A), edEVs (Panel B), ldEVs (Panel C) and leukocytes (Panel D) found in single cartridges. The dots corresponding to the objects that fall within the defined gates are depicted in blue in the respective scatter plots, whereas all other objects are depicted in grey. For each class, examples of 4 objects with different fluorescence intensities (1-4) are shown. CK (Panel A) and CDio5 (Panel B) are depicted in green, CD45 in red and DAPI in blue (Panels C and D). Scale bars indicate $6.4 \mu \mathrm{m}$.

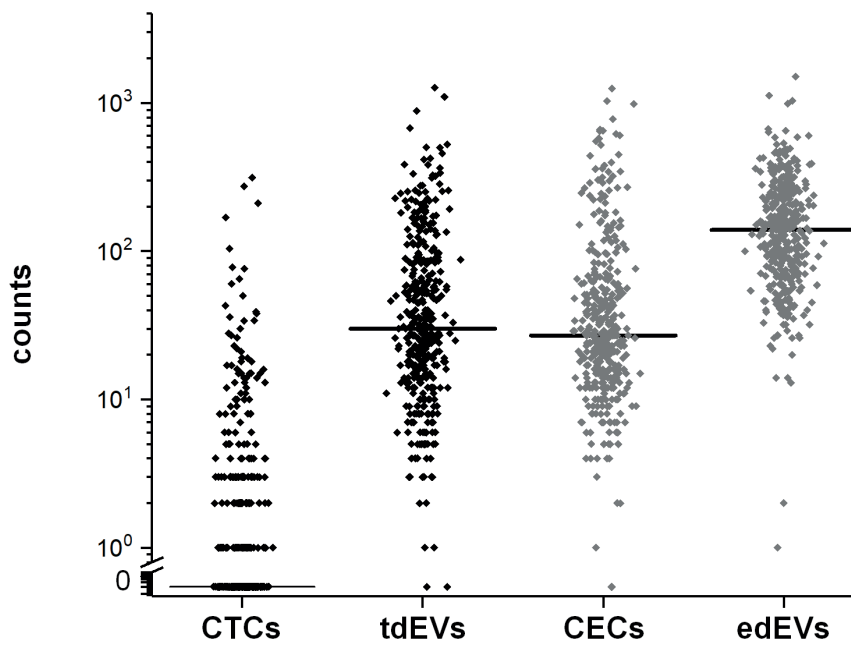

in $7.5 \mathrm{~mL}$ of whole blood

in $4.0 \mathrm{~mL}$ of whole blood

Figure 8.2. Frequencies of Cellsearch isolated CTCs and tdEVs from $7.5 \mathrm{~mL}$ of blood and CECs and edEVs from $4.0 \mathrm{~mL}$ of blood of $395 \mathrm{mCRC}$ patients before the initiation of a new therapy. The horizontal lines correspond to median values. 
In addition to tdEVs and edEVs identified in the image sets generated after processing the blood samples with the CTC and CEC kits nucleated cells of unknown cell lineage of origin, CD45 expressing leukocytes and ldEVs were enumerated. In case of samples processed with the CEC kit, a portion of isolated leukocytes co-expressed CD45 and $\mathrm{CD} 105$ and were most likely monocytes in their differentiation transition to macrophages ${ }^{14,15}$. To facilitate the comparison of the frequencies of the co-isolated events between the two different kits used, all counts were normalized to the initial blood volume processed $(7.5 \mathrm{~mL}$ for samples processed with CTC kit and $4.0 \mathrm{~mL}$ for samples processed with the CEC kit) and the results are shown in Supplemental Figure S8.1. Noteworthy is the observation that in contrast to tdEVs and edEVs that appear in relatively higher frequencies than CTCs and CECs respectively, the number of ldEVs is lower than leukocytes in samples processed with either the CTC or CEC kits. The number of leukocytes and nucleated events isolated with the CEC kit are significantly higher as compared to the number of leukocytes and nucleated events isolated with the CTC kit ( $p<0.01$, Wilcoxon Signed Ranks test). In case of the leukocytes, this observation can be attributed to the expression of CD146 (MCAM) on a T-lymphocyte subset which is enriched with the CD146 ferrofluid aimed to enrich for endothelial cells ${ }^{16-18}$. In order to evaluate whereas there is a leukocyte carry-over relative to the total leukocytes of the patients as counted by a complete blood count ( $\mathrm{CBC}$ ), the Spearman's Rho correlation coefficients $\rho$ among all different $\mathrm{CBC}$-based parameters and CTC and CEC isolated events were estimated. The results are summarized in Supplemental Table S8.2. Even if many correlations were significant, most of them were very weak. Only CTCs were strongly associated with tdEVs.

\subsubsection{Association of CTCs, tdEVs, CECs and edEVs with progression-free and overall survival of colorectal cancer patients before the initiation of a new therapy}

To determine whether CECs and edEVs associate with PFS and OS, the HRs (95\% CI) were calculated over a wide range of cut-off values for CECs and edEVs of 395 patients as illustrated in Supplemental Figure S8.2. Only 1.8\% of the possible CEC cut-off values can significantly dichotomize patients based on the HR for PFS (Figure S8.2A), and none of these values can result in a patient dichotomization with a significant $\mathrm{HR}$ for OS. On the contrary (Figure S8.2B), significant HRs are obtained over a wide range of edEV values $(76.9 \%$ and $69.8 \%$ of all possible cutoff points based on their PFS and OS, respectively). The optimal cut-off value of edEVs was defined as the one leading to the dichotomization of the patients with the most significant split (min p-value, logrank) based on their HR for OS, which was found to be 287 .

Association of CTCs with PFS and OS and of tdEVs with OS has been reported earlier ${ }^{1,2,5}$. Here, we demonstrate the association of PFS (Figure 8.3) and OS (Figure 8.4) of 395 mCRC patients with CTCs (Panels A), tdEVs (Panels B), CECs (Panels C) and edEVs (Panel D) generating Kaplan Meier survival plots. 

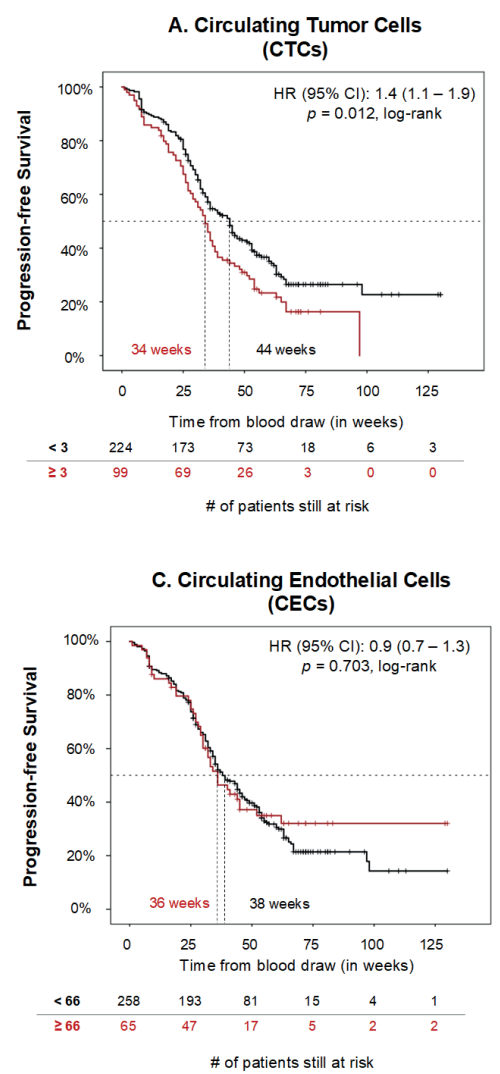

\section{E. Combined CTCs and edEVs}

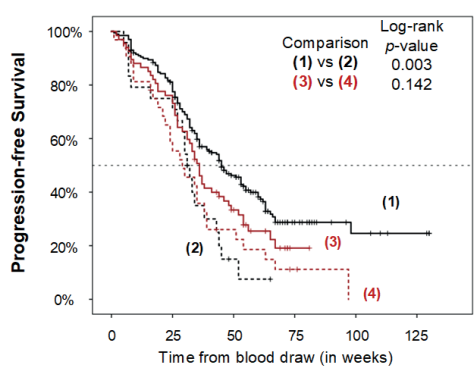

\begin{tabular}{|c|c|c|c|c|c|c|}
\hline $\begin{array}{l}<3 \text { CTCs } \\
<287 \\
\text { edEVs (1) }\end{array}$ & 200 & 154 & 71 & 17 & 6 & 3 \\
\hline $\begin{array}{c}<3 \text { CTCs } \\
\geq 287 \\
\text { edEVs (2) }\end{array}$ & 24 & 17 & 2 & 0 & 0 & 0 \\
\hline $\begin{array}{c}\geq 3 \mathrm{CTCs} \\
<287 \\
\operatorname{edEVs}(3)\end{array}$ & 67 & 50 & 18 & 1 & 0 & 0 \\
\hline $\begin{array}{l}\geq 3 \mathrm{CTCs} \\
\geq 287 \\
\text { edEVs (4) }\end{array}$ & 32 & 17 & 7 & 2 & 0 & 0 \\
\hline
\end{tabular}

B. Tumor-derived Extracellular Vesicles (tdEVs)

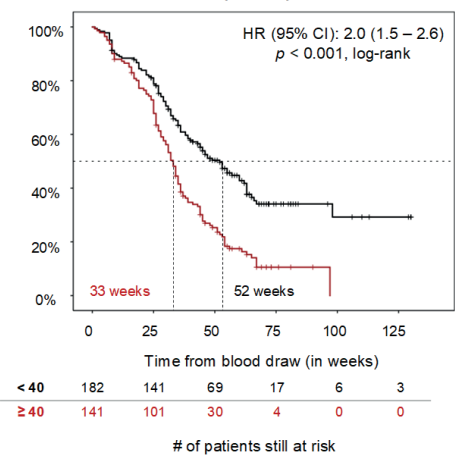

D. Endothelium-derived Extracellular Vesicles (edEVs)

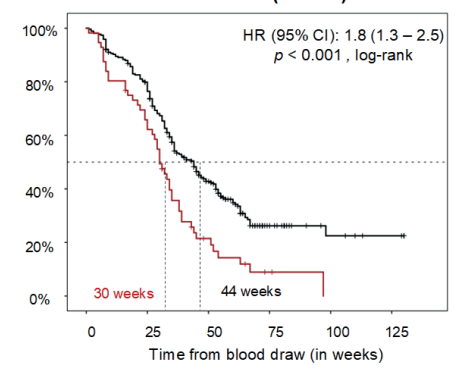

$\begin{array}{lcccccc}<287 & 267 & 205 & 89 & 18 & 6 & 3 \\ \geq 287 & 56 & 35 & 9 & 2 & 0 & 0\end{array}$

\# of patients still at risk

\section{F. Combined tdEVs and edEVs}

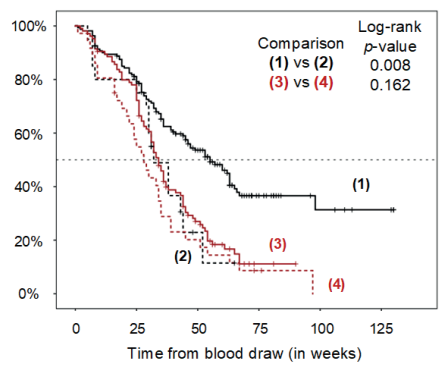

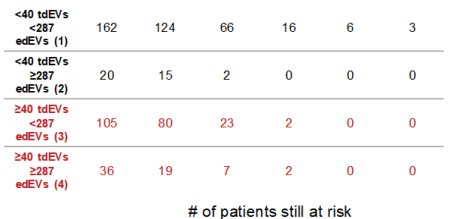

Figure 8.3. Association of CTCs (Panel A), tdEVs (Panel B), CECs (Panel C) and edEVs (Panel D) with progression-free survival of $323 \mathrm{mCRC}$ patients before the initiation of a new therapy. 


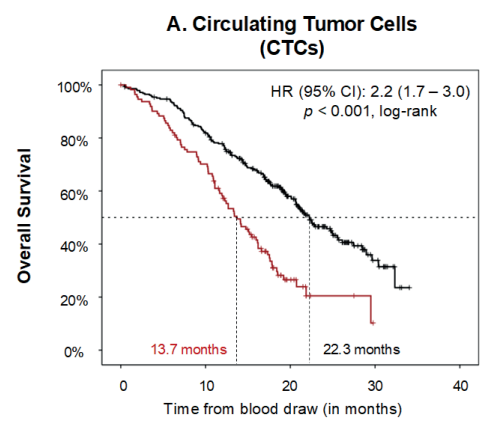

\section{B. Tumor-derived Extracellular Vesicles}

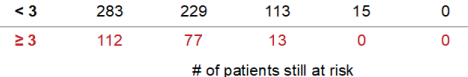

C. Circulating Endothelial Cells (CECs)
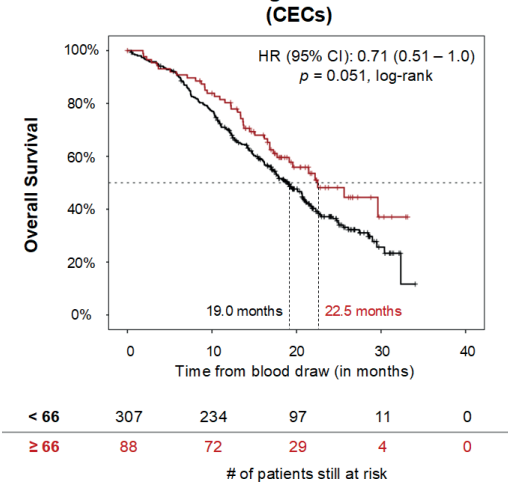

E. Combined CTCs and edEVs

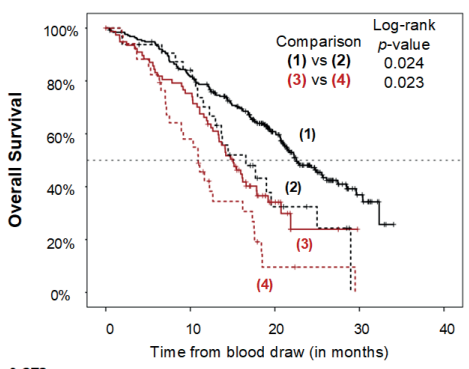

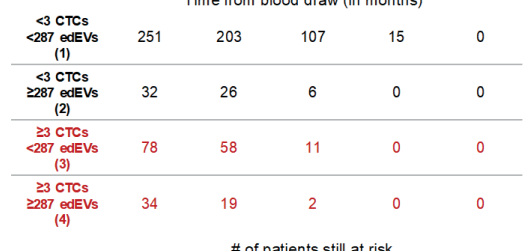
(tdEVs)

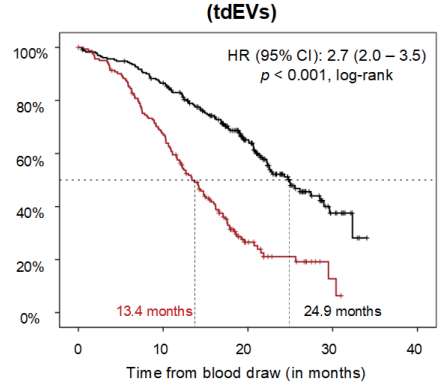

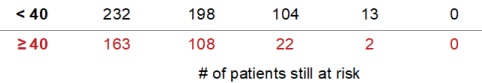

D. Endothelium-derived Extracellular Vesicles (edEVs)

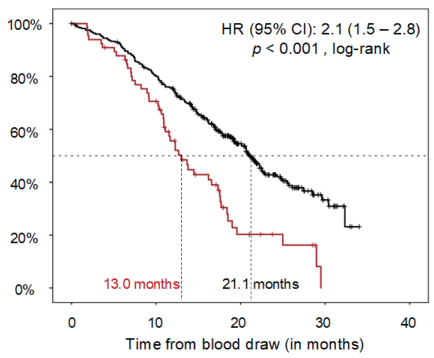

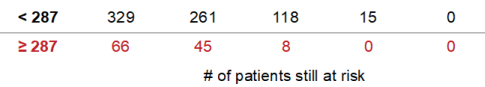

\section{F. Combined tdEVs and edEVs}

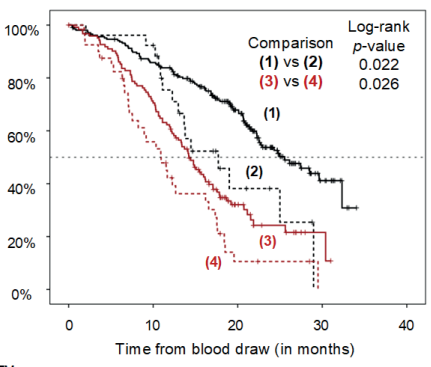

\begin{tabular}{|c|c|c|c|c|c|}
\hline $\begin{array}{l}<40 \text { tdEVs } \\
<287 \text { edEVs } \\
\text { (1) }\end{array}$ & 206 & 174 & 99 & 13 & 0 \\
\hline $\begin{array}{l}<40 \text { tdEVs } \\
\geq 287 \text { edEVs } \\
\text { (2) }\end{array}$ & 26 & 24 & 5 & 0 & 0 \\
\hline $\begin{array}{l}\geq 40 \text { tdEVS } \\
<287 \text { edEVS } \\
\text { (3) }\end{array}$ & 123 & 87 & 19 & 2 & 0 \\
\hline $\begin{array}{c}\geq 40 \text { tdEVs } \\
\geq 287 \text { edEVS } \\
\text { (4) }\end{array}$ & 40 & 21 & 2 & 0 & 0 \\
\hline
\end{tabular}

\# of patients still at risk

Figure 8.4. Association of CTCs (Panel A), tdEVs (Panel B), CECs (Panel C) and edEVs (Panel D) with overall survival of $395 \mathrm{mCRC}$ patients before the initiation of a new therapy. 
Patients were dichotomized using a cut-off of 3 or more CTCs, 40 or more tdEVs, 66 or more CECs (vertical line Supplemental Figure S8.2A) and 287 or more edEVs (vertical line Supplemental Figure S8.2B). It is clear that next to CTCs and tdEVs, also edEVs but not CECs are associated with relatively worse PFS and OS. To evaluate whether edEVs could aid in the prognostication of $\mathrm{mCRC}$ patients with favorable and unfavorable CTC and tdEV counts, we generated ROC curves for CTCs, tdEVs, edEVs and the combined use of CTCs or tdEVs with edEVs (Supplemental Figure S8.3) using as a claffication variable the survival of patients dichotomized by the median OS. The AUC of the combined use of CTCs or tdEVs and edEVs was significantly higher $(p<0.05$, DeLong) as compared to the AUC of solely one biomarker. Kaplan Meier survival curves of PFS and OS were generated stratifying patients in 4 different risk groups after combining their edEV and CTC (Figures 8.3E and 8.4E) or tdEV (Figures 8.3F and $8.4 \mathrm{~F}$ ) counts. Importantly, patients with unfavorable edEV counts and favorable CTC or tdEV counts have significantly shorter PFS and OS compared to patients with both favorable their edEV and CTC or tdEV counts. In case of patients with unfavorable CTC or tdEV counts, edEV counts can further stratify patients only with respect to OS.

\subsubsection{Univariable and multivariable associations between potential risk factors and clinical outcome (PFS and OS) of MCRC patients}

All parameters, including patient characteristics (age, gender, ECOG status, prior adjuvant therapy, more than 1 affected organs, treatment arm), baseline serum $\mathrm{LDH}, \mathrm{CBC}$-based parameters (absolute leukocyte counts, absolute platelet counts, haemoglobin) and all different classes isolated with the CTC and CEC kits (CTCs, tdEVs, CECs, edEVs, leukocytes, nucleated events, ldEVs, CD105+ leukocytes and CD105+ ldEVs) were evaluated as potential risk factors of PFS and OS using univariable cox proportional hazards regression analysis. Next to the continuous log transformed CTCs, tdEVs, CECs and edEVs, the dichotomized ones were also included in the analysis and shown in Table 8.1. 
Table 8.1. Univariable cox proportional hazards regression analysis of $395 \mathrm{mCRC}$ patients

\begin{tabular}{|c|c|c|c|c|c|c|c|c|c|}
\hline \multirow{3}{*}{$\begin{array}{l}\text { Variable } \\
\text { Prior adjuvant therapy }\end{array}$} & \multirow[t]{3}{*}{ Cut-off } & \multicolumn{4}{|c|}{ PFS risk } & \multicolumn{4}{|c|}{ OS risk } \\
\hline & & \multirow{2}{*}{$\begin{array}{c}\text { HR } \\
0.7\end{array}$} & \multicolumn{2}{|c|}{$95 \% \mathrm{CI}$} & \multirow{2}{*}{$\frac{\text { p-value }}{0.043}$} & \multirow{2}{*}{$\begin{array}{c}\text { HR } \\
0.7\end{array}$} & \multicolumn{2}{|c|}{$95 \% \mathrm{CI}$} & \multirow{2}{*}{$\frac{\text { p-value }}{0.046}$} \\
\hline & & & 0.4 & 1.0 & & & 0.4 & 1.0 & \\
\hline $\begin{array}{l}\text { More than } 1 \text { affected } \\
\text { organs }\end{array}$ & & 1.3 & 1.0 & 1.8 & 0.039 & 1.3 & 1.0 & 1.7 & 0.065 \\
\hline BL serum LDH & $\begin{array}{l}\text { above } \\
\text { normal }\end{array}$ & 1.2 & 0.9 & 1.5 & 0.242 & 1.6 & 1.2 & 2.1 & 0.001 \\
\hline Treatment arm & & 1.1 & 0.9 & 1.5 & 0.397 & 1.2 & 0.9 & 1.5 & 0.313 \\
\hline ECOG status & & 1.4 & 1.1 & 1.8 & 0.012 & 1.4 & 1.1 & 1.9 & 0.006 \\
\hline age & & 1.013 & 0.999 & 1.028 & 0.078 & 1.008 & 0.993 & 1.022 & 0.290 \\
\hline gender & & 1.0 & 0.7 & 1.3 & 0.958 & 1.0 & 0.7 & 1.3 & 0.773 \\
\hline \multicolumn{10}{|l|}{ Cell Search } \\
\hline $\begin{array}{l}\text { CTCs } \\
\text { (log transformed) }\end{array}$ & - & 1.2 & 1.1 & 1.4 & 0.004 & 1.6 & 1.4 & 1.9 & $<0.001$ \\
\hline$\geq 3 \mathrm{CTCs}$ & 3 & 1.4 & 1.1 & 1.9 & 0.012 & 2.2 & 1.7 & 3.0 & $<0.001$ \\
\hline $\begin{array}{l}\text { tdEVs } \\
\text { (log transformed) }\end{array}$ & - & 1.8 & 1.4 & 2.3 & $<0.001$ & 2.5 & 2.0 & 3.2 & $<0.001$ \\
\hline$\geq 40 \mathrm{tdEVs}$ & 40 & 2.0 & 1.5 & 2.6 & $<0.001$ & 2.7 & 2.0 & 3.5 & $<0.001$ \\
\hline $\begin{array}{l}\text { CECs } \\
\text { (log transformed) }\end{array}$ & - & 1.1 & 0.9 & 1.5 & 0.338 & 1.0 & 0.8 & 1.2 & 0.767 \\
\hline$\geq 66$ CECs & 66 & 0.9 & 0.7 & 1.3 & 0.703 & 0.71 & 0.51 & 1.0 & 0.051 \\
\hline $\begin{array}{l}\text { edEVs } \\
\text { (log transformed) }\end{array}$ & - & 1.7 & 1.2 & 2.5 & 0.004 & 1.9 & 1.3 & 2.8 & 0.002 \\
\hline$\geq 287 \mathrm{edEVs}$ & 287 & 1.8 & 1.3 & 2.5 & $<0.001$ & 2.1 & 1.5 & 2.8 & $<0.001$ \\
\hline $\begin{array}{l}\text { Other CellSearch } \\
\text { populations }\end{array}$ & & & & & & & & & \\
\hline $\begin{array}{l}\text { Leukocytes }^{\mathrm{a}} \\
\text { (log transformed) }\end{array}$ & - & 1.3 & 1.1 & 1.5 & 0.009 & 1.3 & 1.1 & 1.6 & 0.001 \\
\hline $\begin{array}{l}\operatorname{ldEVs}^{\mathrm{a}} \\
\text { (log transformed) }\end{array}$ & - & 1.1 & 0.9 & 1.4 & 0.437 & 1.2 & 0.9 & 1.5 & 0.219 \\
\hline $\begin{array}{l}\text { Nucleated }^{a} \\
\text { (log transformed) }\end{array}$ & - & 1.1 & 0.9 & 1.4 & 0.239 & 1.3 & 1.0 & 1.6 & 0.048 \\
\hline $\begin{array}{l}\text { Leukocytes }^{\mathrm{b}} \\
\text { (log transformed) }\end{array}$ & - & 1.1 & 0.8 & 1.5 & 0.737 & 1.1 & 0.8 & 1.5 & 0.751 \\
\hline $\begin{array}{l}\text { ldEVs }{ }^{\mathrm{b}} \\
\text { (log transformed) }\end{array}$ & - & 1.0 & 0.7 & 1.4 & 0.976 & 1.1 & 0.8 & 1.6 & 0.608 \\
\hline $\begin{array}{l}\text { Nucleated }^{b} \\
\text { (log transformed) }\end{array}$ & - & 1.1 & 0.9 & 1.5 & 0.436 & 1.3 & 1.0 & 1.7 & 0.091 \\
\hline
\end{tabular}




\begin{tabular}{|c|c|c|c|c|c|c|c|c|c|}
\hline \multirow{3}{*}{$\begin{array}{l}\text { Variable } \\
\text { CD105+ leukocytes } \\
\text { (log transformed) }\end{array}$} & \multirow{3}{*}{$\begin{array}{l}\text { Cut-off } \\
-\end{array}$} & \multicolumn{4}{|c|}{ PFS risk } & \multicolumn{4}{|c|}{ OS risk } \\
\hline & & \multirow{2}{*}{$\frac{\text { HR }}{1.0}$} & \multicolumn{2}{|c|}{$95 \% \mathrm{CI}$} & \multirow{2}{*}{$\frac{\text { p-value }}{0.789}$} & \multirow{2}{*}{$\frac{\text { HR }}{1.0}$} & \multicolumn{2}{|c|}{$95 \%$ CI } & \multirow{2}{*}{$\frac{\text { p-value }}{0.927}$} \\
\hline & & & 0.9 & 1.2 & & & 0.8 & 1.2 & \\
\hline $\begin{array}{l}\text { CD105+ ldEVs }{ }^{b} \\
\text { (log transformed) }\end{array}$ & - & 1.0 & 0.8 & 1.2 & 0.842 & 1.1 & 0.9 & 1.4 & 0.203 \\
\hline $\begin{array}{l}\text { CBC-based } \\
\text { parameters }\end{array}$ & & & & & & & & & \\
\hline $\begin{array}{l}\text { Leukocytes } \\
\text { (log transformed) }\end{array}$ & - & 6.0 & 2.1 & 16.9 & 0.001 & 9.5 & 3.5 & 25.9 & $<0.001$ \\
\hline $\begin{array}{l}\text { Platelets } \\
\text { (log transformed) }\end{array}$ & - & 4.0 & 1.6 & 10.3 & 0.004 & 5.3 & 2.1 & 13.4 & $<0.001$ \\
\hline Hemoglobin, per 1g/dL & - & 0.8 & 0.7 & 0.9 & $<0.001$ & 0.7 & 0.6 & 0.8 & $<0.001$ \\
\hline
\end{tabular}

${ }^{a}$ refers to events co-isolated with the CTC kit, ${ }^{b}$ refers to events co-isolated with the CEC kit

Surprisingly, leukocytes present in the cartridges after EpCAM enrichment showed a significant association with PFS and OS; the nucleated events after EPCAM enrichment were also significantly associated with OS (not PFS). In contrast to EpCAM enriched leukocytes and nucleated events, the respective populations after CD146 enrichment were not significantly associated with worse PFS or OS. For the multivariable analysis, the significant predictors of the univariable analysis were included. tdEVs, CBC-based leukocytes, haemoglobin and surprisingly, leukocytes of the EpCAM enriched samples remained significant predictors of both PFS and OS (Table 8.2). edEVs remained a significant independent predictor of OS, but not of PFS, in the final multivariable model.

Table 8.2. Multivariable cox proportional hazards regression analysis of $\mathrm{mCRC}$ patients using backward stepwise elimination

\begin{tabular}{|c|c|c|c|c|c|c|c|c|}
\hline \multirow{3}{*}{$\begin{array}{l}\text { Variables in equation } \\
\geq 40 \mathrm{tdEVs}\end{array}$} & \multicolumn{4}{|c|}{ Progression-free Survival } & \multicolumn{4}{|c|}{ Overall Survival } \\
\hline & \multirow{2}{*}{$\frac{\text { HR }}{1.6}$} & \multicolumn{2}{|c|}{$95 \% \mathrm{CI}$} & \multirow{2}{*}{$\frac{\text { p-value }}{0.001}$} & \multirow{2}{*}{$\frac{\mathrm{HR}}{2.2}$} & \multicolumn{2}{|c|}{$95 \% \mathrm{CI}$} & \multirow{2}{*}{$\frac{\text { p-value }}{<0.001}$} \\
\hline & & 1.2 & 2.2 & & & 1.6 & 2.9 & \\
\hline$\geq 287$ edEVs & & & & & 1.4 & 1.0 & 2.0 & 0.059 \\
\hline Haemoglobin, per $1 \mathrm{~g} / \mathrm{dL}$ & 0.9 & 0.8 & 1.0 & 0.022 & 0.9 & 0.7 & 1.0 & 0.024 \\
\hline Leukocytes $^{\text {a }}$ (log transformed) & 1.2 & 1.0 & 1.5 & 0.058 & 1.2 & 1.0 & 1.5 & 0.044 \\
\hline $\begin{array}{l}\text { CBC Leukocytes } \\
\text { (log transformed) }\end{array}$ & 3.3 & 1.1 & 9.9 & 0.033 & 3.6 & 1.3 & 10.3 & 0.015 \\
\hline More than 1 organ affected & 1.3 & 1.0 & 1.7 & 0.056 & & & & \\
\hline
\end{tabular}




\subsection{Discussion}

Liquid biopsies have attracted the interest of many research groups thanks to their potential to improve disease management of cancer patients in a non-invasive and timely fashion. Circulating tumor cells and recently, tumor-derived extracellular vesicles have emerged as significant predictors of overall survival of castrationresistant prostate, metastatic breast, metastatic colorectal and non-small cell lung cancer patients ${ }^{1,3-5,19,20}$. Importantly, their phenotypic and genotypic characterization can shed light into new therapeutic targets and predict the treatments that will lead to the recession of specific patient groups ${ }^{21,22}$. However, the scarceness of CTCs in blood, their frequently apoptotic status and the leukocyte carry-over after the chosen enrichment technique impedes their downstream genetic analysis ${ }^{23,24}$. Therefore, complementary research is being conducted towards the discovery of additional biomarkers predictive of treatment benefit.

Towards that direction, circulating endothelial cells hold great promise since their elevated counts could be an indicator of tumor angiogenesis and/or vascular damage due to the tumor growth; thus, it could predict patient response to anti-angiogenic treatments. A variety of assays for CEC enumeration have been reported; however, the lack of consensus on CEC phenotype and standardized techniques for their isolation and detection hinders the comparison of results among different studies and the drawing of incontrovertible conclusions ${ }^{25}$. The CEC assay that we used in the present study, immunomagnetically enriched CD146+ CECs from $4 \mathrm{~mL}$ of whole blood and identified them based on DAPI, expression of CD105 and lack of CD45. CEC frequencies in blood of cancer patients are elevated compared to CEC frequencies in healthy donors ${ }^{6}$. However, no association with clinical outcome could be observed in metastatic prostate cancer ${ }^{26}$ and $\mathrm{mCRC}^{7}$. One explanation is that CEC counts are biased due to the endothelial cells detached during the blood draw ${ }^{6}$. A solution to eliminate that bias could be the use of antibodies targeting exclusively cancer-associated CECs ${ }^{27}$. However, in a study in which CECs were defined as DAPI+, CD34+, CD146+, CD45- and also expressed the immune check molecule CD276, which is frequently associated with cancer, no association could be found with poor outcome or treatment responses ${ }^{28}$.

As tdEVs strongly correlate with CTCs and are similarly associated with clinical outcome, we asked ourselves whether edEVs could also be detected in blood samples enriched for CECs and whether their presence is associated with clinical outcome. In contrast to CEC counts, which are influenced by a portion of endothelial cells originating from the vascular wall due to the blood draw, edEV count is expected to be less biased. We investigated this by analysis of the fluorescence images of samples from 395 mCRC patients processed with both CEC and CTC Cellsearch kits in a standardized manner. The recently introduced open-source ACCEPT software allowed 
for the automated identification and enumeration of different classes of objects that were co-isolated with the two kits ${ }^{29}$. This included the EVs derived from cancer cells, endothelial cells and leukocytes ${ }^{3,30}$.

Previous studies have reported the presence of edEVs in different pathophysiological conditions as analyzed mainly with flow cytometry ${ }^{31-33}$. However, the need of a standardized isolation technique and a consensus on their definition has been stressed in several studies ${ }^{34,35}$. Duval et al. compared different antibody combinations using flow cytometry and concluded that $\mathrm{CD} 146+, \mathrm{CD} 105+, \mathrm{CD} 45$ - definition results in the most efficient detection of edEV population ${ }^{31}$. In consistency to their probing, in our study, CD146 was used to immunomagnetically enrich for CECs and edEVs, CD105 was used as an inclusion marker and $\mathrm{CD} 45$ as an exclusion marker to discriminate from the leukocyte carry-over. However, only the large edEVs with a diameter larger than $1 \mu \mathrm{m}$ are reported here, since the plasma fraction is by default discarded by the Cellsearch system.

The frequencies of all different classes isolated with both kits as well as the originally manual counts of CTCs and CECs, are summarized in Figures 8.2 and Supplemental Figure S8.1. It is noteworthy that EVs of tumor cell and endothelial cell origin were found at approximately an order of magnitude higher frequencies in the blood of mCRC patients compared to the respective CTC and CEC frequencies, whereas the frequencies of ldEVs were in both cases an order of magnitude lower frequencies compared to the respective leukocyte counts. In case of the EPCAM (CTC) enriched samples, a rational explanation could be that the leukocyte carry over is non-specific and possibly associated with increased Fc receptors on their surfaces that lead to their binding to the antibody against EpCAM conjugated to the ferrofluid. These receptors will be reduced since they should be proportional to the available surface of the EVs resulting to lower co-isolated ldEV counts. However, the leukocyte carryover in case of the CD146 (CEC) enriched samples is also specific, as CD146 is not only expressed on CECs but also on a subset of activated T lymphocytes ${ }^{16-18}$. Notably, the ldEVs isolated with the CEC kit are also fewer compared to the co-isolated leukocytes suggesting a different underlying biology depending on the cell type. Either leukocytes secrete much fewer EVs compared to tumor and endothelial cells or EVs secreted by leukocytes have such a size and density that leaves them in the plasma fraction after centrifugation at $800 \mathrm{~g}$ for $10 \mathrm{~min}$ which is not processed with the CellSearch system. Further research is needed to elucidate the frequencies, density and size range of EVs of different origins found in the circulation of healthy donors and cancer patients. The relative frequencies of CTCs to the other blood cells in different cancer types is already known; however that is not the case for tdEVs ${ }^{36}$. It may be that tdEVs outnumber EVs of different origins or at least their relative frequencies to the different $\mathrm{EV}$ origins are higher than CTCs because of their active role in metastasis ${ }^{37}$. 
We assessed the association of all different classes co-isolated after CTC and CEC enrichment with the clinical outcome of patients. For edEVs, we surprisingly noticed that when plotting all possible cut-off values of edEV counts versus the HR, more than half of the values could significantly dichotomize the patients into a more and less favorable group of PFS ( $76.9 \%$ of cut-off values) and OS ( $69.8 \%$ of cut-off values) (Supplemental Figure S8.2). This was into contrast with CECs, for which only $1.8 \%$ of possible cut-off values were significant in case of only PFS. The cutoff value of $287 \mathrm{edEVs}$ in $4 \mathrm{~mL}$ of peripheral blood was chosen as the value leading to the most significant split of the patients. The group of patients with elevated edEVs experienced significantly shorter median PFS and OS (Figures 8.3 and 8.4) compared to the patients with $<287$ edEVs. Importantly, edEVs and tdEVs remained independent predictors of OS in the final multivariable model. That can be also visualized in the Kaplan Meier survival plots where patients were split by combining their tdEV and edEV counts (Figures 8.3 and 8.4) as well as in the ROC curves (Supplemental Figure S8.3). This observation suggests that the metastatic process is associated with an active release of edEVs, which may be contributed to the penetration of cancer cells through the vasculature and/or the active growth of blood vessels in the primary and metastatic sites. In this study, both treatment arms received the angiogenesis inhibitor bevacizumab, which does not allow us to determine a possible effect of the drug on the edEV release. No difference in the edEV counts could be detected in patients in which the EGFR inhibitor Cetuximab was administered or not.

Previously, we reported the presence of a significantly larger number of leukocytes and nucleated cells of unknown origin after EpCAM enrichment in blood of NSCLC patients as compared to healthy donors ${ }^{38}$. The majority of these nucleated cells are granulocytes which express less CD45 compared to lymphocytes and are not detected by the CellTracks Analyzer ${ }^{38}$. Here, we unexpectedly noticed a significant association between PFS and OS of MCRC patients with increased counts of leukocytes co-isolated after EpCAM enrichment. In contrast, no association with clinical outcome could be found between the carried over leukocytes using the CD146 enrichment (Table 8.1). That observation raised questions about the subtype of leukocytes isolated with the CTC kit. Up to date, leukocytes were considered to constitute a non-specific carry over. When evaluating the relationship between the leukocyte counts in the EPCAMenriched samples and the leukocyte counts from the available complete blood counts $(\mathrm{CBC})$, a weak correlation could be found using the Spearman's Rho test (Supplemental Table S8.1). That finding rejects a reasonable explanation that the leukocyte carry-over reflects the total leukocyte count of the patients, which is already associated with their clinical outcome (Table 8.1); an observation, which is also consistent with findings of other studies in bladder, prostate and non-small cell lung cancer ${ }^{39-42}$. Therefore, these clinically relevant leukocytes either overexpress Fc $\gamma$ receptors that bind to the IgG immunoglobulins of the EPCAM ferrofluid, or they express EPCAM. 
Fc receptors are expressed on all haematopoetic cells playing a key role on the immune modulation having both inhibitory and activating functions ${ }^{43,44}$. The increased expression of $\mathrm{Fc} \gamma$ receptors on the monocytes of gastric cancer patients has been previously reported ${ }^{45}$. However, the phenotypic characterization of not only monocytes but of all different leukocyte populations regarding the expression profile of their different classes of Fc $\gamma$ receptors (FC $\gamma$ RI, FC $\gamma$ RII, FC $\gamma$ RIII and FC $\gamma$ RIV) and their roles on the immune responses of the cancer patients is emerging urgent. Depending on the affinity of immunoglobulins IgG to the different Fc receptors, cells can have differentiated immune functions ${ }^{46}$. With that as a basis, novel antibodies with a selectively high affinity with the activating Fc receptors of the immune cells can be designed and developed towards improved immuno-therapies ${ }^{47}$.

An alternative plausible explanation would be the expression of EpCAM on a subset of leukocytes and their specific isolation using the CTC kit. EPCAM belongs in the CAM families serving a diversity of cell functions ${ }^{48}$. Its expression is known to be restricted only in epithelial cells in healthy humans. De novo expression of EpCAM has been reported in mature human hepatocytes during various inflammatory liver diseases and liver regeneration and repair ${ }^{49,50}$. Furthermore, EPCAM is expressed also by a subset of thymocytes, $\mathrm{B}$ and $\mathrm{T}$ lymphocytes in mice facilitating their infiltration in inflammation sites ${ }^{51,52}$. Similarly, the upregulation of integrins, which comprise a CAM family, by effector $\mathrm{T}$ lymphocytes also facilitate their targeted access to inflammatory sites ${ }^{53}$. However, to our knowledge it has not been investigated whereas EPCAM is expressed in any subsets of leukocytes in cancer patients. The EpCAM expression on the surfaces of the leukocyte could originate/emanate either by the activation of originally silent molecular pathways or by the uptake of tumor-derived extracellular vesicles. Different mechanisms of EV internalization have been described with the cell-EV fusion resulting to the fusion of the two membranes ${ }^{54}$; consequently, the antigens of the merged EV should end up in the cell membrane. Additionaly, the functional exchange of mRNAs between cells via EVs has been demonstrated ${ }^{55}$. Last but not least, it has been demonstrated in murine models that tdEVs accumulate in all leukocyte subpopulations altering the phenotypes of the recipient cells ${ }^{56,57}$.

In conclusion, this study shows that endothelial-derived extracellular vesicles isolated based on their CD146 expression and detected based on their CD105 expression are predictors of progression-free and overall survival in metastatic colorectal cancer and improve the risk stratification of patients based on solely their CTC or tdEV counts. Interestingly, the EpCAM-based co-isolated leukocytes remain an independent predictor in the final multivariable model suggesting further investigation on the phenotype of that population. 


\section{References}

1. Cohen SJ, Punt CJ, Iannotti N, Saidman BH, Sabbath KD, Gabrail NY et al. Relationship of circulating tumor cells to tumor response, progression-free survival, and overall survival in patients with metastatic colorectal cancer. J Clin Oncol 2008; 26(19): 3213-3221; doi 10.1200/ JCO.2007.15.8923.

2. Tol J, Koopman M, Miller MC, Tibbe A, Cats A, Creemers GJM et al. Circulating tumour cells early predict progression-free and overall survival in advanced colorectal cancer patients treated with chemotherapy and targeted agents. Annals of oncology : official journal of the European Society for Medical Oncology 2010; 21(5): 1006-1012.

3. Nanou A, Coumans FAW, van Dalum G, Zeune LL, Dolling D, Onstenk W et al. Circulating tumor cells, tumor-derived extracellular vesicles and plasma cytokeratins in castrationresistant prostate cancer patients. Oncotarget 2018; 9(27): 19283-19293; doi 10.18632/ oncotarget.25019.

4. de Wit S, Rossi E, Weber S, Tamminga M, Manicone M, Swennenhuis JF et al. Single tube liquid biopsy for advanced non-small cell lung cancer. Int J Cancer 2018; doi 10.1002/ijc.32056.

5. Nanou A, Miller MC, Zeune LL, de Wit S, Punt CJA, Groen HJM et al. Tumour-derived extracellular vesicles in blood of metastatic cancer patients associate with overall survival. $\mathrm{Br}$ J Cancer 2020; doi 10.1038/s41416-019-0726-9.

6. Rowand JL, Martin G, Doyle GV, Miller MC, Pierce MS, Connelly MC et al. Endothelial cells in peripheral blood of healthy subjects and patients with metastatic carcinomas. Cytometry $\mathrm{A}$ 2007; 71(2): 105-113; doi 10.1002/cyto.a.20364.

7. Simkens LH, Tol J, Terstappen LW, Teerenstra S, Punt CJ, Nagtegaal ID. The predictive and prognostic value of circulating endothelial cells in advanced colorectal cancer patients receiving first-line chemotherapy and bevacizumab. Ann Oncol 2010; 21(12): 2447-2448; doi 10.1093/annonc/mdq640.

8. Tol J, Koopman M, Cats A, Rodenburg CJ, Creemers GJM, Schrama JG et al. Chemotherapy, bevacizumab, and cetuximab in metastatic colorectal cancer. The New England journal of medicine 2009; 360(6): 563-572.

9. Therasse P, Arbuck SG, Eisenhauer EA, Wanders J, Kaplan RS, Rubinstein L et al. New guidelines to evaluate the response to treatment in solid tumors. European Organization for Research and Treatment of Cancer, National Cancer Institute of the United States, National Cancer Institute of Canada. Journal of the National Cancer Institute 2000; 92(3): 205-216.

10. Tol J, Koopman M, Rodenburg CJ, Cats A, Creemers GJ, Schrama JG et al. A randomised phase III study on capecitabine, oxaliplatin and bevacizumab with or without cetuximab in firstline advanced colorectal cancer, the CAIRO2 study of the Dutch Colorectal Cancer Group (DCCG). An interim analysis of toxicity. Annals of oncology : official journal of the European Society for Medical Oncology 2008; 19(4): 734-738.

11. Coumans F, Terstappen L. Detection and Characterization of Circulating Tumor Cells by the CellSearch Approach. Methods Mol Biol 2015; 1347: 263-278; doi 10.1007/978-1-4939-2990-0_18. 
12. Budczies J, Klauschen F, Sinn BV, Gyorffy B, Schmitt WD, Darb-Esfahani S et al. Cutoff Finder: a comprehensive and straightforward Web application enabling rapid biomarker cutoff optimization. Plos One 2012; 7(12): e51862; doi 10.1371/journal.pone.0051862.

13. DeLong ER, DeLong DM, Clarke-Pearson DL. Comparing the areas under two or more correlated receiver operating characteristic curves: a nonparametric approach. Biometrics 1988; 44(3): 837-845.

14. O'Connell PJ, McKenzie A, Fisicaro N, Rockman SP, Pearse MJ, d'Apice AJ. Endoglin: a 180-kD endothelial cell and macrophage restricted differentiation molecule. Clin Exp Immunol 1992; 90(1): 154-159.

15. Lastres P, Bellon T, Cabanas C, Sanchez-Madrid F, Acevedo A, Gougos A et al. Regulated expression on human macrophages of endoglin, an Arg-Gly-Asp-containing surface antigen. Eur J Immunol 1992; 22(2): 393-397; doi 10.1002/eji.1830220216.

16. Dignat-George F, Sabatier F, Blann A, Woywodt A. Detection of circulating endothelial cells: CD146-based magnetic separation enrichment or flow cytometric assay? J Clin Oncol 2007; 25(5): e1-2; author reply e3-5; doi 10.1200/JCO.2006.07.7677.

17. Elshal MF, Khan SS, Takahashi Y, Solomon MA, McCoy JP. CD146 (Mel-CAM), an adhesion marker of endothelial cells, is a novel marker of lymphocyte subset activation in normal peripheral blood. Blood 2005; 106(8): 2923-2924.

18. Pickl WF, Majdic O, Fischer GF, Petzelbauer P, Fae I, Waclavicek M et al. MUC18/MCAM (CD146), an activation antigen of human T lymphocytes. J Immunol 1997; 158(5): 2107-2115.

19. Cristofanilli M, Budd GT, Ellis MJ, Stopeck A, Matera J, Miller MC et al. Circulating tumor cells, disease progression, and survival in metastatic breast cancer. N Engl J Med 2004; 351(8): 781-791; doi 10.1056/NEJMoa040766.

20. Scher HI, Heller G, Molina A, Attard G, Danila DC, Jia X et al. Circulating tumor cell biomarker panel as an individual-level surrogate for survival in metastatic castration-resistant prostate cancer. J Clin Oncol 2015; 33(12): 1348-1355; doi 10.1200/JCO.2014.55.3487.

21. Bidard FC, Fehm T, Ignatiadis M, Smerage JB, Alix-Panabieres C, Janni W et al. Clinical application of circulating tumor cells in breast cancer: overview of the current interventional trials. Cancer Metastasis Rev 2013; 32(1-2): 179-188; doi 10.1007/s10555-012-9398-0.

22. El-Heliebi A, Hille C, Laxman N, Svedlund J, Haudum C, Ercan E et al. In situ detection and quantification of AR-V7, AR-FL, PSA, and KRAS point mutations in circulating tumor cells. Clinical chemistry 2018; 64(3): 536-546.

23. Swennenhuis JF, Reumers J, Thys K, Aerssens J, Terstappen LW. Efficiency of whole genome amplification of single circulating tumor cells enriched by CellSearch and sorted by FACS. Genome Med 2013; 5(11): 106; doi 10.1186/gm510.

24. Coumans FA, Ligthart ST, Uhr JW, Terstappen LW. Challenges in the enumeration and phenotyping of CTC. Clin Cancer Res 2012; 18(20): 5711-5718; doi 10.1158/1078-0432.CCR-121585 .

25. Strijbos MH, Gratama JW, Kraan J, Lamers CH, den Bakker MA, Sleijfer S. Circulating endothelial cells in oncology: pitfalls and promises. Br J Cancer 2008; 98(11): 1731-1735; doi 10.1038/sj.bjc. 6604383 . 
26. Strijbos MH, Gratama JW, Schmitz PI, Rao C, Onstenk W, Doyle GV et al. Circulating endothelial cells, circulating tumour cells, tissue factor, endothelin-1 and overall survival in prostate cancer patients treated with docetaxel. Eur J Cancer 2010; 46(11): 2027-2035; doi 10.1016/j.ejca.2010.03.030.

27. Smirnov DA, Foulk BW, Doyle GV, Connelly MC, Terstappen LWMM, O'Hara SM. Global gene expression profiling of circulating endothelial cells in patients with metastatic carcinomas. Cancer research 2006; 66(6): 2918-2922.

28. Gootjes EC, Kraan J, Buffart T, Verhoef C, Verheul HM, Sleijfer S et al. CD276-positive circulating endothelial cells in advanced colorectal cancer. In: American Society of Clinical Oncology; 2019.

29. Zeune L. Automated CTC Classification, Enumeration and Pheno Typing: Where Math meets Biology [PhD dissertation]. Enschede: Medical Cell BioPhysics, University of Twente; 2019.

30. Nanou A, Zeune LL, Terstappen L. Leukocyte-Derived Extracellular Vesicles in Blood with and without EpCAM Enrichment. Cells 2019; 8(8); doi 10.3390/cells8080937.

31. Duval A, Helley D, Capron L, Youinou P, Renaudineau Y, Dubucquoi S et al. Endothelial dysfunction in systemic lupus patients with low disease activity: evaluation by quantification and characterization of circulating endothelial microparticles, role of anti-endothelial cell antibodies. Rheumatology (Oxford) 2010; 49(6): 1049-1055; doi 10.1093/rheumatology/keq041.

32. Dignat-George F, Boulanger CM. The many faces of endothelial microparticles. Arterioscler Thromb Vasc Biol 2011; 31(1): 27-33; doi 10.1161/ATVBAHA.110.218123.

33. Hromada C, Muhleder S, Grillari J, Redl H, Holnthoner W. Endothelial Extracellular VesiclesPromises and Challenges. Front Physiol 2017; 8: 275; doi 10.3389/fphys.2017.00275.

34. Jy W, Horstman LL, Jimenez JJ, Ahn YS, Biro E, Nieuwland R et al. Measuring circulating cell-derived microparticles. J Thromb Haemost 2004; 2(10): 1842-1851; doi 10.1111/j.15387836.2004.00936.x.

35. Lacroix R, Robert S, Poncelet P, Dignat-George F. Overcoming limitations of microparticle measurement by flow cytometry. Semin Thromb Hemost 2010; 36(8): 807-818; doi 10.1055/s0030-1267034.

36. Coumans F, van Dalum G, Terstappen L. CTC Technologies and Tools. Cytometry A 2018; 93(12): 1197-1201; doi 10.1002/cyto.a.23684.

37. Ciardiello C, Cavallini L, Spinelli C, Yang J, Reis-Sobreiro M, de Candia P et al. Focus on Extracellular Vesicles: New Frontiers of Cell-to-Cell Communication in Cancer. International journal of molecular sciences 2016; 17(2): 175.

38. de Wit S, Zeune LL, Hiltermann TJN, Groen HJM, Dalum GV, Terstappen L. Classification of Cells in CTC-Enriched Samples by Advanced Image Analysis. Cancers (Basel) 2018; 10(10); doi 10.3390/cancers10100377.

39. Bhindi B, Hermanns T, Wei Y, Yu J, Richard PO, Wettstein MS et al. Identification of the best complete blood count-based predictors for bladder cancer outcomes in patients undergoing radical cystectomy. Br J Cancer 2016; 114(2): 207-212; doi 10.1038/bjc.2015.432.

40. Taussky D, Soulieres D, Azoulay L, Yin H, Bahig H, Bahary JP et al. A Combination of Testosterone and White Blood Cell Count as a Predictive Factor of Overall Survival in Localized Prostate Cancer. Target Oncol 2017; 12(5): 695-701; doi 10.1007/s11523-017-0516-3. 
41. Tibaldi C, Vasile E, Bernardini I, Orlandini C, Andreuccetti M, Falcone A. Baseline elevated leukocyte count in peripheral blood is associated with poor survival in patients with advanced non-small cell lung cancer: a prognostic model. J Cancer Res Clin Oncol 2008; 134(10): 1143-1149; doi 10.1007/s00432-008-0378-2.

42. Mandrekar SJ, Schild SE, Hillman SL, Allen KL, Marks RS, Mailliard JA et al. A prognostic model for advanced stage nonsmall cell lung cancer. Pooled analysis of North Central Cancer Treatment Group trials. Cancer-Am Cancer Soc 2006; 107(4): 781-792; doi 10.1002/cncr.22049.

43. Bournazos S, Wang TT, Ravetch JV. The Role and Function of Fcgamma Receptors on Myeloid Cells. Microbiol Spectr 2016; 4(6); doi 10.1128/microbiolspec.MCHD-0045-2016.

44. Pincetic A, Bournazos S, DiLillo DJ, Maamary J, Wang TT, Dahan R et al. Type I and type II Fc receptors regulate innate and adaptive immunity. Nat Immunol 2014; 15(8): 707-716; doi 10.1038/ni.2939.

45. Uracz W, Pituch-Noworolska A, Popiela T, Zembala M. The Fc receptors of normal and cancer patients monocytes. Immunobiology 1982; 163(5): 450-459; doi 10.1016/So171-2985(82)80059-4.

46. Nimmerjahn F, Ravetch JV. Divergent immunoglobulin g subclass activity through selective Fc receptor binding. Science 2005; 310(5753): 1510-1512; doi 10.1126/science.1118948.

47. Stewart R, Hammond SA, Oberst M, Wilkinson RW. The role of Fc gamma receptors in the activity of immunomodulatory antibodies for cancer. Journal for ImmunoTherapy of Cancer 2014 2(1): 29.

48. Trzpis M, McLaughlin PM, de Leij LM, Harmsen MC. Epithelial cell adhesion molecule: more than a carcinoma marker and adhesion molecule. Am J Pathol 2007; 171(2): 386-395; doi 10.2353/ajpath.2007.070152.

49. Breuhahn K, Baeuerle PA, Peters M, Prang N, Tox U, Kohne-Volland R et al. Expression of epithelial cellular adhesion molecule (Ep-CAM) in chronic (necro-)inflammatory liver diseases and hepatocellular carcinoma. Hepatol Res 2006; 34(1): 50-56; doi 10.1016/j. hepres.2005.10.006.

50. de Boer CJ, van Krieken JH, Janssen-van Rhijn CM, Litvinov SV. Expression of Ep-CAM in normal, regenerating, metaplastic, and neoplastic liver. J Pathol 1999; 188(2): 201-206; doi 10.1002/(SICI)1096-9896(199906)188:2<201::AID-PATH339>3.0.CO;2-8.

51. Amann M, Friedrich M, Lutterbuese P, Vieser E, Lorenczewski G, Petersen L et al. Therapeutic window of an EPCAM/CD3-specific BiTE antibody in mice is determined by a subpopulation of EpCAM-expressing lymphocytes that is absent in humans. Cancer Immunol Immunother 2009; 58(1): 95-109; doi 10.1007/s00262-008-0529-y.

52. Nelson AJ, Dunn RJ, Peach R, Aruffo A, Farr AG. The murine homolog of human Ep-CAM, a homotypic adhesion molecule, is expressed by thymocytes and thymic epithelial cells. Eur $\mathrm{J}$ Immunol 1996; 26(2): 401-408; doi 10.1002/eji.1830260220.

53. Dailey MO. Expression of $\mathrm{T}$ lymphocyte adhesion molecules: regulation during antigeninduced T cell activation and differentiation. Crit Rev Immunol 1998; 18(3): 153-184.

54. McKelvey KJ, Powell KL, Ashton AW, Morris JM, McCracken SA. Exosomes: Mechanisms of Uptake. J Circ Biomark 2015; 4: 7; doi 10.5772/61186. 
55. Valadi H, Ekström K, Bossios A, Sjöstrand M, Lee JJ, Lötvall JO. Exosome-mediated transfer of mRNAs and microRNAs is a novel mechanism of genetic exchange between cells. Nature cell biology 2007; 9(6): 654 .

56. Chennakrishnaiah S, Meehan B, D’Asti E, Montermini L, Lee TH, Karatzas N et al. Leukocytes as a reservoir of circulating oncogenic DNA and regulatory targets of tumor-derived extracellular vesicles. Journal of Thrombosis and Haemostasis 2018; 16(9): 1800-1813.

57. Zech D, Rana S, Buchler MW, Zoller M. Tumor-exosomes and leukocyte activation: an ambivalent crosstalk. Cell Commun Signal 2012; 10(1): 37; doi 10.1186/1478-811X-10-37. 


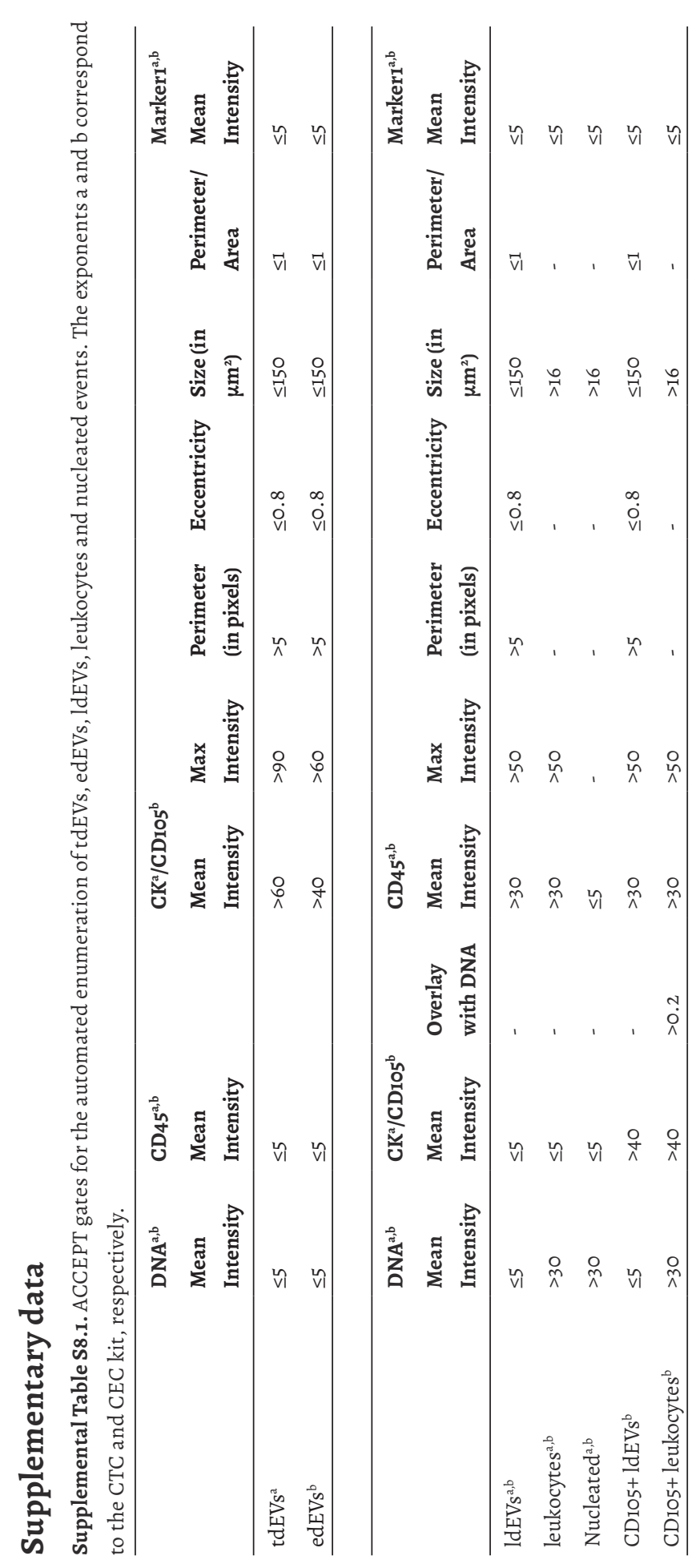




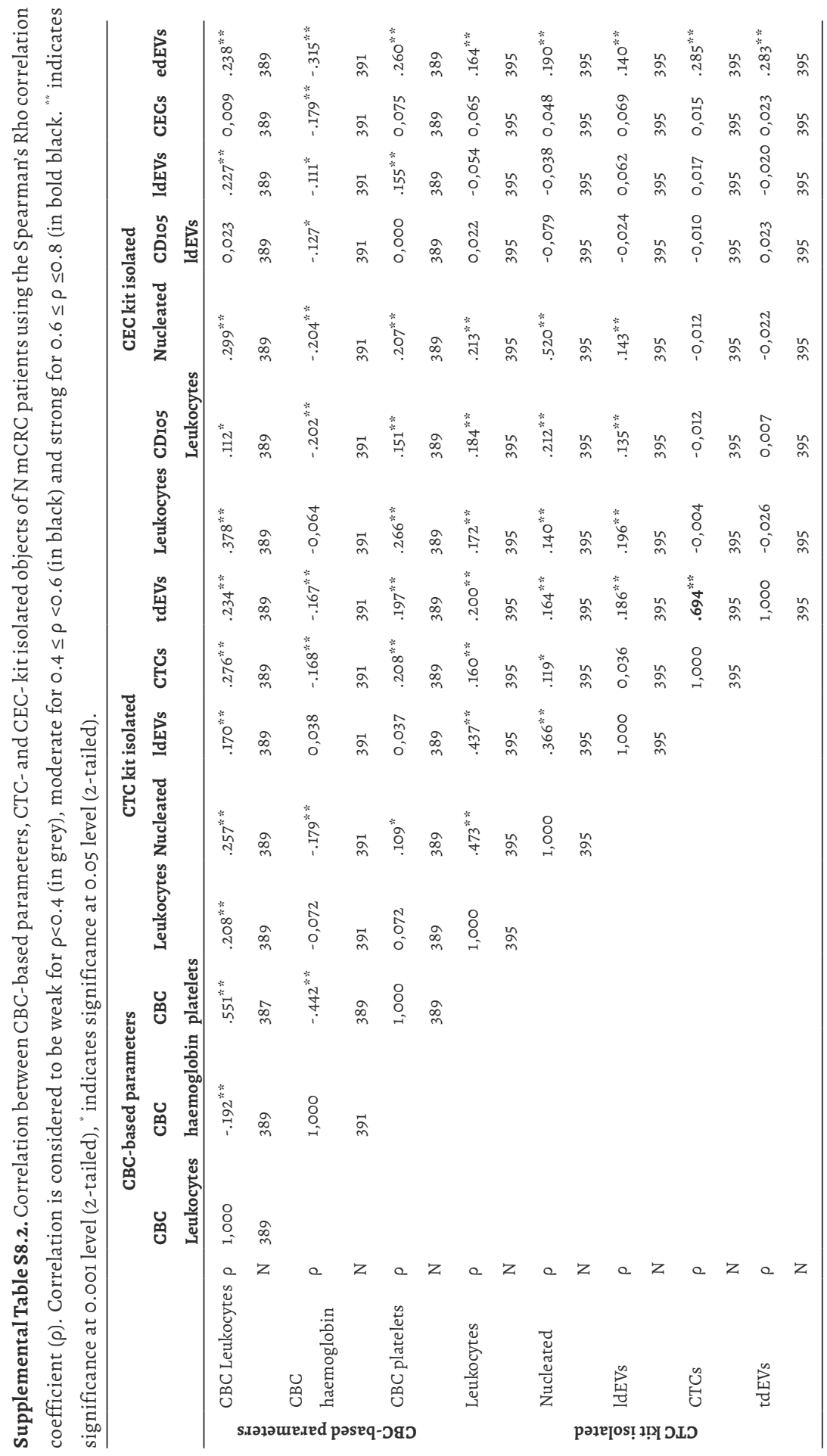




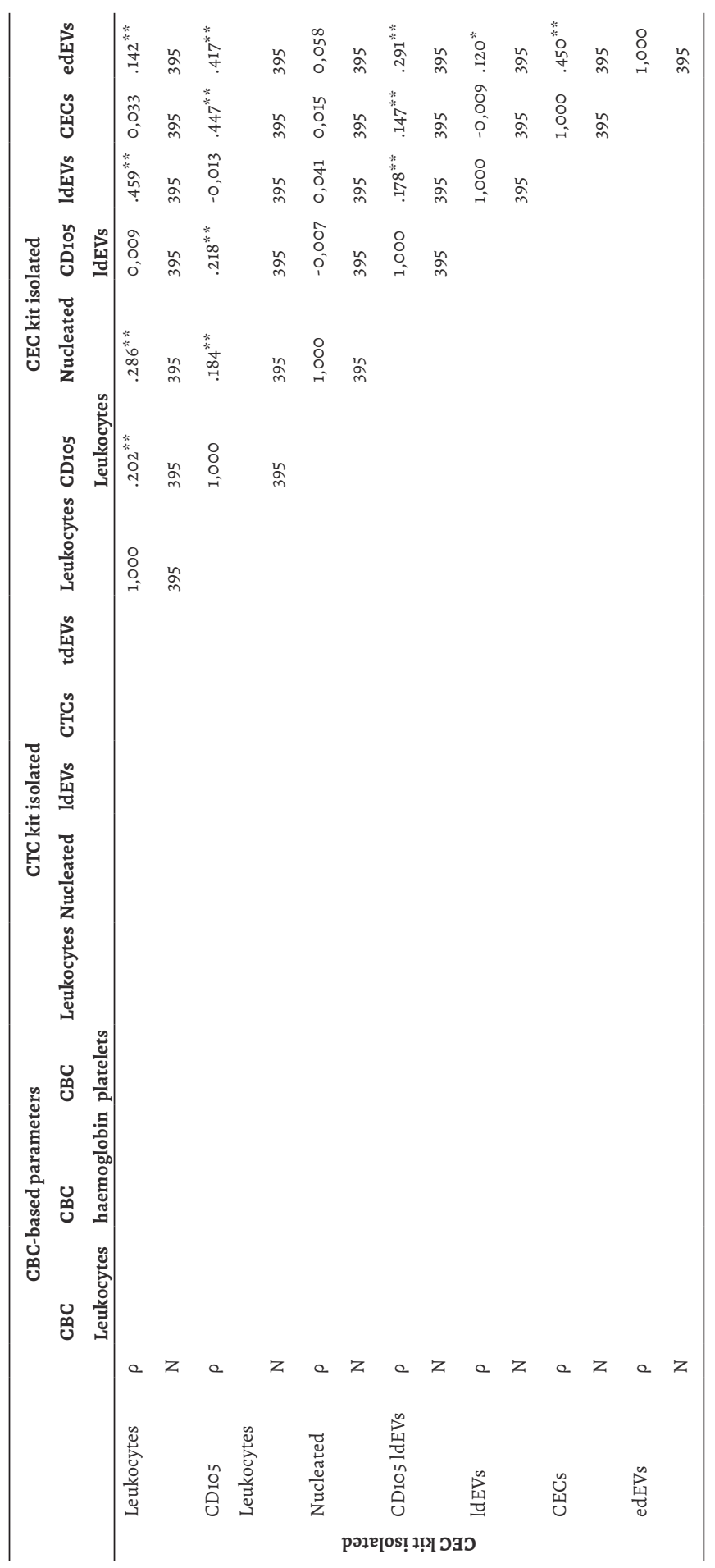




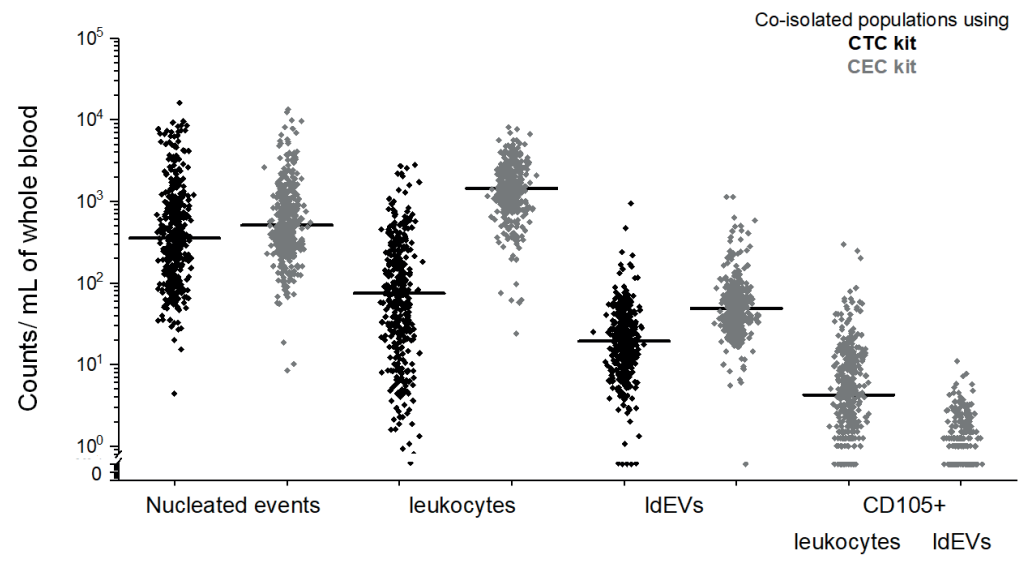

Supplemental Figure S8.1. Frequencies of different populations co-isolated with the CTC (black dots) and CEC kits (grey dots), normalized to $1 \mathrm{~mL}$ of blood for comparison between the kits.

\section{A. Circulating Endothelial Cells} (CECs)

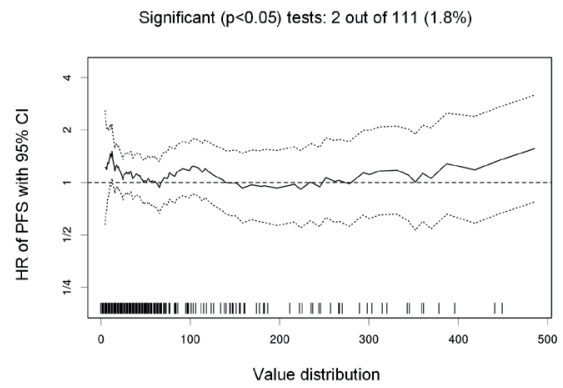

Significant $(p<0.05)$ tests: 0 out of $129(0 \%)$

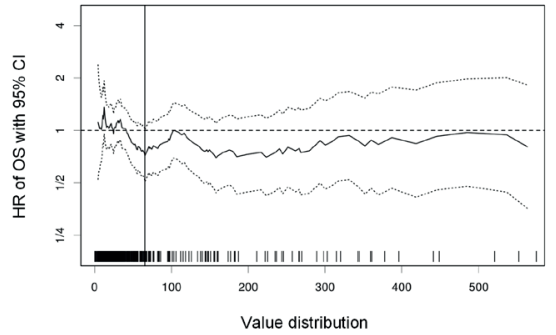

B. endothelium-derived Extracellular Vesicles

(edEVs)

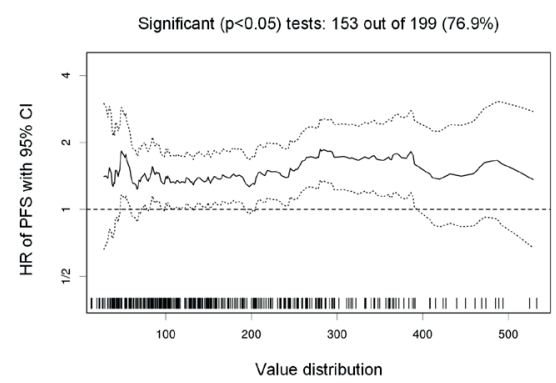

Significant $(p<0.05)$ tests: 157 out of $225(69.8 \%)$

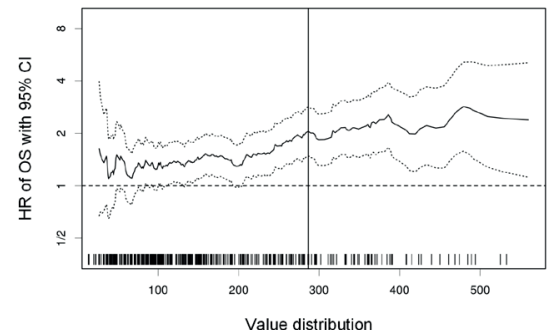

Supplemental Figure S8.2. Cut-off optimization of the baseline values for CECs (Panel A) and edEVs (Panel B) in mCRC patients. For each possible cut-off, CECs and edEVs were correlated with PFS (top) or OS (bottom). The HR including 95\% CI was plotted in dependence of the cutoff. The vertical line indicates the cut-off that results in the most significant correlation with OS. The value distribution of CECs and edEVs is shown as a rug plot at the bottom of the respective figure. 
A. Combined CTCs and edEVs

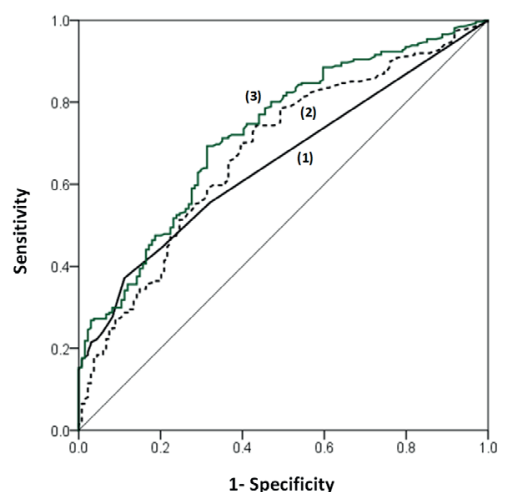

1-Specificity

$\begin{array}{ccc}\text { Variable } & \text { \% AUC } & \text { p-value } \\ \text { CTCs (1) } & 65.2(59.8-70.7) & <0.001 \\ \text { edEVs (2) } & 67.8(62.3-73.3) & <0.001 \\ & & \\ \text { CTCS \& edEVs (3) } & 72.5(67.3-77.6) & <0.001\end{array}$

B. Combined tdEVs and edEVs

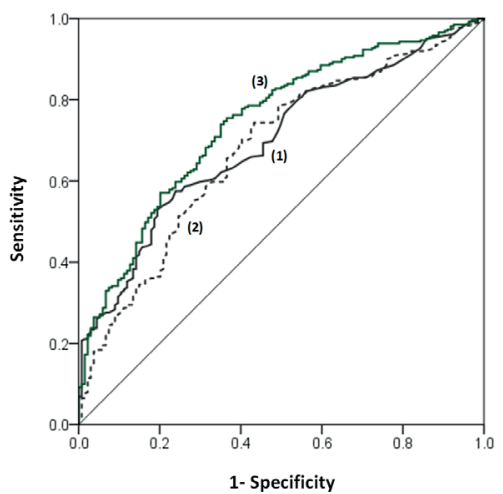

$\begin{array}{ccc}\text { Variable } & \text { \% AUC } & \text { p-value } \\ \text { tdEVs (1) } & 69.6(64.3-74.8) & <0.001 \\ \text { edEVs (2) } & 67.8(62.3-73.3) & <0.001 \\ & & \\ & & <0.001\end{array}$

Supplemental Figure S8.3. Receiver Operating Characteristic (ROC) curves treating survival time dichotomized by the median OS time of the patient cohort as the classification variable. The addition of edEVs to CTCs (Panel A) or tdEVs (Panel B) results in significantly $(\mathrm{p}<0.05)$ higher area under the curve (AUC) compared to solely CTCs or tdEVs. 


\title{
Chapter 9
}

\section{Fusion of functional liposomes with cells: a versatile method for the supramolecular functionalization of cell membranes in vitro}

\author{
Afroditi Nanou*, Mark L. Verheijden", Dodo Wasserberg, Anouk Mentink-Leusink, \\ Pascal Jonkheijm, Leon W.M.M. Terstappen \\ "Both authors contributed equally
}

Manuscript in preparation.

\begin{abstract}
Cellular uptake of liposomes via membrane fusion ensures the molecular cargo delivery to the recipient cells. In the present study, we compared different (des)biotin liposomes in terms of their fusion with cells from cancer cell lines and blood from healthy individuals. DOPC liposomes of similar size distribution containing $20 \mathrm{~mol} \%$

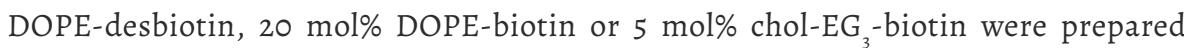
by extrusion. Their mean hydrodynamic diameter was around $100 \mathrm{~nm}$. Leukocyte subpopulations, platelets and different cancer cell lines were incubated with the different liposome systems, stained with fluorophore-tagged streptavidin and their fusion with liposomes was assessed by flow cytometry and immunofluorescence microscopy. The chol-EG 3 -biotin liposomes achieved the highest biotin incorporation into the cell membrane, followed by DOPE-biotin liposomes. DOPE-desbiotin liposomes did not show considerable fusion with any cell type. The highest liposome uptake was found in cancer cells followed by the monocytes indicating a relationship between available cell surface and liposome uptake. Biotin-anchored liposomes can be used to evaluate cellular internalization by membrane fusion. Chol-EG -biotin liposomes performed the best in fusing with the different cell types.
\end{abstract}




\subsection{Introduction}

The liposome field has experienced significant progress in the last decades with particularly promising applications in the biomedical field, where liposomes serve as drug or enzyme carriers ${ }^{1-6}$ as well as vehicles that allow for molecular engineering of the surface of the recipient cells ${ }^{7-9}$. The main feature of liposomes that renders them promising drug carriers, is their relatively higher stability in the bloodstream leading to prolonged circulation, lower cytotoxicity and higher treatment efficiency compared to the free active molecule administration ${ }^{5,10}$. Furthermore, their functionalization with specific ligands (peptides, antibodies and hormones) on their surface can lead to their selective uptake by target cells bearing the respective receptors increasing desired cellular uptake and reducing accumulation, cytotoxicity and side effects in undesired tissues ${ }^{11,12}$.

The effect of liposome size on the interaction with blood cells or cells in target tissue has been studied thoroughly in animal studies, in the case of intravenous administration. Liposomes of 100-500 $\mathrm{nm}$ diameter remain in the core of the bloodstream and follow the blood flow pattern together with the red blood cells with minimal adhesion to the wall ${ }^{13}$, allowing their longer circulation in blood and their interaction with blood cells; hence, they can be used for targeting the liver, spleen and lung, where they will end up eventually for their clearance ${ }^{14,15}$. Larger liposomes with a diameter range between 2-5 $\mu \mathrm{m}$ have the tendency to localize to the vascular wall interacting mainly with leukocytes and endothelial cells composing the vascular wall ${ }^{13}$. Hence, they are good candidates as gene and drug carriers to treat cardiovascular diseases or blood vessel disruption ${ }^{16,17}$. Importantly, the accumulation of encapsulating anti-oncogenic agent liposomes in the tumor site due to the leaky vasculature, described as enhanced permeability and retention (ERP) effect ${ }^{18,19}$, leads to better treatment efficiency compared to the administration of the free agent ${ }^{20,21}$.

Although the biodistribution of liposomes to various tissues after intravenous administration has been well-studied, there are few studies exploring the uptake mechanism of liposomes by different cell types, with two main mechanisms being described namely the active and the passive cellular uptake. The former one is receptor-independent and includes micropinocytosis and incorporation of cholesterol and lipid domains in the plasma membrane, whereas the latter one is receptordependent, including phagocytosis, caveolae- and clathrin-based endocytosis ${ }^{22,23}$. The cellular uptake of liposomes is a critical point in drug delivery, since the endocytosis of liposomes can lead to their transcytosis or degradation by lysosomes rendering cargo delivery to a very inefficient process ${ }^{24,25}$. On the other hand, fusion of liposomes with the cell membrane ensures drug delivery to the cell cytosol ${ }^{26}$.

In the present study, we explored three different liposome systems and compared their fusion with the plasma membrane of different cell types in suspension, namely 
white blood cells and platelets of healthy individuals and cancer cell lines. We used liposomes doped with supramolecular handles, namely phospholipid- or cholesterylanchored biotin or phospholipid- anchored desthiobiotin (desbiotin) to enable the evaluation of their fusion with cells using fluorescently-labeled streptavidin. For this purpose, a desthiobiotin-functionalized lipid (DOPE-desbiotin) and a cholesterylmodified biotin molecule with a short peptide and triethylene glycol spacer (Chol$\mathrm{EG}_{3}$-biotin) were synthesized. The biotin-functionalized lipid (DOPE-biotin) was commercially available.

\subsection{Materials and Methods}

\subsubsection{General methods}

Chemicals were purchased from Sigma-Aldrich Chemie NV (Zwijndrecht, the Netherlands) or from Acros Organics ${ }^{\mathrm{TM}}$ B.V.B.A (Geel, Belgium). Protected amino acids were obtained from MultiSynTech (Witten, Germany) except for Fmoc-lys(biotin)-OH and Fmoc-NH-(PEG) ${ }_{2}-\mathrm{COOH}$ which were obtained from MERCK B.V. (Schiphol-Rijk, the Netherlans). Resin for solid phase peptide synthesis was obtained from Novabiochem ${ }^{\circledR}$. Commercial lipids were obtained from Avanti ${ }^{\circledR}$ Polar Lipids, Inc. (Alabama, U.S.) unless stated otherwise. HEPES buffer contained 0.01 M HEPES and $0.15 \mathrm{M}$ sodium chloride was made using Milli-Q water (MQ Millipore, $18.2 \mathrm{~m} \Omega$ ) and adjusted to $\mathrm{pH} 7.4$ at $25^{\circ} \mathrm{C}$ using sodium hydroxide.

Mass spectra were recorded with a Waters electrospray ionization time of flight mass spectrometer operated in positive ion mode (ESI(+)-ToF, Micromass LCT).

High performance liquid chromatography (HPLC) was performed using a C18 reverse phase column (Waters, Xbridge).

Dynamic light scattering (DLS) analysis was carried out on an Anaspec particle analyser (Nanotrac operating with a Microtrac FLEX Operating Software).

Zeta (ל) potential measurements of the liposomes were performed at $20^{\circ} \mathrm{C}$ with a ZetaSizer instrument (NanoZS, Malvern).

\subsubsection{Synthesis procedures}

DOPE-desbiotin. 1,2-dioleoyl-sn-glycero-3-phosphoethanolamine (DOPE, $24 \mathrm{mg}$, $0.32 \mu \mathrm{mol}$ ) was dissolved in $1 \mathrm{ml}$ chloroform and methanol in a 3:1 ratio. To this solution, $N$-hydroxysuccinimide-desthiobiotin (NHS-dtbiotin, $10 \mathrm{mg}, 0.32 \mu \mathrm{mol}$ ) and $N, N$-diisopropylethylamine (DIPEA, $6.7 \mu \mathrm{l}, 0.39 \mu \mathrm{mol}$ ) were added under argon and stirred overnight at room temperature. The solution was then diluted with chloroform 
and extracted with $5 \%$ aqueous ammonia. The aqueous layer was extracted again with chloroform and the combined layers were concentrated. Further purification using silica gel chromatography ( $1 \%$ aqueous ammonia, $10 \%$ methanol in dichloromethane) gave, after freeze drying, pure product as white powder. Yield: $8 \mathrm{mg}(27 \%) ;{ }^{1} \mathrm{H}-\mathrm{NMR}$ (400 MHz, $\mathrm{CDCl}_{3}$, Appendix 9.A): $\delta=0.81\left(\mathrm{t}, 6 \mathrm{H}, \mathrm{CH}_{3} \mathrm{CH}_{2}\right.$ ), $1.10\left(\mathrm{~d}, 3 \mathrm{H}, \mathrm{CH}_{3} \mathrm{CH}\right) 1.2-1.82$ $\left(\mathrm{m}, 52 \mathrm{H}, \mathrm{CH}_{3} \mathrm{CH}_{2} \mathrm{CH}_{2}+\mathrm{CH}_{2} \mathrm{CH}_{2} \mathrm{CH}_{2}+\mathrm{NHCHCH}_{2}\right) 1.93\left(\mathrm{~m}, 8 \mathrm{H}, \mathrm{CHCHCH}_{2}\right) 2.24(\mathrm{~m}, 6 \mathrm{H}$, $\left.\mathrm{OCOCH}_{2}\right) 3.42-3.75\left(\mathrm{~m}, 4 \mathrm{H}, \mathrm{NHCH}_{2} \mathrm{CH}_{2}+\mathrm{NHCH}\right) 3.92-4.32\left(\mathrm{~m}, 7 \mathrm{H}, \mathrm{POCH}_{2}+\mathrm{CHCH}_{2} \mathrm{OP}\right.$ $+\mathrm{CHCH}_{2} \mathrm{OP}+\mathrm{OCOCH}_{2} \mathrm{CH}$ ) 5.16-5.27 (m, 4H, CHCH), 7.6 (bs, $1 \mathrm{H}, \mathrm{CH}_{2} \mathrm{NHCO}$ ), 8.21 (bs, 2H, CHNHCO). ESI(+)-ToF MS (Appendix 9.A): $[\mathrm{M}+2 \mathrm{H}]^{+}=940.6486$ (calc. 940.6750), $[\mathrm{M}+\mathrm{H}+\mathrm{Na}]^{+}=962.6315$ (calc. 962.6569), $[\mathrm{M}+2 \mathrm{Na}]^{+}=984.6144$ (calc. 984.6388).

Chol-EG -biotin. Chol-EG -biotin was synthesized using automated solid phase peptide synthesis (Syro II, MultiSynTech) following standard fluorenylmethyloxycarbonyl group (Fmoc) - based procedures on a Rink amide 4-Methylbenzhydrylamine (MBHA) resin (100-200 mesh, loading $0.52 \mathrm{mmol} / \mathrm{g}$ ) using hexafluorophosphate benzotriazole tetramethyl uronium (HBTU), Hydroxybenzotriazole (HOBt) and DIPEA. The oligo ethylene glycol linker was introduced using Fmoc-NH-(PEG) $-\mathrm{COOH}$ (MERCK) and biotin was introduced using Fmoc-lys(biotin)OH (MERCK). Cholesterol hemisuccinate (Chol) was coupled manually in the last step using benzotriazol-1-yl-oxytripyrrolidinophosphonium hexafluorophosphate (PyBOP), HOBt and DIPEA. Cleavage and deprotection of the peptide were performed by adding to the resin a solution of $2.5 \mathrm{vol} \%$ water and 2.5 vol\% triisopropylsilane in trifluoroacetic acid (TFA) and stirring for $4 \mathrm{~h}$. Purification was performed by repeated (3x) precipitation in cold diethyl ether and subsequent preparative HPLC using an XBridge C18 reverse phase column. The HPLC program consisted of $10 \mathrm{~min}$ flow with $1 \%$ acetonitrile in water and then a $40 \mathrm{~min}$ gradient up to $100 \%$ acetonitrile, always in presence of $0.1 \%$ TFA and using a $7 \mathrm{ml} / \mathrm{min}$ flow rate. The functionalized peptide was characterized using ESI(+)-ToF.

\subsubsection{Fabrication of liposomes}

1,2-Dioleoyl-sn-glycero-3-phosphocholine (DOPC), 1,2-dioleoyl-sn-glycero-3phosphoethanolamine-N-(cap biotinyl) (DOPE-biotin) as well as the synthesized lipids DOPE-desbiotin and chol-EG -biotin were stored in chloroform at $-20^{\circ} \mathrm{C}$. Dissolved lipids were mixed in desired molar ratios before use and dried under a flow of nitrogen in a glass vial in order to create a film of lipid material at the glass wall. This film was further dried under vacuum for at least $1 \mathrm{~h}$ and subsequently, hydrated by vortexing with Milli-Q water to form multilamellar liposomes at $1 \mathrm{mg} / \mathrm{mL}$. The lipid suspension was extruded 11 times through a polycarbonate membrane (Whatman) with $100 \mathrm{~nm}$ pore size, resulting in large unilamellar liposomes (LUVs) that were stored in the fridge and used within two weeks. 


\subsubsection{Biological samples and cells from cell lines}

Breast cancer cell lines MCF-7 and SKBR3 and melanoma cancer cell line A2058 were obtained from the American Type Culture Collection ATCC (Rockville, MD). The cell lines were cultured at $37{ }^{\circ} \mathrm{C}$ and $5 \% \mathrm{CO}_{2}$ in Roswell Park Memorial Institute (RPMI) 1640 with L-glutamine (Thermo Fischer Scientific, cat \# 11875) medium, supplemented with $10 \% \mathrm{v} / \mathrm{v}$ fetal bovine serum (Lonza, lot \# BCBT0730), 10 units/mL penicillin and $10 \mu \mathrm{g} / \mathrm{ml}$ streptomycin (Lonza, lot \# 7MBO20). The medium was refreshed every two to three days. The initial cell density was $10-15,000$ cells $/ \mathrm{cm}^{2}$, as recommended by ATCC and cells were trypsinized and harvested for the conduction of the experiments or passaged when they reached $80 \%$ confluence.

\subsubsection{Blood samples}

Blood samples from anonymous healthy donors were collected in ethylenediaminetetraacetic acid (EDTA) blood draw tubes from the Experimental Centre for Technical Medicine (ECTM) donor service (University of Twente, Enschede, The Netherlands). The donors provided informed consent prior to blood donation, in accordance to the study protocol approved by the METC Twente ethics committee.

\subsubsection{Leukocyte collection}

Two different ways were used for the collection of white blood cells from whole blood, namely density gradient separation using Ficoll-Paque Plus (GE Healthcare) to obtain only the mononuclear cell and red blood lysis using ammonium chloride to obtain all nucleated cells. The protocol suggested from the manufacturer of the Ficoll-Paque Plus was followed for the isolation of mononuclear cells from whole blood. In case of the red blood cell lysis, blood was diluted $20 \mathrm{x}$ in filtered with $0.2 \mu \mathrm{m}$ freshly prepared ammonium chloride ( $155 \mathrm{mM}$ ammonium chloride, $10 \mathrm{mM}$ potassium bicarbonate, 0.1 $\mathrm{mM}$ ethylene dinitrilo tetraacetic acid disodium salt, $\mathrm{pH}=7.25$ ) and incubated in ice for 20 min until full red blood cell lysis. White blood cells were centrifuged at $300 x \mathrm{~g}$ for $10 \mathrm{~min}$, the pellet was washed twice in filtered $1 \% \mathrm{w} / \mathrm{v}$ bovine serum albumin (BSA) (Sigma Aldrich) in phosphate buffer solution (PBS) (Sigma Aldrich) and cells were re-suspended in $1 \% \mathrm{w} / \mathrm{v}$ BSA in PBS of the same volume as the whole blood volume used. The two protocols were compared in regards to their effect on the fusion of the differently isolated cells with the liposomes and no differences were observed.

\subsubsection{Collection of platelets}

EDTA blood was centrifuged at $300 \mathrm{xg}$ for $10 \mathrm{~min}$ without brake and platelet-rich plasma was collected without disturbing the buffy coat. Platelet-rich plasma was centrifuged at $1730 x g$ for $7 \mathrm{~min}$, the supernatant was discarded and the pellet containing most of the platelets was collected and re-suspended in $0.2 \mu \mathrm{m}$ filtered $1 \% \mathrm{w} / \mathrm{v}$ BSA in PBS for further use. 


\subsubsection{Fusion of (des)biotinylated liposomes with cells from cancer cell lines, white blood cells and platelets}

Different cell types were investigated regarding their fusion with the different liposome systems. In case of cancer cell lines (MCF7, SKBR3 and A2058) and white blood cells, 8 aliquots of $150,000-200,000$ cells were pipetted in flow cytometry tubes. In the case of platelets, 500,000 cells were pipetted. Cells were centrifuged at the correct centrifugation force $(500 \mathrm{xg}$ for $5 \mathrm{~min}$ in case of cell lines and white blood cells and $1730 \mathrm{xg}$ for $7 \mathrm{~min}$ in case of platelets) and their pellets were re-suspended in $180 \mu \mathrm{l}$ of $0.2 \mu \mathrm{m}$ filtered $1 \% \mathrm{w} / \mathrm{v}$ BSA in PBS. $20 \mu \mathrm{l}$ of $1 \mathrm{mg} / \mathrm{ml}$ liposomes were added to the correct samples to a final liposome concentration of $0.1 \mathrm{mg} / \mathrm{ml}$ following $\mathrm{I} \mathrm{h}$ incubation at room temperature. Cells were washed once with $1 \% \mathrm{w} / \mathrm{v}$ BSA in PBS and incubated for $30 \mathrm{~min}$ at room temperature with staining solution including $0.25 \mu \mathrm{g} / \mathrm{ml}$ phycoerythrin-conjugated antibody recognizing the leukocyte-specific cluster of differentiation 45 (CD45-PE) (HI30 clone, BioLegend, California, U.S.) or $0.25 \mu \mathrm{g} / \mathrm{ml}$ phycoerythrin-conjugated antibody recognizing the

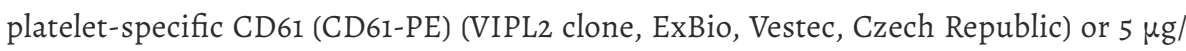
$\mathrm{ml}$ phycoerythrin-conjugated antibody recognizing the epithelial cell adhesion molecule (EpCAM-PE) (HI30 clone, BioLegend), $4 \mu \mathrm{g} / \mathrm{ml}$ allophycocyanin-conjugated streptavidin (SAV-APC) (Southern Biotech, Birmingham, U.S.) or 1onM SAV-PE (Sigma)). One more washing step was followed and finally, cells were labeled with $4 \mu \mathrm{g} / \mathrm{ml}$ of the nuclear dye Hoechst 33342 (Life Technologies) for $10 \mathrm{~min}$ at $37^{\circ} \mathrm{C}$ and kept afterwards in ice until measurement with flow cytometry.

\subsubsection{Flow cytometry}

Flow cytometry was carried out on a BD Biosciences flow cytometer (FACSAria ${ }^{\mathrm{TM}}$ II) equipped with a $375 \mathrm{~nm}$, a $488 \mathrm{~nm}$ and a $633 \mathrm{~nm}$ laser. For Hoechst detection, the $375 \mathrm{~nm}$ laser was used for excitation and a $450 / 40 \mathrm{~nm}$ emission filter; for PE detection, a 488 $\mathrm{nm}$ laser was used for excitation and a 585/42 nm emission filter; for APC detection, a $633 \mathrm{~nm}$ laser was used for excitation and a $660 / 20 \mathrm{~nm}$ emission filter.

\subsubsection{Fluorescence microscopy}

For the acquisition of fluorescence microscopy images, a Nikon E400 microscope equipped with a light-emitting diode (LED) source (SOLA light engine, Lumencore ${ }^{\circ}$ ), a charged-coupled device (CCD) camera (Orca flash 4.0 LT, C11440, Hamamatsu, Almere, The Netherlands), X-Y-Z stage and fluorescence filter cubes (DAPI, PE and APC filter sets for the detection of Hoechst, CD61 /CD45 / EpCAM and streptavidin, respectively). In microscopy images displayed in this work, contrast and brightness were adapted for clarity using ImageJ 1.51k (National Institutes of Health, USA). 


\subsection{Results}

\subsubsection{Size distribution of liposome systems}

Zwitterionic DOPC liposomes containing up to 20 mol\% DOPE-biotin, 20\% DOPEdesbiotin or $5 \mathrm{~mol} \%$ Chol-EG -biotin were formed and their schematic presentation is illustrated in Figure 9.1.

All 3 liposome systems were nearly monodisperse with a hydrodynamic diameter of approximately $100 \mathrm{~nm}$ and were stable for more than one month, as supported by the DLS measurements shown in Figure 9.2. Note that DOPC liposomes with $5 \mathrm{~mol} \% \mathrm{Chol}$ $\mathrm{EG}_{3}$-biotin were used since $20 \mathrm{~mol} \% \mathrm{Chol}-\mathrm{EG}_{3}$-biotin liposomes were polydisperse and not stable. The use of $20 \mathrm{~mol} \%$ of the other biotin and desbiotin ligands was maintained in order to achieve the highest possible labeling efficiency.

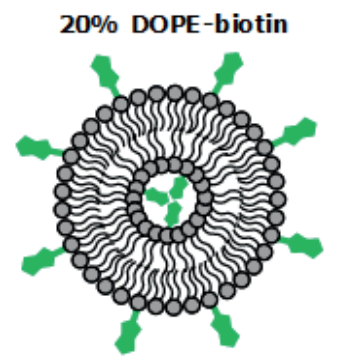

$20 \%$ DOPE-desbiotin

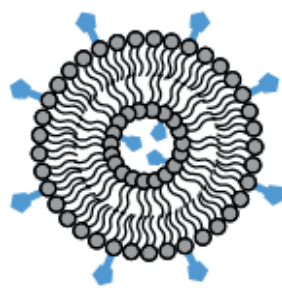

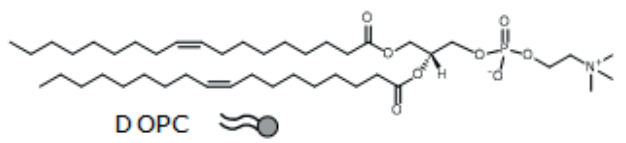
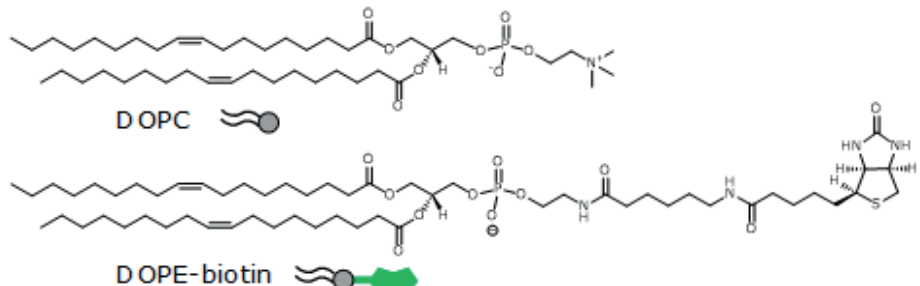

D OPE-biotin

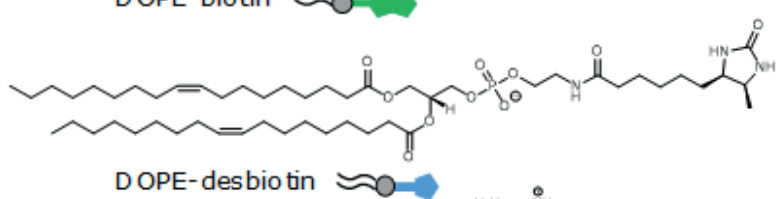

DOPE-desbiotin

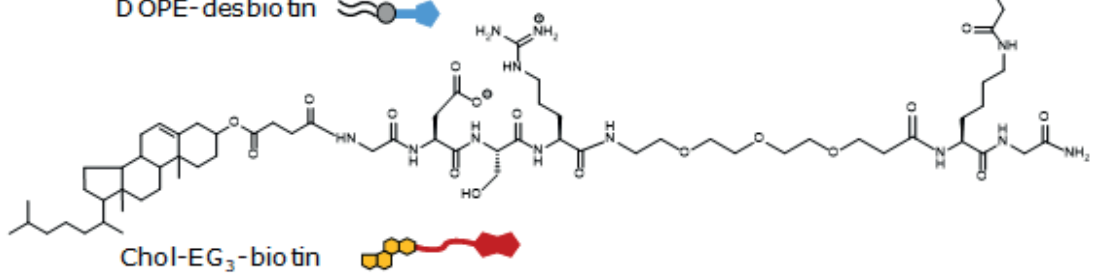

Figure 9.1. Supra-molecularly functionalized liposome systems used. Schematic presentation of the liposome-based systems used for the supramolecular cell functionalization. The structures of the main molecules are also shown. 


\subsubsection{Definition of cell populations used by flow cytometry}

Platelets and the white blood cell (WBC) subpopulations, lymphocytes, granulocytes and monocytes of healthy individuals and cells from the breast cancer cell line MCF7 were incubated with liposomes and analyzed by flow cytometry. Cells were identified by their light scattering properties as well as the presence of nucleus (stained by Hoechst) and the expression of cell surface receptors characteristic for the target cells (CD61 for platelets, CD45 for white blood cells and EpCAM for cells from the carcinoma cell lines) by the respective PE-labeled antibodies, as shown in Figure 9.3. In the same figure, the histogram of APC intensity is shown to illustrate the non-specific binding of SAV-APC after incubation with cells but without pre-incubation with liposomes.

\subsubsection{Cellular uptake of liposome systems by different cell types}

To compare the three different (des)biotin liposomes in terms of their ability to fuse and functionally display their (des)biotin ligands, cells were labeled with SAV-APC after their incubation with liposomes and evaluated by flow cytometry and fluorescence microscopy. From the SAV histograms of a representative experiment (Figure 9.4), the averaged results of all experiments (Figure 9.5 and Table 9.1), it follows that large differences exist in the labeling efficiency depending on both the cell type and the liposome type used.

A.

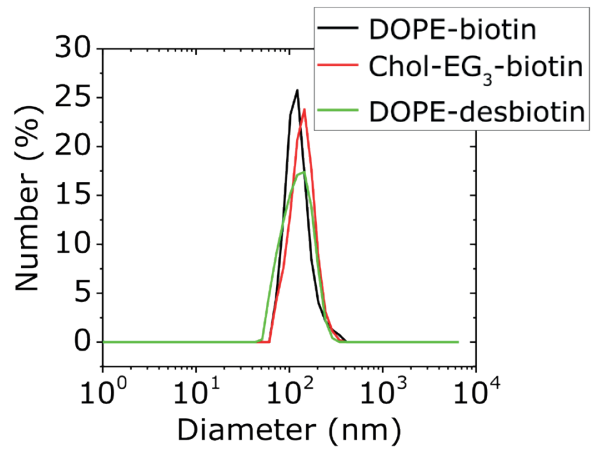

B.

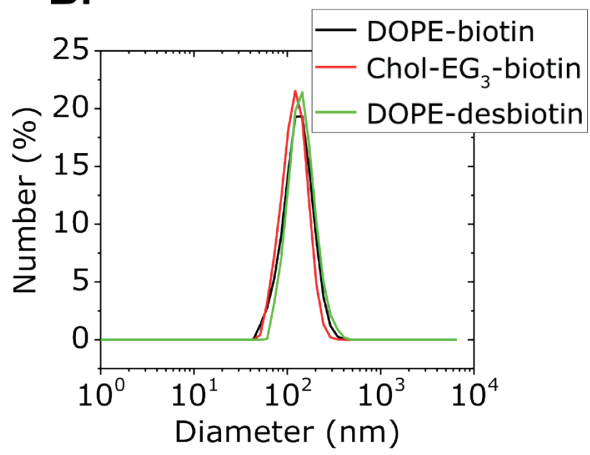

Figure 9.2. Liposome size distributions. Overlay of size distribution of DOPC liposomes with 20 mol\% DOPE-biotin, 20 mol\% DOPE-desbiotin or 5 mol\% Chol-EG -biotin (A) directly after extrusion and (B) after $>1$ month storage at $4^{\circ} \mathrm{C}$ as measured by DLS. 

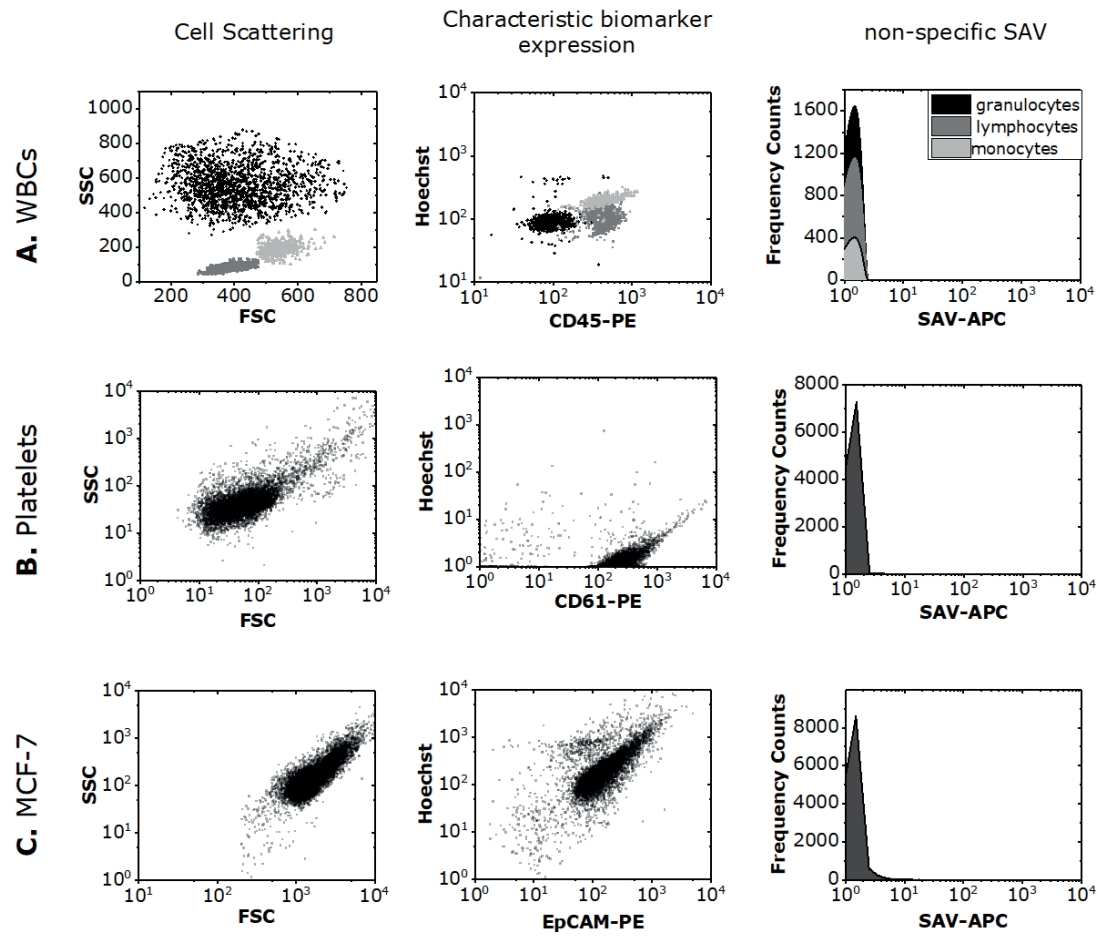

Figure 9.3. Flow cytometric definition of cell populations. Left column: Forward and side light scattering of white blood cells (Panel A), platelets (Panel B) and MCF-7 cells (Panel C). Middle column: Hoechst (DNA) staining versus CD45-PE antibody staining (Panel A), CD61-PE antibody staining (Panel B) and EpCAM-PE antibody staining (Panel C). Right column: negative control of non-specific binding of SAVAPC to all cell types.

The "labeling efficiency" is here defined by the amount of SAV-APC binding, which will be determined by the cellular uptake of the (des)biotin liposomes by fusion, the functional display of (des)biotin at the cell surface and the binding of SAV to (des)biotin anchors.

Incubation with DOPE-desbiotin liposomes did not result in significant SAV labeling for any of the cell types; only monocytes and MCF7 cells showed some increase in the APC fluorescence signal. On the contrary, all cell types could be functionalized with biotin, indicating their fusion with the chol-EG -biotin and DOPE-biotin liposome systems. Between the two biotin-anchored liposome systems, the Chol-EG-biotin liposomes resulted in the highest labeling efficiency for all tested cell types. Both Chol-EG ${ }_{3}$-biotin and DOPE-biotin showed the highest labeling efficiency in MCF-7 cells followed by the monocytes, granulocytes, lymphocytes and platelets. This relation may well be explained by the differences in available cell surface as MCF-7 cells are the largest $(\sim 16 \mu \mathrm{m})$ followed by monocytes $(\sim 12-20 \mu \mathrm{m})$, granulocytes $(\sim 10-18 \mu \mathrm{m})$, lymphocytes $(\sim 10-18 \mu \mathrm{m})$ and platelets $(\sim 1-3 \mu \mathrm{m})^{27,28}$. 


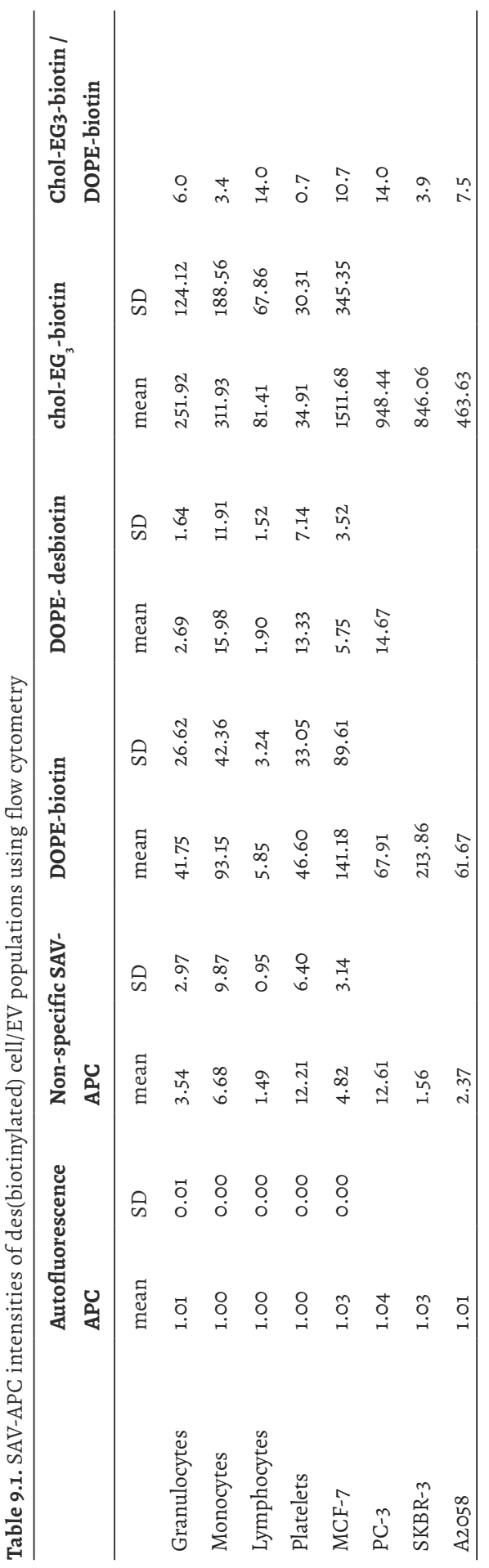



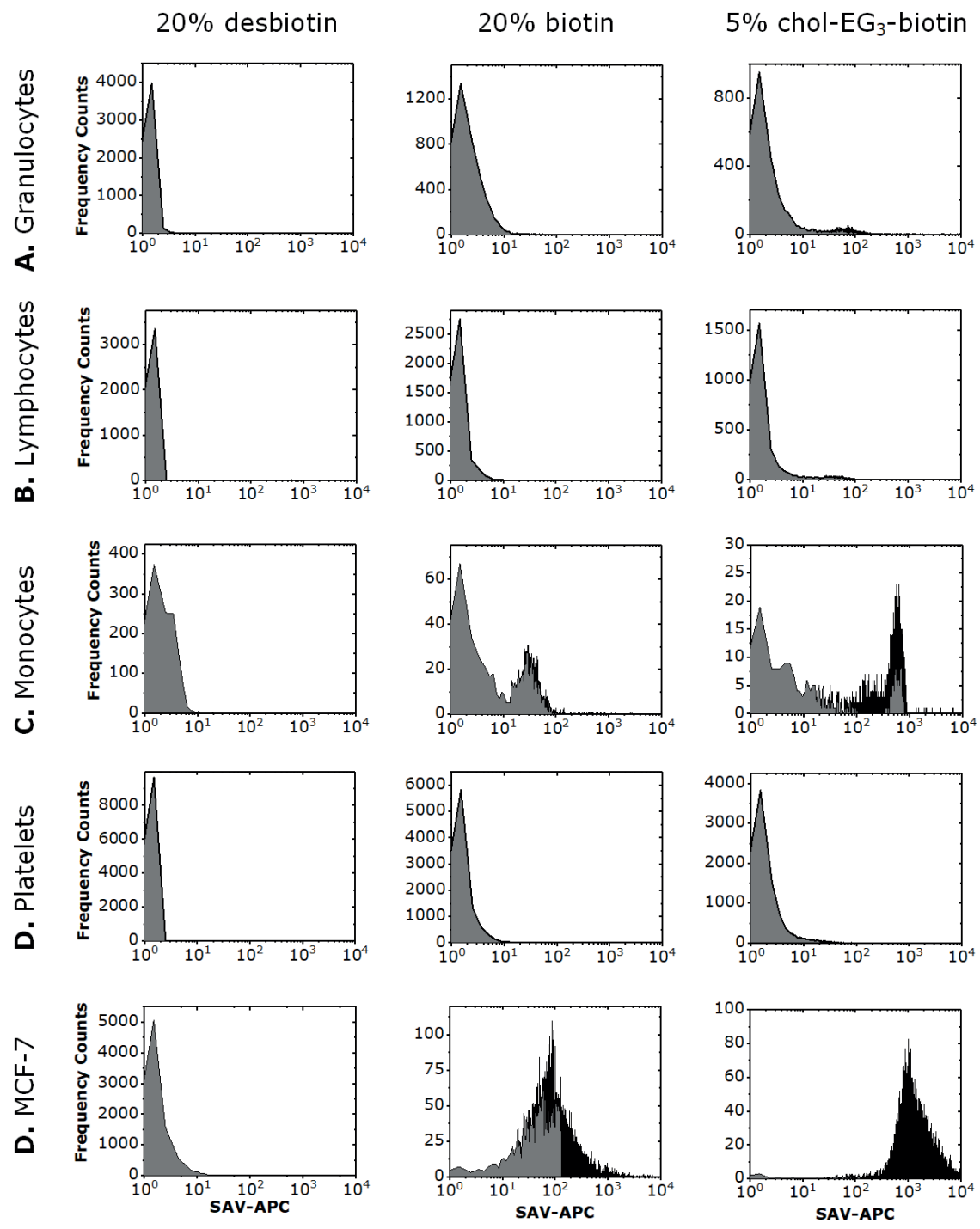

Figure 9.4. SAV-APC histograms showing the (des)biotin tagging of different cell types. Distribution of the SAV-APC fluorescence intensity of granulocytes (Panel A), lymphocytes (Panel B), monocytes (Panel C), platelets (Panel D) and MCF-7 cells (Panel E) after incubation with (left column) DOPEdesbiotin liposomes, (middle column) DOPE-biotin liposomes and (right column) Chol-EG -biotin liposomes. Each histogram corresponds to 1,000-10,000 events. 


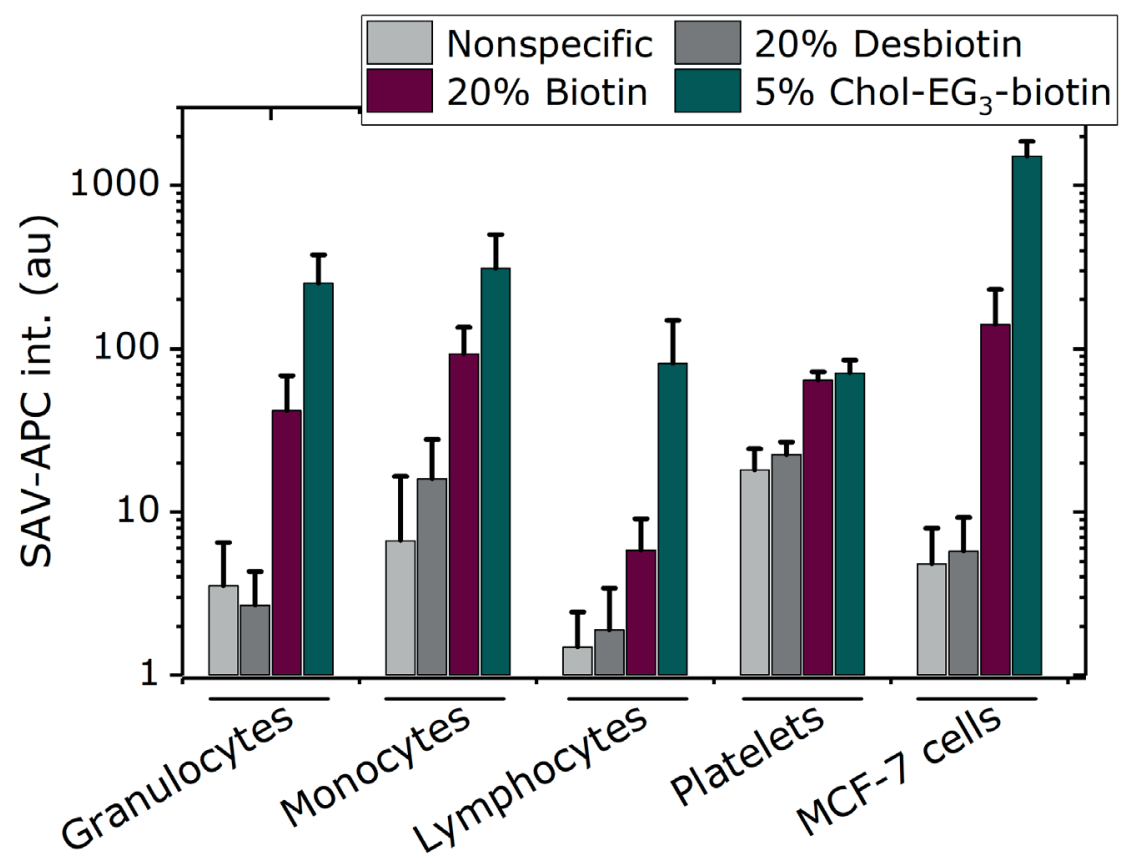

Figure 9.5. Overview of (des)biotin labeling efficiency. Averages of SAV-APC fluorescence intensities of 2 - 6 biological replicates of granulocytes, monocytes, lymphocytes, platelets and MCF-7 cells after incubation with liposomes displaying DOPE (des)biotin or Chol-EG -biotin. For each experiment, at least 1000-10,000 cells were measured. Error bars indicate standard deviations based on at least 2 measurements.

To visualize the supramolecular functionalization of cells with des(biotin), white blood cells were labeled with SAV-APC and imaged by fluorescence microscopy (Figure 9.6). SAV-APC intensity varied based on the liposome systems used with the chol-EG biotin showing the highest labeling efficiency and DOPE-desbiotin liposome resulting in almost null fusion with the cells. The differential SAV-APC intensity based on the various white blood cell subpopulations (as indicated by their CD45 expression levels) can be clearly visualized with most cells fusing with the (des)biotin DOPE liposomes being $\mathrm{CD} 45+$. Interestingly, in case of chol-EG -biotin liposomes, all cells are SAV+ regardless their $\mathrm{CD} 45$ expression implying a less selective fusion mechanism of these liposomes compared to (des)biotin DOPE ones. Moreover, smaller CD45- and Hoechst- objects, were also detected in the APC channel. These objects are most likely platelets or large Extracellular Vesicles. It is very unlikely that this signal comes from free liposomes, since all liposome systems had a similar size distribution and the same washing steps were included in the different conditions before cell imaging. 

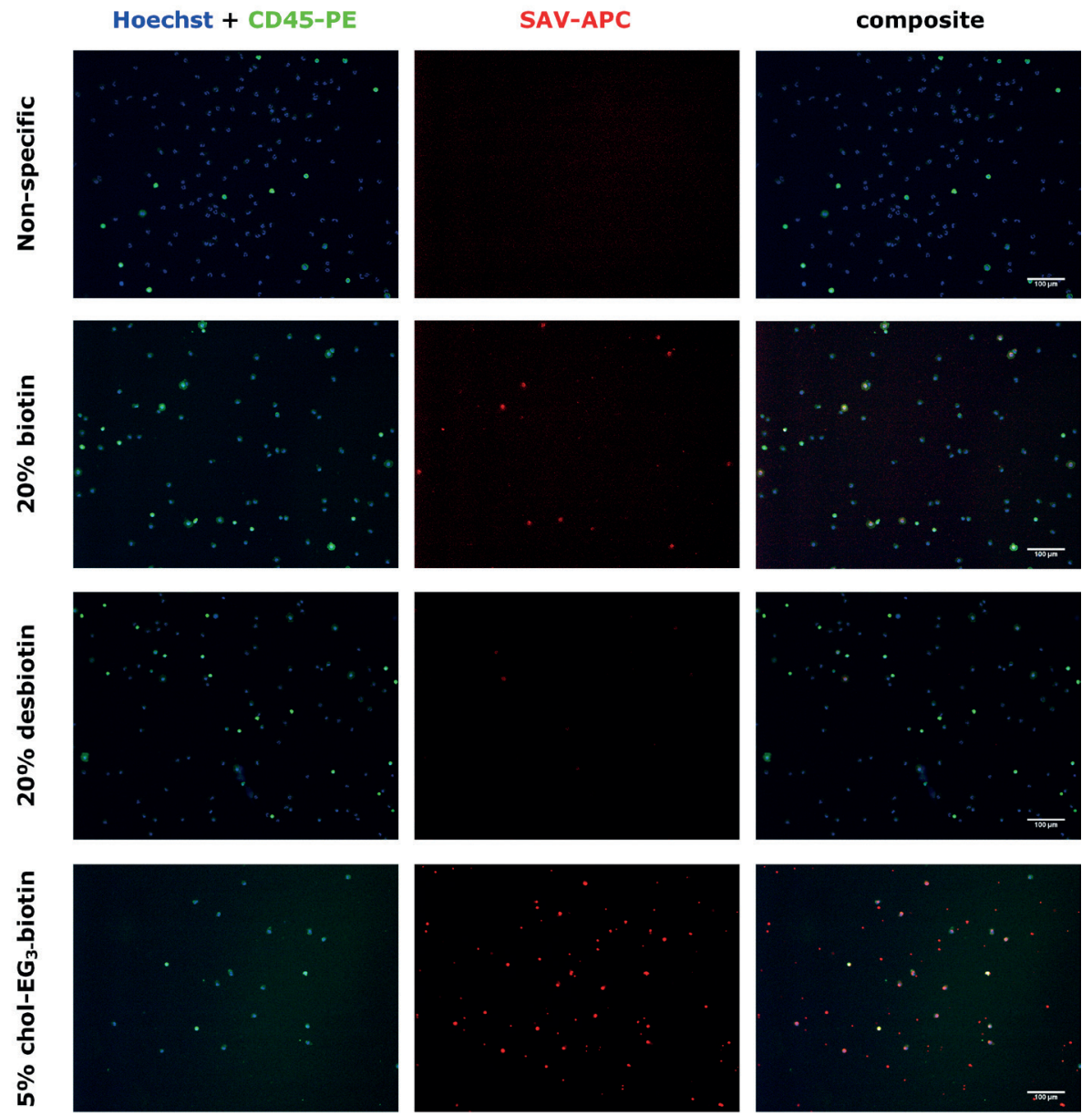

Figure 9.6. Immunofluorescence images of white blood cell subpopulations after fusion with the investigated liposome systems. Scale bars in the composite images represent $100 \mu \mathrm{m}$.

\subsubsection{Effect of cell size and surface morphology on fusion with liposomes}

To further investigate the effect of available cell surface on the fusion with the biotinanchored liposomes, two additional cell lines with different size and tissue of origin were evaluated and compared, namely cells from the melanoma cancer cell line A2058 (mean diameter of $16.3 \mu \mathrm{m}$ ) and cells from the breast cancer cell line SKBR3 (mean diameter of $17.7 \mu \mathrm{m}$ ). The summarized results of the two populations can be found in Appendix 9.D (Panel A shows the light scattering of cells, Panel B the SAV-APC intensity histograms after incubation with DOPE-biotin and Panel C the SAV-APC intensity histograms after incubation with chol-EG -biotin). It is very clear from that figure that each liposome system fused with higher efficiency with the larger SKBR3 
cells compared to the A2058 cells. Moreover, the chol-EG -biotin liposome system performs again better than DOPE-biotin for both cell lines in consistency with our previous results on different cell types. It is worth mentioning that the relative SAV-

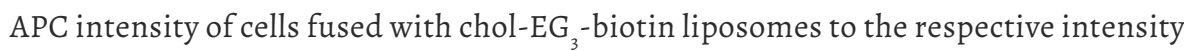
of cells fused with DOPE-biotin liposomes is not the same for all cell types (last column, Table 9.1) implying that an additional factor of cell membrane influences the fusion of the different liposomes with the cells. That observation can be attributed to several factors, such as the membrane biochemical composition and the membrane morphology (granulocytes have smoother surfaces compared to monocytes that have surface ruffles and blebs increasing the available surface).

\subsection{Discussion}

Knowledge of the cellular uptake mechanism of liposomes is very important in the drug delivery field, because endocytosis does not ensure cargo delivery due to the endosomal pathway, whereas fusion with the plasma membrane does. In the former case, the design of more sophisticated systems is required for the active release of the encapsulated molecular cargo, such as activation of a tissue-specific enzyme ${ }^{29}$ or the application of external heat source ${ }^{30,31}$. Regardless the importance of the underlying cellular mechanism, most assays evaluate the liposome incorporation into cells based on their radio- or fluorescent labelling without clear discrimination between the cellular uptake mechanism. The loading of micelles with two fluorescence resonance energy transfer (FRET) probes allows for the assessment of their cellular uptake by either endocytosis or fusion ${ }^{32}$. An alternative assay with the advantage of using a single fluorescent marker, namely the BODIPY tracer, has been also suggested. That is based on the two emission peaks at high concentrations (endocytosis) and only one in lower concentrations (fusion) ${ }^{33}$.

In the present study, we suggest the presence of (des)biotin supramolecular handles on liposome membrane to evaluate their fusion with the recipient cells by labeling the latter ones with fluorophore-tagged streptavidin and measuring them by flow cytometry. Towards that direction, desbiotin (Appendix 9.A) and chol-EG -biotin (Appendix 9.B) were synthesized and used for the formation of $20 \mathrm{~mol} \%$ DOPE-desbiotin DOPC and 5 mol\% chol-EG -biotin DOPC liposome systems respectively. The linker in case of chol$\mathrm{EG}_{3}$-biotin was chosen because of its hydrophilicity that aids the functional display of the cholesterol at the lipid membrane ${ }^{34} .20$ mol\% DOPE-biotin DOPC was the third liposome system formed from commercially available phospholipids. All three liposome systems had a similar hydrodynamic diameter and were tested in terms of their fusion with different cell types, namely white blood cell types, platelets and cancer cell lines.

There were noticeable differences in (des)biotin cell labeling using the different

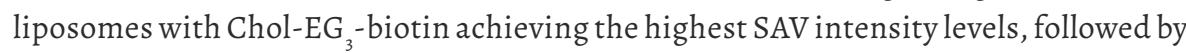


DOPE-biotin, whereas DOPE-desbiotin did not show positivity in SAV signal. Taking into account that chol-EG - biotin liposomes had a 4-fold lower molecular percentage of biotin compared to DOPE-biotin liposomes ( $5 \mathrm{~mol} \%$ versus $20 \mathrm{~mol} \%$ respectively), the conclusion that the fusion with cells is 4 times higher than what we report here would be fair. In both biotin liposome systems, the highest amount of biotin was incorporated in the membrane of MCF7 cells, followed by monocytes. Our findings that the highest liposome incorporation in human leukocytes occurs in monocytes, followed by granulocytes with almost null uptake by lymphocytes are consistent with the previously reported findings of Kuhn et al. ${ }^{35}$, who also suggested a recipient cell surface-dependent fusion of liposomes. However, the observation that the relative SAV signal of the two different biotin liposomes is not constant among the different cell types (Table 9.1) suggests that additional factors play a role in their fusion with liposomes, such as the biochemical composition of the cellular membrane, the charge and the presence of cholesterol molecules in liposomes.

The consistently more efficient biotin labeling of cells using Chol-EG -biotin can be attributed mainly to the presence of cholesterol, which is known to play a dominant/ critical role in the fusion of membranes ${ }^{36}$. More specifically, the relatively small polar (hydrophilic) head group compared to the disproportionately large apolar (hydrophobic) part of cholesterol leads to the formation of concave structures and a negative curvature of cholesterol-containing membranes ${ }^{37,38}$. That effect of cholesterol has been accountable for reducing the required energy for the formation of structural lipid intermediates required for membrane fusion and the stabilization of fusion pores ${ }^{37,39,40}$. The findings of Lee et al. showing that increasing cholesterol (up to 20 mol\%) results in increasing fusion further support the aforementioned hypothesis ${ }^{41}$. The role of cholesterol in biological membrane fusion has been further confirmed by cholesterol depletion in viral membranes hindering virus entry into the target cells ${ }^{42}$.

Another reason explaining the higher SAV signal could be again the longer linker between biotin and cholesteryl anchor (Figure 9.1) making biotin more accessible for SAV binding. Finally, Chol-EG -biotin is overall neutral at physiological $\mathrm{pH}$, while DOPE-(des)biotin displays a negative charge. The resulting negatively charged liposomes might bind and fuse less efficiently with cells ${ }^{43,44}$. Nonetheless, preliminary data of two liposome systems with the same molecular desbiotin composition (100 mol\%), but different charge (Appendix 9.C) supports that the positively charged liposomes are more efficiently taken up by cells by endocytosis compared to the negatively charged ones; on the other hand, negatively charged liposomes are fused more efficiently with cells than positively charged liposomes. From the same data, it is clear that the main cellular uptake mechanism of cholesterol-free liposomes is endocytosis instead of fusion. Further research is recommended to investigate the main mechanism of cholesterol-containing liposomes. 
DOPE-desbiotin and DOPE-biotin liposomes had similar size distribution, charge and molecular composition ( $20 \mathrm{~mol} \%$ ); hence, their fusion efficiency with the different cell types should be the same. The observation of almost null SAV positivity after incubation with DOPE-desbiotin liposomes suggests that the specific liposome system is not suitable for the evaluation of fusion with cells. Several reasons can explain the difference in SAV signal between DOPE-biotin and DOPE desbiotin fused cells. Desbiotin is expected to show less SAV binding as the affinity is about four orders of magnitude lower; however, that does not by itself explain that no significant binding is observed at all cells incubated with DOPE-desbiotin as the affinity of desbiotin for streptavidin is still very high $\left(10^{-11} \mathrm{M}\right){ }^{45}$. An additional cause might be the slightly shortened linker that connects desbiotin to DOPE, possibly sterically hindering proper binding of desbiotin to the SAV binding pocket. The clearly positive SAV-APC signal of MCF-7 cells after incubation with 100 mol\% desbiotin liposomes (Appendix 9.C, Panel E) indicates that the $20 \mathrm{~mol} \%$ desbiotin-DOPE is inadequate to evaluate fusion of the liposomes with cells whereas $100 \mathrm{~mol} \%$ is.

Importantly, the incorporated (des)biotin handles into the membrane of liposomes can have several applications apart from the evaluation of their fusion with cells. Pereira et al. reported that purification of docetaxel-loaded liposomes from free docetaxel drug using size exclusion chromatography resulted to 3.6-fold decrease in drug loading ${ }^{46}$; the presence of (des)biotin of our liposome systems in a similar drug application could serve as reversible anchors for their purification from the free drug molecules. More specifically, the use of desbiotin as well as chol-EG -biotin biotin provides the possibility of reversibility via the capture of drug-loaded liposomes using streptavidin (SAV-columns or SAV-ferrofluid) and release, by using $\beta$-cyclodextrin ${ }^{47}$ or excess free biotin ${ }^{48}$ respectively. Following the same approach, targeted isolation of cells having fused with the drug-loaded liposomes could be potentially isolated and analyzed to evaluate the effect of drug administration.

Last but not least, the chol-EG -biotin liposomes could have an important application in the extracellular vesicle field. Extracellular vesicles-derived from mesenchymal stem cells have been reported to play a cardio-protective role ${ }^{49}$ and have been suggested as therapeutic agents in liver ${ }^{50}$ and myocardial ${ }^{51}$ diseases. Their fusion with smaller size liposomes bearing desired receptors on their surface could increase their tropism to the target site. Furthermore, they could also be fused with liposomes loaded with a desired drug ${ }^{52,53}$ to take advantage of their further increased circulation lifetime and lower immunogenicity compared to the artificially formed liposomes or other synthetic drug carriers ${ }^{54}$. 


\section{References}

1. Lasic D. Recent developments in medical applications of liposomes: sterically stabilized liposomes in cancer therapy and gene delivery in vivo. J Controlled Release (Article|Proceedings Paper) 1997; 48(2-3): 203-222; doi 10.1016/S0168-3659(97)00045-X.

2. Gregoriadis G, Ryman BE. Liposomes as carriers of enzymes or drugs: a new approach to the treatment of storage diseases. Biochem J 1971; 124(5): 58P.

3. Matsumura Y, Kataoka K. Preclinical and clinical studies of anticancer agent-incorporating polymer micelles. Cancer Sci 2009; 100(4): 572-579.

4. Zamboni WC. Concept and clinical evaluation of carrier-mediated anticancer agents. Oncologist 2008; 13(3): 248-260; doi 10.1634/theoncologist.2007-0180.

5. Pratsinis A, Zuercher S, Forster V, Fischer EJ, Luciani P, Leroux JC. Liposomesupported enzymatic peritoneal dialysis. Biomaterials 2017; 145: 128-137; doi 10.1016/j. biomaterials.2017.08.016.

6. Etheridge ML, Campbell SA, Erdman AG, Haynes CL, Wolf SM, McCullough J. The big picture on nanomedicine: the state of investigational and approved nanomedicine products. Nanomedicine 2013; 9(1): 1-14; e-pub ahead of print 2012/06/06; doi 10.1016/j.nano.2012.05.013.

7. Sarkar D, Vemula PK, Zhao W, Gupta A, Karnik R, Karp JM. Engineered mesenchymal stem cells with self-assembled vesicles for systemic cell targeting. Biomaterials 2010; 31(19): 52665274; e-pub ahead of print 2010/04/08; doi 10.1016/j.biomaterials.2010.03.006.

8. Sarkar D, Vemula P, Teo G, Spelke D, Karnik R, Wee L et al. Chemical Engineering of Mesenchymal Stem Cells to Induce a Cell Rolling Response. Bioconjugate Chem (Article) 2008; 19(11): 2105-2109; doi 10.1021/bc800345q.

9. Dutta D, Pulsipher A, Luo W, Mak H, Yousaf MN. Engineering Cell Surfaces via Liposome Fusion. Bioconjugate Chem 2011; 22(12): 2423-2433; doi 10.1021/bc200236m.

10. Kawahara K, Sekiguchi A, Kiyoki E, Ueda T, Shimamura K, Kurosaki Y et al. Effect of TRXliposomes size on their prolonged circulation in rats. Chem Pharm Bull (Tokyo) 2003; 51(3): 336-338.

11. Noble GT, Stefanick JF, Ashley JD, Kiziltepe T, Bilgicer B. Ligand-targeted liposome design: challenges and fundamental considerations. Trends Biotechnol 2014; 32(1): 32-45.

12. Paszko E, Senge MO. Immunoliposomes. Curr Med Chem 2012; 19(31): 5239-5277.

13. Charoenphol P, Huang RB, Eniola-Adefeso O. Potential role of size and hemodynamics in the efficacy of vascular-targeted spherical drug carriers. Biomaterials 2010; 31(6): 1392-1402.

14. Poste G, Bucana C, Raz A, Bugelski P, Kirsh R, Fidler IJ. Analysis of the fate of systemically administered liposomes and implications for their use in drug delivery. Cancer research 1982; 42(4): 1412-1422.

15. Hunt CA, Rustum YM, Mayhew E, Papahadjopoulos D. Retention of cytosine arabinoside in mouse lung following intravenous administration in liposomes of different size. Drug Metab Dispos 1979; 7(3): 124-128.

16. Charoenphol P, Onyskiw PJ, Carrasco-Teja M, Eniola-Adefeso O. Particle-cell dynamics in human blood flow: implications for vascular-targeted drug delivery. J Biomech 2012; 45(16): 2822-2828; e-pub ahead of print 2012/09/23; doi 10.1016/j.jbiomech.2012.08.035. 
17. Namdee K, Thompson AJ, Charoenphol P, Eniola-Adefeso O. Margination propensity of vascular-targeted spheres from blood flow in a microfluidic model of human microvessels. Langmuir 2013; 29(8): 2530-2535; e-pub ahead of print 2013/02/08; doi 10.1021/la304746p.

18. Fang J, Nakamura H, Maeda H. The EPR effect: Unique features of tumor blood vessels for drug delivery, factors involved, and limitations and augmentation of the effect. Adv Drug Deliver Rev 2011; 63(3): 136-151.

19. Torchilin V. Tumor delivery of macromolecular drugs based on the EPR effect. Adv Drug Deliver Rev 2011; 63(3): 131-135.

20. Li S-D, Chen Y-C, Hackett MJ, Huang L. Tumor-targeted delivery of siRNA by self-assembled nanoparticles. Molecular therapy : the journal of the American Society of Gene Therapy 2008; 16(1): 163-169.

21. Kraft JC, Freeling JP, Wang Z, Ho RJY. Emerging research and clinical development trends of liposome and lipid nanoparticle drug delivery systems. Journal of pharmaceutical sciences 2014 103(1): 29-52.

22. Alshehri A, Grabowska A, Stolnik S. Pathways of cellular internalisation of liposomes delivered siRNA and effects on siRNA engagement with target mRNA and silencing in cancer cells. Sci Rep 2018; 8(1): 3748; doi 10.1038/s41598-018-22166-3.

23. Ziello JE, Huang Y, Jovin IS. Cellular endocytosis and gene delivery. Mol Med 2010; 16(5-6): 222-229; doi 10.2119/molmed.2009.00101.

24. Vasir JK, Labhasetwar V. Biodegradable nanoparticles for cytosolic delivery of therapeutics. Adv Drug Deliv Rev 2007; 59(8): 718-728; doi 10.1016/j.addr.2007.06.003.

25. Gilleron J, Querbes W, Zeigerer A, Borodovsky A, Marsico G, Schubert U et al. Imagebased analysis of lipid nanoparticle-mediated siRNA delivery, intracellular trafficking and endosomal escape. Nat Biotechnol 2013; 31(7): 638-646; doi 10.1038/nbt.2612.

26. Yang J, Bahreman A, Daudey G, Bussmann J, Olsthoorn RC, Kros A. Drug delivery via cell membrane fusion using lipopeptide modified liposomes. ACS central science 2016; 2(9): 621-630.

27. Frojmovic MM, Panjwani R. Geometry of normal mammalian platelets by quantitative microscopic studies. Biophys J 1976; 16(9): 1071-1089; e-pub ahead of print 1976/09/01; doi 10.1016/s0006-3495(76)85756-6.

28. Lee RG. leukocytes-the phagocytic and immunologic systems. In: Wintrobe's Clinical Haematology, Vol 1, 9th edn. Lippincott Williams \& Wilkins1983.

29. de la Rica R, Aili D, Stevens MM. Enzyme-responsive nanoparticles for drug release and diagnostics. Adv Drug Deliver Rev 2012; 64(11): 967-978.

30. Besse HC, Bos C, Zandvliet MMJM, van der Wurff-Jacobs K, Moonen CTW, Deckers R. Triggered radiosensitizer delivery using thermosensitive liposomes and hyperthermia improves efficacy of radiotherapy: An in vitro proof of concept study. Plos One 2018; 13(9): e0204063.

31. Needham D, Anyarambhatla G, Kong G, Dewhirst MW. A new temperature-sensitive liposome for use with mild hyperthermia: characterization and testing in a human tumor xenograft model. Cancer research 2000; 60(5): 1197-1201. 
32. Lee S-Y, Tyler JY, Kim S, Park K, Cheng J-X. FRET imaging reveals different cellular entry routes of self-assembled and disulfide bonded polymeric micelles. Molecular pharmaceutics 2013; 10(9): 3497-3506.

33. Braun T, Kleusch C, Naumovska E, Merkel R, Csiszar A. A bioanalytical assay to distinguish cellular uptake routes for liposomes. Cytometry Part A : the journal of the International Society for Analytical Cytology 2016; 89(3): 301-308.

34. Jung $\mathrm{H}$, Robison AD, Cremer PS. Multivalent ligand-receptor binding on supported lipid bilayers. J Struct Biol 2009; 168(1).

35. Kuhn SH, Gemperli B, Shephard EG, Joubert JR, Weidemann PA, Weissmann G et al. Interaction of liposomes with human leukocytes in whole blood. Biochim Biophys Acta 1983; 762(1): 119-127; doi 10.1016/0167-4889(83)90124-6.

36. Yang ST, Kreutzberger AJB, Lee J, Kiessling V, Tamm LK. The role of cholesterol in membrane fusion. Chem Phys Lipids 2016; 199: 136-143; doi 10.1016/j.chemphyslip.2016.05.003.

37. Churchward MA, Rogasevskaia T, Brandman DM, Khosravani H, Nava P, Atkinson JK et al. Specific lipids supply critical negative spontaneous curvature--an essential component of native Ca2+-triggered membrane fusion. Biophys J 2008; 94(10): 3976-3986; doi 10.1529/ biophysj.107.123984.

38. Chen Z, Rand RP. The influence of cholesterol on phospholipid membrane curvature and bending elasticity. Biophys J 1997; 73(1): 267-276; doi 10.1016/S0006-3495(97)78067-6.

39. Aeffner S, Reusch T, Weinhausen B, Salditt T. Energetics of stalk intermediates in membrane fusion are controlled by lipid composition. Proc Natl Acad Sci U S A 2012; 109(25): E1609-1618; doi 10.1073/pnas.1119442109.

40. Churchward MA, Rogasevskaia T, Hofgen J, Bau J, Coorssen JR. Cholesterol facilitates the native mechanism of Ca2+-triggered membrane fusion. J Cell Sci 2005; 118(Pt 20): 4833-4848; doi 10.1242/jcs.02601.

41. Lee DE, Lew MG, Woodbury DJ. Vesicle fusion to planar membranes is enhanced by cholesterol and low temperature. Chemistry and physics of lipids 2013; 166: 45-54.

42. Sun X, Whittaker GR. Role for influenza virus envelope cholesterol in virus entry and infection. J Virol 2003; 77(23): 12543-12551.

43. Krasnici S, Werner A, Eichhorn ME, Schmitt-Sody M, Pahernik SA, Sauer B et al. Effect of the surface charge of liposomes on their uptake by angiogenic tumor vessels. Int J Cancer 2003; 105(4): 561-567; e-pub ahead of print 2003/04/25; doi 10.1002/ijc.11108.

44. Miller CR, Bondurant B, McLean SD, McGovern KA, O’Brien DF. Liposome-cell interactions in vitro: Effect of liposome surface charge on the binding and endocytosis of conventional and sterically stabilized liposomes. Biochemistry-Us 1998; 37(37): 12875-12883; doi DOI 10.1021/ bi980096y.

45. Green NM. Avidin. In: Anfinsen CB, Edsall JT, Richards FM (eds.) Advances in Protein Chemistry, Vol 29 Academic Press1975, pp 85-133.

46. Pereira S, Egbu R, Jannati G, Al-Jamal WT. Docetaxel-loaded liposomes: The effect of lipid composition and purification on drug encapsulation and in vitro toxicity. International journal of pharmaceutics 2016; 514(1): 150-159. 
47. Beseničar MP, Bavdek A, Kladnik A, Maček P, Anderluh G. Kinetics of cholesterol extraction from lipid membranes by methyl- $\beta$-cyclodextrin-A surface plasmon resonance approach. Biochim Biophys Acta 2008; 1778(1): 175-184; doi 10.1016/j.bbamem.2007.09.022.

48. Hirsch JD, Eslamizar L, Filanoski BJ, Malekzadeh N, Haugland RP, Beechem JM et al. Easily reversible desthiobiotin binding to streptavidin, avidin, and other biotin-binding proteins: uses for protein labeling, detection, and isolation. Anal Biochem 2002;308(2):343-357; doi https://doi. org/10.1016/S0003-2697(02)00201-4.

49. Lai RC, Arslan F, Lee MM, Sze NSK, Choo A, Chen TS et al. Exosome secreted by MSC reduces myocardial ischemia/reperfusion injury. Stem Cell Res 2010; 4(3): 214-222.

50. Lou $G$, Chen Z, Zheng M, Liu Y. Mesenchymal stem cell-derived exosomes as a new therapeutic strategy for liver diseases. Exp Mol Med 2017; 49(6): e346.

51. Adamiak M, Sahoo S. Exosomes in Myocardial Repair: Advances and Challenges in the Development of Next-Generation Therapeutics. Molecular therapy : the journal of the American Society of Gene Therapy 2018; 26(7): 1635-1643.

52. Sun D, Zhuang X, Xiang X, Liu Y, Zhang S, Liu C et al. A novel nanoparticle drug delivery system: the anti-inflammatory activity of curcumin is enhanced when encapsulated in exosomes. Molecular therapy : the journal of the American Society of Gene Therapy 2010; 18(9): 1606-1614.

53. Haney MJ, Klyachko NL, Zhao Y, Gupta R, Plotnikova EG, He Z et al. Exosomes as drug delivery vehicles for Parkinson's disease therapy. Journal of controlled release : official journal of the Controlled Release Society 2015; 207: 18-30.

54. Murphy DE, de Jong OG, Brouwer M, Wood MJ, Lavieu G, Schiffelers RM et al. Extracellular vesicle-based therapeutics: natural versus engineered targeting and trafficking. Exp Mol Med 2019; 51(3): 32 . 


\section{Acknowledgments}

The authors acknowledge the Experimental Centre for Technical Medicine (ECTM) of the University of Twente for providing us with the blood samples of healthy individuals. 
A.

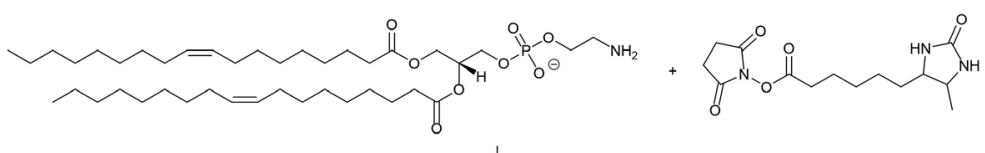

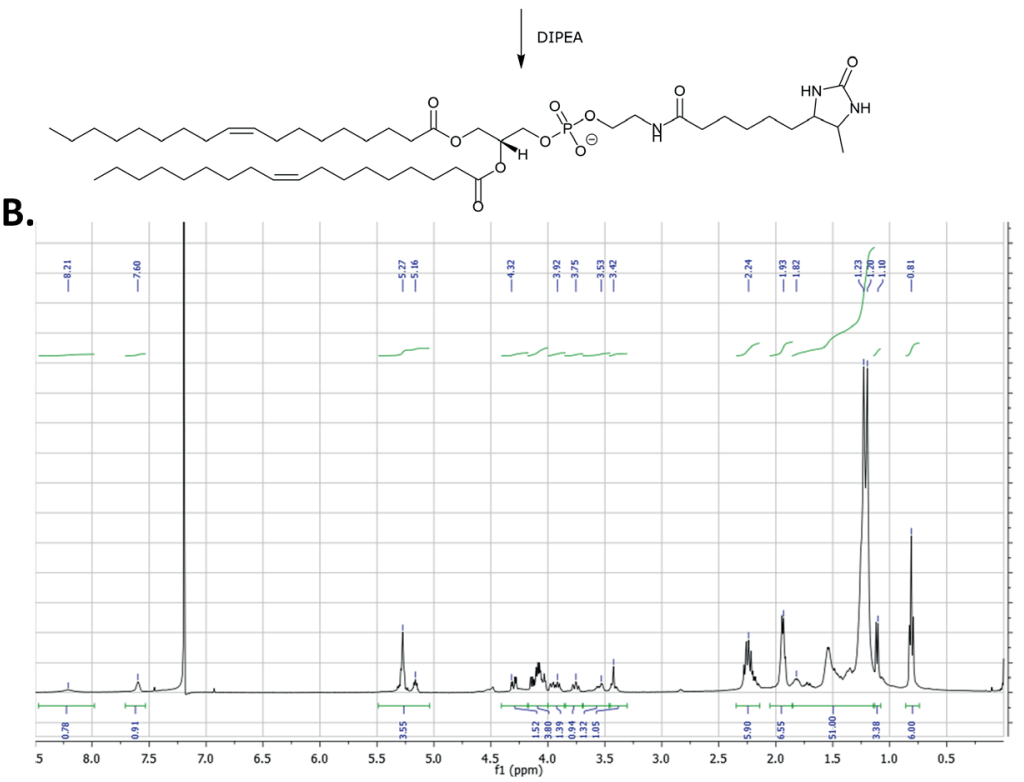

c.

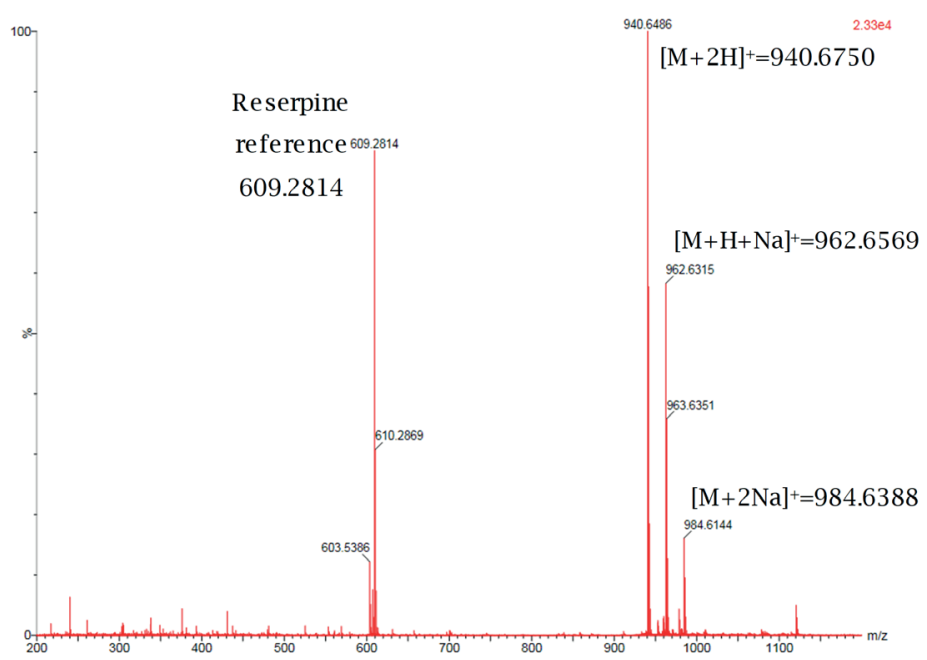

Appendix 9.A. Synthesis route of DOPE-desbiotin (Panel A). ${ }^{1} \mathrm{H}-\mathrm{NMR}$ of DOPE-desbiotin (Panel B). ESI(+)-ToF mass spectrum of DOPE-desbiotin. Reserpine was used as internal reference (Panel C). 


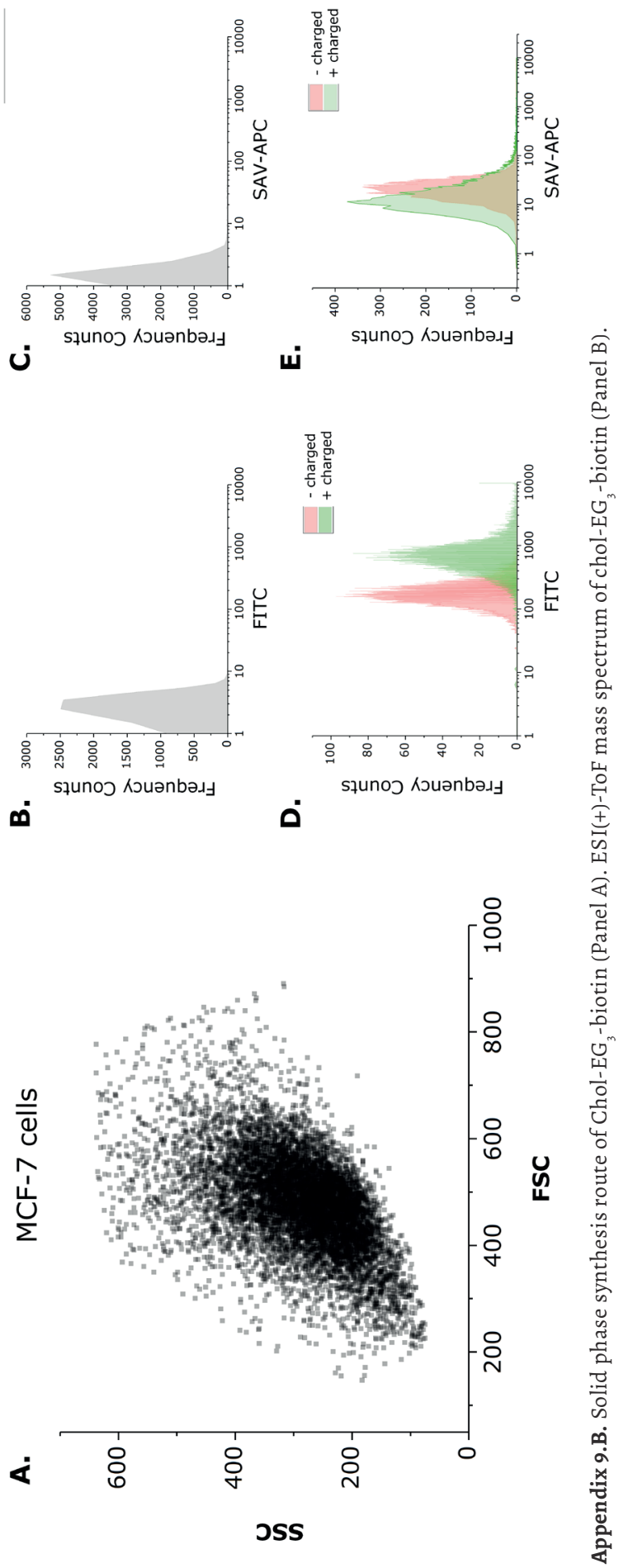



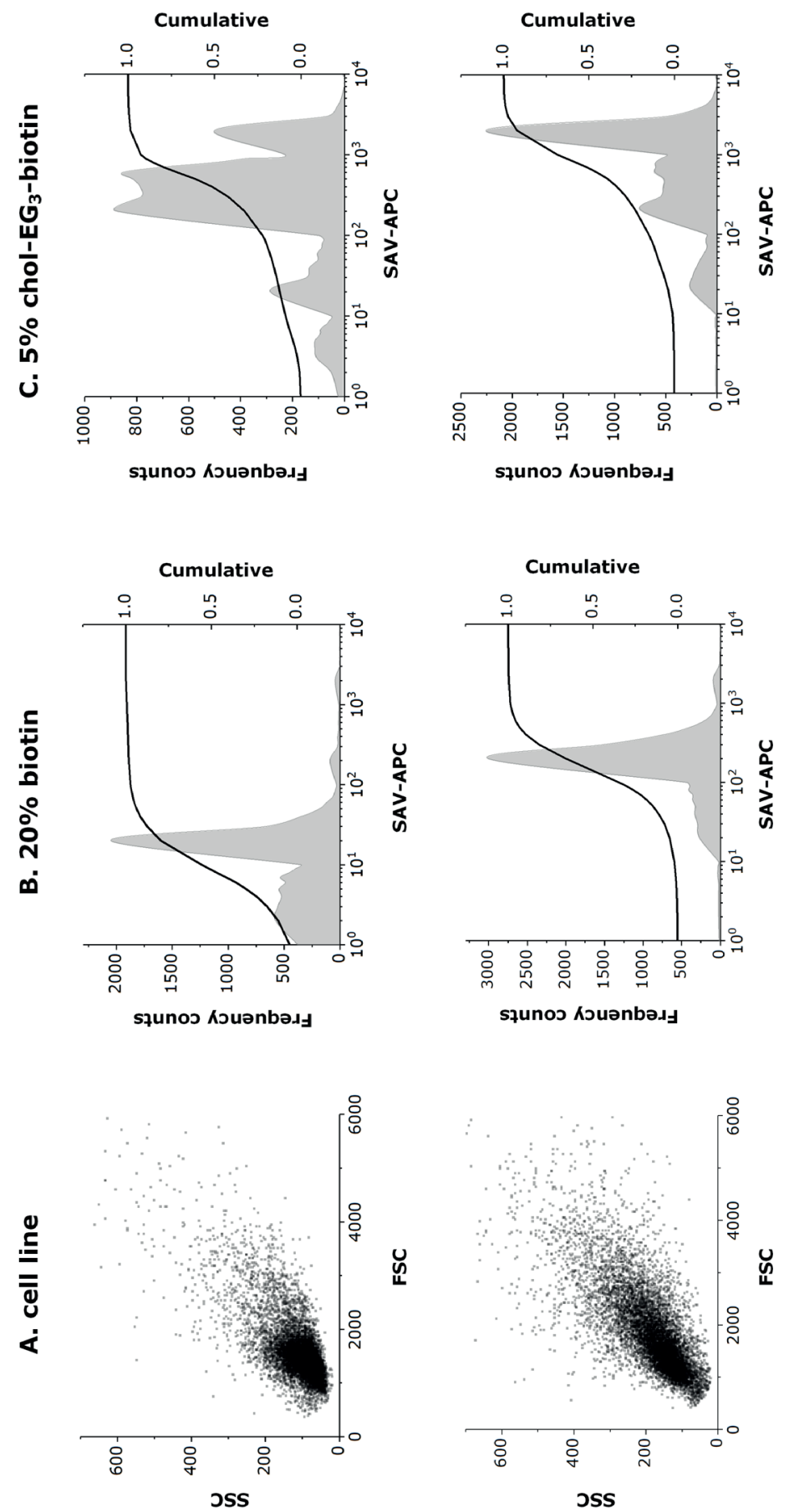

8S0ZV'I

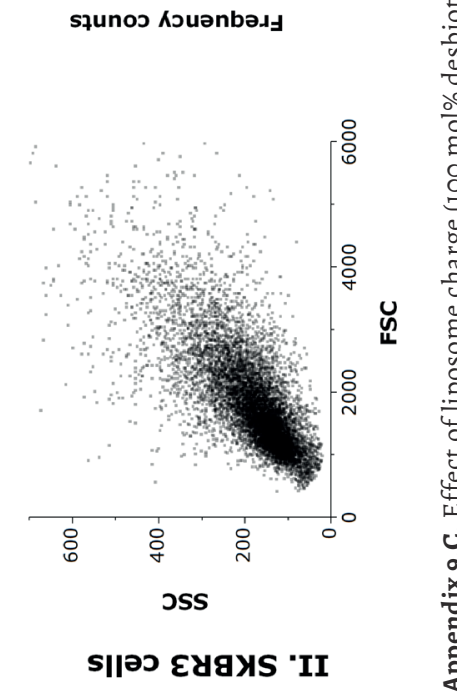


A.

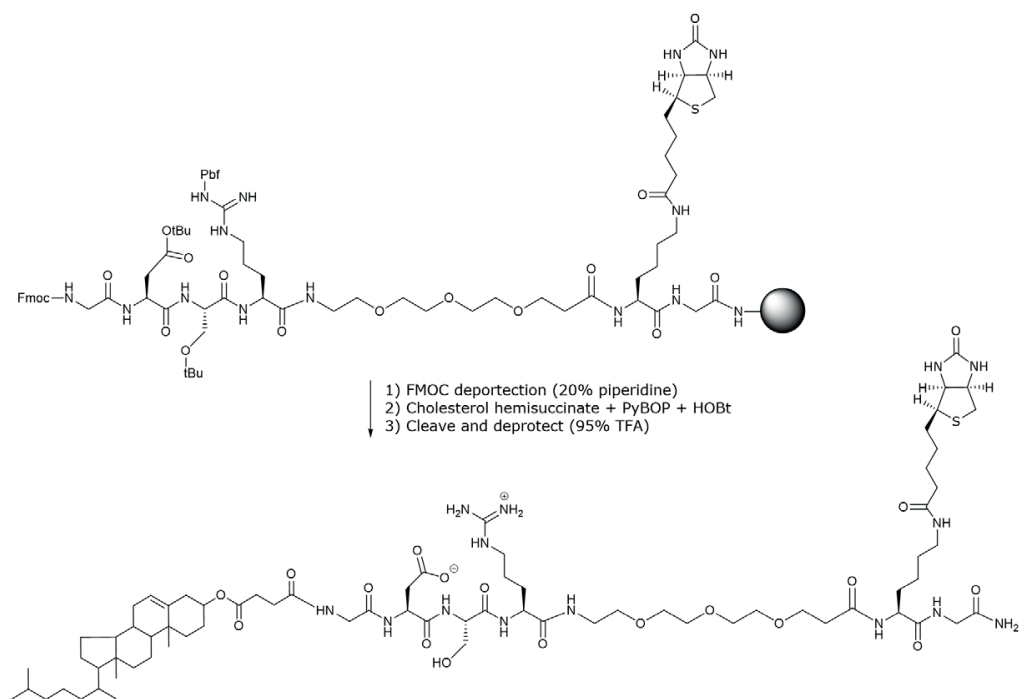

B.

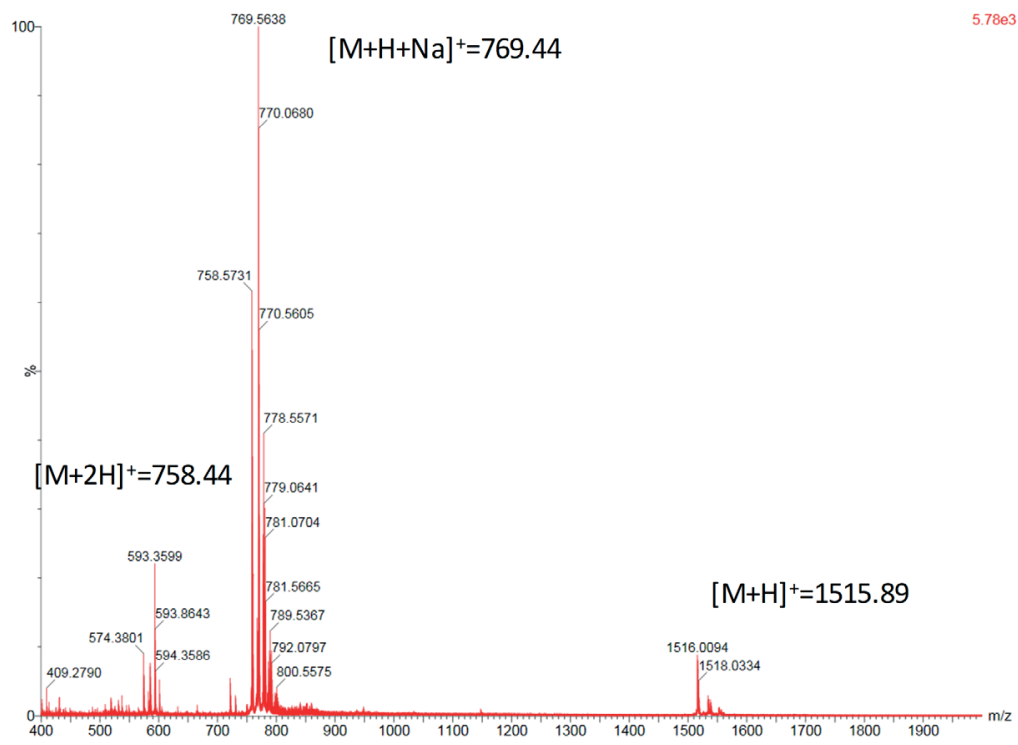

Appendix 9.D. Effect of cell size and surface morphology on fusion with liposomes 



\section{Chapter 10}

\section{Outlook}

Cancer patient care could ameliorate tremendously by liquid biopsies, leading to prolonged survival of patients, improved quality of life and limited side effects by administrated drugs. More specifically, liquid biopsies can

- detect the presence of minimal residual disease after surgical removal of a primary tumor with ostensibly unaffected lymph nodes,

- provide quantitative tools for a better reflection of the metastatic activity of the tumor,

- $\quad$ provide a more objective tool for the evaluation of treatment responses

- indicate the optimal treatment for the individual patient at any time during the disease.

When present in sufficient numbers, CTCs represent the ideal "liquid biopsy" as they contain proteins, RNAs and DNAs that characterize the tumor cells of the patient including the ones responsible for the metastasis. The large tdEVs, described in this thesis, are detected in frequencies higher by at least an order of magnitude as compared to CTCs. The observation that they are equally prognostic to CTCs implies a reflection of the metastatic activity/aggressiveness of the tumor; therefore, tdEVs can be very useful in the design of clinical trials for the evaluation of a new drug by properly balancing the two treatment arms (with patients of similar prognosis).

Suggestions to improve the isolation and identification of CTCs and tdEVs are provided below. 


\subsection{Increased sensitivity of CTC and tdEV detection 10.1.1 additional detection markers}

Large EpCAM+ tdEVs were identified using phycoerythrin-conjugated antibodies against the intracellular CK8, 18 and 19 (CK-PE) as a detection marker. However, scanning electron microscopy (SEM) images revealed unidentified CK-, CD45-, DAPI- particles (Chapter 5). The number of undetected particles becomes even higher when diagnostic leukapheresis (DLA) samples are processed with the CellSearch assay (Figure 10.1) suggesting the need to include more antibodies in the staining mixture to increase the number of CTCs and tdEVs after EpCAM enrichment. Lack or low expression of Cytokeratin in EpCAM+ CTCs and tdEVs is the most likely explanation. The inclusion of additional detection antibodies expressed on these EpCAM+ cells or the addition of EpCAM antibodies identifying different epitopes than the one used on the ferrofluids could address this issue.
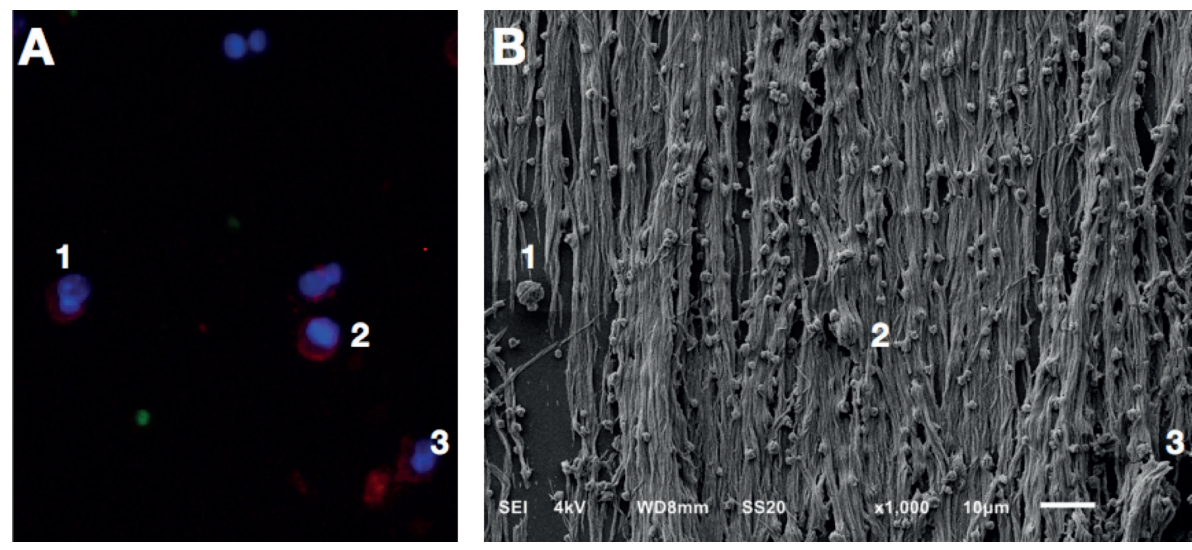

Figure 10.1. Correlated (A) fluorescence microscopy and (B) SEM image of EPCAM enriched cells from a diagnostic leukapheresis (DLA) sample of a castration-resistant prostate cancer patient. Green indicates $\mathrm{CK}$, red $\mathrm{CD} 45$ and blue nucleus.

The presence of $\mathrm{CK}-/ \mathrm{CK}_{\text {low }}$ particles is supported by our findings that HER2+, CK-, CD45-, DAPI- objects, isolated with the CellSearch assay, correlated strongly with the overall survival of metastatic breast cancer patients (Chapter 6). Moreover, staining of cancer cell lines with fluorescently labeled antibodies against EPCAM and CK (Figure 2) supports that most secreting (or secreted) vesicles are EpCAM+ but CK-.

Taking into consideration the extensive secretion of vesicles with a wide size range from a single cell as demonstrated by SEM (Figure 10.3), it becomes clear that the majority of EpCAM+ EVs will not be identified by the inclusion of solely fluorescent antibodies against CKs, since already a substantial portion of the large ones are not detectable by CK staining (Figure 10.2). 
Hoechst

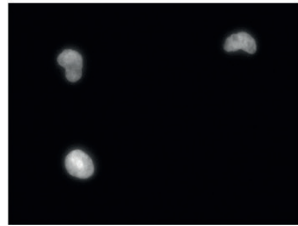

EpCAM-PE

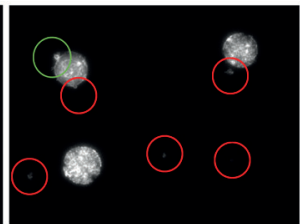

CK-APC

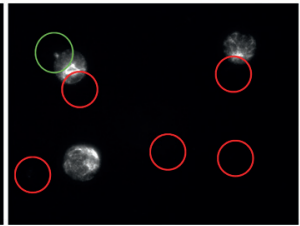

composite

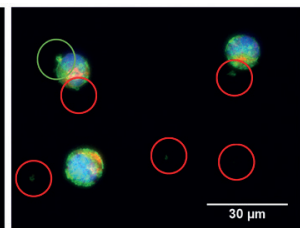

Figure 10.2. Immunofluorescence images of LNCaP prostate cancer cells, labeled with the nuclear dye Hoechst, phycoerythrin-conjugated antibody against EpCAM and allophycocyanin-conjugated antibody against CKs 8, 18 and 19. EpCAM+, CK- (extracellular) vesicles are enclosed within red circles, whereas the single EpCAM+, CK+ vesicle is enclosed within a green circle (acquisition of images by $\mathrm{N}$. Kamal).
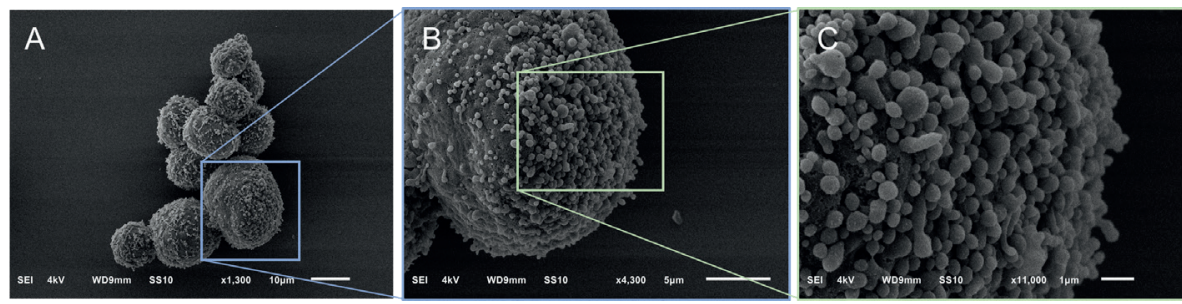

Figure 10.3. SEM image of $\mathrm{PC}_{3}$ cells in the act of secreting vesicles. (A) SEM image of an aggregate of $\mathrm{PC}_{3}$ cells, (B) SEM image of higher magnification of the $\mathrm{PC}_{3}$ cell enclosed by the blue square of $\mathrm{A}$, (C) SEM image of higher magnification of the $\mathrm{PC}_{3}$ cell membrane surface enclosed by the green square of $B$, showing substantial secretion of vesicles from the cell surface.

A. EpCAM enriched plasma of healthy individual
B. EpCAM enriched plasma of metastatic prostate cancer patient
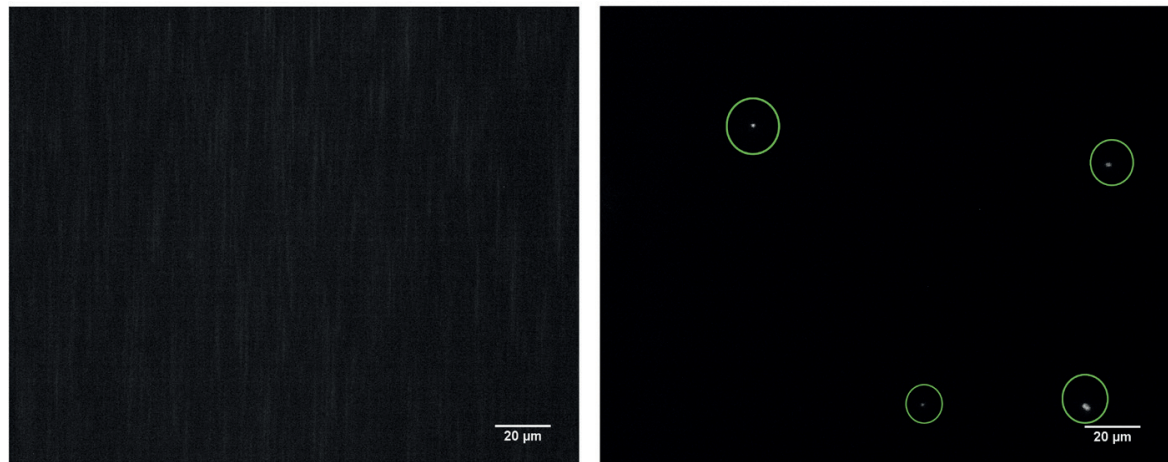

Figure 10.4. One out of 700 frames of PE channel, corresponding to CK signal, after EpCAM enrichment of $1 \mathrm{~mL}$ of (A) plasma of healthy individual and (B) plasma of metastatic prostate cancer patient using the CellSearch system. The images were acquired with a $40 x$ objective. If we extrapolate the counts of tdEVs for the 700 frames, around 5,600 CK+ events are expected to be found in $2 \mathrm{~mL}$ of metastatic cancer patient (corresponding to $7.5 \mathrm{~mL}$ of blood). The same patient had 65 CTCs and 1241 tdEVs when $7.5 \mathrm{~mL}$ of centrifuged blood was processed with the Cellsearch system. 


\subsection{2 label-free detection}

Detection and identification of tdEVs with label-free technologies such as Raman ${ }^{1,2,3}$, tested in the frames of the Cancer-ID Perspectief program, identified some alternative features of tdEVs that can be further explored.

\subsection{Increasing efficiency of tdEV isolation}

\subsection{1 analysis of the plasma fraction}

Based on model cancer cell lines, less than $1 \%$ of the totally secreted EVs will have a size larger than $2 \mu \mathrm{m}$ and as such, the majority of tdEVs will end up in the plasma fraction rather than the blood cell fraction. The number of isolated tdEVs can therefore increase around 100 times by running plasma samples of cancer patients with the CellSearch system or a similar EpCAM-based (immunomagnetic) enrichment.

Preliminary results (Figure 10.4) support that indeed CK+, DAPI-, CD45-tdEVs can be detected in plasma of cancer patients after positive selection based on EpCAM using the CellSearch system. Processing plasma of healthy donors does not result in similar detection of EpCAM+, CK+, CD45- events. The processing of plasma is of utmost importance, since it contains the majority of EVs and needs to be further investigated. An alternative approach tested within the frames of the Cancer-ID project, was the electrochemical detection and enumeration of EpCAM+ tdEVs, which allows easy quantification of tdEVs and relatively high throughput ${ }^{4}$. However, its performance remains to be demonstrated using blood samples from cancer patients.

\subsection{2 targeting other markers present on the tdEVs but not on other blood components}

Not all tdEVs shed by carcinomas are expected to express EpCAM molecules on their surface and in amounts sufficient for their capture by anti-EpCAM ferrofluids. Some tdEVs will have very few molecules simply because of their small size and surface available or due to lower EpCAM expression of their cells of origin.

Use of ferrofluids functionalized with multiple antibodies/proteins recognizing tumor-specific antigens, such as the heat shock protein $\mathrm{HsP70} 5,6$, the oncofetal chondroitin sulfate recognized by the VAR2CSA malaria protein ${ }^{7,8}$ or tissue-specific membrane markers, such as the prostate-specific membrane antigen (PSMA) for prostate and mammoglobulin for breast cancer could increase the capture efficacy and may identify different subclasses of tdEVs. 


\subsection{Clinical utility/added value of tdEVs}

The presence of tdEVs in higher frequencies could aid in the characterization of cancer and the treatment monitoring of patients in cases, where insufficient CTCs are detected.

The findings of Chapter 6 support that the phenotype of the human epidermal growth factor receptor 2 (HER2) of the resected tumor (as defined by fluorescence in situ hybridization, FISH) may be better reflected by tdEVs as compared to CTCs. Whether patients will have more clinical benefit or not from a treatment based on the original tissue, CTCs or tdEVs has yet to be addressed in prospective clinical trials. Probing for the presence of additional (membrane) therapeutic targets, such as the androgen, estrogen, progesterone, epidermal growth factor, G-protein coupled receptors or immune checkpoints, such as programmed death- ligand 1 (PD-Li) can further increase the potential clinical utility of CTCs and tdEVs.

Apart from the phenotypic characterization of tdEVs, further investigation on the encapsulated DNAs and RNAs within the large tdEVs is needed. In our definition used for the large tdEVs, there was no positive signal for DAPI staining, which makes it unlikely that sufficient nucleic acids are present to provide molecular information. The tumor microparticles identified by manual review in the original study ${ }^{9}$ included subclasses of tdEVs with and without nucleic acid staining. To evaluate whether useful molecular information can be extracted from tdEVs, all different tdEV subpopulations (as defined by manual scoring 9 as well as deep learning classification ${ }^{10}$ ) isolated from the blood cell fraction should be sorted -in a single or bulk level- and their nucleic acids quantified, amplified and analyzed for the detection of specific RNA or DNA aberrations. As suggested in the literature there are subclasses of tdEVs (exosomes, oncosomes) that are directly involved in the communication of tumor cells with their environment and may pave the environment for CTCs to nest and form metastases ${ }^{11-14}$. Whether or not the most clinically relevant tdEVs are present in the plasma fraction or the blood cell fraction is a question which cannot yet be answered. Towards that direction, sorting platforms of tdEVs should be established as the existing ones (VyCAP Puncher, Menarini DEPArray, ALS cellselector) have been developed for the sorting of only single cells.

A promising technology that has been used for the identification of point mutations and mRNAs in CTCs and that can be easily applied in the clinics is the in-situ padlock probe technology. The technology has been applied successfully in several CTC isolation technologies, including the CellSearch system, demonstrating positivity of AR-v7 mRNAs in CK+ and CK- (and otherwise undetectable) EpCAM+CTCs s, $16^{16}$ and detection of point mutations, such as KRAS in pancreatic cancer patients ${ }^{16}$. The applicability of such a technology on tdEVs has yet to be demonstrated and show whereas patients that could not be screened for the aforementioned treatment selection markers by CTCs can be "readable" by tdEVs. 


\subsection{Towards a more holistic approach of understanding cancer}

Each living organism constitutes a natural miracle already from the onset of its formation, but also later on, in terms of its development and sustained functionality. From the early steps of embryogenesis, a single stem cell has the potential to lead eventually to a fully independent organism via tightly controlled cell divisions and differentiation. The vitality of the organism is dependent on the health of each organ/tissue as well as the balance of all subunits. A diversity of cell types with totally different functions contribute in the proper functionality of each tissue and eventually the development or/and maintenance of the organism.

Likewise, tumor can be considered like a tissue, the growth and malignancy of which is not dependent solely on tumor cells but instead is tightly and dynamically interconnected with the tumor microenvironment. Tumor cells are stimulated by signals generated from a diversity of cell types, including endothelial cells, fibroblasts, inflammatory and dendritic cells. Via a network of signaling pathways, tumor malignancy and progression is promoted and regulated in a plethora of ways, described as hallmarks of cancer.

Therefore, it becomes clear that one-dimensional approach to understand cancer, eg the study of solely circulating tumor material or just imaging techniques to evaluate the size of the tissue and the extent of inflammation, cannot lead to the complete comprehension of disease onset and progression/remission. Whatever is not fully understood cannot be solved and in case of cancer, eradicated.

Quantitative assessment of markers in the clinics can ensure a more objective evaluation of the disease. At the same time, screening of a panel of markers will lead to a more holistic approach that is needed in the clinics for the better understanding of pathophysiological conditions. Extracellular vesicles hold a great promise as they serve as messengers of all eukaryotic cells, sending signals among them depending on the needs of each site. Therefore, eavesdropping the messages that have been sent from all contributing sites could identify the key factors pulling the strings of the disease. Signals from detected EVs can elaborate on the cell populations that act synergistically on disease progression by hyperactivity or abnormal silencing and pave the way for the direct or indirect eradication of diseases by the development of novel therapeutics. For instance, targeting the inflammation of the tumor instead the tumor burden itself has been proven to treat glioblastoma cases in animal models ${ }^{17}$. Furthermore, longitudinal measurements of EVs of different origins can shed light on EV dynamics over the course of treatments and help us comprehend underlying mechanisms of disease onset, progression and drug resistance. 


\section{References}

1. Beekman P, Enciso-Martinez A, Rho HS, Pujari SP, Lenferink A, Zuilhof $\mathrm{H}$ et al. Immunocapture of extracellular vesicles for individual multi-modal characterization using AFM, SEM and Raman spectroscopy. Lab Chip 2019; 19(15): 2526-2536; doi 10.1039/c9lco0081j.

2. Enciso-Martinez A, van der Pol E, Lenferink AT, Terstappen LW, van Leeuwen TG, Otto C. Synchronized Rayleigh and Raman scattering for the characterization of single optically trapped extracellular vesicles. Nanomedicine: nanotechnology, biology and medicine 2020; 24: 102109.

3. Lee W, Nanou A, Rikkert L, Coumans FAW, Otto C, Terstappen LWMM et al. Label-Free Prostate Cancer Detection by Characterization of Extracellular Vesicles Using Raman Spectroscopy. Anal Chem 2018; 90(19): 11290-11296; doi 10.1021/acs.analchem.8bo1831.

4. Mathew DG, Beekman P, Lemay SG, Zuilhof H, Le Gac S, van der Wiel WG. Electrochemical detection of tumor-derived extracellular vesicles on nanointerdigitated electrodes. Nano letters 2019.

5. Sherman MY, Gabai VL. Hsp70 in cancer: back to the future. Oncogene 2015; 34(32): 4153-4161; e-pub ahead of print 2014/10/28; doi 10.1038/onc.2014.349.

6. Boudesco C, Cause S, Jego G, Garrido C. Hsp70: A Cancer Target Inside and Outside the Cell. Methods Mol Biol 2018; 1709: 371-396; e-pub ahead of print 2017/11/28; doi 10.1007/978-1-49397477-1_27.

7. Agerbaek MO, Bang-Christensen SR, Yang MH, Clausen TM, Pereira MA, Sharma S et al. The VAR2CSA malaria protein efficiently retrieves circulating tumor cells in an EPCAMindependent manner. Nat Commun 2018; 9(1):3279; doi 10.1038/s41467-018-05793-2.

8. Agerbæk M $\varnothing$, Bang-Christensen SR, Yang M-H, Clausen TM, Pereira MA, Sharma S et al. The VAR2CSA malaria protein efficiently retrieves circulating tumor cells in an EpCAMindependent manner. Nature Communications 2018; 9(1): 3279; doi 10.1038/s41467-018-05793-2.

9. Coumans FAW, Doggen CJM, Attard G, de Bono JS, Terstappen LWMM. All circulating EpCAM+CK+CD45-objects predict overall survival in castration-resistant prostate cancer. Ann Oncol 2010; 21(9): 1851-1857; doi 10.1093/annonc/mdq030.

10. Zeune LLB, Y.; van Dalum, G.; Nanou, A.; de Wit, S.; Andree, K.; Swennenhuis, S.; van Gils , S.; Terstappen, L.W.M.M.; Brune, C. Deep learning of Circulating Tumour Cells. Nature Machine Intelligence (Research Article) 2020.

11. Hoshino A, Costa-Silva B, Shen T-L, Rodrigues G, Hashimoto A, Mark MT et al. Tumour exosome integrins determine organotropic metastasis. Nature 2015; 527(7578): 329-335.

12. Zhang H-G, Grizzle WE. Exosomes and cancer: a newly described pathway of immune suppression. Clinical Cancer Research 2011; 17(5): 959-964.

13. Costa-Silva B, Aiello NM, Ocean AJ, Singh S, Zhang H, Thakur BK et al. Pancreatic cancer exosomes initiate pre-metastatic niche formation in the liver. Nature cell biology 2015; 17(6): 816-826.

14. Minciacchi VR, You S, Spinelli C, Morley S, Zandian M, Aspuria PJ et al. Large oncosomes contain distinct protein cargo and represent a separate functional class of tumor-derived extracellular vesicles. Oncotarget 2015; 6(13): 11327-11341; doi 10.18632/oncotarget.3598. 
15. El-Heliebi A, Kashofer K, Fuchs J, Jahn SW, Viertler C, Matak A et al. Visualization of tumor heterogeneity by in situ padlock probe technology in colorectal cancer. Histochem Cell Biol 2017; 148(2): 105-115; doi 10.1007/s00418-017-1557-5.

16. El-Heliebi A, Hille C, Laxman N, Svedlund J, Haudum C, Ercan E et al. In Situ Detection and Quantification of AR-V7, AR-FL, PSA, and KRAS Point Mutations in Circulating Tumor Cells. Clin Chem 2018; 64(3): 536-546; doi 10.1373/clinchem.2017.281295.

17. Calle D, Negri V, Ballesteros P, Cerdan S. Magnetoliposomes loaded with poly-unsaturated fatty acids as novel theranostic anti-inflammatory formulations. Theranostics 2015; 5(5): 489503; doi 10.7150/thno.10069. 


\section{Summary}

After a general introduction in Chapter 1, we showed in Chapters $\mathbf{2}$ and 3, that large tdEVs in blood of metastatic prostate, breast, colorectal and non-small cell lung cancer patients have equivalent prognostic power as CTCs. Importantly, patients with metastatic prostate, breast and colorectal cancer with favorable CTC counts could be further stratified using tdEV counts implying that a subset of patients with favorable CTCs and unfavorable tdEV have a relatively poor outcome and may benefit from a more aggressive treatment similarly to the patients with unfavorable CTC counts.

In Chapter 4, we identified large leukocyte-derived extracellular vesicles (ldEVs) in blood of healthy individuals and metastatic cancer patients after immunomagnetic EpCAM enrichment and fluorescent labelling with the CellSearch system. Whereas tdEVs were 20-fold more frequent as compared to CTCs in metastatic cancer patients, the frequency of ldEVs were 5 -fold less frequent as compared to leukocytes coming along with the enrichment in both patients and controls. Fluorescence microscopy imaging of whole blood showed the presence of ldEVs in a 3-fold lower frequency as compared to leukocytes suggesting that the "fragmentation" of leukocytes into ldEVs is not caused by processing of blood samples in the CellSearch system and thus, are actually present in blood.

In Chapter 5, protocols were developed to image CTCs and tdEVs of castrationresistant prostate cancer patients isolated by the CellSearch system and CTCs isolated using $5 \mu \mathrm{m}$ filters by scanning electron microscopy (SEM). SEM images of CellSearch enriched CTCs and tdEVs were obtained, but detailed morphologic information was obscured by the presence of ferrofluid, whereas in case of filtration the cells were clearly deformed by the pressure the cells undergo, while entering the filter holes. Interestingly, using SEM many microparticles were observed with similar morphology and size as large tdEVs (EpCAM+, CK+, CD45-, DAPI-) but not detected by fluorescence microscopy. Whether they originate from the tumor or not remains to be further investigated.

In Chapter 6, the addition of the HER2 antibody in the CellSearch assay revealed the presence of HER2+, CK-, CD45-CTCs and tdEVs in the blood of breast cancer patients and had a similar association with poor clinical outcome as the CK+, CD45-CTCs. The larger frequency of tdEVs allowed the assessment of the presence of HER2 in a larger portion of patients and encourage the examination of more treatment targets on tdEVs. Importantly, these results pave the path towards a more rational and objective choice of patients who will or will not be subjected to HER2 targeting therapies. 
In Chapter 7, we compared the presence of CTCs and tdEVs before initiation of therapy and after the first cycle of therapy in CRPC, $\mathrm{mBC}$ and $\mathrm{mCRC}$ patients to evaluate the effect of therapy on CTCs versus tdEVs. The association between CTCs and tdEVs with overall survival was similar before the initiation of therapy but after the first cycle of therapy, CTCs outperformed tdEVs in mCRC implying that tdEV secretion is dependent on the treatment and possibly the cancer type. The distinction of different tdEV classes using a deep learning approach encourages us to determine the ones that rise or decline after the administration of an effective therapy in order to improve the evaluation of therapy responses and the patient treatment monitoring.

In Chapter 8, we investigated whereas we can detect endothelium-derived EVs (edEVs) in the CellSearch image datasets acquired from the CD146 enriched blood samples of metastatic colorectal cancer (mCRC) patients. Circulating endothelial cells (CECs) are significantly elevated in the blood circulation of cancer patients compared to healthy individuals; their presence is however not associated with better or poorer clinical outcome. The CEC number is biased by venipuncture procedure as endothelial cells are released due to the vacuum and enter the collection tube. edEVs should not be influenced; so we explored their presence through ACCEPT analysis and revealed that edEVs are detected at 5- to 10-fold higher frequencies compared to CECs. Moreover, their counts correlated with the clinical outcome of the patients. Importantly, the final multivariate Cox regression model included both tdEVs and edEVs as significant independent prognostic markers of the overall survival of $\mathrm{MCRC}$ patients. The elevated edEV counts denote either their active role in promoting tumor angiogenesis or/and their passive secretion because of the growth of the tumor. If the former hypothesis is correct, then edEVs could serve as a promising biomarker to predict patients that could benefit from anti-angiogenic treatments. Whether edEVs could serve as a more informative diagnostic tool in cardiovascular diseases remains to be investigated.

In Chapter 9, three different (des)biotin liposomes were compared in terms of their fusion with cells from cancer cell lines and blood from healthy individuals. DOPC liposomes of similar size distribution containing 20 mol\% DOPE-desbiotin, 20 mol\% DOPE-biotin or $5 \mathrm{~mol} \%$ chol-EG -biotin were prepared by extrusion. Their mean hydrodynamic diameter was around $100 \mathrm{~nm}$. Leukocyte subpopulations, platelets and different cancer cell lines were incubated with the different liposome systems, stained with fluorophore-tagged streptavidin and their fusion with liposomes was assessed by flow cytometry and immunofluorescence microscopy. The chol-EG -biotin liposomes achieved the highest biotin incorporation into the cell membrane for all different cell types, followed by DOPE-biotin liposomes. DOPE-desbiotin liposomes did not show considerable fusion with any cell type. The highest liposome uptake was found in cancer cells followed by the monocytes indicating a relationship between available cell surface and liposome uptake. 


\section{Samenvatting}

$\mathrm{Na}$ een algemene inleiding in Hoofdstuk $\mathbf{1}$ hebben we in Hoofdstuk $\mathbf{2}$ en $\mathbf{3}$ laten zien dat grote tdEVs in bloed van metastatische prostaat-, borst-, darm-, en niet-kleincellige longkankerpatiënten vergelijkbare prognostische waarde hebben als CTCs. Van belang is dat patiënten met metastatische prostaat-, borst- en darmkanker met gunstige CTC aantallen verder onderverdeeld konden worden door gebruik te maken van tdEV aantallen wat inpliceert dat een gedeelte van de patiënten met gunstige CTC aantallen en ongunstige tdEV aantallen een relatief slechte afloop hebben en voordeel kunnen hebben van een aggressievere behandeling evenals de patiënten met ongunstige CTC aantallen.

In Hoofdstuk $\mathbf{4}$ hebben we grote leukocyt-gederiveerde extracellulaire vesicles (ldEVs) geïdentificeerd in bloed van gezonde individuen en metastatische kankerpatiënten na immunomagnetische EpCAM verrijking en fluorescente kleuring met het Cellsearch systeem. Waar tdEVs 20 keer minder frequent waren in vergelijking met CTCs in metatstatische kankerpatiënten, was de frequentie van ldEVs vijf keer minder vergeleken met leukocyten die tijdens de verrijking meekwamen bij zowel patiënten als gezonde personen. Fluorescentie microscopie van bloed liet zien dat ldEVs in een drie keer lagere frequentie aanwezig waren vergeleken met leukocyten wat suggereert dat de "fragmentatie" van leukocyten in ldEVs niet veroorzaakt wordt door het verwerken van de bloedmonsters in het CellSearch systeem en dus aanwezig zijn in bloed.

In Hoofdstuk $\mathbf{5}$ hebben we protocollen ontwikkeld om CTCs en tdEVs van castratieresistente prostaatkankerpatiënten, geïsoleerd door het CellSearch systeem en CTCs geïsoleerd door gebruik te maken van $5 \mu \mathrm{m}$ filters, in beeld te brengen door scanning electron microscopy (SEM). SEM afbeeldingen van CellSearch verrijkte CTCs en tdEVs werden verkregen, maar gedetailleerde morfologische informatie werd vertroebeld door de aanwezigheid van ferrofluid (CellSearch), waar in het geval van filtratie duidelijk was dat de cellen vervormd waren door de druk die de cellen ondervonden tijdens het betreden van de filtergaten. Opmerkelijk was dat door het gebruik van SEM vele micropartikels geobserveerd zijn met vergelijkbare morfologie en grootte als grote tdEVs (EpCAM+, CK+, CD45-, DAPI-) maar dat deze niet gedetecteerd zijn met fluorescentie microscopie. Of deze voortkomen uit de tumor of niet moet nog verder onderzocht worden.

In Hoofdstuk 6 hebben we door toevoegen van het HER-2 antilichaam aan het CellSearchprotocol, ontdekt dat de aanwezigheid van HER2+, CK-, CD45- CTCs en tdEVs in bloed van borstkankerpatiënten vergelijkbare ongunstige klinische afloop heeft als CK+, CD45-CTCs. De grotere frequentie van tdEVs gaf de mogelijkheid om de HER-2 aanwezigheid in een groter deel van de patiënten te bepalen en motiveert verder onderzoek naar therapiemarkers op tdEVs. Van belang is dat deze resultaten een weg banen naar een meer rationale en objectieve keuze van patiënten die HER-2 gerichte therapie kunnen krijgen. 
In Hoofdstuk 7 hebben we de aanwezigheid van CTCs en tdEVs vóór initiatie van de behandeling en na de eerste cyclus van behandeling vergeleken in CRPC, mBC en mCRC patiënten om het effect van de behandeling op CTCs en tdEVs te evalueren. De relatie tussen CTCs en tdEVs met betrekking tot de uiteindelijke overlevingskans was vóór initiatie vergelijkbaar maar na de eerste cyclus overtroffen CTCs de tdEVs in mCRC wat impliceert dat tdEV secretie afhankelijk is van de behandeling en mogelijk het type kanker. Het onderscheid tussen verschillende tdEV klassen, geëvalueerd met een deep learning aanpak, motiveert ons om te onderzoeken of bepaalde klassen afof toenemen na het initiëren van een effectieve behandeling om op deze manier het monitoren van de behandeling te verbeteren.

In Hoofdstuk 8 onderzochten we of we endotheel-gederiveerde EVs (edEVs) konden detecteren in de CellSearch afbeeldingdatabase verkregen door de CD146 verrijkte bloedmonsters van metastatische darmkankerpatiënten (mCRC). Circulerende endotheelcellen (CECs) zijn significant verhoogd in de bloedcirculatie van kankerpatiënten vergeleken met gezonde personen maar de aanwezigheid van CECs is niet geassocieerd met een betere of slechtere klinische afloop. Het aantal CECs valt hoger uit vanwege het bloedprikken; tijdens de procedure komen endotheelcellen vrij die door het vacuüm in de buis gezogen worden. Het aantal edEVs zou niet beïnvloed mogen worden door het bloedprikken; deze aanwezigheid is bepaald door gebruik te maken van ACCEPT analyse en hierdoor werd duidelijk dat vijf tot tien keer meer edEVs gedetecteerd zijn dan CECs. Bovendien, de aantallen correleren met de klinische afloop van de patiënten. Van belang is dat het uiteindelijke multivariatie Cox regressiemodel beide tdEVs en edEVs includeert als significante onafhankelijke prognostische markers voor de uiteindelijke overlevingskans van MCRC patiënten. De verhoogde edEV aantallen duiden aan dat ze óf een actieve rol hebben in het bevorderen van de tumorangiogenese, óf een passieve secretie door de groei van de tumor. Als de eerste hypothese correct is dan zouden edEVs kunnen dienen als een veelbelovende biomarker om te voorspellen of patiënten voordeel kunnen hebben bij behandelingen die angiogenese tegengaan. Of edEVs kunnen dienen als een informatieve diagnostische tool bij cardiovasculaire aandoeningen moet nog verder worden onderzocht.

In Hoofdstuk 9 hebben we drie verschillende (des)biotineliposomen vergeleken inzake hun fusie met cellen bij kankercellijnen en bloed van gezonde personen. DOPC liposomen met vergelijkbare grootteverdeling met als inhoud 20 mol\% DOPE-

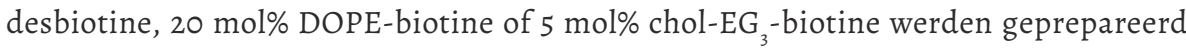
door extrusie. Hun gemiddelde hydrodynamische diameter was ongeveer $100 \mathrm{~nm}$. Leukocytsubpopulaties, bloedplaatjes en verschillende kankercellijnen werden geïncubeerd met de verschillende liposoomsystemen, gekleurd met fluorofoorgebonden streptavidine en hun fusie met liposomen werd bepaald door flowcytometrie en immunofluorescentie microscopie. De chol-EG -biotine liposomen bereikten de hoogste biotine-incorporatie in de celmembraan bij alle verschillende celtypen, 
gevolgd door de DOPE-biotine liposomen. DOPE-desbiotine liposomen lieten geen noemenswaardige fusie zien met enige cellijn. De hoogste liposoomopname werd gevonden in kankercellen gevolgd door monocyten wat wijst op een relatie tussen beschikbare celoppervlakte en liposoomopname. 



\section{List of publications}

\section{Journal Articles}

A. Nanou, F. Coumans, G. van Dalum, L.L. Zeune, D. Dolling, W. Onstenk, M. Crespo, M.S. Fontes, P. Rescigno, G. Fowler, P. Flohr, C. Brune, S. Sleijfer, J.S. de Bono, L.W.M.M Terstappen (2018), Circulating tumor cells, tumor-derived extracellular vesicles and plasma cytokeratins in castrationresistant prostate cancer, OncoTarget, 9 (27), 19283, DOI: 10.18632/oncotarget.25019

W. Lee, A. Nanou, L. Rikkert, F. Coumans, C. Otto, L.W.M.M. Terstappen, H. Offerhaus (2018), Labelfree Prostate Cancer Detection by Characterization of Extracellular Vesicles using Raman Spectroscopy, Analytical Chemistry, 90 (19), 11290-11296, DOI: 10.1021/acs.analchem.8bo1831

A. Enciso-Martinez", F. J. Timmermans", A. Nanou, L. W. M. M. Terstappen, C. Otto (2018), Towards high throughput SEM-Raman image cytometry of cells, Analyst, 143 (18), 4495-4502, DOI: 10.1039 / c8anoog55d

A. Nanou, P. Flohr, M. Crespo, J.S. de Bono, L.W.M.M. Terstappen (2018), Scanning electron microscopy of circulating tumor cells and tumor-derived extracellular vesicles, Cancers, 10 (11), 416, DOI:10.3390/ cancersio110416.

A. Nanou, L.L. Zeune, L.W.M.M. Terstappen (2019). Leukocyte-Derived Extracellular Vesicles in Blood with and without EpCAM Enrichment. Cells, 8 (8), 937, DOI: 10.3390/cells8080937

A. Nanou, M.C. Miller, L.L. Zeune, S. de Wit, C.J.A. Punt, H.J.M. Groen, D.F. Hayes, J.S. de Bono, L.W.M.M. Terstappen (2020), Tumour-derived extracellular vesicles in blood of metastatic cancer patients associate with overall survival, British Journal of Cancer, 122, 801-811, DOI: 10.108/ s41416-019-0726-9

L.L. Zeune, Y. Boink, G. van Dalum, A. Nanou, S. de Wit, K. Andree, J. Swennenhuis, S. van Gils, L.W.M.M. Terstappen, C. Brune (2020), Deep Learning of Circulating Tumour Cells, Nature Machine Intelligence, 2, 124-133, DOI: 10.1038/s42256-020-0153-x

L.G. Rikkert, P. Beekman, J. Caro, F.A.W. Coumans, A. Enciso Martinez, G. Jenster, S. Le Gac, W. Lee, T.G. van Leeuwen, G. B. Loozen, A. Nanou, R. Nieuwland, H.L. Offerhaus, C. Otto, D.M. Pegtel, M.C. Pionteek, E. van der Pol, L. de Rond, W.H. Roos, R.B.M. Schasfoort, M.H.M. Wauben, H. Zuilhof, L.W.M.M. Terstappen (2020), Cancer-ID: towards identification of cancer by tumor-derived extracellular vesicles in blood, Frontiers in oncology, DOI:10.3389/fonc.2020.00608

A. Nanou, L.L. Zeune, F.-C. Bidard, J.-Y. Pierga, L.W.M.M. Terstappen (2020), HER2 expression on tumor-derived extracellular vesicles and circulating tumor cells in metastatic breast cancer, submitted for publication

A. Nanou, L. Mol, M. Koopman, C.J.A. Punt, L.W.M.M. Terstappen (2020), Endothelium-derived extracellular vesicles associate with poor prognosis in metastatic colorectal cancer, submitted for publication 


\section{Conference contributions}

A. Nanou, C. Schwarzer, S. Le Gac, S. Schlatt, V. Nordhoff, M. Boiani, Applicability of a microfluidic device to produce blastocysts in vitro: how close is embryo quality in the device to quality in vivo, Selected poster presentation during the European Society of Human Reproduction and Embryology ESHR 2014, Munich, Germany, 29 June-2 July 2014

K. Andree, A. Mentink, S. de Wit, C. Brune, G. van Dalum, L.L. Zeune, A. Nanou, J. Swennenhuis, L.W.M.M. Terstappen, What is and what is not a Circulating Tumor Cell, Oral Presentation at the $10^{\text {th }}$ ISMRC International Symposium on Minimal Residual Cancer: Liquid Biopsy in Cancer Diagnostics and Treatment, Hamburg, Germany, 19-21 March 2016

A. Nanou, G. van Dalum, L. Zeune, F. Coumans, W. Onstenk, S. Sleijfer, M. Crespo, J. de Bono, L.W.M.M Terstappen, Circulating tumor cells, tumor extracellular vesicles and plasma cytokeratins in castration-resistant prostate cancer, Oral presentation at the 2016 Congress of the International Society for Extracellular Vesicles (ISEV2016), Rotterdam, the Netherlands, 4-7 May 2016

A. Enciso Martinez, A. Nanou, F. Timmermans, A. Lenferink, E. van der Pol, L.W.M.M. Terstappen, C. Otto: Identification and characterization of cells and extracellular vesicles (EVs) by SEM-Raman, Poster presentation at Dutch Biophysics 2016, Veldhoven, the Netherlands, 3-4 October 2016

A. Nanou, A. Enciso Martinez, P. Beekman, W. Lee, H. Suk Rho, S. Le Gac, C. van Rijn, H. Offerhaus, C. Otto, L.W.M.M. Terstappen, Cancer-ID: Isolation and characterization of tumor-derived extracellular vesicles, Poster presentation on the MIRA day 2016, Enschede, the Netherlands, 3 November 2016

W. Lee, A. Nanou, L.W.M.M. Terstappen, H.L. Offerhaus, Characterization of extracellular vesicles (EVs) using Raman spectroscopy, Poster presentation in Physics, Veldhoven, the Netherlands, 17-18 January 2017

A. Enciso Martinez, F. Timmermans, P. Beekman, A. Nanou, H. Rho, L.W.M.M. Terstappen and C. Otto, Correlative SEM-Raman for cell and extracellular vesicle characterization, Oral presentation in the Dutch Biophysics 2017, Veldhoven, the Netherlands, 2-3 October 2017.

L.L. Zeune, S. de Wit, G. van Dalum, K. Andree, J. Swennenhuis, A. Enciso Martinez, A. Nanou, A. Mentink-Leusink, L.W.M.M. Terstappen, L. Majunke, B. Zill, M. Alunni-Fabbroni, R. Lampignano, L. Yang, H. Neubauer, T. Fehm, M. Manicone, E. Rossi, R. Zamarchi, F. Farace, J.S. De Bono, C. Brune, Evaluating the consensus in circulating tumor cell scoring, Poster presentation in the $3^{\text {rd }}$ ACTC Advances in Circulating Tumor Cells Liquid Biopsy in Clinical Practice Congress (ACTC 2017), Rhodes, Greece, 4-7 October 2017.

A. Nanou, F. Coumans, G. van Dalum, L. Zeune, W. Onstenk, M. Crespo, M.S Fontes, P.Rescigno, G.Fowler, P. Flohr, C. Brune, S. Sleijfer, J. de Bono, L.W.M.M Terstappen, Circulating tumor cells, tumor-derived extracellular vesicles and plasma cytokeratins in castration-resistant prostate cancer, Oral presentation in the $3^{\text {rd }}$ ACTC Advances in Circulating Tumor Cells Liquid Biopsy in Clinical Practice Congress (ACTC 2017), Rhodes, Greece, 4-7 October 2017 
W. Lee, A. Nanou, L. Rikkert, F. Coumans, C. Otto, L.W.M.M. Terstappen, H. Offerhaus, Characterization of extracellular vesicles using Raman spectroscopy for label-free cancer detection, Poster presentation at the 2018 Congress of the International Society for Extracellular Vesicles (ISEV2018), Barcelona, Spain, 2-6 May 2018

A. Nanou, P. Flohr, M. Crespo, J.S. de Bono, L.W.M.M. Terstappen, Scanning electron microscopy of circulating tumor cells and tumor-derived extracellular vesicles, Poster presentation at the $11^{\text {th }}$ ISMRC International Symposium on Minimal Residual Cancer: Liquid Biopsy, Montpellier, France, 3-5 May 2018

L.L. Zeune, G. van Dalum, A. Nanou, S. de Wit, K. Andree, J. Swennenhuis, L.W.M.M. Terstappen, C. Brune, Deep learning to identify circulating tumor cells by ACCEPT, Poster presentation at the $11^{\text {th }}$ ISMRC International Symposium on Minimal Residual Cancer: Liquid Biopsy, Montpellier, France, 3-5 May 2018

A. Nanou, L.L. Zeune, S. de Wit, M.C. Miller, C.J. Punt, H.J.M. Groen, D.F. Hayes, J.S. de Bono, L.W.M.M. Terstappen, tumor-derived extracellular vesicles in blood of metastatic breast, colorectal, prostate and non-small cell lung cancer patients are associated with poor overall survival, Oral Presentation at the AACR Annual Meeting 2019, American Association for Cancer Research, Atlanta, USA, 29 March-3 April 2019

A. Nanou, L. Mol, M. Koopman, C.J.A. Punt, L.W.M.M. Terstappen, Tumor-derived and endotheliumderived extracellular vesicles in metastatic colorectal cancer patients, Poster presentation at the 4th ACTC meeting, Advances in Circulating Tumor Cells Liquid Biopsy in Clinical Practice Congress (ACTC 2019), Corfu, Greece, 2-5 October 2019

L.L. Zeune, Y.E. Boink, G. van Dalum, A. Nanou, S. de Wit, K.C. Andree, J.F. Swennenhuis, L.W.M.M Terstappen, C. Brune, Automated classification of circulating tumor cells by deep learning, Oral presentation at the 4th ACTC meeting, Advances in Circulating Tumor Cells Liquid Biopsy in Clinical Practice Congress (ACTC 2019), Corfu, Greece, 2-5 October 2019

A. Nanou, L. Mol, M. Koopman, C.J.A. Punt, L.W.M.M. Terstappen, Circulating tumor cells, tumorand endothelium-derived extracellular vesicles, but not circulating endothelial cells, associate with poor prognosis in colorectal cancer, Poster presentation at the AACR Annual meeting 2020, American Association for Cancer Research, San Diego, California, USA

A. Nanou, L.L. Zeune, F.-C. Bidard, J.-Y. Pierga, L.W.M.M. Terstappen, HER2 expression on tumor-derived extracellular vesicles and circulating tumor cells in metastatic breast cancer, Poster presentation at the AACR Annual meeting 2020, American Association for Cancer Research, San Diego, California, USA 



\section{Acknowledgments}

Welcome to the most difficult section of the book, which already starts with the bitterness that will remain incomplete since it is very difficult to include all people and circumstances that contributed -directly or indirectly-in the possibility I had to pursue a $\mathrm{PhD}$, the choice of the specific one as well as its completion. To begin with, I would like to aknowledge all people that played a decisive role on my arrival at Leon's department. Without you, I would most likely have a different (if any) book today: Lorenzo Moroni, Séverine Le Gac, Michele Boiani, Anouk Mentink-Leusink,

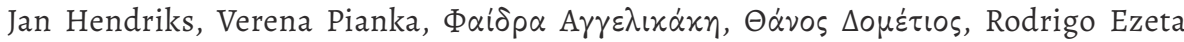
Aparicio and last but not least, my parents and my sister! Thanks to all of you, I made the decision to come back to the Netherlands and start a PhD with Leon!

Dear Leon, over these five years you kept setting higher and higher the standards I had for an ideal mentor. Thank you for being who you are. I will always admire your wonderfully simple way of thinking, your undiminished curiosity and interest on research, your attentiveness to your students' comments and ideas, your inhexaustible ideas, your fast feedback regardless the day or time of the week, your positivity and light and your constructively direct way. Our bi-weekly meetings were always an inspiration and delight to me. I am grateful I had the opportunity to pursue a PhD under your guidance. You will always occupy a special place to my life.

I am thankful to many bright colleagues, thoughtful scientistists, creative engineers and internationally recognized medical doctors who directly contributed to the completion of the present thesis. Thank you for sharing your excitement and ideas with me and all the insightful and constructive feedback and discussions! Special acknowledgements to Johann de Bono, Harry Groen, Pascal Jonkheijm, Guus van Dalum, Leonie Zeune, Craig Miller, Séverine Le Gac, Joost Swennenhuis, Frank Coumans and Sanne de Wit. Dear Mark (Verheijden), I would like to thank you for all the experiments you performed for the last liposome chapter, your beautiful ideas, enthousiasm, positive energy, eagerness and serenity regardless the overlap of our experiments with the last year of your PhD. I really enjoyed our collaboration! My dear chemistry guru, Dodo (Wasserberg), thank you for all the things you taught me and your suggestions when I was reaching a bottleneck!

Dear Leonie, thank you for developing the ACCEPT software, based on which $2 / 3$ of the present thesis was carried out. I am still wondering what if.. you had not left us! I am still recovering from the second stroke. So many plans and dreams that were left incomplete. I was always enjoying our -scientific or not-discussions and trips and I hope our roads will keep meeting in the one or the other way. Wish you all the best for you, Stefan and Oscar! 
Dear Anouk and Christian, thank you for your kindness and support -in all levelsanytime it was needed. Special acknowledgments for translating also the summary of my thesis! You are always having the promptness and willingness to provide us with solutions. The whole group relies on you and owes you a lot! The same stands for you, dear Ingrid: I was always impressed by the easiness and effectiveness you have to execute all actions that I was finding troublesome or/and annoying!

Dear -different over time- MCBP/VyCAP/BioEE/TLR members: Leon, Yoon, Hoon, Leonie, Andrea, Fikri, Peng, Pepijn, Guus, Kiki, Niels, Lisaki, Joost van D., Edwin, Joris, Michiel, Sanne, Agustin, Anouk, Aufried, Yvonne, Joost S., Xichen, Frank C., Frank T., Christian, Ingrid, Eshwari, Nargues, Richard, Armagan, Ruchi, Ana, Markus, Dodo, Cees, Joska, (all the people I forget or I do not know your names yet) and last but not least, my frienemy Arjan: thank you for all the moments you shared with me, all the trips, outings and activities (dinners, board games, weddings, dances, sessions of bouldering), the reflections, discussions and thoughts we shared, the amounts of caffeine, alcohol and sugar we consumed together! Thinking about them, makes me already nostalgic!

Dear Cancer-ID consortium: Pepijn, Leonie, Wooje, Hoon, Agustin, Linda, Melissa, Gyllion, Elmar and Edwin thank you for the creative activities (special credits to the organization skills of Linda and Agustin), trip to Mexico and the moments we spent together! I enjoyed all of them and wish you success and best of luck in your future decisions and carreers! Dear Pepijn, thank you for promoting our tdEV results in every possible occasion and all your kind and encouraging words or/and drinks!

I would like to thank all my officemates over these 5 years for all the discussions and insights on scientific (or other) topics as well as their patience and understanding for frustration peak hours (mainly at the last year) or social distancing (vacuum conditions while wearing my headphones): Joost S., Joost vD., Markus, Leonie, Michiel, Fikri, Peng, Ashwari and Frank (remotely). Thank you all for sharing your offices with me!

I should also address a big thank you to every single student I supervised during their internships/theses. Meike, Emeline, Xiang, Tanja, Dorothy, Nisat and Sjoerd, thank you for making me realize how satisfactory and refreshing can be to teach/explain when students are eager to learn. It is such a beautiful experience through which I also reflected and better understood myself (at least as a student).

Dear Belinda and Denisa, you could not be missing from my acknowledgments. Thank you for your positive, powerful energy and all your classes, where I kept releasing any stress or pressure I may had due to life circumstances during these last years. Because as our ancestors claimed: only a healthy body can sustain a healthy mind and vice versa

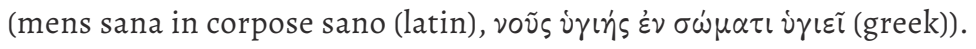


I am grateful to so many friends (outside the MCBP) for surrounding me, spending unforgettable (and - many times- embarrassing) moments with me, sharing similar craziness (not necessarily same interests), but most importantly, for reminding me my dreams when I seemed to forget them or feel discouraged about them: $\tau \rho \circ \mu \alpha \rho o v$ i $\alpha$,

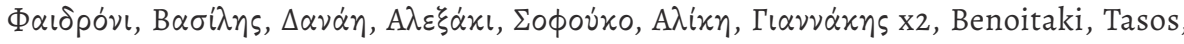

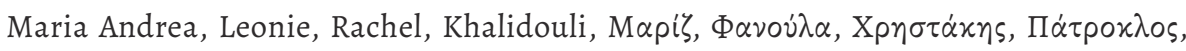

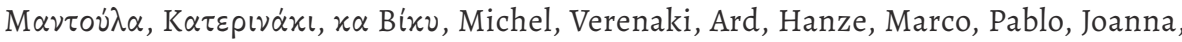

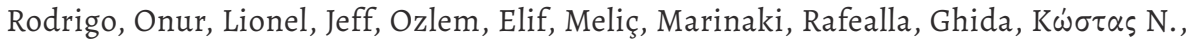

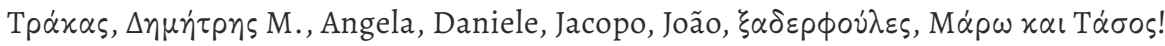

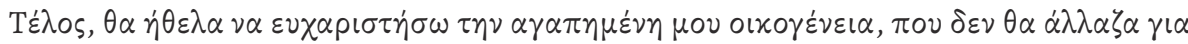

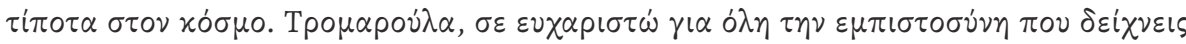

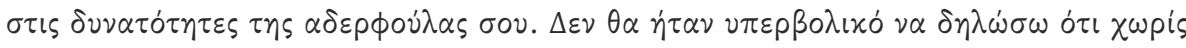

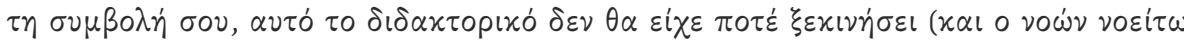

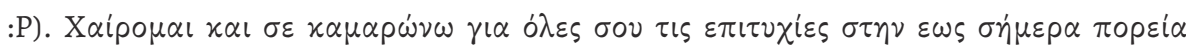

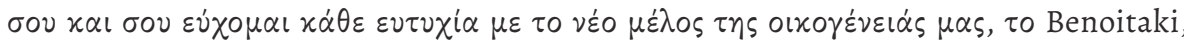

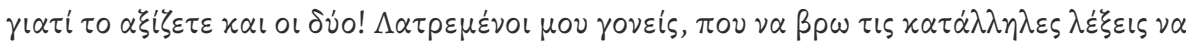

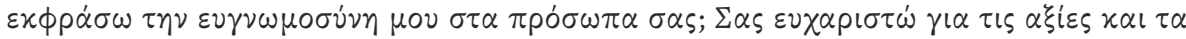

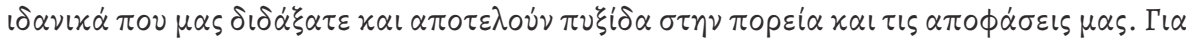

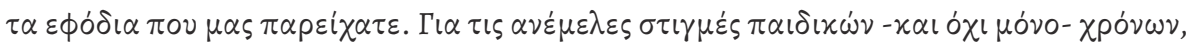

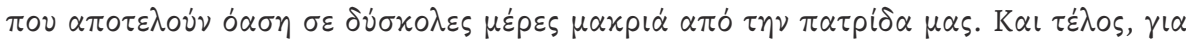

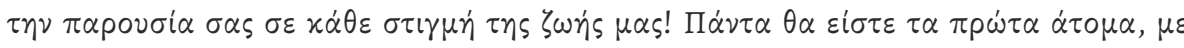

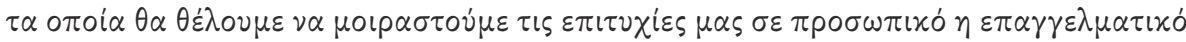

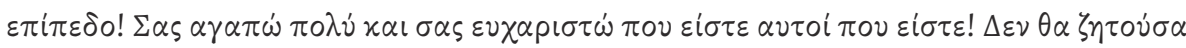
$\pi \circ \tau \varepsilon \dot{\delta} \delta\llcorner\phi \circ \rho \varepsilon \tau \iota x \alpha \dot{~} \pi \rho o ́ \tau v \pi \alpha$ !

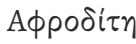





\section{Curriculum Vitae}

Afroditi Nanou, born in 1987 in Athens, Greece, graduated with honors from high school. In 2005, she gets a s scholarship and starts her studies in chemical engineering (specialization in food technology and biotechnology) at the National Technical University of Athens. At the last year of her studies, she conducts her diploma thesis, titled "Sugars acylation using esterases of acetic acid", at the Biotechnology and Environment lab (supervisors: P. Christakopoulos and E. Topakas). The thesis includes genetic modification of E.Coli, production and purification of esterases, optimization of the enzymatic acylation of mono- and poly- saccharides, thin-layer chromatography, purification of the acylated products and their identification by Fourier-transformed infrared spectroscopy and nuclear magnetic resonance.

After completing her MEng in 2010, she tries to find a job in her field, without much success. In the meantime, she works at the ADSL helpdesk of the Hellenic Telecommunications Organization, translates some chapters of engineering books (Fountas books) and teaches mathematics to primary school students.

In 2012, she applies and gets accepted to the University of Twente (UT) to follow a MSc program in biomedical engineering (specialization in molecular, cellular and tissue engineering). In September of 2013, she has the opportunity to carry out a 3 monthresearch internship at the Max Planck Institute (MPI) for Molecular Biomedicine (Mouse Embryology Laboratory, supervisor: Michele Boiani) in Muenster, Germany. The project is in collaboration with the UT (BIOS group, supervisor: S. Le Gac) and includes fabrication (UT), use and evaluation (MPI) of microfluidic devices for growing mouse embryos from zygote- to blastocyst-stage towards the improvement of assisted reproductive technologies. After the completion of her internship, she works at the Tissue Regeneration department (UT) on her master thesis, titled: "3D scaffolds: a tissue engineering approach to study breast cancer" using engineering as a tool to study and better understand the biology of breast cancer (supervisors: L. Moroni and I. Lorenzo Moldero).

The end of 2014 and onset of 2015 finds her in Greece, missing friends and studies in Enschede. The opportunity comes from the Medical Cell BioPhysics department of the UT and Leon Terstappen who offers her a $\mathrm{PhD}$ position in the Cancer-ID project, starting in May 2015. The rest can be found in the book you are holding in your hands. 